\title{
Annexin A5: pharmacology, radiopharmaceutical aspects, and cell death imaging
}

Citation for published version (APA):

Boersma, H. H. (2005). Annexin A5: pharmacology, radiopharmaceutical aspects, and cell death imaging. [Doctoral Thesis, Maastricht University]. Universiteit Maastricht. https://doi.org/10.26481/dis.20060406hb

Document status and date:

Published: 01/01/2005

DOI:

10.26481/dis.20060406hb

Document Version:

Publisher's PDF, also known as Version of record

\section{Please check the document version of this publication:}

- A submitted manuscript is the version of the article upon submission and before peer-review. There can be important differences between the submitted version and the official published version of record.

People interested in the research are advised to contact the author for the final version of the publication, or visit the DOI to the publisher's website.

- The final author version and the galley proof are versions of the publication after peer review.

- The final published version features the final layout of the paper including the volume, issue and page numbers.

Link to publication

\footnotetext{
General rights rights.

- You may freely distribute the URL identifying the publication in the public portal. please follow below link for the End User Agreement:

www.umlib.nl/taverne-license

Take down policy

If you believe that this document breaches copyright please contact us at:

repository@maastrichtuniversity.nl

providing details and we will investigate your claim.
}

Copyright and moral rights for the publications made accessible in the public portal are retained by the authors and/or other copyright owners and it is a condition of accessing publications that users recognise and abide by the legal requirements associated with these

- Users may download and print one copy of any publication from the public portal for the purpose of private study or research.

- You may not further distribute the material or use it for any profit-making activity or commercial gain

If the publication is distributed under the terms of Article $25 \mathrm{fa}$ of the Dutch Copyright Act, indicated by the "Taverne" license above, 
(C) H.H. Boersma, Sittard 2005

ISBN $90-5278-516-3$

ISBN $978-90-5278-516-5$

Engelstalige correctie: Anne Hilton

Vormgeving en omslagontwerp: Marleen B. Berg, www.mbberg.com

Foto auteur: Geertjan van Zonneveld/ Appie Derks, azM

Druk: Datawyse Boekproducties Maastricht 



\section{Annexin A5:}

Pharmacology, radiopharmaceutical aspects, and cell death imaging 



\section{Annexin A5:}

\section{Pharmacology, radiopharmaceutical aspects, and cell deatb imaging}

\section{Proefschrift}

Ter verkrijging van de graad van doctor aan de Universiteit Maastricht, op gezag van de Rector Magnificus, Prof, mr. G.P.M.F. Mols, volgens het besluit van het College van Decanen in het openbaar te verdedigen op donderdag 6 april 2006 om 14.00 uur

door

\section{Hendrikus Hessel Boersma}

geboren te Leeuwarden op 28 juni 1968 
? 


\section{Promotor:}

Prof. Dr. G.A.K. Heidendal

\section{Co-promotores:}

Dr. C.P.M. Reutelingsperger

Dr. L.M.L. Stolk

\section{Beoordelingscommissie:}

Prof. Dr. G.J.J. Teule (voorzitter)

Prof. Dr. C. Bruggeman

Prof. Dr. J. Narula (University of California, Irvine, Irvine, USA)

Dr. C. Neef

Prof. Dr. A. Verbruggen (Universiteit Leuven).

Het in dit proefschrift beschreven onderzoek is mogelijk gemaakt door steun van:

NWO

De Nederlandse Hartstichting

De stichting 'Onderzoek Klinische Farmacie'

De firma Hofsperger \& Co. 

Many who live, deserwe death, some who die, deserwe life.

Velen die leven, verdienen te sterven, sommigen die sterwen, verdienen te leven.

Gandalf, in: The Lord of the Rings, Part I. The Fellowsib of the Ring

To everything there is a season, and a time to every purpose under heaven. A time to be born, a time to die....

Ecclesiastes 3: 1-2a

(from the song Turn! Turn! Twrm! performed by The Byrds)

Alles heeft zijn uur, alle dingen onder de hemel hebben hun tijd.

Er is een tijd om geboren te worden en een tijd om te sterven....

Prediker $3: 1-2 \mathrm{a}$ 



\section{Contents}

1 General Introduction: Past, present and future of annexin A5:

from protein discovery to clinical applications

1.1 Introduction.

1.2 Past 21

1.2.1. Discovery

1.2.2 The structure of annexin A5 21

1.2.2.1 Primary structure and functional aspects

1.2.2.2 Terriary structure 23

1.2.3 Biophysical properties

1.2.4 Biochemical properties

1.2.5 The role of annexin A5 in physiology and disease

1.2.6 Development of annexin A5 as a diagnostic tool

1.3 Intermezzo: basic principles of cell death

1.4 Radiolabeled derivatives of annexin A5

1.4.1 Technetium-labeled annexin A5

1.4.1.1 ${ }^{99 m} \mathrm{~T} \mathrm{c}$ (n-1-imino-4-mercaptobutyl)-annexin A5 36

1.4.1.2 ${ }^{99_{m}}$ Tc-BTAP.annexin A5 36

1.4.1.3 ${ }^{99 \mathrm{~m}} \mathrm{Te}-\mathrm{EC}$-annexin AS 36

1.4.1.4 ${ }^{99 \mathrm{~m} T C-H Y N I C-a n n e x i n ~ A 5}$

1.4.1.5 Novel labeling methods using ${ }^{99 m}$ Tc-annexin A5 mutant proteins 37

1.4.2 Annexin A5 labelled with halogen radioisotopes

1.4.2.1 ${ }^{123} \mathrm{I} /{ }^{124} \mathrm{I} /{ }^{125} \mathrm{I}$-annexin A5

1.4.2.2 ${ }^{18} \mathrm{~F}$-labelled annexin $\mathrm{A} 5$

$1.4 .3^{11} \mathrm{In}-\mathrm{PEG}$-annexin A5

1.4.4 Conclusion

1.5 Quality control of radiolabeled annexin A5

1.6 Imaging applications for annexin A5 in animal studies 
1.6.3 Evaluation of cancer therapy $\quad 43$

1.6.4 Orher animal models $\quad 44$

1.7 Biodistribution and pharmacokinetics of radiolabeled annexin A5 in humans 46

1.7.1 Technetium Labeled annexin A5 46

$\begin{array}{ll}\text { 1.7.2 lodine labeled annexin } & 48\end{array}$

11.8 Current clinical nuclear medicine applications 49

1.8.1 Cardiovascular diseases $\quad 49$

1.8.1.1 Myocardial infarction $\quad 49$

1.8.1.2 Cardiac allograft rejection. $\quad 50$

1.8.1.3 Heart faidure $\quad 51$

1.8.1.4 Atherosclerosis 53

1.8.2 Cancer $\quad 54$

1.8.2.1 Intracardial tumors $\quad 55$

1.8.2.2 Head and neck cancer $\quad 59$

1.8.2.3 Other tumor types $\quad 59$

1.8.2.4 Cancer treatment efficacy evaluation using ${ }^{99 \mathrm{~m} T \mathrm{~T}-a n n e x i n}$ A5 $\quad 59$

1.9 Possible clinical pirfall: annexin A5 antibodies 61

1.10 Future $\quad 62$

1.11 Conclusions $\quad 63$

1.12 Objectives of this thesis $\quad 64$

2 The ApoCortect ${ }^{\text {TM }}$ assay: a novel, rapid method to derermine the biological functionality of tadiolabeled and Auorescent Annexin A5 69

$\begin{array}{ll}\text { 2.1 Introduction } & 70\end{array}$

2.2 Materials and Methods $\quad 71$

2.3 Results and Discussion. 75

2.4 Conclusion $\quad 84$

3 Comparison between human pharmacokinetics and imaging properties of two conjugation methods for ${ }^{9}$ Ta-Annexin A5 89

3,1 Introduction $\quad 90$

3.2 Materials and Methods 91

3.3 Results 95 
3.4 Discussion

3.5 Conclusion

4 Biodistribution and dosimetry of ${ }^{99}$ "To-BTAP-annexin A5 in humans

4.1 Introduction

4.2 Materials and Methods

4.3 Results

4.4 Discussion

4.5 Conclusion

5 Minocycline inhibits cell death in vivo in myocardial ischemia and subsequent reperfusion

5.1. Introduction

5.2 Materials and Methods

5.3 Results

5.4 Discussion

5.5 Conclusion

6 General Discussion

6.1 Introduction

6.2 Biofunctionality assessment of annexin A5 and its importance for imaging 146

6.3 Biodistribution of radiolabeled annexin A5: how a pitfall remains a challenge 147

6.4 From imaging feasibility to treatment evaluation, and beyond..... 149

$\begin{array}{ll}6.5 \text { Future Perspectives } & \mathbf{1 5 0}\end{array}$

7 At last...

7.1 References

7.2 Summary

7.3 List of Publications

7.4 List of Abbreviations

7.5 Samenvatting

7.6 Dankwoord/Acknowledgments

7.7 Curriculum Vitae 


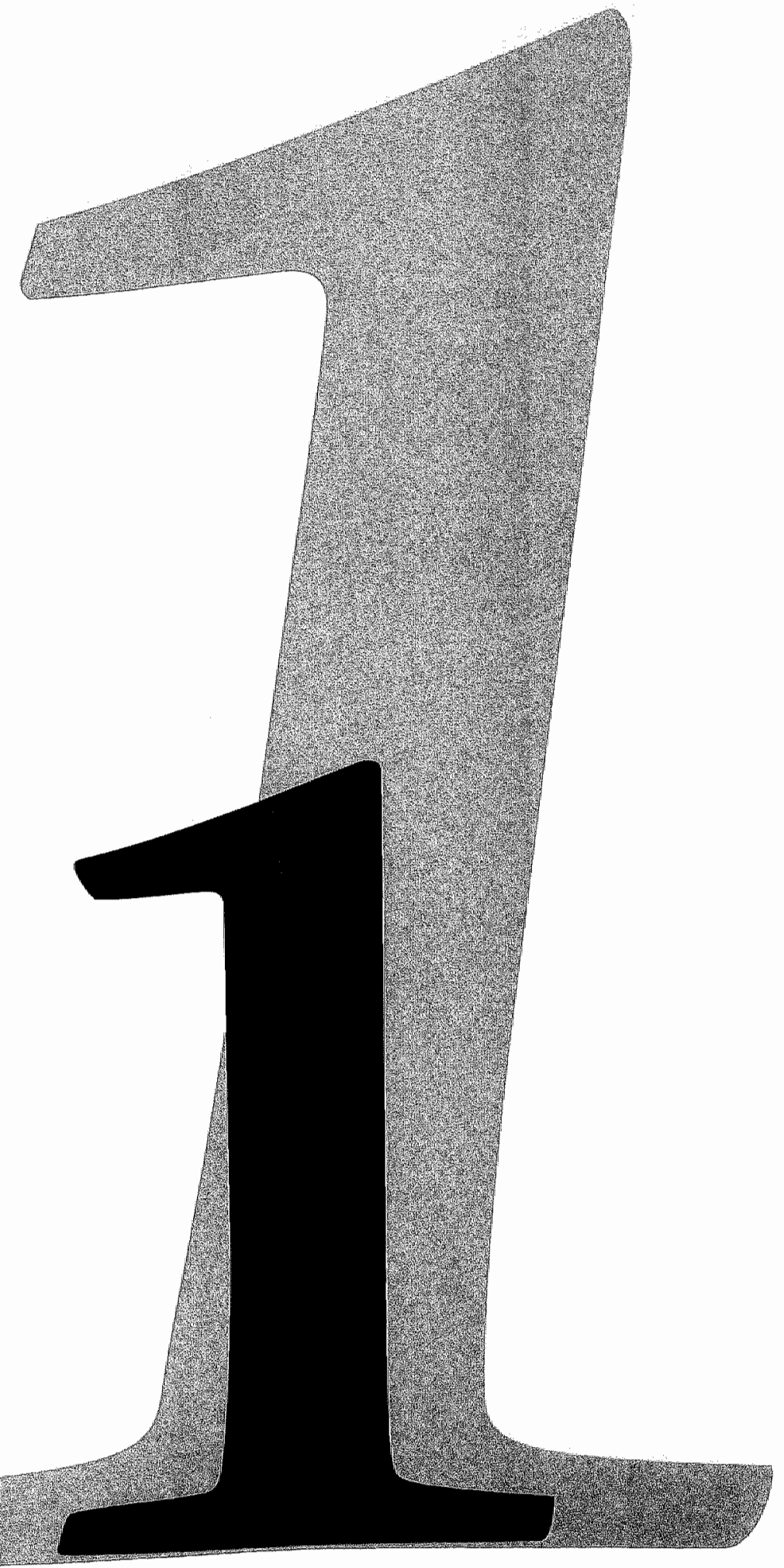





\section{General Introduction: Past, present and future of annexin $\mathrm{A} 5$ : from protein discovery to clinical applications}

Adapted from: H.H. Boersma, B.L.J.H. Kietselaer, L.M.L. Stolk, A. Bennaghwouch, L. Hofstra, J. Narula, G.A.K. Heidendal, C.P.M. Reutelingsperger. Past, present and furure of Annexin A5: from protein discovery to clinical applications. J Nuclear Medicine 2005; 46: $2035-50$ 


\subsection{Introduction}

The annexins are a multigene family of over 160 proteins which share the property of $\mathrm{Ca}^{2+}$-dependent binding to negatively charged phospholipid surfaces ${ }^{1}$. Annexin A5, one of the family members, binds to phosphatidylserine (PS). The latter is one of the "eat me" signals at the surface of the apoptotic cell. This characteristic has been the driving force for the research of annexin $A 5$ as a probe to measure apoptosis in vitro and in vivo. A non-invasive imaging procedure using annexin $A 5$ has been developed and applied successfully to measure programmed cell death (PCD) in patients. This chapter highlights the different aspects of this technique as a diagnostic tool and discusses radiopharmaceutical aspects, biodistribution, clinical relevance, limitations and future perspectives of this approach. 
The amino terminal of the molecule is unique for every annexin and is considered as the fingerprint to discern itself from other annexins. It differs in amino acid composition and in peptide chain length as well as in biological functionality ${ }^{8.14}$. Other features, related to the primary structure of annexin $A 5$ are:

- The annexin A5 molecule contains $30.7 \%$ charged amino acids, which is the basis for an isoelectric point of 4.8 . This is the result of combining 54 acidic (aspartic and glutamic acid) and 41 basic (lysine and arginine) amino acid residues ${ }^{\mathrm{a}}$.

- Annexin A5 possesses three types of calcium binding sites, i.e, type II (high affinity binding sites), type III and type B. These calcium binding sites consist of 1-3 carbonyl oxygens from the protein backbone strucrure, and one carboxy] group from an Asp or Glu residue ${ }^{15}$. The nature and properties of these binding sites are described in section 1.2.2.2.

- The polypeptide chain contains one cysteine residue, which allows for dimerization under conditions of denaturation. It has also been reported that about $2-4 \%$ of natural annexin A5 migrates as a dimer under non-reducing conditions on polyacrylamide gels. This suggests that the cysteine residue can also be involved in some intermolecular disulphide bond formation under physiological circumstances ${ }^{12}$. The single cysteine can also be used for labeling purposes $^{16,17}$, as will be discussed later.

* One single tryptophan residue is present in the third conserved domain on position 187. It has been suggested that this single residue is responsible for changing the conformation in an acidic environment $(\mathrm{pH}<4.6)$ and in the presence of low $\mathrm{Ca}^{2+}$ concentrations $(10-30 \mu \mathrm{M}, \mathrm{pH}>5)$. In either circumstance the annexin A5 molecule is able to bind most phospholipid bilayers. Under acidic conditions annexin A5 most likely penetrates the phospholipid bilayer, where it engages in interactions with the hydrophobic core of the membrane $e^{1,18 .}$ ${ }^{20}$ (see Chapter 2).

- At $\mathrm{pH}>5$, annexin $\mathrm{A} 5$ binding to membrane is of an extrinsic nature and depends on the presence of $\mathrm{Ca}^{2+}$-ions.

- Potential phosphorylation sites are present in the N-terminus of the annexin A5 molecule by the presence of two threonine residues. However, to date no phosphorylation of annexin $A 5$ has been reported.

* Annexin A5 lacks a signal sequence that is required for secretion via the known dassic secretory routes ${ }^{14}$. The presence of annexin A5 is reported in the extracellular space. Hence, other externalization pathways probably exist for annexin A5. Internalization of the molecule is possible. Its mechanism will be discussed in section 1.2.3. 


\subsubsection{Tertiary structure}

The tertiary structure of annexin $A 5$ was revealed by crystallisation of the molecule and subsequent $X$-ray crystallography ${ }^{21,22}$. The tertiary structure appears loyal to the primary domain structure. Each of the fout domain folds into a separate module in space. The four modules show high homology in their spatial organization ${ }^{23,24}$. All domains contain five stretches of $\alpha$-helices each (7-15 amino acids per helix, see Figure 1). The five $\alpha$-helices are wound into a right handed superhelix ${ }^{14,21}$. The modules are tightly packed giving the whole molecule a slightly curved shape with a convex and a concave side. The convex side contains the type II high affinity $C_{a}^{2+}$ binding sites, as well as the membrane binding site ${ }^{25}$. The concave side of the molecule is apical to the phospholipid-binding region and harbors the N-terminal tail of the protein. This position makes the tail accessible for interaction with soluble ligands, if annexin A5 is bound to the membrane (Figure 1.1).

\subsubsection{Biophysical properties}

Annexin A5 behaves as a peripheral membrane binding protein interacting reversibly with $\mathrm{PS}$ containing membranes ${ }^{26}$. This interaction is dependent on the presence of $\mathrm{Ca}^{2+}$-ions and $\mathrm{PS}$. $\mathrm{Ca}^{2+*}$ induces a conformational change in annexin $\mathrm{A} 5$ creating a binding pocket, which specifically interacts with the phosphoserine headgroup $2 \%$. Once bound to the surface, annexin A5 organizes in a two-dimensional crystal through protein-protein interaction. ${ }^{21}$. These interactions result in the formation of trimers, that constitute the basic building block of the crystal ${ }^{28}$. It appears unlikely that the interaction between annexin A5 and a phospholipid membrane is stereospecific. Each annexin A5 molecule covers ca. $5 \mathrm{~nm}^{2,26}$. Binding of annexin A5 to the cell membrane is said to be of an extrinsic nature. However; there is discussion about whether this is true under all conditions.

Isas et al postulated that annexin A5 builds ion channels in PS-containing membranes at mild ly acidic $\mathrm{pH}^{2 \%}$. Others suggest that the binding of annexin A5 to PS containing membranes is caused by simpler hydrophobic interactions, enabling penetration of annexin A5 into the phospholipid bilayer ${ }^{\forall 9}$. Urilizing paramagnetic beads, we observed binding of annexin A5 to PS-containing membranes in the absence as well as in the presence of $\mathrm{Ca}^{2+}$ at $\mathrm{pH} 3$ and $4^{20}$ (see Chapter 2 ).

Thus, we and others challenge the hypothesis that $\mathrm{pH}$-dependent binding to phospholipid membranes of annexin $\mathrm{A} 5$ in the absence of $\mathrm{Ca}^{2+}$ does not exist ${ }^{28}$. The

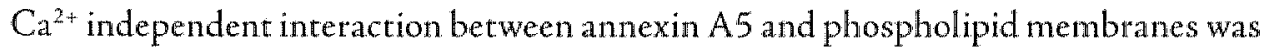
measured in vitro experiments. It remains unknown whether such interaction occurs in vivo.

The amount of PS availlable in the membrane and the physicochemical environment determine the $\mathrm{Ca}^{2 *}$-concentration needed for annexin $\mathrm{A} 5$ binding ${ }^{30}$. This is illustrated by the fact that membranes containing predominantly phosphatidyl choline (PC) 
require 10-100 $\mathrm{mM} \mathrm{Ca}{ }^{2+}$ to enable the binding of annexin A5. Adding PS and cardiolipine to these membranes lowers the amount of $\mathrm{Ca}^{2+}$ necessary for the binding of annexin $\mathrm{A} 5$ to $10-40 \mu \mathrm{M}^{12}$. The amount of $\mathrm{Ca}^{2+}$ required for annexin $\mathrm{A} 5$ binding increases when the amount of cholesterol is increased ${ }^{20,26}$. Furthermore, it has been shown that, using glass beads, coated with a phospholipid surface, the incorporation of phosphatidyl ethanolamine (PE) in the presence of PS decreases the amount of $\mathrm{Ca}^{2+}$ needed for the binding of annexin $\mathrm{A} 5$ to $\mathrm{PS}^{30}$.

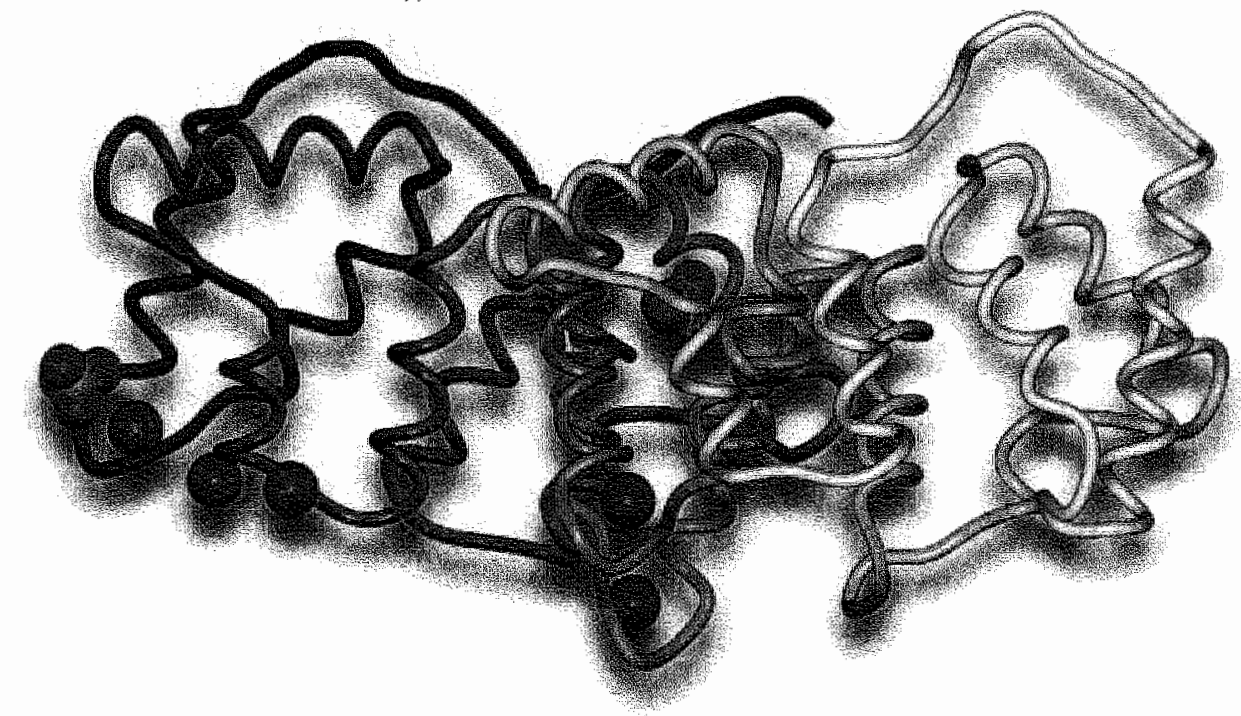

Figure 1.1: The tertiary structure of annexin $A 5$ was revealed by $X$-ray analysis. The protein consists of 319 amino acids. The molecule is arranged in a planat cyclic structure of four domains, which are indicated by different colors in the structure. The structure shown here depicts $\mathrm{Ca}^{2+}$ ions (green spheres) as well.

Crystal structures of annexin A5 have shown up to 10 bound calcium ions in three different types of binding sites, but previous work concluded that only one of these sites accounted for nearly all of the membrane binding affinity of the molecule. The type II calcium binding site in domain I was thought to be predominantly responsible for PS-binding ${ }^{31,32}$. Although domains II-IV also exert PS binding properties, it was shown that these properties are less efficient. This is likely to be due to steric hindrance of the formation of the protein- $\mathrm{Ca}^{2+}$.PS ternary complex ${ }^{33}$.

However, recently, mutants of annexin A5 were used to study the contribution of the calcium binding sites to PS binding ${ }^{3 *}$. The calcium binding site types II, III and $B$ in domain I-IV were the subject of research. This study showed that all four type II binding sites were necessary for high affinity binding. The latter was also shown for three of the four B sites (in domains I through III); the type III site in the first domain contributed marginally to the affinity of binding. Furthermore, binding 
experiments utilizing multi-site mutants showed that each domain contributed additionally and independently to binding affinity. However, binding affinity of type II and type B sites within the same domain were demonstrated to be interdependent. The number of functionally important binding sites identified correlated well with the Hill coefficient determined in calcium tirrations. This study reveals that a previously unknown role exists for type B calcium binding sites in the membrane binding of annexins and suggests that all four domains of the molecule are required for maximum membrane binding affinity.

Most of the cellular PS in viable cells is not expressed at the cell surface. Consequently, viable cells hardly bind annexin A5. In some processes the cell translocates PS to the outer leatlet of the plasma membrane. Examples of such processes include platelet activation, macrophage activation, erythrocyte aging, and programmed cell death. The latter feature is utilized for the detection of apoptosis using annexin A5, as will be discussed in section 1.2.6. Annexin A5 can also bind to oxidized PS ${ }^{35,36}$. Oxidation of PS may occur during apoptosis ${ }^{35,36}$. It was shown that the binding of annexin A5 to oxidized PS is still adequate and therefore, annexin A5 can still detect cell death under oxidizing circumstances ${ }^{36}$.

\subsubsection{Biochemical properties}

In addition to the biophysical properties, a large number of biochemical activities have been revealed for annexin A5. Almost all of these activities arise from the ability of annexin A5 to bind phospholipids.

In spite of this wealth of data, the exact physiological significance of annexin A5 remains elusive (See 1.2.5). The most important biochemical activities are highlighted below.

\section{Inbibition of blood coagulation}

One of the first biochemical properties discovered for annexin A5 was its ability to inhibit blood coagulation ${ }^{3}$. The underlying mechanism of this activity is indeed based. on the phospholipid binding action of annexin A5. The molecule is very effective in performing this particular action by inhibiting the prothrombinase complex $\left(\mathrm{IC}_{50}\right.$ $=16 \pm 12 \mathrm{nmol} / \mathrm{L})^{37}$. Recently, pregnancy losses were observed in patients with an antiphospholipid syndrome. Keeping in mind the anticoagulant properties of annexin $A 5$, these losses were subscribed to antibody-mediated inhibition of annexin A5 anticoagulant activity ${ }^{1.38}$.

\section{Inbibition of phospholipase $A_{2}$}

Phospholipase $\mathrm{A}_{2}$ enzymes (PLA ${ }_{2}$ ) catalyze the hydrolysis of the sn-2 fatty acyl chain in many phospholipids ${ }^{39,40}$. PLA 2 plays a key role in inflammatory processes because of its ability to release arachidonic acid from the lipids in the cell membrane. 
Subsequently, arachidonic acid is further metabolized by the cyclo-oxygenaseisoenzyme family and other inflammatory enzymes in order to generate compounds such as prostaglandins and leukotrienes ${ }^{41}$. Annexin $A 5$ was shown to inhibit PLA ${ }_{2}^{39.40}$ by preventing access of the enzyme to its substrate, negatively charged phospholipids ${ }^{32,39,42}$.

\section{Inbibition of protein kinase $\mathrm{C}$}

The Protein kinase $\mathrm{C}$ isoenzymes form a family of serine-threonine kinases, involved in signal transduction pathways, that catalyze numerous biochemical reactions critical to cellular homeostasis, as well as under disease conditions ${ }^{43}$. The enzyme activity is calcium activated and phospholipid dependent. Activation of protein kinase $\mathrm{C}$ is triggered by, for instance, the $\mathrm{G}$-protein coupled production of diacylglycerol. This is the physiological stimulator of protein kinase $\mathrm{C}$, and is responsible for protein kinase $\mathrm{C}$-mediated cell division.

It has been shown that annexin $A 5$ inhibits protein kinase $C$ in vitro, presumably by preventing the binding of phospholipids to protein kinase $\mathrm{C}^{1,44}$. Furthermore, in intact MCF-7 cells, it was found that the effects, normally induced by treatment of the cells with the protein kinase $\mathrm{C}$-activating phorbol esters, were inhibired by annexin A5. Hence, it is likely that this inhibition is mediated through suppressing the protein kinase $\mathrm{C}$ activity ${ }^{1,44}$. An extended study ${ }^{31}$ performed with annexin $\mathrm{A} 5$ mutants lacking one or more $\mathrm{Ca}^{2+}$-binding sites, revealed that the lack of binding site $\mathrm{I}$ (corresponding with mutant $\mathrm{M} 1$ ) resulted in a partial loss of the ability of annexin A5 to inhibit the protein kinase $C-\alpha$ isoform. This effect tended to be more pronounced when other $\mathrm{Ca}^{2+}$-binding sites were also inactivared. The authors hypothesized that the possible mechanism for protein kinase $C$-inhibition is the sequestration of phospholipids by annexin $A 5^{31}$.

\section{Annexin A5 interaction with integrins}

Integrins are ad hesion receptors, which are present in all multicellular organisms. These heterodimeric transmembrane proteins are pivotal in the development and survival of the organism ${ }^{45}$. They play an important role in the mediation of adhesion between cells and the extracellular matrix. Andersen et al described how annexin A5 was discovered to bind to the intracellular part of alpha(v)beta(3) and alpha(y) beta(5) integrins. Interestingly, annexin A5 turned out to be an impurity in the purification steps of the integrin. Annexin A5 binding to this type of integrin was shown to be phospholipid dependent ${ }^{45}$.

Activation of pinocytosis by annexin AS and subsequent internalisation of the molectle in cells expressing PS at their cell surface

The transportation of fluids across the external cell membrane is called pinocytosis. The mechanisms related to this process depend on the cell type 
and the connected biochemical pathways at a particular location in the cell. During apoptosis in macrophages macropinocytosis is induced by PS ${ }^{4}$. Upon expression of PS by the apoptotic cell, a hypothetical PS-receptor on the phagocyte is stimulated. This results in membrane ruffing and the formation of large fuid filled membrane particles. This process occurs when apoptotic cells are removed from its environment by macrophages ${ }^{47}$.

Very recently, it was shown that annexin $A 5$ activates a novel pinocytic pathway" The 2D-crystallisation of annexin A5 on the PS expressing membrane patch is the driving force for invagination and budding. The $2 \mathrm{D}$ protein network of annexin A5 at the surface prevents apoptotic body formation without interfering with the progression of apoptosis as demonstrated by activation of caspase-3, PS exposure and DNA fragmentation. The annexin A5 protein network bends the membrane patch nanomechanically into the cell and elicits budding, endocytic vesicle formation and cytoskeleton dependent trafficking of the endocytic vesicle. Internalisation of annexin $A 5$ has been described in vitro for a muscle cell line undergoing transient PS-expression ${ }^{49}$. Upon expression of PS, the cell line was incubated with fluorescently labelled annexin A5. Firstly, annexin A5 was shown to bind to the outer PScontaining cell membrane. Secondly, the cell gradually took up annexin A5. When the cells are depleted from $\mathrm{Ca}^{2+}$, there was only a partial loss of fluorescence intensity. It was observed with fuorescence microscopy that annexin $A 5$ appeared to be taken up by the cells. Moreover, it was shown that annexin A5 did not bind in the presence of $\mathrm{Ca}^{2+}$ to the outer cell membrane of PS-negative muscle cells, which had been PSpositive before. This novel pinocytic pathway differs from macropinocytosis, which is preceded by membrane ruffling and actin polymerization. It has been clearly shown that actin polymerization is not involved in budding and endocytic vesicle formation, but is required for intracellular trafficking. The phosphatidylserine-annexin A5 mediated pinocytic pathway is not restricted to cells in apoptosis. It has also been demonstrated that living tumor cells can take up substances through this novel portal of cell entry. This opens new avenues for targeted drug delivery and cell entry ${ }^{48}$.

\section{Calcium channel formation}

Generally, the annexins possess the ability to form calcium channels. For annexin A5, the latter was shown in B-lymphocytes at neutral $\mathrm{pH}^{50}$. The formation process of the ion channels is likely to be enhanced by exposure of these cells to physiological concentrations of peroxide. Other evidence for a role of annexin $\mathrm{A} 5$ as a $\mathrm{Ca}^{2+}$ channel is revealled by experiments using mineralizing chondrocytes ${ }^{51}$. Bone formation is mediated by $P S$-enriched matrix vesicles, which can entrap $\mathrm{Ca}^{2+}$ in order to be able to form calcium phosphate crystals. The observed $\mathrm{Ca}^{2+}$-channel activity shows many features in common with the annexin $\mathrm{A} 5 \mathrm{Ca}^{2 *}$-channel observed in vitro. For instance, they are blocked by $Z_{\mathrm{n}^{2}}{ }^{2}$, activated by $\mathrm{ATP}$ and inhibited by GTP. Furthermore, maximal $\mathrm{Ca}^{2+}$-influx is observed when annexin $\mathrm{A} 5$ is associated independently with 
the vesicles ${ }^{52}$. Also, the annexin A5-Ca ${ }^{2+}$-channel antagonist benzothiazepine $\mathrm{K} 201$ was shown to protect the rodent myocardium under $I / R$ conditions $s^{53}$. The latter suggests that annexin $\mathrm{A} 5$ is likely to play a $\mathrm{Ca}^{2+}$-channel role in the pathophysiological mechanisms of this group of diseases. Intracellularly, annexin A5 is thought to bind predominantly the cytosolic leaflets of the plasma membrane and several organelle membranes'.

\subsubsection{The role of annexin A5 in physiology and disease}

The role of annexin A5 in physiology is uncertain. As in vitro experiments and expression studies suggested a potential role for annexin A5 during calcification processes in vivo, new insights into the relevance of annexin $A .5$ in this process were gained. To study these aspects, an annexin A5-knock out mouse mutant was generated ${ }^{54}$. Mice lacking annexin A5 appeared to be viable and fertile, without any significant alterations in the biochemical parameters characteristic for metabolic or functional defects. Neither the development of skeletal elements nor the in vitro calcification properties of isolated chondrocytes is significantly impaired by the absence of annexin A5. Therefore, annexin A5 seems to be dispensable for basic homeostatic mechanisms and the formation and maintenance of skeletal elements in the mouse $e^{54}$.

In spite of a set of data about the correlation between annexin A5 and diseases, the role of annexin A5 in pathological circumstances is not evident to date. For instance, in both myocardial infarction ${ }^{55,56}$ (See also Chapter 3) as well as during end-stage heart failure ${ }^{57}$, annexin A5 plasma levels have been seen to rise. Interestingly, another group reported a similar rise of annexin A5 levels during heart failure, but this was restricted to non-muscle cells ${ }^{58}$. To date, no explanation has been found for these annexin A5 plasma level fluctuations.

In summary, it is evident that annexin $A 5$ interferes with a great variety of physiological and parhophysiological processes. In particular, it is believed that annexin A5 is likely to interfere with the calcium and phospholipid signaling pathway ${ }^{44}$, However, it is not yet understood whether all in vitro observed effects take place in a similar way and extend within the organism.

\subsubsection{Development of annexin A5 as a diagnostic tool}

In 1992, Fadok and co-workers revealed that PS is expressed on the outer leaflet of the plasma membrane of apoptotic cells, instead of staying on the inner cell membrane, which is usual in normal cell. $s^{59}$. Based on this experience, Koopman and others were the 
first to describe the use of fuorescently (FITC) labelled annexin A5 for the detection of programmed cell death ${ }^{60}$. To be able to discern between apoptotic cells and necrotic cells, the plasma membranes of which are no longer intact, propidium iodide (PI) must be added. By this means, viable, apoptotic and dead cells can be discriminated using either fluorescence microscopy of flow cytometry (see Figure 1.2).

This assay has been developed further by labeling annexin $\mathrm{A} 5$ with biotin to enable histochemical staining. Labeling with fluorescent (FITC, Oregon Green and Alexa568) and radioactive labels $\left({ }^{123} \mathrm{I},{ }^{124} \mathrm{I},{ }^{99 \mathrm{~m}} \mathrm{Tc},{ }^{18} \mathrm{~F}\right)$ can be applied to perform in vitro and in vivo experiments, respectively ${ }^{60-65}$. Furthermore, GMP-produced ${ }^{99 m} \mathrm{TC}$. annexin $A 5$ was used to measure apoptosis in humans ${ }^{55,6672}$. The latter subjects will be discussed further in section 1.4 .

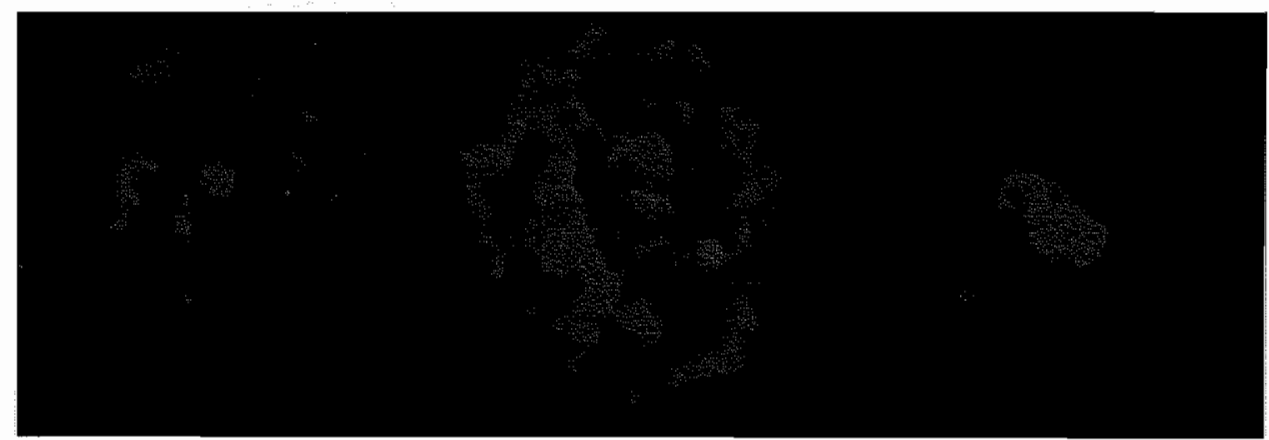
A
B
C

Figure 1.2: annexin A5 is able to bind cells in all stages of the cell death program. The pictures show FAS-ligand stimulated Jurkat cells. The situation in (A) illustrates the early stage of cell death. Fluorescent annexin A5 (green) binds to the outer cell membrane. (B) depicts a later phase showing binding of propidium iodicle (PI, red) which incercallates with the DNA and annexin A5, respectively. Binding of PI is indicative of the disintegration of the cell membrane. Also, the formation of apoptotic bodies is visible. The final stage of programmed cell death is shown in (C). Here, only annexin $A 5$ binding to cellular membrane residues can be observed. Adapted from ${ }^{73}$. 


\subsection{Intermezzo: basic principles of cell death}

Apoptosis, which is one of the major forms of programmed cell death $(\mathrm{PCD})^{74}$ plays an important role in the development, homeostasis and disease of an organism. In pathology two opposing situations can arise concerning apoptosis: one situation in which there is abundant apoptosis, as in cases of transplanted organ rejections, AIDS, septic shock and cardiovascular and neurodegenerative diseases and one in which there is insufficient apoptosis, as in some cases of cancer (Table 1.11) (15 $^{75}$ Alrhough, on the contrary, some tumor types, appear to have a high apoptotic index. During the past few years, evidence has been gathered that this feature correlates positively with the tumor malignanc $y^{71}$.

Table 1.1: Examples of abandonment and shortage of apoptosis, respecrively

\begin{tabular}{|ll|}
\hline Too much apoptosis & Too little apoptosis \\
\hline Myocardial/ Brain infarction & Leukemia \\
\hline Multiple sclerosis & Lymphoma \\
\hline Parkinson's disease & Colorectal cancer \\
\hline Sarcoidosis & Breast cancer \\
\hline Transplant rejection & Neuroblastoma \\
\hline
\end{tabular}

Apoptosis is characterized by a cascade of defined morphological and biochemical events. Morphologically, apoptosis results in cell shrinkage, mitochondrial swelling, condensation of the cells nucleus and membrane blebbing. Finally, the cell disintegrates into smaller apoptotic bodies, which are removed by phagocytosis ${ }^{76.78}$. One biochemical hallmark is the activation of caspases (cystein aspartate specific proteases). Caspase 3 is considered to be the executioner caspase, the activation of which marks the point of no return ${ }^{79}$. Intrinsic and extrinsic pathways can activate the caspase cascade. The intrinsic pathway can be activated by various stimuli including radiation, DNA-damaging cytotoxic agents, serum starvation and reactive oxygen species. These stimulilead to the release of the mitochondrial cytochrome $C$, which rogether with Apaf-1 and caspase 9 forms the apoptosome that activates caspase 3 (Figure 1.3).

The extrinsic pathway is activated by external signals such as Fas ligand and TNF, which bind to the so-called death receptors and thereby activate the caspase cascade (Figure 1.3). 
The extrinsic pathway can either directly activate caspase 3 or converge with the intrinsic pathway at the mitochondrion culminating in the activation of caspase 3 (Figure 1.3). Acrivation of caspase 3 underlies many of the apoptotic morphological features as well as the phenomenon of cell surface expression of $P S^{80,81}$.

Other forms of PCD appear to exist which are caspase-independent ${ }^{79}$. Leist and Jaattela proposed, on the basis of existing data, 4 distinguishable forms of PCD ${ }^{74}$. The current understanding is a sliding scale from apoptosis to a caspase-independent necrosis-like cell death type on the basis of morphology and biochemistry. Cell surface expression of PS appears to be the remarkable common denominator of the various forms of $\mathrm{PCD}^{74}$.

During apoptosis PS expression occurs downstream of cytochrome C release from the mitochondria". It happens early after the onset of apoptosis and it persists until the cell death program is fully completed ${ }^{5 \%}$. Cell surface expression of PS acts as an "eat me" signal for the phagocyte which removes the dying cell from the tissue before it causes an inflammatory response ${ }^{59,82}$. The proposed mechanism postulates a model of "tethering and tickling" in which PS causes the tickling through interaction with a receptor on the phagocyte ${ }^{47}$. Recently, cell surface expression of $P S$ in the absence of execution of the apoptosis has been observed by ourselves and others ${ }^{48,83}$. This process has been termed "apoptosis interruptus" or "escapoptosis". The latter event has been found to take place in murine B-lymphocytes ${ }^{48,83}$, as well as in vivo in mouse cardiomyocyte cells, exposed to a shorter time ( 5 min instead of $30 \mathrm{~min}$ ) of ischemia, followed by reperfusion. It has been found that after short, transient PS-exposure, annexin A5 was internalized in the cardionyocytes. Thus, annexin A5 is believed to be taken up by viable cells after short, transient PS-expression ${ }^{48}$. However, this does not so far imply that the longer lasting form of PS expression would be less indicative of the execution of the cell death program. 


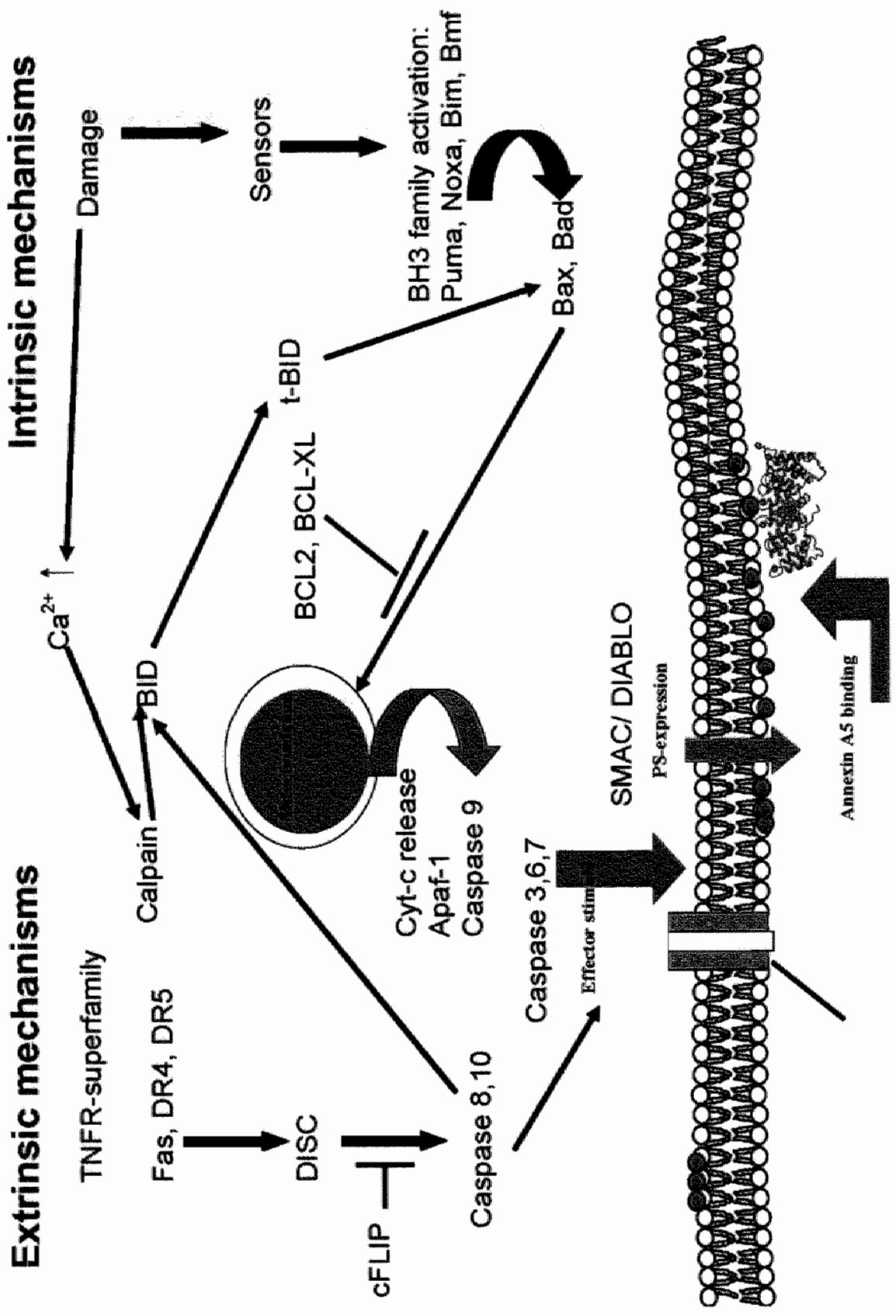

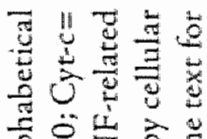

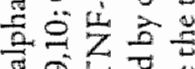

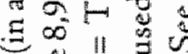

욜 岂

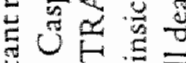

专

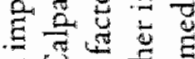

$50 \div$

ते

禹政

$0+5=4$

on已

을현

क क स

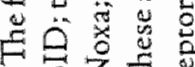

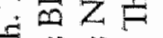

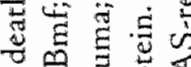

$\infty=0$

ㅂ

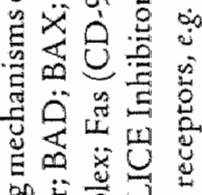

$\stackrel{5}{\leftrightarrows} \stackrel{2}{2}=$

$\exists$ 过焉

家

त

E

\&

$\circ \stackrel{3}{0}$

บ.

क

$\& 5$

E

iiv

4200

ये口

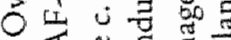

$\ddot{n} \Xi \pm E$

$\because \frac{5}{0}=\frac{\pi}{0}$

$\stackrel{2}{2} \stackrel{2}{2}$

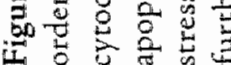




\subsection{Radiolabeled derivatives of annexin A5}

This section describes the state of the art of research with annexin A5 as a diagnostic tool. Firstly, the radioisotopes, their conjugation methods as well as the quality control of the radiopharmaceuticals are described. All main features, advantages and disadvantages of the isotopes used and corresponding radiopharmaceuticals are presented in Table 1.2 .

\subsubsection{Technetium-labeled annexin A5}

${ }^{99} \mathrm{~m} T \mathrm{c}$ is a radionuclide with good properties for diagnostic purposes. It has a short physical half-life (see Table 1.2), its radiation dose is low due to the absence of $\alpha$ and $\beta$ emission, and it provides images of good quality. The latter is a result of the favorable energy of its gamma radiation (Table 1.2), which enables good detection by the regular gamma camera equipment ${ }^{84}$. The complicated radiochemistry of ${ }^{99 \mathrm{~m}} \mathrm{Tc}$ can be regarded as one of its disadvantages. Unlike halogens, such as ${ }^{123} \mathrm{I}$ and ${ }^{18} \mathrm{~F}$, it is not possible to replace a hydrogen arom with a technetium ion, neither can it serve as a substitute for the commonly available carbon, nitrogen or oxygen atoms ${ }^{8.4}$.

Because of its more complicated radiochemistry, technerium requires a more complex conjugation principle to attach the isotope to bioactive molecules than, for instance, most halogens. Predominantly, the latter has been achieved by the use of bifunctional chelating agents. Several methods were utilized to label annexin A5: ester bonds (MAG3, dithiolate), amide bonds (HYNIC), and thiolate bonds (iminothiolane) ${ }^{85}$. Some preliminary data are available on the use of another isotope of technetium, 4Am Tc, as a PET-probe for the labeling of HYNIC-annexin A5. Because of its halflife and favorable positron energy (Table 1.2), its use as a PET radiopharmaceutical seems very attractive ${ }^{85}$. Labeling methods for technetium are well-established. A major problem with this PET-technetium in clinical studies may be the quality of the images as compared to the SPECT images. The rather poor biodistribution and the long biological half-life of ${ }^{99 \mathrm{~m}} \mathrm{TC}$-HYNIC-annexin A5 could be even worse for ${ }^{94 \mathrm{~m}} \mathrm{TT}^{\mathrm{C}} \mathrm{C}$ HYNIC-annexin A5, because of the shorter half-life of this isotope. Maybe other labeling methods using ${ }^{94 \mathrm{~m}} \mathrm{~T} \mathrm{c}$ will give better results in clinical practice. 


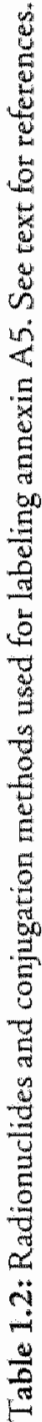

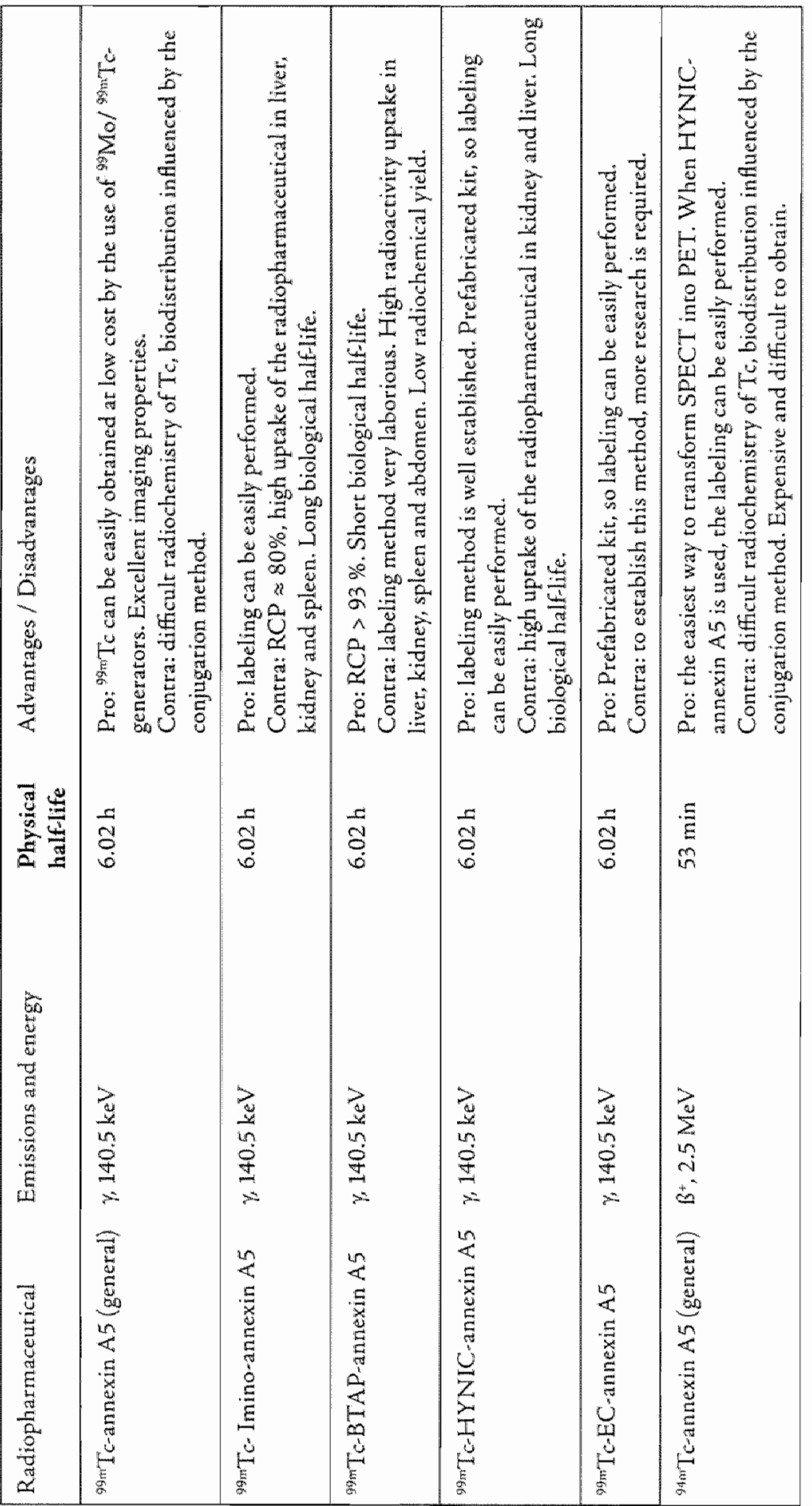




\begin{tabular}{|c|c|c|c|c|}
\hline 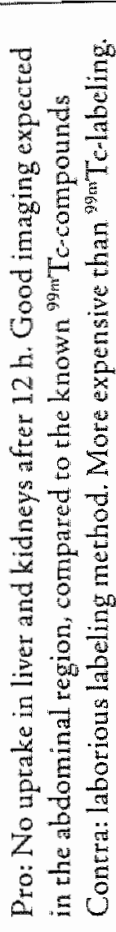 & 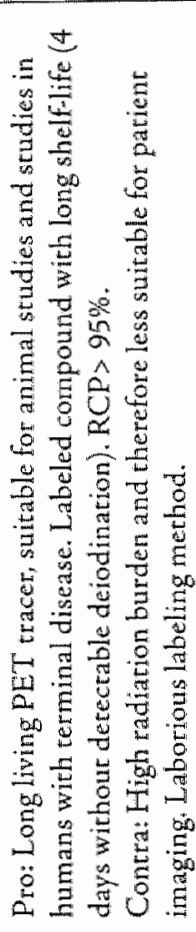 & 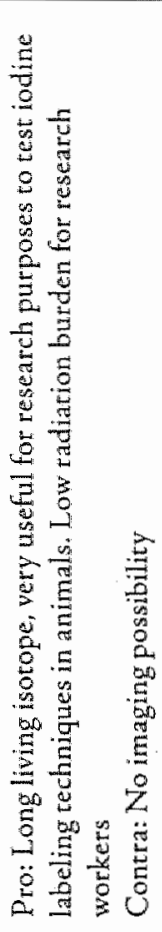 & 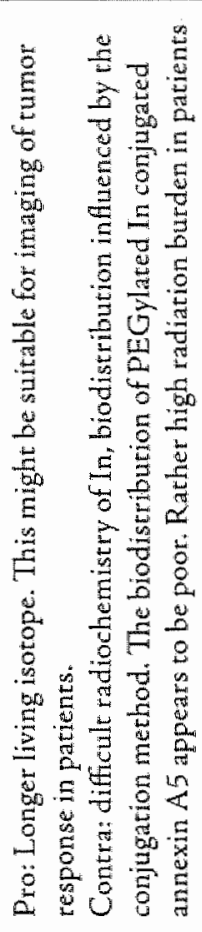 & 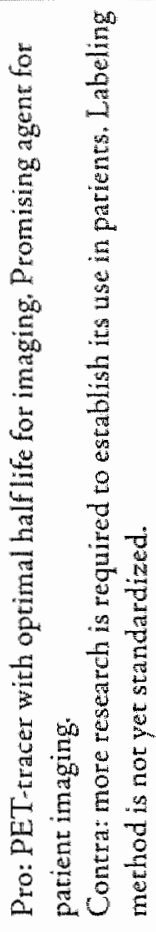 \\
\hline$\frac{r}{m}$ & $\begin{array}{l}\frac{n}{d} \\
\frac{d}{d} \\
\frac{d}{d}\end{array}$ & $\frac{n}{\frac{n}{8}}$ & 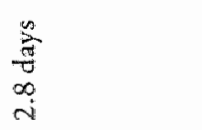 & $\stackrel{\underline{E}}{\stackrel{E}{E}}$ \\
\hline$\underset{⿱ 乛}{\frac{a}{8}}$ & 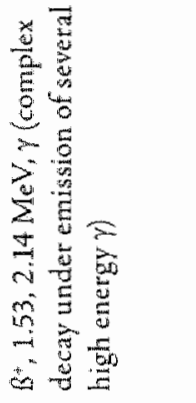 & $\begin{array}{l}\frac{3}{4} \\
\frac{1}{n} \\
0 \\
0 \\
0\end{array}$ & 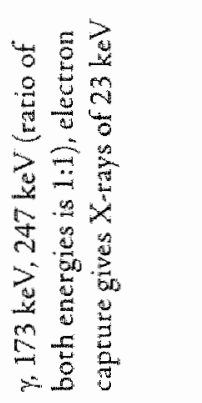 & 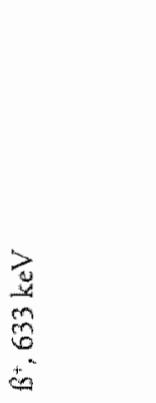 \\
\hline 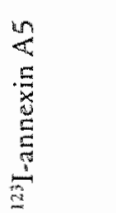 & 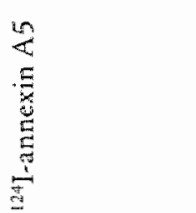 & 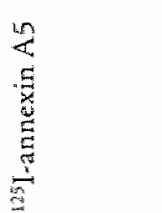 & $\underset{\Xi}{\stackrel{E}{E}}$ & 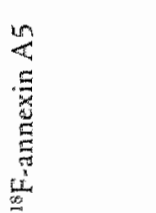 \\
\hline
\end{tabular}




\subsubsection{1 ${ }^{99 \mathrm{~m}} \mathrm{Tc}$-(n-1-imino-4-mercaptobutyl)-annexin A5}

Firstly, we started to perform the radioactive labeling of annexin A5 utilizing ${ }^{99 \mathrm{~m}} \mathrm{TC}$ (n-1-imino-4-mercaptobutyl)-annexin A5 (Imino-annexin A5). This conjugation method was developed at the R\&D department of Mallinckrodt and commercially used to label human immunoglobulin for the detection of inflammation ${ }^{86}$. Using iminothiolane, a radiolabeling kit was prepared for annexin A5, and was used for the first published studies in humans ${ }^{66,87}$. Iminothiolane converts aminogroups in the annexin A5 molecule into free sulfhydrylgroups. These can bind technetium in the presence of stannous ions. To achieve labeling of acceptable quality, a long incubation time $(2 \mathrm{~h} \text { ) is necessary (Table } 1.2 \text {, see Chapter } 3)^{55}$.

\subsubsection{2 ${ }^{99 m}$ Tc-BTAP-annexin A5}

We investigated the use of the dithiolate conjugation method to prepare (4,5-bis (thi oacetamido)pentanoyl)-annexin A5 ( ${ }^{90}$ Tc-BTAP-annexin A5, BTAP-annexin A5). This was a more accurate way to obtain ${ }^{99 n} \mathrm{Tc}$-annexin $\mathrm{A} 5$, but the labeling procedure was quite laborious (See Chapter 3 and 4). The entire preparation procedure took about 75 minutes. Another disadvantage was the rather fuctuating and low radiochemical yield (10-25\%), which depended predominantly on the specific radioactivity of the eluate used (H.H. Boersma, C.P.M. Reutellingsperger, G.A.K. Heidendal, 2002, unpublished results). The method to label technetium to annexin $\mathrm{A} 5$ was previously developed as the Onco Trac method to label Fab fragments for tumor detection ${ }^{88}$.

\subsubsection{3 ${ }^{99 \mathrm{~m}} \mathrm{Tc}-\mathrm{EC}$-annexin A5}

A method similar to the labeling of BTAP-annexin A5 is the use of ethylenedicysteine as a linker ${ }^{17}$. Like the BTAP method, conjugation of erhylenedicysteine was done at room temperature under basic conditions. However, the conjugate was purified by dialysis and then freeze dried in order to use it after a particular time to label technerium with this prefabricated kit. Labeling of EC-annexin A5 $(50 \mu \mathrm{g})$ with ${ }^{99 m \mathrm{Te}}$ was achieved in the presence of $100 \mu \mathrm{g}$ stannous chloride. Using this labeling method, a RCP of ca. $100 \%$ was achieved. This method seems an adequate and efficient way to label annexin A5 with technetium. It seems worthwhile to perform additional investigations with this labeling method.

\subsubsection{4 ${ }^{99 m}$ Tc-HYNIC-annexin A5}

Another possibility for labeling annexin A5 is the hydrazino-nicotinamide (HYNIC) conjugation method to connect technetium to the protein ${ }^{65}$. Until now, technetiumlabeled HYNIC-annexin A5 has been proven to be the mainstay annexin A5 radiopharmaceutical. It is a preformulated radiolabeling kit with a long shelf-life. In the presence of stannous ions, and tricine as a co-ligand, it results in HYNIC-annexin A5 
with a radiochemical purity of about $90 \%$ without requiring additional purification 20.89 (see also Chapter 2 and 5). Performance of the labeling is a one-step reaction with 30 min incubation at ambient temperature as the largest time consuming step. Hence, it is accurate and fast. It requires about $1 \mathrm{GBq}^{99 \mathrm{~m}} \mathrm{Tc}$, which is about $25 \%$ of the radionctivity needed for the preparation of BTAP-annexin A5. Furthermore, ir became available for clinical trials and was approved by the FDA as a New Investigational Drug.

Using ethylenediamine diacetic acid (EDDA) as a co-ligand, it should be possible to label technetium to HYNIC-annexin A5. However, this labeling is more complicated due to practical reasons (e.g. incubation of the reaction mixture at $37^{\circ} \mathrm{C}$ ) and the radiochemical purity is too low (35\% after $60 \mathrm{~min}$ incubation) for administration without further purification ${ }^{89}$.

\subsubsection{Novel labeling methods using ${ }^{99 \mathrm{~m}}$ Tc-annexin A5 mutant proteins}

To simplify the preparation and labeling of annexin A5 for imaging purposes, the addition of peptide sequences that will directly form endogenous chelation sites for $99 \mathrm{~m} T \mathrm{c}$ were investigated. Three annexin A 5 mutants, named annexin $\mathrm{V}-116,-117$, and -118 , were constructed with $\mathbb{N}$-terminal extensions of seven amino acids containing either one or two cysteine residues and expressed in Escherichia coli. The biopotency of these annexin A5 mutants was shown to be conserved. Using stannous chloride as the reducing agent and glucoheptonate as the exchange agent, all three proteins could be labeled with ${ }^{99}$ "Tc. Membrane binding activity of the labeled proteins was shown to be comparable to wild type annexin A5. The labeling reaction was rapid, reaching a maximum of approximately $93 \%$ RCP after 40 min at room temperature. Biodistribution of radioactivity was determined at 60 min after injection. Uptake of radioactivity was highest in kidney and liver, consistent with previous results obtained with wild-type annexin $A 5^{90-92}$. The authors show that annexin $A 5$ can be modified near its $N$-terminus to incorporate sequences that form specific chelation sites for $99 \mathrm{~m}$ Tc without altering its high affinity for cell membranes".

Similarly, the same group investigated whether the novel ${ }^{96}$ Tc-catbonyl labeling method would be suitable for annexin A5. Two mutant molecules of annexin A5, annexin V-122 and annexin $V-123$, were constructed with $N$-terminal extensions containing either three or six histidine residues. These molecules were expressed in E. Coli. Both mutant proteins retained full binding affinity for cell membranes with exposed PS. Using the $99 \mathrm{~m}$ Tc-carbonyl reagent, both proteins could be labeled with $99 \mathrm{mc}$ to specific activities of at least $3,7-7,4 \mathrm{MBq} / \mathrm{\mu g}$ with full retention of bioactivity. The radiolabeled proteins were stable when incubated with phosphate-buffered saline or serum in vitro, and there was no transchelation of label to serum proteins during in vitro incubation ${ }^{92}$. More research is necessary to establish the use of these annexin A5 mutants in humans. 


\subsubsection{Annexin A5 labelled with halogen radioisotopes}

Most halogens used in radiolabeling are suitable for conjugation with annexin A5. Labeling of th annexin $A 5$ with ${ }^{123} \mathrm{I},{ }^{124} \mathrm{I}$, and ${ }^{18} \mathrm{~F}$ has been described. This extends the possibilities for the use of radiolabeled anmexin A5 further towards PET as well as to other SPECT applications.

\subsubsection{1 ${ }^{123} \mathrm{I} /{ }^{124} \mathrm{I} /{ }^{125} \mathrm{I}$-annexin A5}

Actually, the first method described to label annexin A5 radioactively, was iodination using ${ }^{125} \mathrm{I}$ and later ${ }^{123} \mathrm{I}$. By then annexin $\mathrm{A} 5$ was being investigated as a thrombus identifying agent, as the occurrence of PS-expression on apoptotic cells had not yet been revealed ${ }^{93}$.

Several types of iodination of annexin $\mathrm{A} 5$ using ${ }^{123} \mathrm{I}$ and ${ }^{125} \mathrm{I}$ were described. The efficiency of iodo-beads iodination was just below 30\%; with the Bolton-Hunter protocol $40 \%$ efficiency was achieved. It was found that the iodo-beads method of labeling resulted in a protein that was rapidly deiodinated in vivo. In contrast, when annexin A5 was labeled using the Bolton-Hunter protocol, there was no evidence of unbound ${ }^{125} \mathrm{I}$ activity accumulating in the thyroid ${ }^{94}$.

Recently, Lahorte and co-workers have developed a slightly modified lodogen method ${ }^{95,96}$ and achieved the synthesis of ${ }^{123}$-annexin A5 with good radiochemical yield $(87.0 \pm 6.5 \%)$ and accurare labeling (radiochemical purity $>98 \%)$. But, much like the BTAP-annexin A5 labeling method, it involves elaborate preparation. However, because of its apparently rather favorable biodistribution properties for some applications, as will be discussed later, (see section 1.7.1) this type of labeled annexin $A 5$ seems of interest, especially for imaging the kidneys ${ }^{96}$.

For PET-purposes, the use of ${ }^{124} \mathrm{I}$-annexin $\mathrm{A} 5$ is currently under investigation. Because of its long half life (Table 1.2), ${ }^{124} \mathrm{I}$ is likely to be an excellent radioisotope for animal studies. The long thalf life is a problem however for studies in humans, because the irradiation received might be too high. Several labeling methods for ${ }^{124}$ I have been described. Until now, only one method was published as a full paper. Glaser et al. used two different methods for the labeling of ${ }^{124} \mathrm{I}$. The authors compared the direct and common Chloramine $T$ method with the indirect ${ }^{124} \mathrm{I}-\mathrm{m}$-SIB-labeling method. For rhe latter method a ${ }^{124} \mathrm{I}$-ligand was produced which in turn is incubated with annexin $\mathrm{A} 5$. The authors showed that the ${ }^{124} \mathrm{I}-\mathrm{m}$-SIB-labeling method yielded better results than the chloramine $T$ method. The ${ }^{124} \mathrm{I}-\mathrm{m}-\mathrm{SIB}-\mathrm{lab}$ beling method resulted in better binding properties for the labeled annexin A5 moleculle in vitro ${ }^{63}$. 


\subsubsection{2 ${ }^{18} \mathrm{~F}$-labelled annexin A5}

${ }^{18} \mathrm{~F}$ is a radioisotope with a favorable half-life (Table 1.2) for PET-imaging in humans. To conjugate annexin $\mathrm{A} 5$ with ${ }^{18} \mathrm{~F}$, Zijlstra et al developed an indirect labeling method ${ }^{97}$. They used $\mathrm{N}$-succimidyl-4-[1 $\left.{ }^{18} \mathrm{~F}\right]$ fluorobenzoate to label annexin A5. In vitro binding studies revealed that ${ }^{18} \mathrm{~F}$-annexin $\mathrm{A} 5$ shows equivalent results compared to unlabeled annexin A5. The disadvantage of an indirect labeling method using ${ }^{18} \mathrm{~F}$ is the short life of the isotopes which required high initial amounts of radioactivity to start the production of the compound in order to obtain enough activity for imaging purposes $^{97}$.

\subsection{3 ${ }^{111}$ In-PEG-annexin A5}

Recently, a method was developed to conjugate " In to annexin A5. "In has the advantage of a longer physical half-life as compared to $99 \mathrm{~m}$ Tc. Furthermore, the investigators made an attempt to extend the biological half-life of annexin A5 by adding a polyethyleneglycol (PEG, MW ca. 3400) moiety to the molecule. "They firstly prepared a thiocyanate-PEG-DTPA ligand, which was subsequently conjugated, to annexin $\mathrm{A5}$. The latter was purified and thereafter used for labeling with ${ }^{111} \mathrm{In}{ }^{98}$. No human studies have been performed up till now.

\subsubsection{Conclusion}

Until now, the most experience has been gained with technetium labeled annexin A5. Generally, the disadvantage of most conjugation methods for this radioisotope is its poor biodistribution. So far, the best candidate for SPECT imaging using technetium would be ${ }^{99 \mathrm{~m}^{\prime} \mathrm{T}} \mathrm{C}$-HYNIC-annexin $\mathrm{A} 5$. However, other agents are also promising, such as ${ }^{123} \mathrm{I}$-annexin $\mathrm{A} 5$. Much more experience needs to be gained in the field of PET annexin A5 imaging, because of its better resolution. Here, ${ }^{18} \mathrm{~F}$-annexin A5 could become a good tracer. 


\subsection{Quality control of radiolabeled annexin A5}

Quality control of radiolabeled annexin $A 5$ in a clinical setting is in general performed using thin layer chromatography (ITLC) or column chromatography. These methods however are only able to determine whether the labeling procedure was accurate and to not provide information about the biological properties of labeled annexin A5. We have therefore developed a rapid and simple QC-method using PS-coated paramagnetic beads. We dubbed this assay the ApoCorrect ${ }^{\text {tow }}$ assay ${ }^{20}$ (see Chapter 2). 


\subsection{Imaging applications for annexin A5 in animal studies}

The concept that annexin $\mathrm{A} 5$ binds to apoptotic cells in the presence of $\mathrm{Ca}^{2+*}$, was first proven in immortalized cell lines. These results were obtained in apoptotic Jurkat cells ${ }^{81}$. The question remained, however, what would happen to cells during programmed cell death in the complexity of the living organism. To test this concept, several animal models were developed to prove the assumption that annexin A5 is able to bind any cell within an organism undergoing programmed cell death.

\subsubsection{Embryonic development}

Firstly, a mouse embryo model was used. Because programmed cell death plays a pivotal role in the development of an organism, experiments focused on the disappearance of the interdigital webs during paw-development. This event takes place at a certain stage (E13). At this stage animals were first injected with biotinylated annexin A5 and then sacrificed. With the use of immunohistochemistry, annexin A5 was shown to be present in the interdigital webs, which is indicative for the presence of cells undergoing programmed cell death9 (see Figure 1.4).

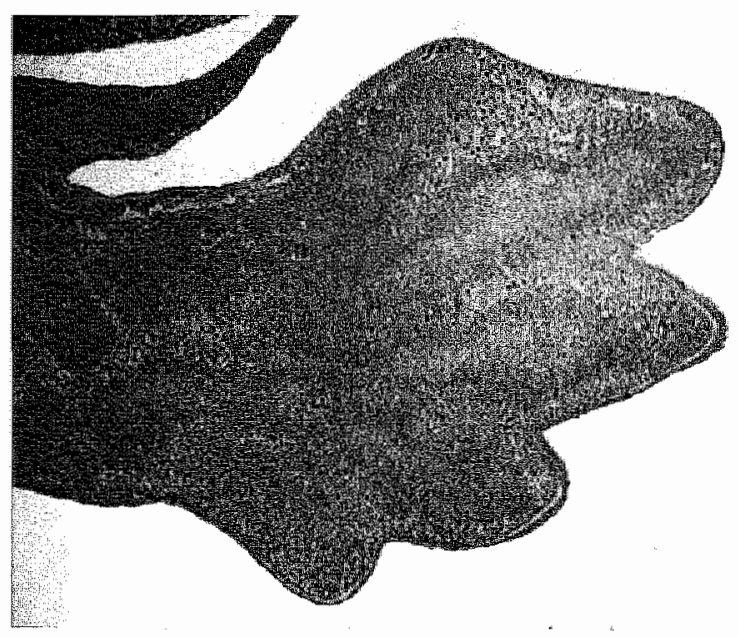

Figure 1.4: Paw of a mouse embryo at stage E13 of the animal's development. Biotinylated annexin $A 5$ can be observed in the interdigit space (black dots). Adapted from ${ }^{73}$. 


\subsubsection{Myocardial ischemia and reperfusion}

Another mouse model has been developed to mimick myocardial ischaemia and subsequent reperfusion. In this pathological model, the left anterior descending artery (LAD) was ligated (15-40 min). To obrain reperfusion, the ligation was removed $^{62,100}$ (see also Chapter $4 \& 5$ ). Prior to or just after the end of the ischemia, either biorin labeled, fluorescently labeled (Oregon green) or ${ }^{59 \mathrm{~m}} \mathrm{~T} \mathrm{c}$-annexin $\mathrm{A} 5$ were injected into the mice. The ligation of the LAD initiates cell death distal from the site of ligation, thus mimicking the situation of myocardial infarction and its abolition is similar to reperfusion strategies in humans. Dumont et al. showed that this model can indeed be used for the detection of cell death using either biotinylated (Figure $1.5 \mathrm{~A}$ ) or Oregon green labeled annexin $\mathrm{A} 5^{62,100}$ (Figure $1.5 \mathrm{~B}$ and $\mathrm{C}$ ).

Similar results were also shown by Taki et al. ${ }^{101}$, using ${ }^{99_{\mathrm{m}}} \mathrm{Tc}$-annexin A5. Moreover, they were able to demonstrate how the time course of the cell death process evolves from 30 minutes after restoration of the blood flow until 4 days and over. "They studied groups of rats after a 20 -min interval of coronary occlusion and reperfusion. At the time of the study, the rats were injected with ${ }^{99 \mathrm{~m}} \mathrm{Tc}$-annexin A5. One hour later, to verify the area at risk, ${ }^{201} \mathrm{TI}$ was injected just after the left coronary artery reocclusion and the rats were sacrificed. Dual-tracer autoradiography was performed to assess ${ }^{99 m}$ Tc-annexin A5 uptake and the area at risk. High ${ }^{99 m}$ Tc-annexin A5 uptake was observed in the mid myocardium after $0.5-1.5 \mathrm{~h}$ of reperfusion. The area of annexin A5 uptake had expanded througour the myocardium until $6 \mathrm{~h}$ after reperfusion and then gradually lessened over $3 \mathrm{~d}$. Similar to the data of Dumont et al..$^{62}$ in mice and also demonstrated by Thimister et al. ${ }^{68}$ in humans, these data indicate that annexin binding commences soon after ischemia and reperfusion in the myocardium within the area at risk and expands subsequently. The largest amount of radioactivity in the rat myocardium was observed at $6 \mathrm{~h}$ after reperfusion, followed by a decrease in activity over 3 days.

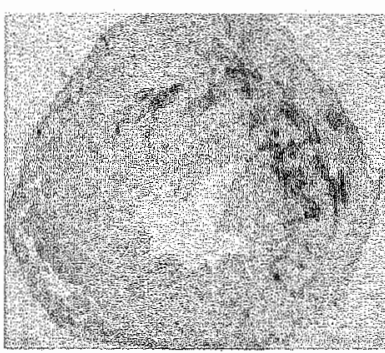

A

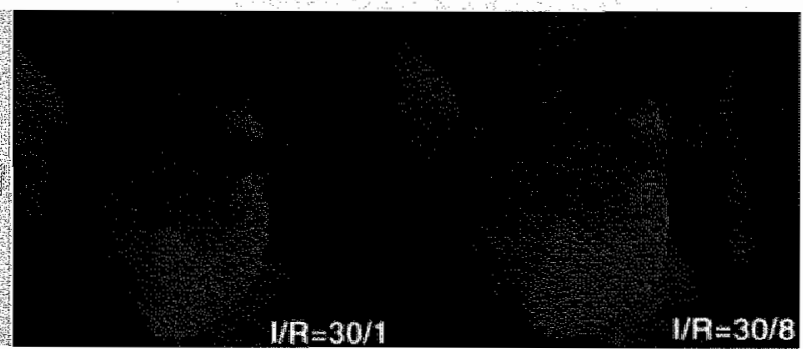

B

C

Figure 1.5: (A) Transverse section of a murine heart after $30 \mathrm{~min}$ of ischemia and $90 \mathrm{~min}$ of reperfusion (I/R 30/90). The cells were stained for the presence of biotinylated annexin A5. Fluorescent, in vivo whole heart imaging at I/R 30/1 (B) and I/R 30/8 (C) using Oregon Green labeled annexin A5. It is clearly shown that PS is expressed rapidly upon reperfusion. Figure $1.5(\mathrm{~A})$ was adapted from ${ }^{100}$, whereas Figure $1.5(\mathrm{~B}+\mathrm{C})$ was adapred from ${ }^{62}$. 


\subsubsection{Evaluation of cancer therapy}

Cyrostatic drugs and radiation therapy are widely used to treat cancer. As apoptosis is likely to be caused by these treatment types, the use of annexin A5 could be an option for treatment evaluation.

In animals, it was demonstrated that radiolabeled annexin A5 can be used for the evaluation of chemotherapy. Cyclophosphamide-induced apoptosis in vivo could be imaged with ${ }^{9 \mathrm{~m} T \mathrm{~T}-a n n e x i n} \mathrm{~V}-117$ (see 1.4.1.5). The group of Blankenberg and Strauss showed that ${ }^{99 m}$ To-HYNIC-annexin A5 is able to detect programmed cell death after administration of cyclophosphamide in lymphoma bearing mice ${ }^{65}$. External tadionuclide imaging showed a three to fourfold increase in the uptake of radiolabeled annexin $\mathrm{A} 5$ at sites of tumor apoptosis.

Mochizuki et al recently published a study in which they introduced hepatoma cells into the calf of rats ${ }^{102}$. Tumor cells were allowed to grow 11 days. Then, a single dose of chemotherapy was infused in one group, the controls teceived normal saline. After this, ${ }^{99 m}$ Tc labeled annexin A5 was administered. Witla the use of scintigraphy, the treatment group showed significant increase in annexin A5 uptake, when compared to controls. The results of increased apoptosis were confirmed by histological analysis, performing standard $\mathrm{H} \& \mathrm{E}$ staining and terminal deoxynucleotidyl transferase deoxyuridine triphosphate nick-end labeling (TUNEL) staining ${ }^{102}$. This finding could point to the fact that annexin A5 imaging after a single dose of chemotherapy can evaluate effectiveness of the therapy.

Recently, it was also shown that it is possible to administer ${ }^{99 \mathrm{~m}} \mathrm{Te}$-annexin $\mathrm{A} 5$ to tumor bearing rats repeatedly without any significant change in the biodistribution of the radiopharmaceuticals. So, it is likely that it is possible to follow the development of tumors and orher apoptosis-related processes in a rather narrow time frame withour affecting the imaging quality ${ }^{103}$.

As previously suggested, "111 In-PEG-anmexin A5 might be an option to enable long. term imaging of tumors. Recently, the imaging properties of an ${ }^{111}$ In-labeled, long circulating annexin A5 were evaluated in a mouse study. For this purpose, "'InDTPA-PEG-annexin A5 was prepared ${ }^{48}$. Imaging studies were performed in mice bearing subcutaneously inoculated human mammary tumors. The mice were treated with poly(L-glutamic acid)-paclitaxel, monoclonal antibody C225 (cetuximab), or a combination of poly (L-glutamic acid)-paclitaxel and C225, followed by intravenous injection of ${ }^{111} \mathrm{In}$-DTPA-PEG-annexin A5. Images were acquired $48 \mathrm{~h}$ after the injection of the radiotracer. The imaging properties of unPEGylated annexin $A 5$ and PEGylated ovalbumin were compared with those of ${ }^{11}$ In-DTPA-PEG-annexin A5. Tumor uptake of PEGylated "In-DTPA-PEG-annexin A5 $4 \mathrm{~d}$ after treatment was significantly higher in tumors treated with poly(L-glutamic acid)-paclitaxel and with combined poly (L-glutamic acid)-paclitaxel and C225 than in nontreated tumors, resulting in enhanced visualization of the treated tumors. ${ }^{111}$ In-DTPA-PEG-annexin 
A5 dispersed into the central zone of tumors, whereas ${ }^{111}$ In-DTPA-annexin A5 tumor uptake was restricted to the tumor periphery. Furthermore, uptake of ${ }^{111}$ In-DTPAPEG-annexin A5 by tumors correlated significantly with the apoptotic index ${ }^{104}$. A pitfall in the performed imaging is the thigh uptake of the radiopharmaceutical in liver and spleen. A similar problem was described for ${ }^{99 m}$ Tc-BTAP-annexin A5 in humans ${ }^{105}$ (see section 1.7.1 and chapter 3). In conclusion, increased uptake of and improved visualization with ${ }^{11} \mathrm{In}$-DTPA-PEG-annexin A5 in solid tumors after chemotherapy are mediated through both specific binding to apoptotic cells and nonspecific retention of macromolecular contrast agents in the tumors.

For the imaging of tumor response, Huorescently labeled annexin A5 was tried as well. In a study by Petrovsky er al ${ }^{106}$ "it was demonstrated that cyclophosphamide treatment of CR8 variants of Lewis Lung Carcinoma in mice caused a significant increase of the near infrared fluorescent $C \gamma$-annexin $A 5$ in the tumor.

\subsubsection{Other animal models}

Animal models of the research group of Blankenberg and Strauss show that HYNICannexin $A 5$ is able to detect programmed cell death caused by acute transplant rejection of the heart ${ }^{65}$, lungs ${ }^{107}$, liver ${ }^{108}$ of rats as well as in cerebral ischemia in rabbits ${ }^{109}$. In the rejection models, HYNIC-annexin A5 imaging showed a two- to sixfold increase in the uptake of radiolabeled annexin A5 at sites of programmed cell death. Immunohistochemical staining of cardiac allografts for annexin A5 revealed intense staining of numerous myocytes ${ }^{65}$. The increased annexin $A 5$ uptake in the lung transplant model was determined by a biodistribution assay (allograft uptake was significantly higher statistically, as compared to isograft activity ${ }^{107}$. In the model of cerebral ischemia, annexin A5 images showed multifocal brain uptake in both hemispheres of experimental but not in the sham operated control animals. Histology of the brains from experimental animals demonstrated scattered pyknotic cortical and hippocampal neurons. The absence of positive TUNEL staining is indicative of a necrotic-like cell death pathway. Double staining with markers of the cell type and exogenous annexin A5 showed that annexin A5 was localized in the cytoplasm of scartered neurons and astrocytes in the animals which underwent brain ischemia ${ }^{109}$. Furthermore, the occurrence of programmed cell death was demonstrated in an animal model of rheumatoid arthritis ${ }^{110}$. The a mount of PS-expression as a determination of severity of $\mathrm{PCD}$ in the front and rear paws of $\mathrm{DBA} / 1$ mice with type II collageninduced rheumatoid arthritis was shown to be almost 3 times higher than in the control group.

$\mathrm{C} 3 \mathrm{H}$ mice, given 5-Gy whole-body irradiation, showed a significant induction of apoptosis in the spleen, as measured by the TUNEL-assay, and a 4 -fold increase in ${ }^{125} \mathrm{I}$ activity (corresponding with the presence of ${ }^{125} \mathrm{I}$-annexin A5) in the spleens relative to that of the control animals ${ }^{94}$. 
Programmed cell death imaging in inflammation was further evaluated in an animal model of myocarditis, in which enhanced uptake of radiolabeled annexin $A 5$ was shown in the hearts of animals with an active myocarditis. This myocarditis was triggered by an immunization of rats by infusing porcine cardiac myosin. The animals formed antibodies against the myosin, and developed myocarditis, subsequently. Although the imaging in this model was done ex-vivo, a correlation was shown between severity of the myocarditis and the amount of annexin A5 uptake $e^{111}$.

Another example of "Tc-annexin A5 uptake in a myocarditis model was demonstrated by Peker et al ${ }^{112}$. They compared the myocardial uptake of ${ }^{996}{ }^{9} \mathrm{TC}-$ annexin A5 (imaging and quantitative autoradiography) using a experimental subacute myocarditis model in rats. The experimental model utilized an inbred strain of Wistar (Bonn/Kobori) rats. These rats spontaneously develop myocardial injury mimicking subacute catecholamine-induced myocarditis. Normal Wistar rats were used as control animals. Using scintigraphy, immunohistochemistry and autoradiography, this study showed that rats suffering from subacute myocarditis showed enhanced ${ }^{99}$ Tc-annexin A5 myocardial uptake ${ }^{112}$.

In conclusion, both imaging and monitoring of programmed cell death utilizing labeled annexin A5 is likely to be feasible in animal models. When the concepts tested in animals can be translated to humans, a more tational use of treatment options will become available. 


\subsection{Biodistribution and pharmacokinetics of radiolabeled annexin $\mathrm{A} 5$ in humans}

\subsubsection{Technetium labeled annexin A5}

To investigate and to compare the suitability of two types of ${ }^{90 \mathrm{~m}} \mathrm{Tc}$-annexin $\mathrm{A} 5$ for in wivo scintigraphy of apoptotic cells, the pharmacokinetics and biodistribution of two radiopharmaceuticals, ${ }^{90 \mathrm{~m}} \mathrm{Tc}$-(n-1-imino-4-mercaptobutyl)-annexin A5 (Iminoannexin A5) and ${ }^{99 m} \mathrm{Tc}-(4,5$-bis(thioacetamido)pentanoyl)-annexin A5 (BTAPannexin A5), were studied ${ }^{55,87,805}$ (see chapters 3 and 4).

99m Te-hydrazinonicotinamido (HYNIC)-annexin A5 was also investigated to assess safety, biodistribution and radiation dose ${ }^{90}$. Six healthy, male volunteers entered the study. About $250 \mathrm{MBq}$ of HYNIC-annexin A5 were injected intravenously. Subsequently, whole-body scans were obtained $30 \mathrm{~min}-24 \mathrm{~h}$ after injection (Figure 1.6). Organ uptake was determined. It was shown that the kidneys accumulated ca 50 $\%$ of the injected dose at $3 \mathrm{~h}$. after injection. Furthermore, the tadiopharmaceutical was taken up by the liver, the red marrow and the spleen, respectively. Almost all blood activity was cleared with a half-life of about $24 \mathrm{~min}$. The biological half-life of the radiopharmaceutical was long (ca. $70 \mathrm{~h}$ ). Excretion of the activity was predominantly through the urine ( $\mathrm{ca} 23 \%$ at $24 \mathrm{~h}$ ), and hardly any activity was seen in the bowel or in the feces. No adverse effects were observed in the healthy volunteers. The authors conclude that HYNIC-annexin A5 is a safe radiopharmaceutical, having a favorable biodistribution, better than BTAP-annexin A5 and Imino-annexin A5, for the imaging of apoptosis with an acceptable radiation dose. 


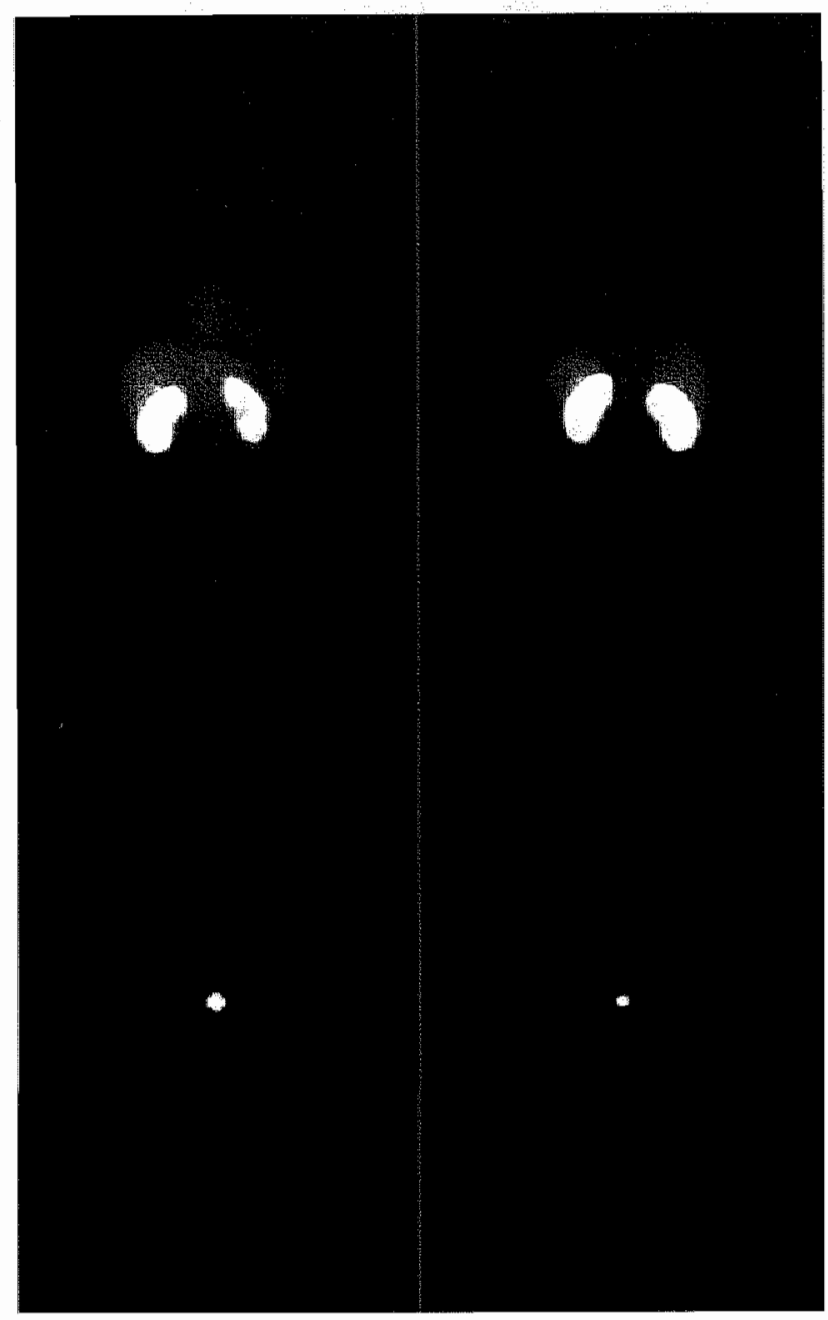

A

$\mathbf{P}$

Figure 1.6: View of a whole-body scan of a male volunteer (22y) raken after administration of $263 \mathrm{MBq}$ HYNIC-annexin A5, respectively ${ }^{90}$. A: anterior view; $\mathrm{P}$; posterior view. Adapted from ${ }^{90}$. 


\subsubsection{Iodine labeled annexin}

The ${ }^{123} \mathrm{I}$-annexin A5 biodistribution in human volunteers was investigated by Lahorte and others. They administered the radiopharmaceutical to 6 human volunteers. There seems to have been some initial imaging problems due to uptake of the radiopharmaceutical in the heart abdominal region and thyroid ${ }^{96}$. However, $21 \mathrm{~h}$ after administration, a rather low overall uptake in most organs was obrained. Theoretically, this is promising for the imaging of apoptotic processes in the kidney, such as transplant rejection. Furthermore, the longer half-life $(13 \mathrm{~h})$ of ${ }^{123} 1$ enables imaging to be scheduled longer after administration when compared with ${ }^{99 \mathrm{~m}} \mathrm{Tc}$. This labeling method still needs more investigation. 


\subsection{Past}

\subsubsection{Discovery}

At the end of the 1970's, annexin A5 was firstly isolated from the placenta and was named placental protein 4 (PP4) ${ }^{2}$. A few years later, the protein was isolated from human umbilical cord arteries and was given the name vascular anticoagulant protein alpha (VAC- $\alpha$ ) because of its inhibitory activity on blood coagulation ${ }^{3}$. Other groups isolated the protein as well and gave it a variety of names. In 1990 the annexin nomenclature was adopted by which the protein was named annexin V. None of the previous names did justice to all the properties that these proteins exhibited ${ }^{4}$. During the last decade more than 100 annexin family members have been discovered. The annexin nomenclature was adapted further by the introduction of five major annexin groups $(A-E)^{1}$. In this system, human annexin $V$ was renamed annexin A5.

Annexin $A 5$ has been identified in a broad range of tissue types such as the placenta ${ }^{2}$, spleen $^{5,6}$, liver ${ }^{6,7}$, uterus ${ }^{7}$, skin, kidney, heart and skeletal muscle of human origin ${ }^{6}$. After the discovery of annexin $A 5$, the human recombinant form was produced by the expression of its $C D N A$ in E. Coli bacteria ${ }^{8 \cdot 10}$. The CDNA was either isolated by screening cDNA libraries from placental or blood lymphocyte origin ${ }^{9,11}$ as well as by cDNA screening using monoclonal or polyclonal antibodies ${ }^{12}$.

\section{2 .2 The structure of annexin A5}

\subsubsection{Primary structure and functional aspects}

Cloning the cDNA of annexin A5 revealed that the protein consists of 319 amino acids forming a single polypeptide chain. The molecular weight is $35.7 \mathrm{kDa}^{10}$. See Figure 1.1. Like all annexins, annexin $A 5$ is composed of two major domains: the $\mathrm{NH}_{2}$-terminal tail, which is unique for each annexin, and the $\mathrm{COOH}$-protein terminal core domain, which is conserved among the annexins'. The core domain consists of 4 homologous repeats, each of which contains $65-70$ amino acids. This canonical repeat organisation is shared by all annexins. The repeats are connected by small linker peptides. Each repeat contains a consensus sequence of about 17 amino acids termed the endonexin loop and is presumed to be directly involved in phospholipid binding ${ }^{113}$. 


\subsection{Current clinical nuclear medicine applications}

In vivo imaging of cell death is an example of molecular imaging. This can be defined as an in vivo characterization and measurement of biological processes at the molecular level using imaging procedures. Molecular imaging of cell death tries to visualize one of the key components of homeostasis and disease, i.e. the detection of cells performing a cell death program.

We and others used the high affinity of labeled annexin A5 to cells expressing PS in order to detect cell death on a macroscopic level in different types of diseases. Until now, results in patients were obtained using ${ }^{9 m}$ Tc-annexin A5.

\subsubsection{Cardiovascular diseases}

Cell death in cardiovascular disease

An increased occurrence of PCD is common in cardiovasculat. diseases. Myocardial infarction, heart failure and atherosclerosis are typical parhological processes in which apoptosis plays a dominant role. There is a difference between cell death in acute processes, like myocardial infarction and the chronic forms of programmed cell death as in heart failure, for instance, Myocardial infarction is ultimately more likely to cause necrosis rather than apoptosis. This is caused by intense energy exhaustion from the initial apoptotic cascade, as it is postulated that terminally differentiated cells are very sensitive to adenosine triphosphate depletion. However, when the infarction ischemia is followed by reperfusion, the necrotic pathway is often remodulated into apoptotic cell death ${ }^{113}$.

Cell death in chronic cardiac disease is not a straightforward process as apoptosis may occur without the execution phase leading to cell death. Apoptosis occurs in the latter process through cy tochrome $c$ release, caspase 3 and caspase 8 activation (Figure 1.3). However, the cell nucleus remains intact and the cell is kept alive. This cellulat state is called apoptosis interuptus. These cells may eventually die from necrosis, or they continue their lives dysfunctionally, like zombie myocytes ${ }^{113}$.

\subsubsection{Myocardial infarction}

We performed clinical studies in patients with myocardial infarction and with subsequent reperfusion after undergoing percutaneous transluminal coronary angioplasty (PTCA) ${ }^{6,68}$. We were able to perform images of perfusion (using "9.m. sestamibi, ${ }^{96 m}$ Tc-tetrofosmin or ${ }^{201}$ Thallium) as well as cell death using ${ }^{99 \mathrm{~m} r}$ Tc-annexin A5. Under these circumstances, the infarcted area seen on a perfusion image strongly correlates with the area of increased uptake of BTAP-annexin A5 (Figure 1.7) Furthermore, we showed that the uptake of ${ }^{9 m} \mathrm{~T}_{c \text {-annexin }} \mathrm{A} 5$ in the infarcted area lasted up to four days after acute myocardial infarction ${ }^{68}$. These areas of annexin A5 uptake in myocardial infarction probably indicate preventable cell. loss, as shown 
in the mice study of Dumont et al. ${ }^{100}$ Arguably "rescued" cardiomyocytes can again regain their function and thereby provide a better outcome after acute myocardial infarction. Whether or not this is true in the human situation and whether annexin A5 can be used as an endpoint to measure cell loss is not yet known. Investigations are ongoing in this area.

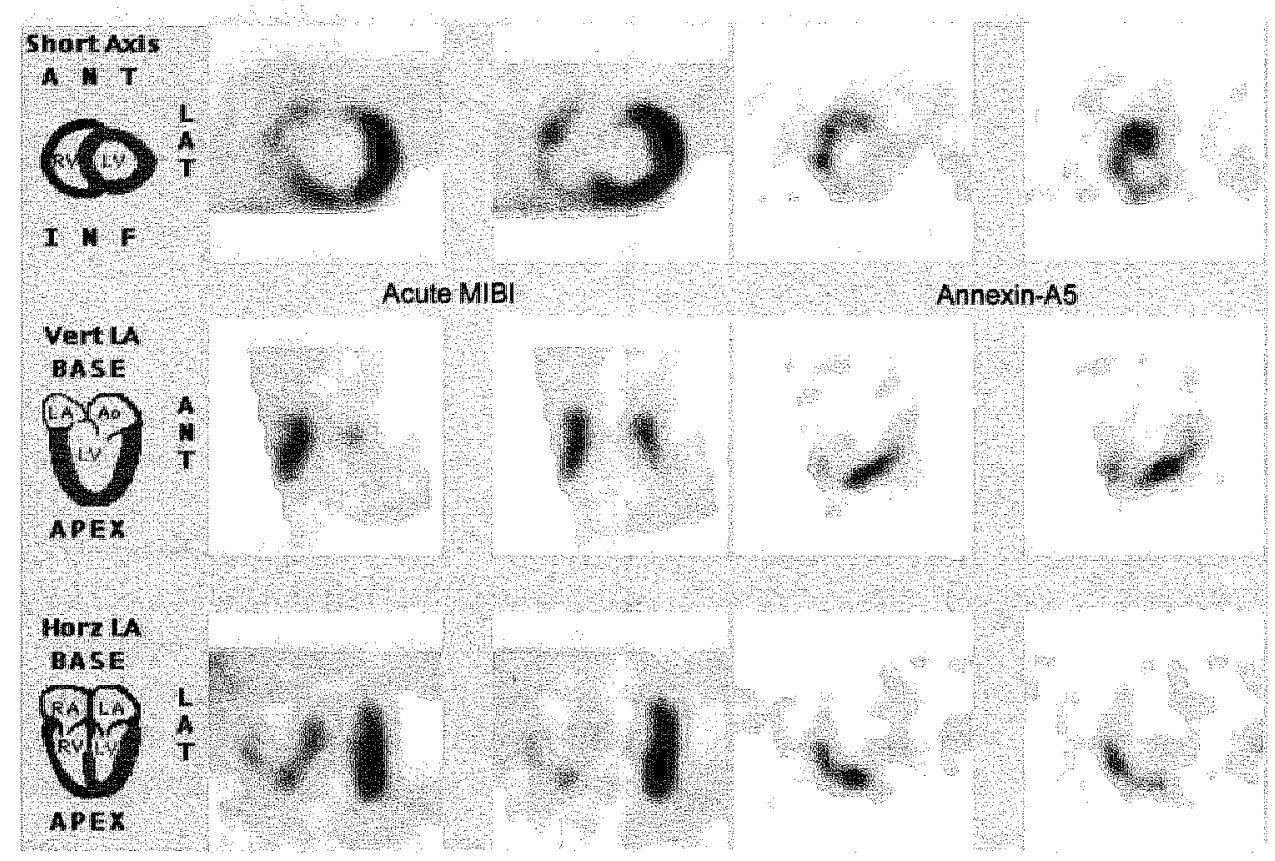

Figure 1.7: The left panel shows a MIBI scintigraphy in which the area of perfusion is depicted ${ }^{68}$. The latter gives an indication of the area at risk. Binding of ${ }^{99 \mathrm{~m} T} \mathrm{~T}$-annexin $\mathrm{A} 5$ to the reperfused myocardium is shown in the right panel; this is indicative for the presence of programmed cell death. Adapted from ${ }^{114}$.

\subsubsection{Cardiac allograft rejection}

A second application of molecular imaging of programmed cell death with radiolabeled annexin A5 was first described in 2002 by Narula and et al ${ }^{72}$. This study focused on cardiac allograft rejection in transplant patients. The diagnosis of rejection of the donor heart poses a difficult clinical problem. Early diagnosis is important because rejection can eventually be influenced by medication that suppresses the inflammatory response to the donor heart. Re-operation could be another possible consequence. On a cellular level, cardiac allograft rejection is histologically characterized by infiltration of monocytes in perivascular and interstitial spaces. This in turn causes 
myocyte necrosis and apoptosis. The common way to monitor possible rejection of the transplanted heart is to take myocardial biopsies, and scan for these morphological features. This procedure is, however, an invasive diagnostic test, which is not without risks. Since these processes have to be monitored over time, multiple biopsies have to be taken, adding to the risk of complications. In addition, biopsy taking introduces the risk of sampling error. SPEC ' ${ }^{-1}$ imaging of programmed cell death with labeled annexin A5 could possibly provide a non-invasive method for identifying patients suffering from cardiac allograft rejection. As mentioned previously, Blankenberg et al. had already proven the feasibility of this concept in an animal model of cardiac allograft rejection ${ }^{65}$. In the study of Narula et al. all patients $(n=18)$ underwent an annexin A5 SPECT study, and biopsies were taken. When assessing the SPECT images, two blinded observers were in perfect agreement about the uptake of radiolabeled annexin A5 in the myocardium of 5 of these patients. In 3 cases focal uptake, and in 2 cases general uptake of labeled annexin A5 was observed in the left ventricle. Tissue sections of the myocardial biopsies were assessed by standard $\mathrm{H} \& \mathrm{E}$ staining, TUNEL staining and staining for activated caspase 3 . H\&E staining revealed no or only limited abnormalities in the patients that showed no uptake of the radiolabeled annexin A5. Also, no activation of caspase 3 was observed. In those cases showing focal uptake, more severe abnormalities were seen in the H\&E staining, when scoring according to the recommendations of the International Society of Heart and Lung Transplantation (ISHLT). Also, scattered cardiomyocytes showed evidence of activation of caspase 3 . The 2 patients that showed general uptake of the labeled annexin A5 were scored as severe transplant rejection reaction according to the ISHLT guidelines, and showed many cardiomyocytes with activated caspase 3. TUNEL staining was positive in all but 2 patients. These data, together with the previously reported data on cardiac allograft rejection in animals, provide a proof of concept for the detection of programmed cell death in patients with cardiac allograft rejection. This could provide the clinician with an important imaging modality to identify patients at risk, to monitor therapy and to assess the efficacy of new treatment modalities such as cell death blockers in graft rejection ${ }^{72,115}$.

\subsubsection{Heart failure}

Recently, we investigated the feasibility of ${ }^{99 \mathrm{~m}} \mathrm{Tc}$-annexin A5 imaging in patients suffering from heart failure. This disease is defined by a decrease of myocardial functionality, which leads to upregulation of compensatory mechanisms. These, in turn, cause additional damage to the heart's function ${ }^{16,117}$. Functional cell death and loss of heart muscle cell functionality are believed to be important pathological conditions in heart failure ${ }^{118}$. Hence, PS expression is very likely to occur, so ${ }^{99 m} \mathrm{TC}_{\mathrm{C}}$ annexin $\mathrm{A} 5$ imaging has been evaluated as a diagnostic tool. In the study, nine patients 
with severe congestive heart failure were investigated (the left ventricular function was less than 0.35). SPECT-images from five of the nine patients showed myocardial

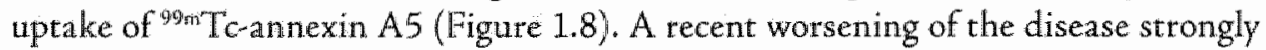
correlated with uptake of ${ }^{99 m}$ TC-annexin A5 in the myocardium, as all annexin A5negative patients had stable disease and all positive patients were progressive. So, despite the difference in etiology of the congestive heart failure of the investigated patients, it seems likely that ${ }^{99 \mathrm{~m}} \mathrm{TC}$-annexin A5 imaging is able to discern patients with accelerated myocardial cell loss. This kind of imaging may offer a new option for intervention in order to be able to prevent the loss of cardiac function ${ }^{118}$.

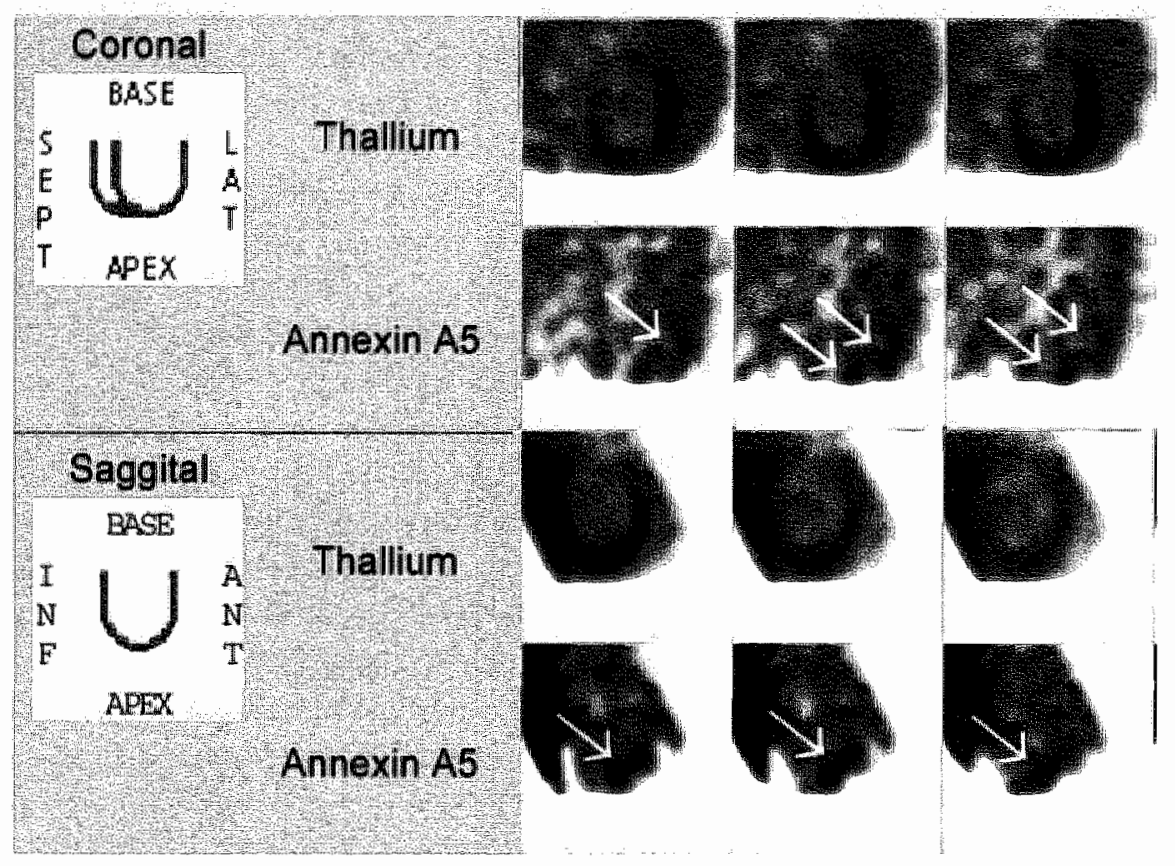

Figure 1.8: An example of 9 wh Tcannexin A5 uptake in a patient suffering from heart failure. The image shows multifocal annexin $A 5$ uptake in a patient with idiopathic dilated cardionyopathy. Dual isotope scan. Top rows: ${ }^{201}$ Thallium images for orientation purposes. Bottom rows: corresponding annexin A5 imaging showing multifocal uptake (artowheads). Adapted from ${ }^{118}$. 


\subsubsection{Atherosclerosis}

It has been also shown that imaging of PS-expression is possible in unstable artherosclerotic regions. Apoptotis was linked before to artherosclerosis ${ }^{119}$. Moreover, using animal models, we proved the feasibility of $99 \mathrm{~m} / \mathrm{T} c$-annexin A5 uptake in artherosclerotic plaques ${ }^{120}$. It would be clinically important to differentiate stable from unstable plaques especially in the carotid artery. The ustable plaques, showing increased apoptosis, could develop thrombosis with subsequent cerebrovascular accidents.

So, ${ }^{99 m}$ Tc-annexin A5 imaging in patients with atherosclerotic lesions in the carotid artery could be of value for non-invasive examination of their apoptotic status. In our $99 m \mathrm{~T}$-annexin A5 parient study for evaluation of artherosclerotic plaques, we imaged four patients with a recent or remote history of transient ischemic attack. Imaging was performed prior to removal of the patient's carotid lesions. The images and histology examination of removed artherosclerotic carotids of the patients (Figure 1.9) revealed that annexin A5 uptake in the lesion correlates highly with plaque instability ${ }^{121}$. This is illustrated by ${ }^{99 m} \mathrm{~T} T$-annexin A5-negative controls taken from the same patients. We removed artherosclerotic lesions without evident ${ }^{99}$ Tc-annexin A5 uptake. Histology of these lesions showed a stable plaque structure. Conclusion of this pilot study is that imaging of artherosclerotic plaques using ${ }^{99 n}$ Te-annexin A5 might help to identify plaque instability ${ }^{121}$. Further research using more patients is ongoing to establish this method. In conclusion, animal models and human studies provide evidence for the assumption that molecular imaging of programmed cell death using radiolabeled annexin A5 might provide useful information for diagnostic and therapeutic clues of disease states that are characterized by an excess of cell death, such as graft rejection in other transplanted organs and, as previously mentioned, myocardial infarction and other cardiovascular diseases. In addition, this imaging modality might be useful in the evaluation of cell death blocking drugs, such as caspase inhibitors, for use in a clinical situation. 


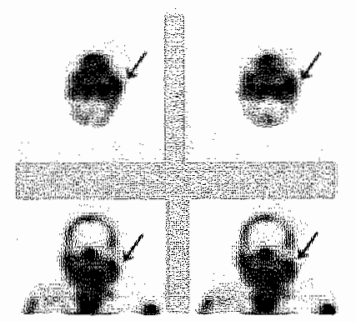

A

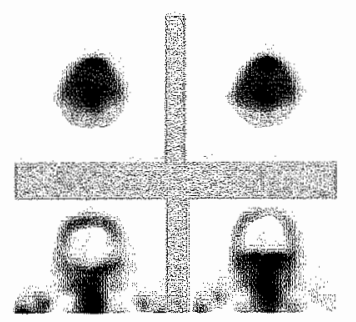

C

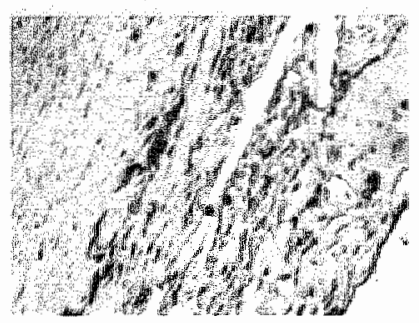

B

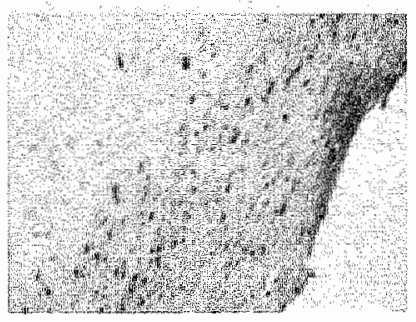

D

Figure 1.9: Annexin A5 imaging in patients with artherosclerosis. In panel A transverse and cotonal SPECT views are shown from a patient who had a transcient ischemic attack three days before imaging. Although stenosis was clinically significant in both carotid arteries, uptake of annexin A5 was only evident in the culprit lesion. Histoparhological examination (Panel B) of the tissues from this patient shows extensive binding of annexin A5 detected by rabbir anti annexin antibodies. In another patient (Panel $C$ ), who had a transient ischemic attack three months before imaging, the images do not show any uptake of annexin. $\mathrm{A}$. Similarly, the histopathological analysis did not show significant binding of annexin $A 5$, although al lesion within the carotis is evident ${ }^{121}$. Adapted from ${ }^{221}$.

\subsubsection{Cancer}

Homeostasis is also disturbed in cancer. Tumor development is caused either by increased cell proliferation or decreased cell death or a combination of both. On the contrary, cell death is increased. In some cancers, but apparently not sufficient enough to prevent the growth of the tumor. "Tc-annexin A5 is suitable for imaging the status of PS-expression within a tumor. 


\subsubsection{Intracardial tumors}

During the last few years, we have gained some experience in the field of intracardiac tumors. Clinical annexin A5 imaging in tumors was done in two rare cases of endocardial tumors. Endocardial tumors have an incidence of 0.02 to $0.3 \%$, and most endocardial tumors, about $70-90 \%$, are benign ${ }^{71.122-124}$. However, they do pose a difficult diagnostic problem for the clinician. "Classic" imaging modalities such as computer tomography (CT), magnetic resonance imaging (MRI) and echocardiography provide excellent data on localization, size, shape, hemodynamic consequences and pericardial ingrowth of the tumor. These techniques are, however, unable to inform the clinician about the benign of malignant nature of the tumor. Taking a biopsy could aid in making the diagnosis in these tumors, but this carries a very high risk of embolic complications. It is a well-known fact that in some malignant tumors, such as sarcomas, high proliferation and cell death rates are found, in sharp contrast to benign tumors. We tested the hypothesis of imaging these tumots with labeled annexin A5 to differentiate between benign and malignant tumors in the heart. For orientation purposes, a dual isotope imaging technique was used. ${ }^{201} \mathrm{TI}$ and ${ }^{99} \mathrm{~m} \mathrm{Tc}$ annexin $\mathrm{A} 5$ were infused, and imaged simultaneously. Combining the images of both compounds allowed us to localize the possible annexin A5 uptake within the area of the left ventricle. The first case was a patient presenting with collapse and progressive dyspnea. An echocardiographic image of the tumor is shown in Figure 1.10. The dual isotope imaging technique showed marked uptake of the labeled annexin. A5 within the area of the tumor (Figure 1.11). After surgery, the tumor was placed ex-vivo under the gamma camera, showing again increased radioactive uptake. Histologically the tumor turned out to be an undifferentiated sarcoma. Immunohistochemistry showed binding of annexin $A 5$ to the membranes of tumor cells, which had activated caspase 3 (Figure 1.12) ${ }^{67}$. This case report provides evidence that programmed cell death is likely to be detected in a malignant tumor by imaging with radiolabeled annexin A5. In the second case, a patient presented with similar symptoms. Again, a dual isotope imaging rechnique was used, which showed no uptake of labeled annexin A5 within the area of the tumor. The tumor ex vivo also showed hardly any radioactive uptake when placed under the gamma camera. Histology revealed a myxoma, a benign intracardiac tumor. No annexin A5 or activated caspase 3 was detected in tumor tissue. These data are shown in figure 1.13. Taken together, these cases show that the annexin A5 imaging protocol might be useful for studying the biology of tumors that are difficult to access for biopsy. 


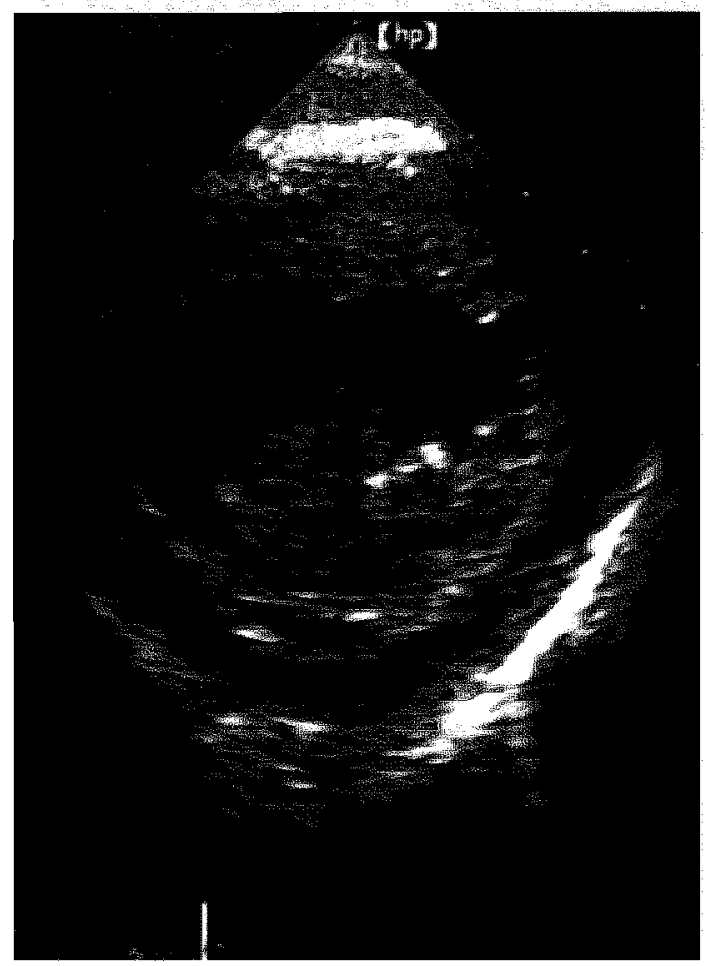

Figure 1.10: Echocardiograpic image of a malignant intracardial cumor. Adapted from ${ }^{67}$. 


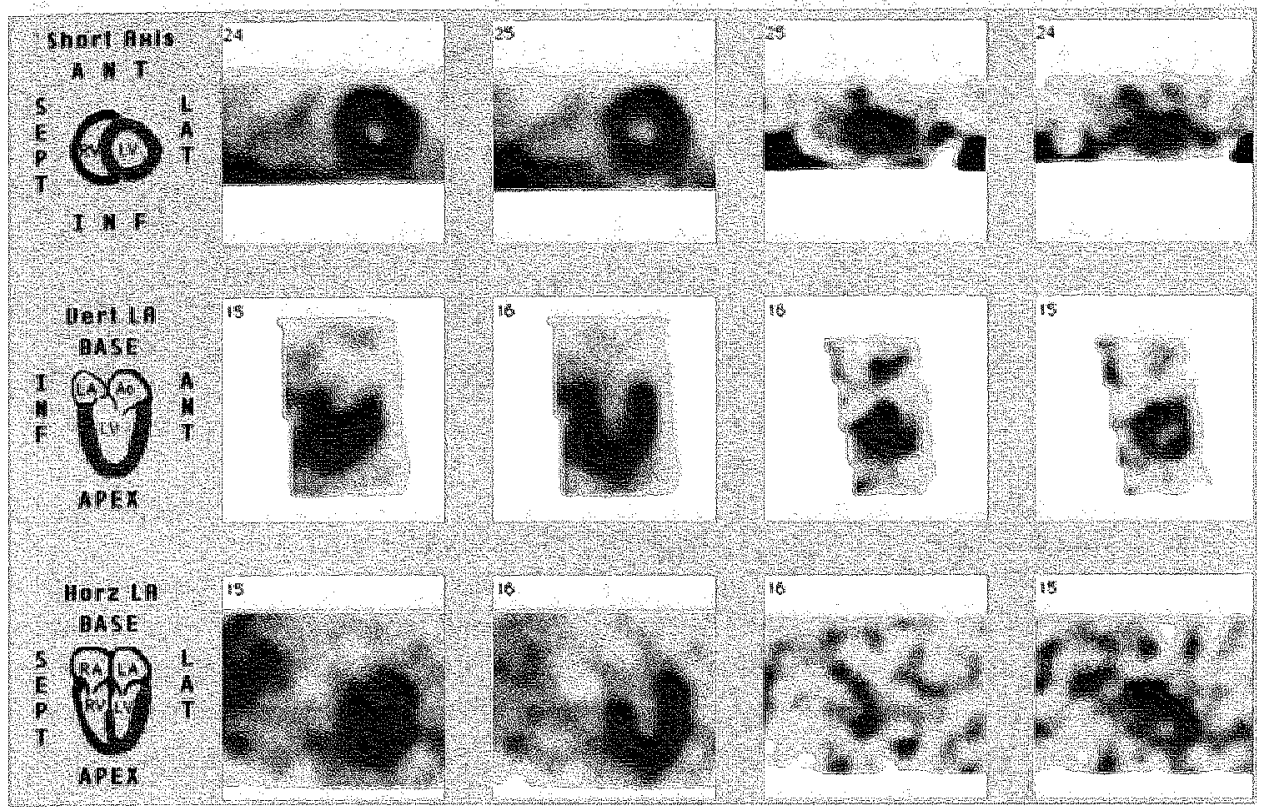

Figure 1.11: Thallium perfusion scintigraphy of a maligant tumot shows the perfused myocardium of the left ventricle in a short-axis orientation (left panel). Increased uptake of BTAP-annexin A5 can be seen within the contour of the left ventricle on short-axis SPECT, using a similar orientation as the perfusion scintigram (right panel). Adapted from ${ }^{6 \pi}$.
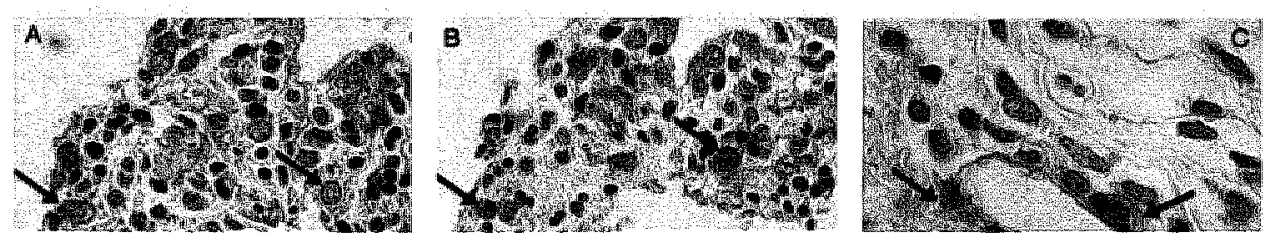

Figure 1.12: Malignant intracardiac sarcoma (immunohistology) $A$ : annexin $A 5$ staining $B$ : CM-1 antibody staining, indicating caspase 3 activation. C: Double staining with both CM1 (red) and annexin A5 antibody staining. Adapted from ${ }^{6}$. 


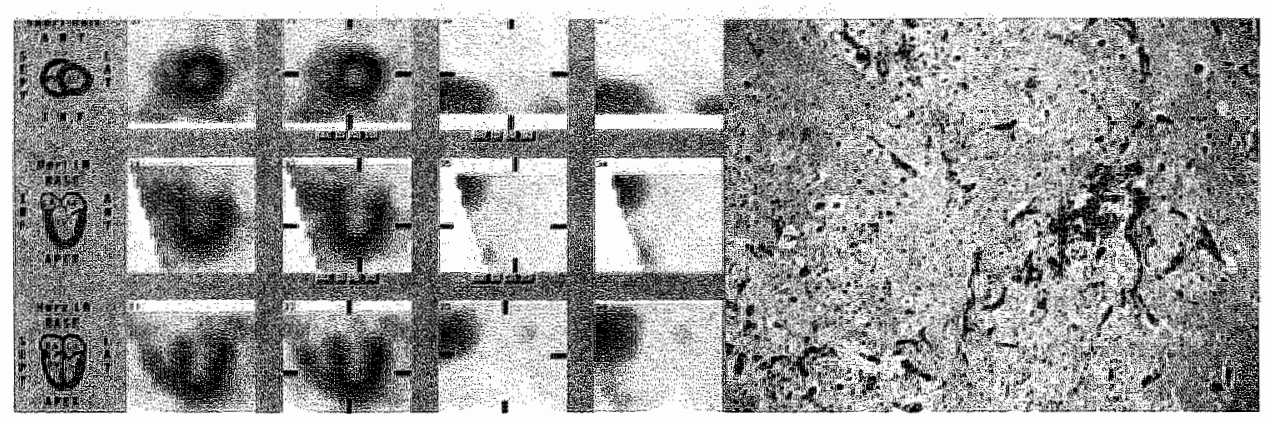

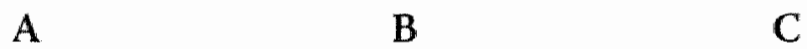

Figure 1.13. Thallium perfusion scintigraphy of a benign tumor shows the perfused. myocardium of the left ventricle in a short-axis orientation (A). Here, no increased uptake of BTAP annexin A5 can be seen within the contour of the left ventricle on short-axis SPECT, using a similar otientation as the perfusion scintigram (B). Upon histological examination, it is demonstrated that the tissue has the features of a myxoma, with clustering of nuclei and a large cellular matrices. No significant annexin A5 antibody uptake and no caspase 3 activation could be demonstrated (C). Adapted from ${ }^{71}$.

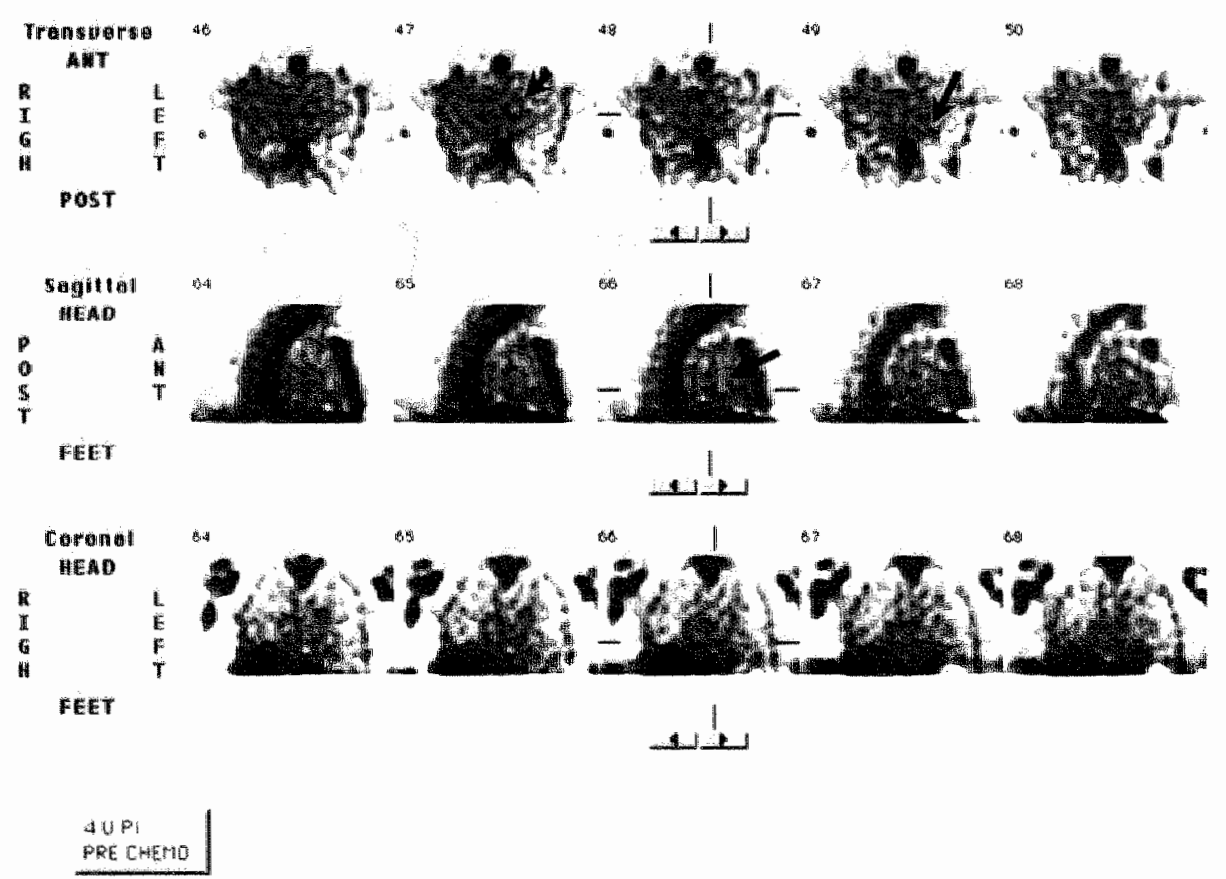

Figure 1.14: SPECT image of a non small cell lung carcinoma. Increased uptake of HYNICannexin $A 5$ can be seen within the thotax of the patient (black arrows). 


\subsubsection{Head and neck cancer}

Van de Wiele et al have succeeded in imaging programmed cell death in head and neck cancer. This study reports on the relationship between quantitative HYNICannexin A5 tumor uptake, and the number of tumor apoptotic cells derived from histologic analysis. All patients were examined by a CT scan, HYNIC-annexin A5 SPECT imaging, and then underwent surgical resection of the suspected primary or recurrent tumor. Quantitative HYNIC annexin A5 tumor uptake correlated well with the number of apoptotic cells, determined by TUNEL staining, only when tumor samples with no or minimal amounts of necrosis were considered ${ }^{70}$.

\subsubsection{Other tumor types}

We were also able to image programmed cell death in patients suffering from sarcoma, mamma carcinoma (See Chapter 3) $)^{55}$, and lung carcinoma (An example of the latter is shown in Figure 1.14).

\subsubsection{Cancer treatment efficacy evaluation using ${ }^{99 \mathrm{~m}} \mathrm{~T} c$-annexin $\mathrm{A} 5$}

Theoretically, imaging of tumors using ${ }^{99}{ }^{10} \mathrm{~T}$-annexin $\mathrm{A} 5$ has even more practical applications than in cardiac disease, because it is possible to evaluate the apoptotic status of the tumor before and after therapy. The animal work of Mochizuki ${ }^{102}$ shows that effectiveness of chemotherapy can be evaluated after a single dose of chemotherapy. Currently there is some evidence that the same principle can be applied in clinical medicine. If this evidence is sustained by large clinical trials, this would be an extremely valuable addition to conventional imaging techniques, because this could lead to more accurate, individualized therapy, and ultimately better survival. This may influence clinical decision making, and aid in timing surgery or guide the use of chemotherapy. The first attempt in the direction of chemotherapy evaluation was made by Belhocine and colleagues. They showed that responders who had a positive $99 m$ Tc-annexin A5 scan after chemotherapy, had a better survival rate than those who did not. In all cases, no tracer uptake was observed before treatment. Seven patients showed ${ }^{99 m}$ Tcannexin A5 uptake at tumor sites $24-48$ h after the first course of chemotherapy. These patients had either a complete $(n=4)$ or a partial response $(\mathrm{n}=3)$. Furthermore, 6 of the 8 patients who showed no significant post treatment tumor uptake of ${ }^{99 \mathrm{~m} T}$ T-annexin A5 had progressive disease. Two parients with breast cancer had a partial response, despite the lack of tracer uptake after treatment. Overall survival and progression-free survival were related to tracer uptake in treated lung cancers and lymphomas $(\mathrm{P}<0.05)^{125}$.

Recently, Haas et al have shown that the principle of therapy evaluation can also be applied for HYNIC-annexin A5 in patients undergoing radiotherapy ${ }^{69}$. Eleven patients with follicular lymphoma and recurrent disease underwent ${ }^{9 y m}$ 'T $c$-annexin A5 imaging before and 24 hours after the last fraction of the $2 \times 2$ Gy involved field 
radiotherapy regimen. Cytology was performed to determine the optimal time window for apoptosis detection and to confirm the apoptotic nature of the response. The ${ }^{99 m}$ Tc-annexin A5 scintigraphy (total body studies and SPECT of the irradiated sites) was performed 4 hours p.i. Tumor uptake was scored in a semiquantitative manner. Response evaluation was performed after 1 week and 4 weeks both in terms of completeness and speed of remission. Baseline ${ }^{99 \mathrm{~m}} \mathrm{~T} C$-annexin $\mathrm{A} 5$ uptake was absent in 6 and weak in 5 patients. Sequential cytology indicated that the optimal time period for apoptosis assessment was between 24 and 48 hours after the last fraction of the $2 \times 2$ Gy regimen. Baseline cytology correlated with baseline ${ }^{99} \mathrm{gn} T \mathrm{~T}$-annexin A5 in all patients. Apoptotic histology features after low-dose irradiation matched the irradiation response in all patients. In all except one patient the posttreatment $99 \mathrm{~m}$ Tc-annexin A5 uptake matched the post treatment cytology. In these 10 patients the cytology and ${ }^{99_{\mathrm{m}} \mathrm{T}} \mathrm{T}$-annexin A5 results correlated well with the clinical response. Therefore, the authors conclude that tumor ${ }^{99 \mathrm{~m}} \mathrm{Tc}-\mathrm{annexin}$ A5 uptake is likely to increase after $2 \times 2$ Gy involved feld radiotherapy. This increase was concordant with the appearance of apoptotic morphology as determined by cytology, and correlated with the clinical outcome. Hence apoptotic cell death can be observed on Day 4 of this regimen and if so predicts a complete remission within 1 week ${ }^{69}$.

Furthermore, the same research group was able to identify the ${ }^{99 m} \mathrm{Tc}$-annexin A5 tumor uptake and relate these to treatment response. This study was performed in patients suffering from malignant lymphoma, leukemia, non-small cell lung carcinoma and head and neck squamous cell carcinoma. A statistically significant correlation was found between changes in ${ }^{99 m} \mathrm{Tc}$-annexin A5 tumor uptake and clinical response. Complete or partial tumor response was correlated with a marked increase of $99 \mathrm{~m}$ ' $\mathrm{TC}$ annexin $\mathrm{A} 5$ accumulation during early treatment compared to baseline values. In cases of stable or progressive disease, pretreatment scans demonstrated predominantly low 994"T Tcannexin A5 tumor uptake and no significant increase early after treatment ${ }^{126}$. We imaged patients with non-Hodgkin's lymphoma, non-small cell lung cancer and advanced breast carcinoma before and after treatment. The results were inconclusive so that further investigations are necessary.

Cancer is already an interesting disease for both tumor imaging and therapy evaluation using labeled annexin A5. However, there is a lot of information to gain on exact times berween tumor therapy and tumor imaging. Furthermore, the latter is likely to depend on the tumor characteristics as well as the kind of therapy used. 


\subsection{Possible clinical pitfall: annexin A5 antibodies}

Neutralizing antibodies against a protein-based pharmaceutical are defined as those antibodies that interfere with its biochemical function. Neutralizing antibodies or other factors that interfere with annexin binding to PS, if present in the circulation of patients studied with ${ }^{9 \mathrm{~m}} \mathrm{Tc}$-annexin A5, could potentially result in incorrect diagnoses. Administration of $99 \mathrm{~m} \mathrm{Tc}$-annexin $\mathrm{A} 5$ to a patient is theoretically able to induce the generation of antibodies, although antibody induction is uncommon for proteins administered IV. Therefore, regulatory authorities (FDA, EMEA) require testing for neutralizing antibodies for protein based pharmaceuticals including recombinant human proteins.

We developed an assay to meet this requirement. We named this assay the AnnexNeutral assay. The ApoCorrect assay ${ }^{20}$ (see chapter 2) could be adapted and used for the detection of anti-annexin A5 neutralizing antibodies or other factors that block annexin binding to PS. When FITC-annexin A5 is incubated in presence of PS-containing beads with antibody containing plasma, its bead bound fluorescence will decrease (Figure 1.15). We validated the assay using the plasma of ${ }^{99 \mathrm{~m}^{\prime \prime}} \mathrm{T} \mathrm{c}$-annexin A5-naive healthy volunteers $(n=28)$ as negative controls. As positive controls we used WAC2a-monoclonal antibody and polyclonall rabbit antibodies against annexin A5. Figure 1.15 illustrates that increasing antibody concentration causes a decrease of FITC-annexin A5 bound to the beads.

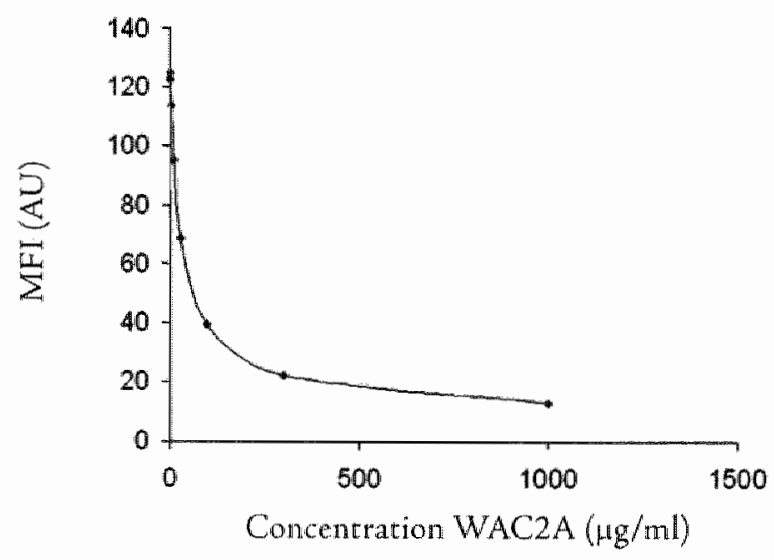

Figure 1.15: The concentration of the annexin $\mathrm{A} 5$ complexing antibody $\mathrm{WAC}-2 \mathrm{a}$ versus FITC-annexin A5 fluorescence. Binding was measured by flow cytometry and expressed as $\%$ of maximal Mean fluorescence intensity ( $\%$ MFI). 


\subsection{Future}

The perspectives of the application of annexin A5 in diagnostic techniques, treatment evaluation and therapy approaches in the future are, in our opinion, broad. The following requirements need to be met to ensure further development of annexin A5 as a diagnostic and therapeutic tool:

* The development for human use of new PET-annexin A5 probes in order to enhance the resolution of the image. Good candidates are ${ }^{68} \mathrm{Ga}$ and ${ }^{18} \mathrm{~F}$. annexin $\mathrm{A} 5$ conjugates ${ }^{85}$. As ${ }^{68} \mathrm{Ga}$ is not expensive and available through a generatot, the cyclotron-produced ${ }^{18} \mathrm{~F}$ might be not as practicable as ${ }^{68} \mathrm{Ga}$.

+ As annexin A5's biodistribution profile is not oprimal, the development of conjugates with a better biodistribution profile should be continued.

- In order to be able to perform therapy evaluation using annexin A5, a lot of information still needs to be gained in order to validate the use of annexin A5 for (anti-)apoptotic treatment of disease in humans. The latter would be of great interest for the prediction of treatment outcome in patients. 


\subsection{Conclusions}

Annexin A5 was shown to exert various biological effects in vitro. However its exact physiological function remains unknown. The ability of annexin A5 to bind PS specifically in presence of calcium is widely used to detect apoptosis. Hence, annexin A5 containing radiopharmaceuticals are an interesting development within the field of nuclear medicine. These have demonstrated the ability to visualize PS-expression in various diseases such as cancer and myocardial infarction. The use of annexin A5 can be further extended by, for instance, the development of new pharmaceuticals, which can target apoptoric cells more specifically using annexin A5 as a magic bullet. 


\subsection{Objectives of this thesis}

The following aspects of the utilisation of radiolabeled annexin A5 for cell death imaging were studied:

- A novel method to measure the biological activity of radiolabeled or Aluorescent annexin A5 was developed and validated (see Chapter 2).

- Pharmacokinetics and biodistribution in humans of two conjugation methods for annexin A5 (see Chapter 3 and 4).

- The influence of the used radiolabeling method on pharmacokinetics and imaging properties of two types of ${ }^{99 \mathrm{~m}} \mathrm{~T} \mathrm{C}$-annexin $\mathrm{A} 5$ in humans (see Chapter 3).

- The dosimetry of BTAP-annexin A5 (see Chapter 4).

- The inhibition of cell death by minocycline in the myocardium after ischemia and subsequent reperfusion was studied in $I / R$ models of mice and rabbits. Furthermore, the influence of treatment with minocycline on ${ }^{99 m} \mathrm{Tc}$-annexin A5 biodistribution in rabbits undergoing myocardial ischemia and subsequent reperfusion (I/R) was assessed (see Chapter 5). 



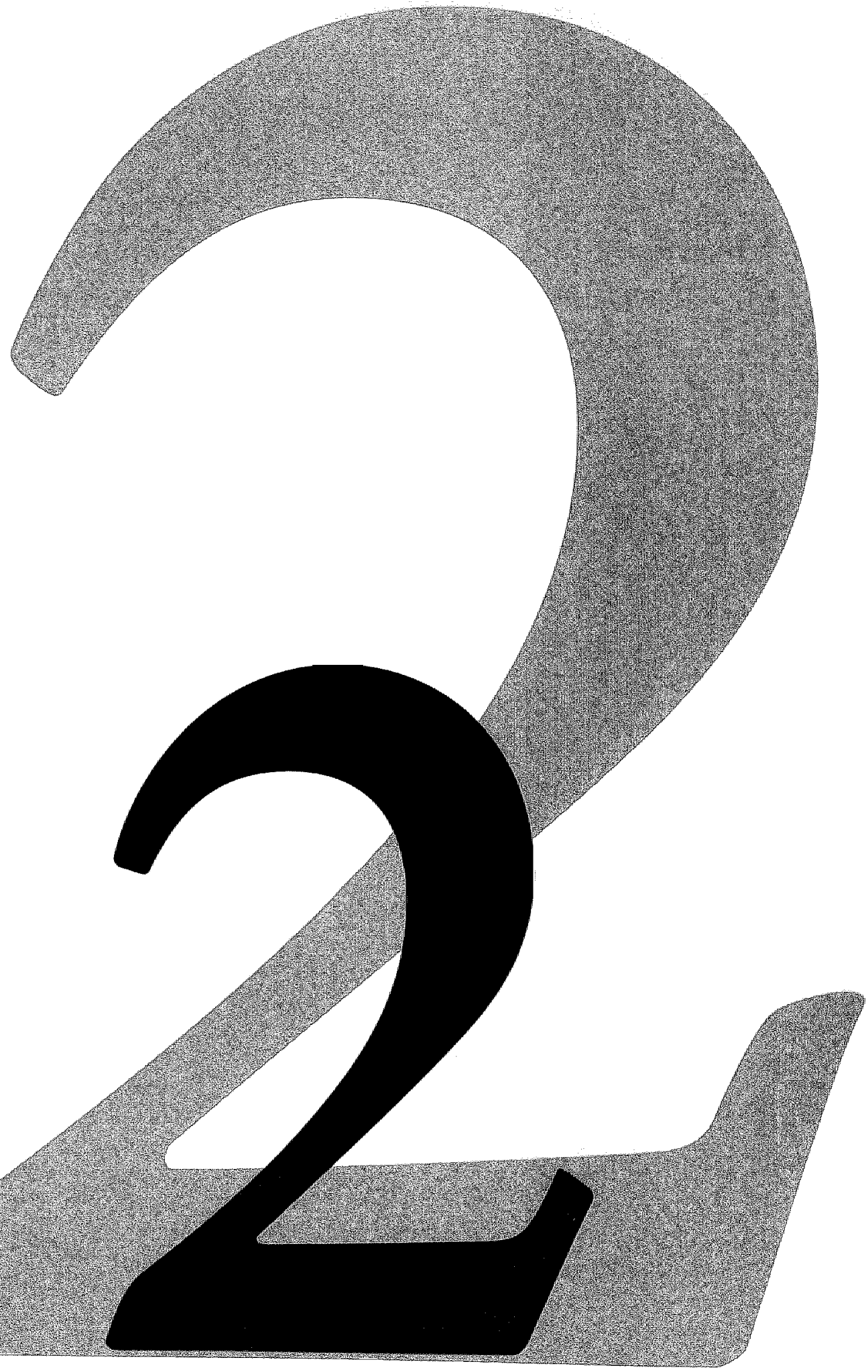





\section{The ApoCorrect ${ }^{\mathrm{TM}}$ assay $\ddagger$ a novel, rapid method to determine the biological functionality of radiolabeled and fuorescent Annexin A5}

Adapted from: H.H. Boersma, L.M.L. Stolk, H. Kenis, N.M. Deckers, J-L Vanderheyden, L. Hofstra, G.A.K. Heidendal, C.P.M. Reutelingsperger. The ApoCorrect ${ }^{\text {TM }}$ assay" a novel, rapid wethod to determine the biological functionality of radiolabeled and fuorescent Anwexin AS. Analytical Biochemistry 2004:327:126-34 


\subsection{Introduction}

Programmed cell death plays an important role both in physiology and in pathology $y^{24}$. Derailment of PCD is believed to be part of the etiology and parhology of cardiovascular diseases ${ }^{127}$, neurodegeneration ${ }^{128}$ and cancer ${ }^{129}$. Instead of being a cause of disease, PCD may also be a target for the treatment of diseases in the clinic. For instance, many anti-cancer therapies such as chemo- and radiotherapy are effective because they induce PCD of the tumor cells. Hence, the measurement of $\mathrm{PCD}$ in vivo in patients can provide information about both the pathology and the efficacy of therapy ${ }^{129}$.

Recently, a procedure was developed to measure PCD in patients with radionuclide imaging. This procedure is based on the finding that cells executing PCD expose PS at their surface ${ }^{59}$. annexin $\mathrm{A} 5$, a $36 \mathrm{kD}$ a protein ${ }^{1}$, is able to bind specifically to phospholipids such as $\mathrm{PS}$ in presence of $\mathrm{Ca}^{2+26,130,131}$. This binding is reversible. The above mentioned procedure of radionuclide imaging of $\mathrm{PCD}$ in vivo employs radiolabeled annexin A5. After validation in animal models ${ }^{65,132}$, the method was successfully applied in patients. We and others used ( ${ }^{99 \mathrm{~m}}$ Tc) -labeled annexin A5 to perform planar and SPECT imaging for the detection of PCD in myocardial infarction, several tumor types and allograft rejection after heart transplantation ${ }^{55,66,67,72}$.

The clinical procedure involves the radiolabeling of annexin A5 prior to injection into the patient. Most of the clinically applicable radionuclides have a short half-life time such as ${ }^{99 m} \mathrm{Tc}\left(t_{1 / 2}= \pm 6 \mathrm{~h}\right),{ }^{18} \mathrm{~F}\left(t_{1 / 2}= \pm 1.8 \mathrm{~h}\right)$ and ${ }^{11} \mathrm{C}\left(\mathbf{r}_{1 / 2}= \pm 20 \mathrm{~min}\right)$. These short half-life times set a narrow time-window (i.e. about $30 \mathrm{~min}$ ) between radiolabeling and injection into the patient for analysis of the radiolabeled annexin A5. To date it is general practice to analyze only the labeling efficiency of the procedure in terms of $\%$ of radiolabel associated with annexin A5. The results of this physical analysis do not provide any information about the biological functionality, and consequently, the PCD imaging capacity of the radiolabeled annexin A5. Hence, there is a great need for a rapid and simple method to determine the biological activity of radiolabeled annexin A5, which is prepared for clinical use.

This paper describes the development and initial validation of a rapid and simple method to measure the percentage of biologically active ${ }^{99 m} \mathrm{Tc}-$ annexin $\mathrm{A} 5$ within the short time-window between preparation and injection of the radiopharmaceutical. The original idea of the assay was the goal to obtain a PS surface to which the fluorescently and radioactively labeled annexin $A 5$ will bind in order to be able to separate the functionally active annexin A5 from the biologically inactive forms and other impurities like the free radioisotope used for labeling of the protein. This was achieved by coating iron oxide beads with a mixture of phospholipids, which also contains PS.

We hypothesized that, by measuring binding of annexin A5 to the beads in the presence and in the absence of $\mathrm{Ca}^{2+}$, we should be able to determine the specific binding of annexin A5 to the PS-PC-Chol beads. 


\subsection{Materials and Methods}

\section{Reggents}

All chemicals wete obtained from Merck (Darmstadt, FRG), unless otherwise specified. The phospholipids 1,2-dioleoyl-SN-glycero-3-phosphocholine (Phospharidyl choline, PC) and 1,2-dioleoyl-SN-glycero-3-[phospho-L-serine] (Phosphatidyl serine, PS) were from Avanti Polar-lipids, Inc. Anti-Fas (anti-CD95, 7C11 clone) was from Beckman/Coulter, (Mijdrecht, the Netherlands);

M-PVA-12 Iron oxide beads (M-PVA-12 beads) were obtained from Chemagen (Baesweiler, FRG). ACD-A buffer solution (Baxter, Utrechr, the Nerherlands) contains: citric acid monohydrate $8.0 \mathrm{~g} / \mathrm{l}$, sodium citrate dihydrate $22.0 \mathrm{~g} / \mathrm{l}$, glucose monohydrate $24.5 \mathrm{~g} / \mathrm{l}$ in water for injections.

Calcium buffer: $10 \mathrm{mM}$ 4-(2-hydroxyethyl)-1-piperazineethanesulfonic acid (HEPES) (ICN Biochem, Aurora, OH, USA), 0.15 M NaCl, $5 \mathrm{mM} \mathrm{KCl,} 1 \mathrm{mM}$ $\mathrm{MgCl}_{2}, 2.5 \mathrm{mM} \mathrm{CaCl}, 1 \mathrm{mg} / \mathrm{ml}$ Bovine serum albumine (Sigma), pH 7.4.

EDTA-buffer: HEPES $25 \mathrm{mM}, 140 \mathrm{mMNaCl}, 1 \mathrm{mM}$ EDTA, $1 \mathrm{mg} / \mathrm{ml}$ Bovine serum albumine (Sigma), $\mathrm{pH}=7.4$. EGTA-buffer: HEPES $25 \mathrm{mM}, 140 \mathrm{mM} \mathrm{NaCl}, 1 \mathrm{mM}$ EGTA, $1 \mathrm{mg} / \mathrm{ml}$ Bovine serum albumine (Sigma), $\mathrm{pH}=7.4$. Human recombinant Annexin A5 conjugated with hydrazinonicotinamide (HYNIC) (clinical grade) and lyophilized Stannous tricine ( $700 \mu \mathrm{g} \mathrm{Sn} \mathrm{Cl}, 2 \mathrm{H}_{2} \mathrm{O}, 21 \mathrm{mg}$ Tricine) were obtained from Theseus Imaging corporation (Boston, MA, USA).

Lyophilized stannous tricine was reconstituted with $1 \mathrm{ml} \mathrm{NaCl} 0.9 \%$ solution, prior to preparation of the radiopharmaceutical.

Human recombinant annexin A5, conjugated with Fluorescein Isothiocyanate (FITC) was provided by Nexins Research ( $\mathbb{K}$ attendijke, "The Netherlands).

The annexin A5 mutant M1234, which does not bind to PS, was prepared as described by Mira et $\mathrm{al}^{32}$, and subsequently conjugated with FITC.

For determination of the pH-effect on the stability of HYNIC-annexin A5 the following buffers were used: For pH 3.0, 4.0, and 5.0; solutions containing $25 \mathrm{mM}$ $\mathrm{Na}$-Acetate and $2.5 \mathrm{mM} \mathrm{CaCl}, 2 \mathrm{H}_{2} \mathrm{O}$ were adjusted to the respective $\mathrm{pH}$-values. For $\mathrm{pH}$ 6.0 and 7.0: solutions containing $25 \mathrm{mM} \mathrm{HEPES,} 140 \mathrm{mM} \mathrm{NaCl}$, and $2.5 \mathrm{mM}$ $\mathrm{CaCl}_{2} \cdot 2 \mathrm{H}_{2} \mathrm{O}$ were adjusted to the respective $\mathrm{pH}$-values.

For pH 8.0 and 9.0 : solutions containing $25 \mathrm{mM}$ Tris $140 \mathrm{mM} \mathrm{NaCl}$, and $2.5 \mathrm{mM}$ $\mathrm{CaCl}_{2} \cdot 2 \mathrm{H}_{2} \mathrm{O}$ were adjusted to the respective $\mathrm{pH}$-values. For all controls, similar buffers were used except that they were Calcium free and $1 \mathrm{mM}$ EDTA was added to all buffers. 
Preparation of PS-PC-Chol beads and PC-Chol beads

M-PVA-12 beads were coated with PS-PC-Chol or PC-Chol as follows:

$5 \mathrm{mg}$ of M-PVA-12 beads were washed with EDTA-buffer and suspended in $1 \mathrm{ml}$ of this buffer. All lipids were dissolved in chloroform. $1 \mu$ mole PC, $1 \mu$ mole PS and 1 $\mu$ mole cholesterol, or $2 \mu$ moles PC and $1 \mu$ mole cholesterol were added to a glass tube. The chloroform was evaporated under nitrogen gas flow giving a dried lipid film on the glass wall. $1 \mathrm{ml}$ of the washed beads was added to the lipid film and the mixture was vortexed vigorously, and subsequently submitted to ultrasonication on ice during 1 minute. The unbound lipids were removed by extensive washing with EDTA buffer. The lipid coated beads were resuspended in EDTA-buffer and stored at $4^{\circ} \mathrm{C}$.

\section{Preparation of HYNIC-annexin A5}

For each experiment, a vial containing $250 \mu \mathrm{g}$ of human recombinant annexin $\mathrm{A} 5$ conjugated with $\mathrm{HYNIC}^{65}$ was thawed after storage (at $-20^{\circ} \mathrm{C}$ ) and mixed with ca. 1.1. $\mathrm{GBq}$ pertechnetate (diluted to $1 \mathrm{ml}$ ). Specific activity of the used pertechnetate solution was $3.5 \pm 0.8 \mathrm{GBq} / \mathrm{ml}$. Subsequently, $0.1 \mathrm{ml}$ stannous tricine buffer was added to the vial for pertechnetate reduction. This mixture was allowed to incubate $(30 \mathrm{~min}, \mathrm{r.t}$.) The $\mathrm{pH}$ was measured (limits $\mathrm{pH}$ 5-7) and radiochemical purity (RCP) was assessed by TLC on silica impregnated paper (Gelman Sciences, Ann Arbor, MI) using ACD buffer as the eluent ${ }^{89}$. HYNIC-annexin A5 remains at the position of application $(\mathrm{R} f=0)$, whereas most radioactive impurities are at the solvent front $(\mathrm{R} f=\mathbb{1})$.

Induction of apoptosis in Jurkat cells and measurement of PS-expression using HYNICannexin A5 and FITC-annexin A5 (positive control)

Jurkat cells (ATCC, Rockville, MD, USA) were grown in RPMI 1640 medium (Life Technologies) supplemented with $10 \%$ heat inactivated $\left(30 \mathrm{~min}, 56^{\circ} \mathrm{C}\right.$ ) feral bovine serum (Gibco). Viability was determined by trypan blue exclusion and exceeded $95 \%$. Apoptosis induction was achieved by incubating $4 \times 10^{6}$ cells $/ \mathrm{ml}$ with $20 \mathrm{ng} / \mathrm{ml}$ Anti-fas in Medium 199 (Life Technologies) supplied with $0.5 \mathrm{mM}$ Calcium chloride for 3 hours.

HYNIC-annexin A5 binding to apoptotic cells was assessed by incubating $10 \mu \mathrm{l}$ of HYNIC-annexin A5 with $1 \mathrm{ml}$ of apoptotic Jurkat cells during 15 min at r.t.. Cells were then removed by centrifugation ( $30 \mathrm{~min}, 1000 \mathrm{~g}, \mathrm{r.t}$.). Cell-free supernatant and cell pellet were counted on radioactivity to determine HYNIC-annexin A5 bound to the cells.

FITC-annexin A5 and FITC-M1234 binding to apoptotic cells was assessed by incubating a range $0-10 \mathrm{ng} / \mathrm{ml}$ of FITC-annexin A5 or FITC-M1234 with $50 \times 10^{3}$ of apoptotic Jurkat cells during $5 \mathrm{~min}$ at r.t.. Flow cytometry was performed to measure the binding of FITC-annexin A5 or FITC-M1234 to the apoptotic cells. The same experiments were performed in the presence of EDTA and EGTA. 
Flow cytometric analysis of the beads, phase contrast microscopy and furerescent microscopy

FITC-annexin A5 was incubated with $4 \mu \mathrm{g} / \mathrm{ml}$. phospholipid beads in Calcium buffer during 30 minutes at r.t. The amount of FITC-annexin A5 per bead was measured using flow cytometry. The binding of FITC-annexin A5 to the beads was inspected visually with fluorescence microscopy. The shape of the beads was inspected using phase contrast microscopy. The competition between HYNIC-annexin A5 and FITC-annexin A5 was assessed by incubating mixtures of these annexins with $4 \mu \mathrm{g} /$ $\mathrm{ml}$ phospholipid beads in Calcium buffers during 30 minutes at r.t. Competition of unmodified wild type annexin $A 5$ with FITC-annexin $A 5$ served as a control. The amount of FITC-annexin A5 bound was then measured by flow cytometry.

\section{ApoCorrect ${ }^{\mathrm{TM}}$ assay}

Phospholipid beads (ca. $17 \mathrm{mg} / \mathrm{ml}$ ) in $180 \mu \mathrm{l}$ Calcium buffer were incubated in a microfuge vial of $1.5 \mathrm{ml}(15 \mathrm{~min}$, r.t.) with $10 \mu \mathrm{l}$ of the HYNIC-annexin A5solution. Using a magnetic device, the beads were kept in situ when all supernatant was removed. Radioactivity of supernatant and beads was measured separately. The results of the two procedures are applied in the following equation to give the fraction of biologically active HYNIC-annexin A5:

$\%$ biologically active ${ }^{99}$ Tc-annexin A5:
$\left(\mathrm{B}_{\mathrm{C}_{2}{ }^{2}+\mathrm{x}} 100 \%\right)$
(B $\left.{ }_{\text {Cheitaror }} \times 100 \%\right)$
$\left(\mathrm{B}_{\mathrm{Ca}^{2+}}+\mathrm{SCa}^{2+}\right)$
$\left(\mathrm{B}_{\text {Chelator }}+\mathrm{S}_{\text {Chelarour }}\right)$

$\mathrm{B}_{\mathrm{Ca}^{2+}}=$ amount of radioactivity, bound to the beads in binding buffer.

$\mathrm{S}_{\mathrm{C}^{2}}{ }^{2+}=$ amount of radioactivity, in supernatant (binding buffer).

$\mathrm{B}_{\text {Chelaror }}=$ amount of radioactivity, bound to the beads in EDTA or EGTA buffer.

$\mathrm{S}_{\text {Chelarer }}=$ a mount of radioactivity, in supernatant (EDTA or EGTA buffer). 
Repeatability, reproducibility and stability indicating properties of the ApoCorrect ${ }^{\mathrm{TM}}$ assay In order to determine repeatability the ApoCorrect ${ }^{\mathrm{TM}}$ assay $(n=6)$ was performed on one day. To measure reproducibility, the assay was done six times on different days. Repeatability and reproducibility were obtained by calculating the respective intra- and interassay coefficients of variation. For these experiments, one single lot of HYNIC-annexin A5 was used.

Stability indicating properties were also determined. Firstly two lots of different age were compared. Secondly, the effects of $\mathrm{pH}\left(3\right.$ through 9) and temperature $\left(60^{\circ} \mathrm{C}\right)$ on the stability of HYNIC-annexin A5 were measured. Under all circumstances, controls with EDTA buffer were performed in order to determine nonspecific binding to the beads. Also, controls with EGTA buffer and PC-Chol beads were used for further validation of the assay.

Statistical evaluation

Data were statistically evaluated using Student's t-test. A probability value $<0.05$ was considered significant. Results are generally presented as mean \pm standard deviation. 


\subsection{Results and Discussion}

Annexin A5 is a molecular imaging agent used to visualize apoptosis in vitto and in vivo in animal models and patients. Annexin A5 imaging of apoptosis in patients may serve as an aid to diagnosis and the evaluation of the efficacy of therapy. These prospects are currently substantiated in preclinical and clinical studies ${ }^{55,66,67,72,125}$. Biodistribution in humans and the first clinical application of HYNIC-annexin A5 were described recently ${ }^{70,90}$. HYNIC-annexin A5 can be prepared faster, is more stable and has higher yields than the other used labeling procedures (BTAP, n-1imino-mercaptobutyl) ${ }^{55,90}$ (See also Chapter 1). The HYNIC conjugation method facilitates labeling of pertechnetate to primary aminogroups within the annexin A5 molecule. A pitfall of the HYNIC-annexin A5 imaging procedure remains the high uptake in kidney, liver, and spleen, thus hampering imaging of these organs. Another disadvantage is the long biological half life of the radiopharmaceurical (69 h), resulting in a rather high absorbed radioactive dose in the individuals ${ }^{90}$.

In this perspective, it is important to optimize the specificity and sensitivity of the annexin $A 5$ imaging procedure. In vivo use of annexin $A 5$ in patients involves a procedure to label annexin $A 5$ with ${ }^{99 m} \mathrm{~T} T$. Up to now determination of labeling efficiency (RCP) was the only quality control available before the labeled annexin A5 was injected into the patient. There was a lack of a rapid assay to determine the biopotency of annexin A5 after the radiolabeling. The ability of annexin A5 to bind to apoptotic cells fully depends on its ability to bind to phosphatidylserine (PS) in a $\mathrm{Ca}^{2+}$ - dependent manner ${ }^{49,100}$.

Hence, an assay that accurately measures the phospholipid binding of annexin A5 would be a proper quality control method.

In the present study, we describe the development and initial validation of a fast, simple and specific biopotency assay that can be used for quality control testing of ${ }^{99 \mathrm{~m}} \mathrm{Tc}$-annexin A5.

\section{Characterization of the binding of Annexin A5 to phospholipid coated beads}

Ourgoal was to have a rapid assay to measure the biologically active fraction of HYNIC. annexin A5. The short half life of ${ }^{99 m} \mathrm{Tc}$ and other radioisotopes such as ${ }^{18} \mathrm{~F}$ and ${ }^{68} \mathrm{Ga}$ require rapid evaluation of binding and biopotency of the radiopharmaceutical. Therefore we decided to develop a solid phase-assay on the basis of paramagnetic beads, M-PVA-12 of Chemagen, which have a size distribution between $1-3 \mu \mathrm{m}$ (for further information see www.chemagen.de/de_uk/uk/firstset_fearures.html). The beads can be visualized by light microscopy and measured by flow cycometry and separated from the solution in a magnetic field.

In order to assess the biological activity of annexin A5, we coated the beads with phospholipids containing PS. Firstly we visualized FITC-annexin A5 binding to 
the beads coated with a phospholipid mixture consisting of PC/PS/Chol (33/33/33, moles \%) using fluorescence microscopy (Figure 2.1A). Figure $2.1 \mathrm{~B}$ shows a phase contrast image, which gives an impression of the reproducible shape and size of the beads.

We prepared 6 lors of the PS-PC-Chol beads, which were equally able to bind FITCannexin $\mathrm{A} 5$ in the presence of $\mathrm{Ca}^{2+}$, examined by flow cytometry.

A
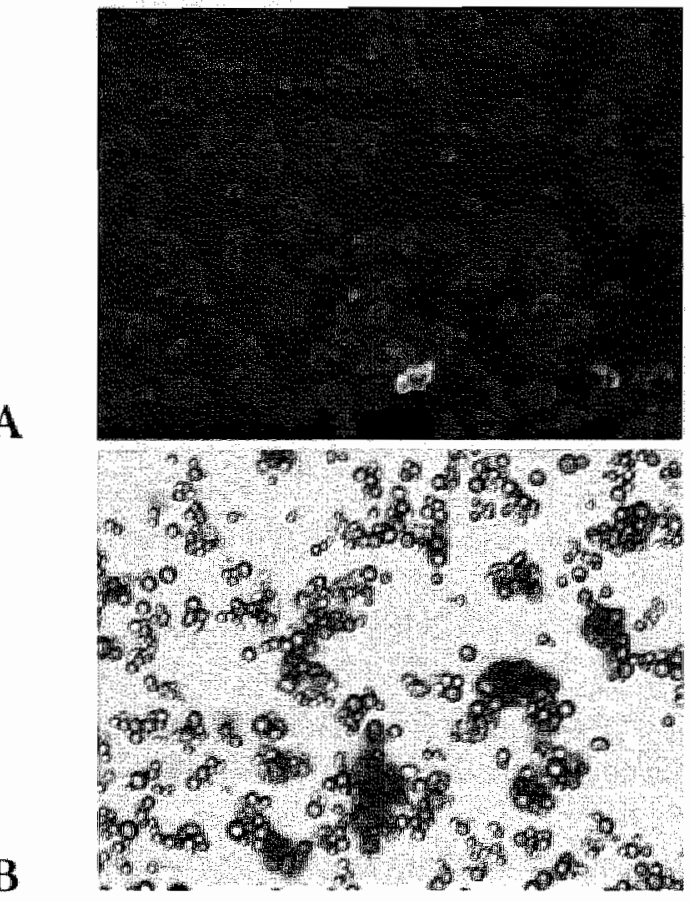

B

Figure 2.1(A): Fltorescence microscopy and 2.1 (B): Phase-contrast microscopy of PS-PCChol beads and FITC-Annexin A5 in calcium buffer.

An example of this finding is given in Figure 2.2A, which illustrates the binding of FITC-annexin A5 to the phospholipid beads. This binding depends on the presence of calcium ions and functional calcium/ phospholipid binding sites on the annexin A5 molecule since no binding is observed in the presence of EDTA or EGTA and with the non binding annexin A5 mutant FITC-M1234 ${ }^{32}$, respectively (Figure 2.2B). In addition, no binding occurs if PS is omitted in the phospholipid coat (Figure 2.2B), demonstrating the specificity of the interaction between FITC-annexin A5 and the phospholipid coated beads. 
The phospholipid coated beads bound with FITC-annexin A5 give a typical scatrer patten (Figure 2.2A) that is reproducible for the described coating procedure. Figure 2.2D illustrates dose-tesponse curves for FITC-annexin A5 concentration and Mean Fluorescence Intensity (MFI) of the phospholipid coated beads as measured by flow cytometry at different bead concentrations. Binding of annexin-FITC to the phospholipid coated beads can be measured quantitatively when expressed in MFI of the histogram (Figure 2.2D). Assuming an average bead diameter of $2 \mu \mathrm{m}$ it can be calculated that 1 bead contains abour $10^{-19}$ moles annexin $A 5$ binding sites ${ }^{26}$. Furthermore, $1 \mathrm{mg}$ of beads comprises of approximately $10^{\circ}$ beads. Hence, the doseresponse curve at $3.9 \mu \mathrm{g} / \mathrm{ml}$ beads is amenable to analysis with the Langmuir [FITC. annexin A5] versus $\mathrm{MFI}^{-1}$ plot. This analysis yields a $\mathrm{Kd}$ of less than $0.1 \mathrm{nM}$ for FITC-Annexin A5 binding to the beads which is in concordance with the reported $\mathrm{Kd}$ estimation for annexin A5 binding to a planar phospholipid bilayer ${ }^{26}$.

The binding of HYNIC-annexin A5 to the phospholipid coated beads is assessed in a comperition experiment with FITC-annexin $A 5$ and compared to the competition between unlabeled Annexin A5 and FITC-annexin A5. Figure 2.2E shows HYNICannexin $\mathrm{A} 5$ acting as a competitor similat to unlabeled annexin $\mathrm{A} 5$ demonstrating that the HYNIC-derivatization does not alter the phospholipid binding properties of annexin A5. In contrast, it was observed that using the red blood cell assay, a slight difference has been determined between HYNIC annexin A5 and the wild type ${ }^{65}$, Also, we showed that the non-binding annexin A5 mutant M1234 was not able to bind to the PS-PC-Chol beads in the presence of $\mathrm{Ca}^{2+}$, which proves that the binding is specific (Figure 2.2B). This fact was used in the further development of the assay for radiopharmaceutical purposes. 

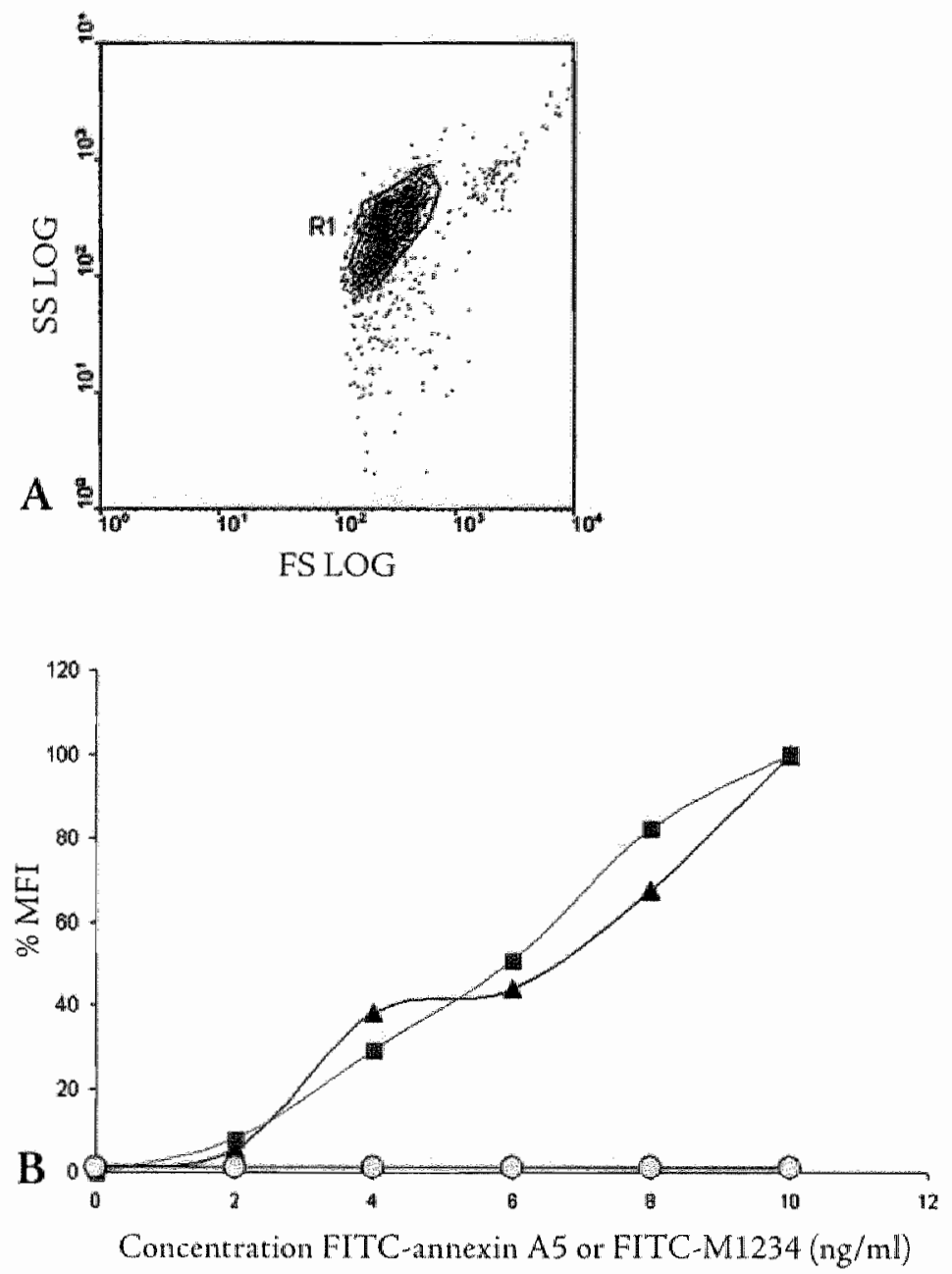

Figute 2.2A: Flow cytometry dot plot of side scarter (SS) versus forward scatter (FS) parameters of FITC-annexin A5 bound to PS-PC Chol beads. The area R1 indicates the size distribution of the beads in the presence of $\mathrm{Ca}^{2+}$. SS and $\mathrm{FS}$ are expressed in arbittary units. 2.2B: Calcium-dependent binding FITC-annexin A5 to lipid-coated beads. Concentration tesponse curves are shown for binding of FITC-Annexin A5 in the presence of $\mathrm{Ca}^{2+}$ to PS-PC-Chol beads (A), PC-Chol beads (0) and apoprotic Jurkat cells ( $)$. Binding was measured by flow cytometry and expressed as \% of maximal Mean Auorescence intensity (\% MFI). No binding occurred if FITC-M1234 was used in the presence of $\mathrm{Ca}^{2+}$ or FITC-annexin A.5 in the presence of EDTA or EGTA. 2.2C: $\mathrm{Ca}^{2+}$-dependent binding of 


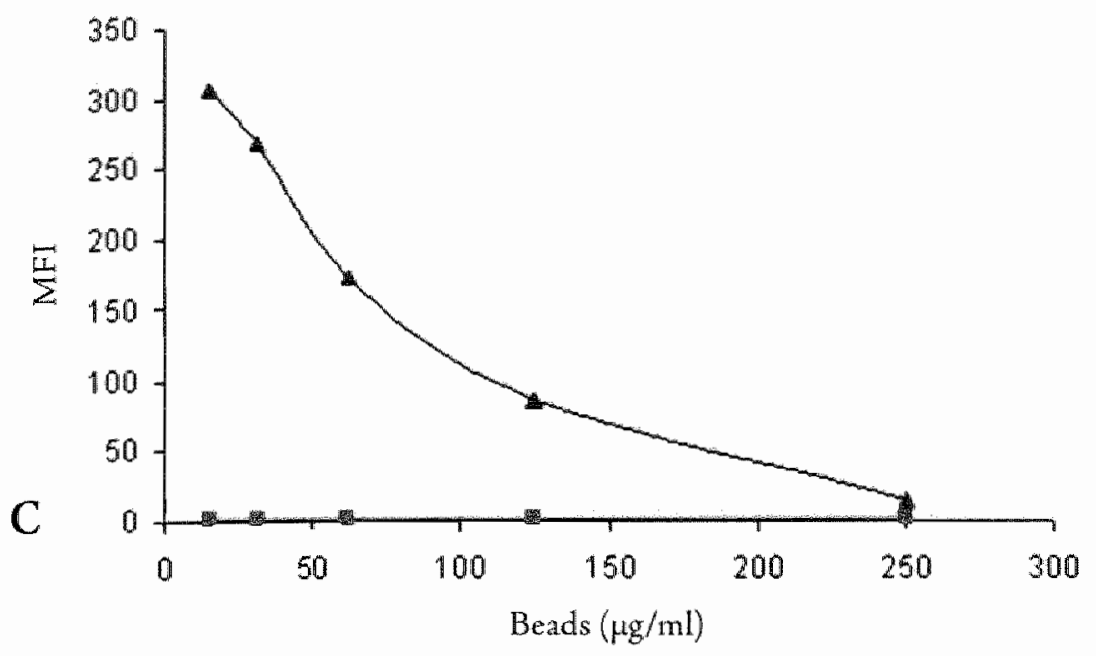

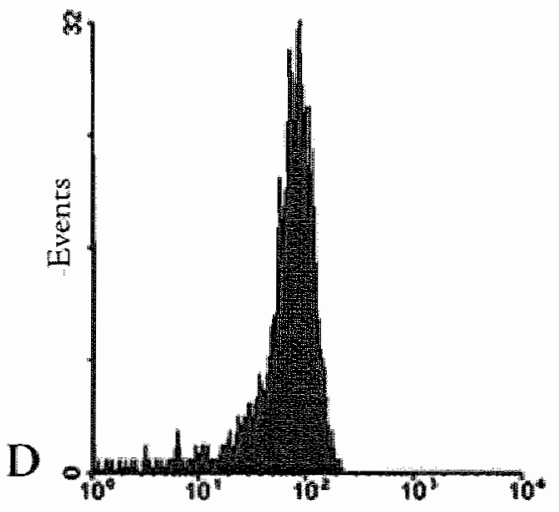

FITC-annexin A5

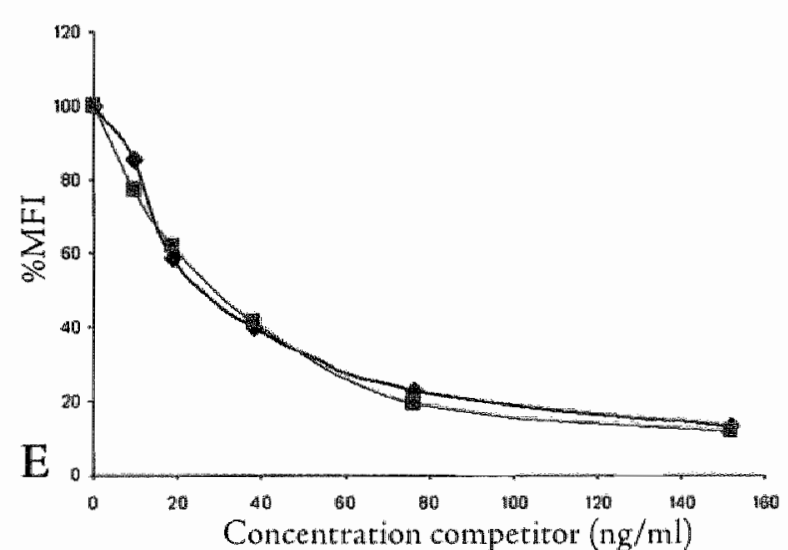

Concentration competitor $(\mathrm{ng} / \mathrm{ml})$

FITC-annexin A5 to lipid coated beads as function of the bead concentration. FITC-annexin A5 binding to PS-PC-Chol beads was measured in the presence of Ca ${ }^{2+}(\boldsymbol{\Delta})$ or EDTA ( by How cycometry. Binding was expressed as Mean Fluorescence Intensity (MFI).2.2D: Flow cyrometric histogram of FITC-annexin A5 bound to PS-PC-Chol beads in the presence of $\mathrm{Ca}^{2+}$. This is a typical histogram from which the MFI is calculated. 2.2E: Comperition of FITC-annexin A5 against wild type anmexin A5 $\downarrow$ ) and ${ }^{99}$ Tc-HYNIC-annexin A5 (1) are shown. The percentage of maximum MFI is plotted against the concentrations of the respective competirors. No difference is seen between the competitive properties of both agents. 
Initial validation of the A pocorrect $t^{\text {st }}$ assay: phospholipid coated beads as a means to determine the biologically active fraction of a ${ }^{9 \%}$ TC-HYNIC annexin A5 preparation. The analyses so far demonstrate the specificity of FITC-annexin $A 5$ binding to the phospholipid coated beads. For the analysis of HYNIC-annexin A5 we designed the Apocorrect ${ }^{\text {tw }}$ assay (see Materials and Methods section) in which the number of binding sites provided by the beads is not limiting.

The effects of $\mathrm{pH}$ and temperature on the biological active fraction of Stability indicating properties of the assay were assessed by measuring the effect of ${ }^{99 \mathrm{~m}} \mathrm{TC}$ HYNIC-annexin A5 were evaluated by exposing ${ }^{99 m} \mathrm{Tc}-\mathrm{HYNIC}$-annexin $\mathrm{A} 5$ to $\mathrm{pH}$ tanging from $3-9$ and to $60{ }^{\circ} \mathrm{C}$, respectively. The results are compiled in Figure 2.3 and Table 2.1 , respectively

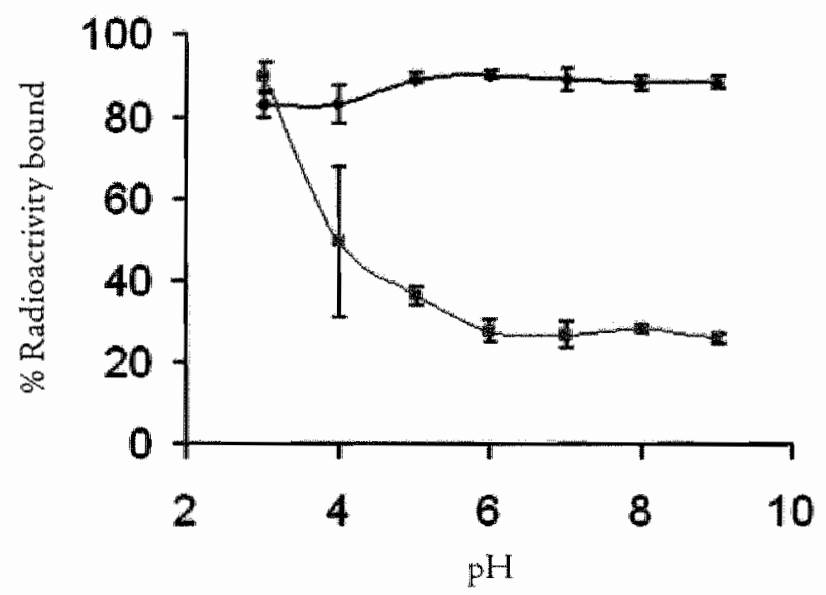

Figure 2.3: Effect of $\mathrm{pH} 3.9$ on ${ }^{99 m} \mathrm{Tc}-\mathrm{HYNIC}$-annexin A5 biopotency. Both percentages of ${ }^{99 m \mathrm{~m} T}$ TC-HYNIC-annexin A5 bound to the beads in Calcium buffer ( and in EDTA-buffer (10 are shown. It is observed that at low $\mathrm{pH}$, annexin is able to bind to the beads in absence of $\mathrm{Ca}^{2+}$ whereas this is not possible at $\mathrm{pH}>5$. All values are the results of triplicate experiments $\pm S D$. 
Table 2.1: Effect of temperature on specific ${ }^{99}$ Tc-HYNIC-annexin A5 binding to PS-PC. Chol beads using the Apocorrect -assay. Specific binding and radiochemical purity (RCP) are expressed as a percentage. All values are the mean of triplicate experiments $\pm \mathrm{SD}$.

\begin{tabular}{|c|c|c|c|}
\hline Conditions & $\begin{array}{l}\text { ApoCorrect }{ }^{\text {my }} \text {-assay, } \\
\text { specific binding }(\%) \\
\text { Mean } \pm S D\end{array}$ & $\begin{array}{l}\operatorname{RCP}(\%) \\
\text { Mean } \pm \mathrm{SD}\end{array}$ & $\begin{array}{l}\text { P-walue (ApoCorrect }{ }^{\mathrm{mu}} \text {-assay } \\
\text { compared to control) }{ }^{\mathrm{i}}\end{array}$ \\
\hline $60^{\circ} \mathrm{C}(15 \mathrm{~min})$ & $2.1 \pm 5.4 \%$ & $95.2 \pm 1.8 \%$ & $P<0.005$ \\
\hline $60^{\circ} \mathrm{C}(60 \mathrm{~min})$ & $11.2 \pm 3.5 \%$ & $94.3 \pm 1.1 \%$ & $\mathrm{P}<0.005$ \\
\hline Control $\left(\mathrm{r}_{*} \mathrm{t}_{*}\right)$ & $79.3 \pm 1.1 \%$ & $95.6 \pm 1.1 \%$ & N.A. \\
\hline
\end{tabular}

${ }^{1}$ A paired t-test was used for determination of statistical significance N.A.: not applicable; r.t.: room temperature

Table 2.2: Specific binding of HYNIC-annexin A5 to PS-PC-Chol coated beads or PCChol beads as measuzed using either EDTA or EGTA-buffer to determine the non-specific binding at room temperature. Specific binding is expressed as a percentage. The RCP of the HYNIC-annexin A5 was $92.3 \pm 2.8 \%$. All values ate the mean of triplicate experiments \pm $\mathrm{SD}$.

\begin{tabular}{|ll|}
\hline Conditions & $\begin{array}{l}\text { ApoCor recr } \\
\text { Mean } \pm \text {-assay, specific binding (\%) }\end{array}$ \\
\hline PS-PC-Chol wersus EDTA (control) & $79.2 \pm 1.3 \%$ \\
\hline PS-PC-Chol wersus EGTA & $80.3 \pm 2.0 \%$ \\
\hline PC-Chol versus EDTA & $-0.26 \pm 1.1 \%$ \\
\hline PC-Chol versus EGTA & $-0.74 \pm 3.3 \%$ \\
\hline
\end{tabular}

Measurements performed under different $\mathrm{pH}$ values revealed a statistically significant effect $(\mathrm{p}<0.05)$ between $\mathrm{pH} 3$ and $\mathrm{pH}$. Also, the binding to the beads at $\mathrm{pH} 3$ and $\mathrm{pH} 4$ did nor differ berween Calcium buffer and EDTA buffer indicaring nonspecific binding when HYNIC-annexin $\mathrm{A} 5$ is exposed to low $\mathrm{pH}$. Similar binding properties of annexin $\mathrm{A} 5$ at $\mathrm{pH} 4$ to $\mathrm{PS}$-containing vesicles in the absence of $\mathrm{Ca}^{2+1}$ were described before by Köhler et al. ${ }^{19}$. They showed that annexin $\mathrm{A} 5$ binding to PS is calcium-independent due to a $\mathrm{pH}$-dependent change in charge of the molecule. Binding characteristics are markedly changed, because the hydrophobicity largely increases at low $\mathrm{pH}$, resulting in a penetration of the phospholipid bilayer. The $\mathrm{Ca}^{2+}$ dependent binding of annexin $\mathrm{A} 5$ at physiological $\mathrm{pH}$ was shown to be of an extrinsic 
nature without penetration of the protein into the membrane ${ }^{19}$. They also described that the $\mathrm{Ca}^{2+}$ dependent binding of annexin $\mathrm{A} 5$ at $\mathrm{pH} 4$ could be restored after $10 \mathrm{~min}$ by $\mathrm{pH}$-elevation to $7.4^{19}$. After $2 \mathrm{~h}$ of incubation using HYNIC-annexin $\mathrm{A} 5$ at $\mathrm{pH} 4$, we were not able to reproduce this result. Thus, it seems likely that an incubation of $2 \mathrm{~h}$ at $\mathrm{pH} 4$ results in an irreversible change in the conformation of HYNIC-annexin A5. We observed that HYNIC-annexin $\mathrm{A} 5$ precipitated at $60^{\circ} \mathrm{C}$ within 15 min of incubation, indicating protein denaturation. The TLC results were not affected by the $60^{\circ} \mathrm{C}$ incubation showing that this method is unable to discriminate between native and denatured HYNIC-annexin A5.

To serve as a positive concrol of PS binding to HYNIC-annexin A5, apoptotic Jurkat cells were used. They were resuspended in binding buffer, and $85.6 \%$ of HYNICannexin $A 5$ was bound to the cell pellet, whereas $2.6 \%$ of HYNIC-annexin A5 was found to be bound to the cells when suspended in EDTA buffer.

Furthermore, the ApoCorrect" assay was performed with PC-Chol beads, as well as in presence of the $\mathrm{Ca}^{2+}$ specific chelator EGTA and compared with EDTA. Results of these experiments are shown in Table 2.2. It indicates that PC-Chol beads do not bind HYNIC-annexin A5. As well, the Apocorrect ${ }^{\text {mw }}$ assay was performed with HYNIC-annexin A5 using a background of EGTA, showing no significant difference with the regular assay (EDTA-background, Table 2.2).

Repeatability (Intra-assay variation), reproducibility (Inter-assay variation) and comparison of two lots of HYNIC-annexin A5

Other important criteria of a quality control assay are repeatability and reproducibility. Repeatability was performed using one single preparation on one day. Mean percentage HYNIC-annexin A5 specifically bound to the beads was $75.4 \pm 3.1$ $(n=6)$. The coefficient of variance (CV) was $4.1 \%$. Radiolabeling yielded. HYNIC. annexin A5 with a RCP of $91 \%(n=1)$, The $\mathrm{pH}$ of the obtained solution was 6.0. Reproducibility was determined using separate preparations. The mean percentage 99n"Tc-HYNIC-annexin A5 specifically bound to the beads was $69.6 \pm 7.1 \%(\mathrm{n}=6)$. The $\mathrm{CV}$ was $10.2 \%$. The RCP was found to be $89.7 \pm 1.2 \%$. The CV was $1.4 \%$. The $\mathrm{pH}$ of the obtained solutions was $6.0 \pm 0.5$.

Two lors were compared by performing the Apocorrect ${ }^{\text {ri }}$ assay. Each assay was performed in triplicate. The oldest lot (15 months after production) yielded specific binding of $73.8 \pm 1.4 \%$ and an RCP of $84.7 \pm 1.8 \%$ using TLC. For the newer lot ( 12 months after production) a specific binding of $72.4 \pm 1.4 \%$ and an RCP of $95.1 \pm 1.1$ $\%$ were obtained, respectively. Only for RCP, a significant difference between the two lots was found $(\mathrm{p}<0.05)$. This suggests that the labeling with technetium is affected. more during its shelf-life than the ability of annexin A5 to bind PS. As well, it must be stressed that the used preparation of clinical grade ${ }^{99 \mathrm{~m}} \mathrm{Tc}$-HYNIC-annexin A5 was already optimized befor ${ }^{89}$. Verbeke and co-workers show that HYNIC-annexin 
A5 conjugation results in a substantial amount of higher molecular weight aggregates ( $8.7 \%$, shown by FPLC) when one molecule HYNIC per annexin. A5 is introduced into the reaction mixture. The optimal reaction stochiometry tumed out to be 0.5 HYNIC per molecule annexin A5, yielding only $2.2 \%$ higher molecular weight aggregates. Conjugation of HYNIC to annexin A5 is likely to cause marginal loss of biological activity. The primary aminogroups to which HYNIC binds are apparently not involved in the PS binding property of annexin $A 5^{89}$. 


\subsection{Conclusion}

HYNIC-annexin A5 is under investigation to be licensed as a radiopharmaceutical for the determination of programmed cell death in vivo in patients.

In this study, a novel specific and rapid assay for ${ }^{99 m}$ Tc-labeled annexin A5 and FITCannexin $A 5$, used clinically, has been described. We show that this assay measures the biological activity of annexin A5 that underlies its apoptotic cell binding property. This in contrast with TLC, which only measures the actual binding of the isotope to the protein. We furthermore demonstrate that the ApoCorrect ${ }^{\text {m }}$ assay is robust, repeatable and reproducible to measure repeatably, reproducibly and quickly the fraction of biologically active annexin A5. Its speed (ca. 25 min per analysis) makes it suitable for use in a clinical setting. 


$$
3
$$




\section{Comparison between human pharmacokinetics and imaging properties of two conjugation methods for ${ }^{99 \mathrm{~m}} \mathrm{Tc}$-Annexin A5}

Adapted from: H.H. Boerswa, I.H. Liem, G.J. Kemerimk, P.W.L. Thimister, L. Hofstra, L.M.L. Stolk, W.L. van Heerde, M-T. W. Pakbiers, D. Janssen, A.J. Beysens, C. P.M. Reutelingsperger, G.A.K. Heidendal. Comparison between buman pharmacokinetics and imaging propertics of two conjugation methods for "99m TC-Annexinn A5. BrJ Radiol 2003; 76: 553-60. 


\subsection{Introduction}

Apoptosis or programmed cell death plays a pivotal tole not only in normal physiology but also in pathological processes. Many drugs, such as cytostatics, affect apoptosis ${ }^{132}$. Thus, the detection and quantification of apoptosis in wivo might provide insight into the disease status and/ or treatment efficacy of a patient.

The externalisation of PS at the cell membrane is one of the first events of apoptosis and persists until the cell is destroyed ${ }^{59}$. A naturally occurring protein, annexin A5, binds with high affinity to PS on apoptotic cells. For this reason, radioactively or fuorescently labelled annexin A5 has been investigated in vitro as well as in wivo in animal models as a tool to detect apoprosis ${ }^{65,81,100,133}$. Recently, our group and others demonstrated that ${ }^{99_{\mathrm{m}}}$ Tc-annexin $\mathrm{A} 5$ can be used successfully to image apoptosis in patients ${ }^{66,6772}$. In this study, two preparations of ${ }^{99 m} \mathrm{Tc}$-annexin $\mathrm{A} 5$ have been used: Imino-annexin A5 and BTAP-annexin A5.

We hypothesise that both compounds can be used for imaging of the head-neck region, the thorax and extremities. Until now, published pharmacokinetic and imaging data for Imino-annexin A5 and BTAP-annexin A5 are incomplete, because only their biodistribution has been studied ${ }^{87.105}$. Biodistribution profiles alone are, however, not sufficient to determine target organs for optimal ${ }^{99 \mathrm{~m}} \mathrm{Tc}$-annexin A5 imaging. Moreover, it is useful to compare the initially used Imino-annexin A5 with the second, improved preparation of ${ }^{99 m} \mathrm{Tc}$-annexin A5, BTAP-annexin A5. The purpose of the present study therefore was to characterise and compare the pharmacokinetics and imaging properties of Imino-annexin A5 and BTAP-annexin A5. The study was performed in a group of parients with myocatdial disease. A patient with acute myocardial infarction and patients with different malignancies were studied in order to evaluate the imaging properties of the agents. 


\subsection{Materials and Methods}

\section{Patients}

Imino-annexin A5 was administered to 7 patients (mean age 56 years; tange 47.66 years) and one healthy volunteer ( 35 years). BTAP-annexin A5 was administered to 12 patients (mean age 58 years; range $35-85$ years). Each radiopharmacentical was administered within 4 h of its preparation. All 19 parients had myocardial disease (12 patients had acute myocardial infarction, 4 parients suffered from theart failure, and another three patients had other myocardial diseases (angina pectoris, an intracardial tumor and myocardial contusion, respectively). For imaging purposes four patients suffering from acute myocardial infarction, leg sarcoma, Non-Hodgkin lymphoma and breast cancer were studied. All patients had a normal kidney and liver function. The study was approved by the Medical Ethics Committee of the University Hospital Maastricht.

\section{Radiolabeling}

Both radiolabeled compounds are experimental. An application as IND has been fled for BTAP-annexin A5.

Imino-annexin A5 was prepared by incubating approximately $1000 \mathrm{MBq}$ 99: $\mathrm{T}_{\mathrm{C}}$ pertechnetate with $1 \mathrm{mg}$ freeze-dried, (n-1-imino-mercaptobutyl)-annexin A5 (Mallinckrodt, Petten, The Netherlands) in the presence of $10.8 \mu \mathrm{g} \mathrm{Sn}^{2+}$ as reducing agent for 2 b at ambient temperature. Radiochemical purity was assessed by column chromatography using a Sephadex PD-10 column (Pharmacia, Uppsala, Sweden) and $0.9 \% \mathrm{NaCl}$ (B. Braun, Uden, The Netherlands) containing $1 \%$ bovine albumin (Sigma, St. Louis, MO) as eluent ${ }^{87}$. Imino-annexin A5 was eluted, whereas free pertechnetate and $\mathrm{TcO}$ remained on the column.

BTAP annexin A5 (Apomate@, Theseus Imaging Corporation, Cambridge, MA, USA) was prepared by synthesis of a phentioate ester ligand, followed by coupling to $1 \mathrm{mg}$ annexin $A 5$ and subsequent purification of the obtained BTAP. annexin $A 5$ as previously described ${ }^{105}$. The product was filter sterilised $(0.24 \mathrm{~m}$ pores) after preparation. Purification yield of the Annexin A5-protein was about $80 \%(\approx 800 \mu \mathrm{g})$. The integrity of the filter was checked by a bubble point test. The $\mathrm{pH}$ was measured (limit $\mathrm{pH}$ 6-8) and radiochemical purity was assessed by TLC (ITLC, Gelman Sciences, Ann Arbor, MI) using 12\% trichloroacetic acid (Merck, Darmstadt, Germany) in water as eluent. BTAP-annexin A5 remains at the position of application ( $\mathrm{Rf}=0$ ), whereas most technetium-containing impurities ate at the solvent front $(\mathbb{R f}=1)$. Minor impurities (e.g. colloidal ${ }^{99} \mathrm{~m}$ Tc) were found in the middle section of the TLC glass fiber sheets ( Rf $\approx 0.5$ ). 
Administration of the radiopbarmaceuticals

Imino-annexin A5 (about $2.5 \mathrm{ml}$ ) and BTAP-annexin A5 (about $7 \mathrm{ml}$ ) were given by intravenous bolus injection. Patients received $555 \pm 89$ (mean $\pm \mathrm{SD}$ ) $\mathrm{MBq}$ Iminoannexin $\mathrm{A} 5(1 \mathrm{mg}$ ) or $575 \pm 114 \mathrm{MBq}$ B TAP-annexin $\mathrm{A} 5$ (about $800 \mu \mathrm{g}$ ).

\section{Plasma, urine, and feces sampling}

Blood samples were obtained before administration of the tadiopharmaceutical ${ }_{v}$ and at $5,10,15,20,40,60,90,120,150,180,210$, and 240 min afterwards. From eight patients (BTAP annexin A5 group) one to six additional blood samples were obtained to monitor the clearance of BTAP-annexin A5 over $24 \mathrm{~h}$. The radioactivity in the plasma was measured using a universal gamma counter (1282 Compugamma CS, LKB-Wallack, Turku, Finland) and corrected for background radiation and decay. Urine excreted over $20 \mathrm{~h}$ was collected for BTAP-annexin A5 in two fractions $(0-4 \mathrm{~h}$ and 4-20 h; five patients). Patients were asked to empty their bladders before BTAP. annexin A5 was injected. Feces were collected for BTAP-annexin A5 over the first $20 \mathrm{~h}$.

Plasma annexin A5 concentrations were measured using a validated ELISA (Hyphen Biomed, Andresy, France) according to the manufacturer's protocol. Also, levels of endogenous annexin A5 were measured in a control group of patients with acute myocardial infarction, to detect whether endogenous annexin A5 levels interfere with the measurement of administered Imino-annexin A5 or BTAP-annexin A5. These levels were compared to measurements of endogenous concentrations of Annexin A5 in healthy volunteers.

Determination of the in vivo stability of BTAP-annexin A5

The in wivo stability of BTAP annexin A5 was assessed between 1 and $20 \mathrm{~h}$ after administration of the radiopharmaceutical. One to 2 millilitres of patient plasma (pre-treated with $1 \%$ Triton X-100; Sigma, St. Louis, MO, USA) was added to a Sephadex PD-10 column (Pharmacia, Uppsala, Sweden), pre-eluted with 15 ml eluent (saline containing 1\% bovine albumin; Sigma, St. Louis, MO, USA). The sample was eluted with $7 \mathrm{ml}$ of eluent. The percentage of intact radiolabel was determined by measuring BTAP-annexin A5 in the eluent and unbound pertechnetate retained on the Sephadex PD-10 column.

\section{Biodistribution and pharmacokinetics}

Six (Imino-annexin A5) and five (BTAP-annexin A5) patients underwent imaging with a dual-head gamma camera (Multispect2, Siemens Gammasonics, Hoffman Estate, lL). The gamma camera was equipped with high-resolution collimators, and a $15 \%$ energy window around the photo peak of ${ }^{99} \mathrm{Tc}$ was used. On injection of the radiopharmaceurical, a dynamic study of the thorax region was started, lasting 30- 
60 min. Subsequently, whole-body scans were performed with a scan speed of 10 $\mathrm{cm} / \mathrm{min}$, two to four scans for the Imino-annexin A5 group and three scans for the BTAP-annexin A5 group. 'The whole-body acquisitions were typically performed at 2,4 , and $20 \mathrm{~h}$ after radiopharmaceutical administration.

The pharmacokinetic parameters were derived using the KINFIT module of the MW/ PHARM computer program package (Version 3.30, MediWare, Groningen, The Netherlands) ${ }^{134}$. The data, consisting of the plasma concentration of ${ }^{99 \mathrm{~mm}} \mathrm{~T}_{\mathrm{C}}$ radioactivity or annexin A5 versus time, were analysed by non-linear regression analysis using a least-squares weighted simplex algorithm, with data weighted with the reciprocal of the observed value. The following parameters were obtained: $\tau_{s, \alpha^{2}}$,

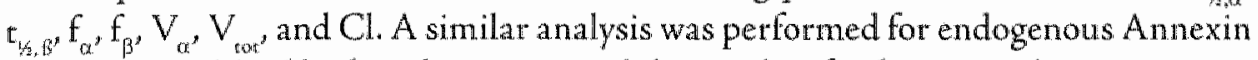
A 5 in plasma. Total body radioactivity and the uptake of radioactivity by organs were calculared as previously described ${ }^{87.105}$.

\section{SPECT and/or planar imaging of patients}

\section{Patient with acute myocardial infarction}

The diagnosis of acute myocardial infarction was made by electrocardiographic criteria and confirmed by biochemical detection of cardiac enzyme release. Iminoannexin $A 5(638 \mathrm{MBq})$ was administered intravenously $2 \mathrm{~h}$ after reperfusion, which was achieved by performing PTCA, resulting in thrombolysis in myocardial infarction (TIMI) III How. Images were obtained $22 \mathrm{~h}$ after injection. A $64 \times 64$ matrix was used and 60 angle views, counting $45 \mathrm{~s}$ at each angle. Studies were reconstructed using a filtered back-projection method, using a Butterworth filter with a cut-off frequency of 0.55 and an order of 5. All SPECT studies were converted to transverse slices to compare the reperfusion defect with the uptake of Imino-annexin A5. After 7 weeks, sestamibi imaging was performed to evaluate the reperfusion defect ${ }^{56}$.

\section{Sarcoma of the leg}

A 58 year old woman was admitted for resection of a large tumor in the upper right thigh (approx. $9 \times 9 \times 18 \mathrm{~cm}$ ). Ten months earlier surgical biopsy diagnosed the tumor as a soft tissue sarcoma; of the epithelioid type. Further evaluation revealed lung metastases. Chemotherapy (doxorubicine 3 times $75 \mathrm{mg} / \mathrm{m}^{2} ; 6$ months before operation), regional perfusion with melfalan and TNF- $\alpha$ ( 3 months before operation) and radiotherapy ( 3 times $6.0 \mathrm{~Gy} ; 1$ to $2 \frac{1}{2}$ weeks before operation) were without effect on tumor size and lung metastases (as evaluated by MRI). Because of local tumor progression with ulceration, a hip-exarticulation of the right leg was planned as a palliative procedure. One day prior to the operation $400 \mathrm{MBq}$ of BTA.P-annexin A5 was administered i.v. followed by static images after 4 (including SPECT) and 16 hours. 
Breast carcinoma

A 37 year old female was diagnosed with mastitis carcinomatosa. Furthermore, left axillary palpable lymph nodes were found. Further metastatic disease was not detectable. Prior to chemotherapy, $603 \mathrm{MBq}$ BTAP-annexin A5 was injecred intravenously to determine the apoptotic status of the disease. SPECT scintigraphy was performed $5 \mathrm{~h}$ and $15 \mathrm{~h}$ after administration of the radiopharmaceutical.

Non-Hodgkin Lymphowa

A 63 year old male was diagnosed with a grade 1 small cell follicular Non-Hodgkin Lymphoma. A CT scan of the thorax revealed a large mass in the neck region. Prior to chemotherapy, $391 \mathrm{MBq}$ of BTAP-annexin A5 was injected intravenously in order to determine the apoptotic status of the disease in this patient. SPECT scintigraphy was performed 4 and $8 \mathrm{~h}$ after administration of BTAP-annexin A5.

Data were statistically evaluated using Student's t-test. A probability value $<0.05$ was considered significant. Results are generally presented as means $\pm \mathrm{SD}$. 


\subsection{Results}

Radiolabeling of annexin A5 yielded Imino-annexin A5 and BTAP-annexin A5 with a radiochemical purity of $79 \pm 3 \%$ and $96 \pm 2 \%$, respectively. The radiolabeling procedures did not affect the phosphatidylserine binding ability of Annexin A5 as evaluared by ellipsometry ${ }^{26}$.

Both radiopharmaceuticals were stable for up to $4 \mathrm{~h}$ after their preparation. No side effects were observed after the administration of the radiopharmaceuticals to the patients.

After evaluation of the data for patients receiving Imino-annexin $\mathrm{A} 5$, it was decided to collect additional information (urine and feces samples, stability in plasma) for BTAP-annexin A5.

Figure 3.1 (top) shows the time courses of the radioactivity in plasma for Iminoannexin A5 and BTAP-annexin A5. The annexin A5 antigen concentrations versus time curves are shown in the bottom panel. The kinetics of both radiolabeled annexin $\mathrm{A} 5$ and annexin A5 antigen in plasma could satisfactorily be described by a two-compartment model. Curve fitting of the pharmacokinetic data with a threecompartment model did not improve the fit as can be judged from the $\mathrm{r}^{2}$ for both the 2-compartment and 3-compartment model in Table 3.1. The latter table gives the kinetic parameters for Imino-annexin A5 and BTAP-annexin A5 in plasma, assuming a two-compartment model. The calculated clearance of BTAP-annexin A5 $(2.8 \pm 1.2 \mathrm{l} / \mathrm{h})$ was significantly higher than that of Imino-annexin $\mathrm{A} 5(1.2 \pm 0.4 \mathrm{l} / \mathrm{h}$; $\mathrm{p}<0.01$ ). The fast $\alpha$-component of Imino-annexin A5 radioactivity in plasma ( $52 \pm$ $13 \%$ ) was significantly smaller than the corresponding component of BTAP-annexin A5 $(81 \pm 13 \%) ; \mathrm{p}<0.001$. 

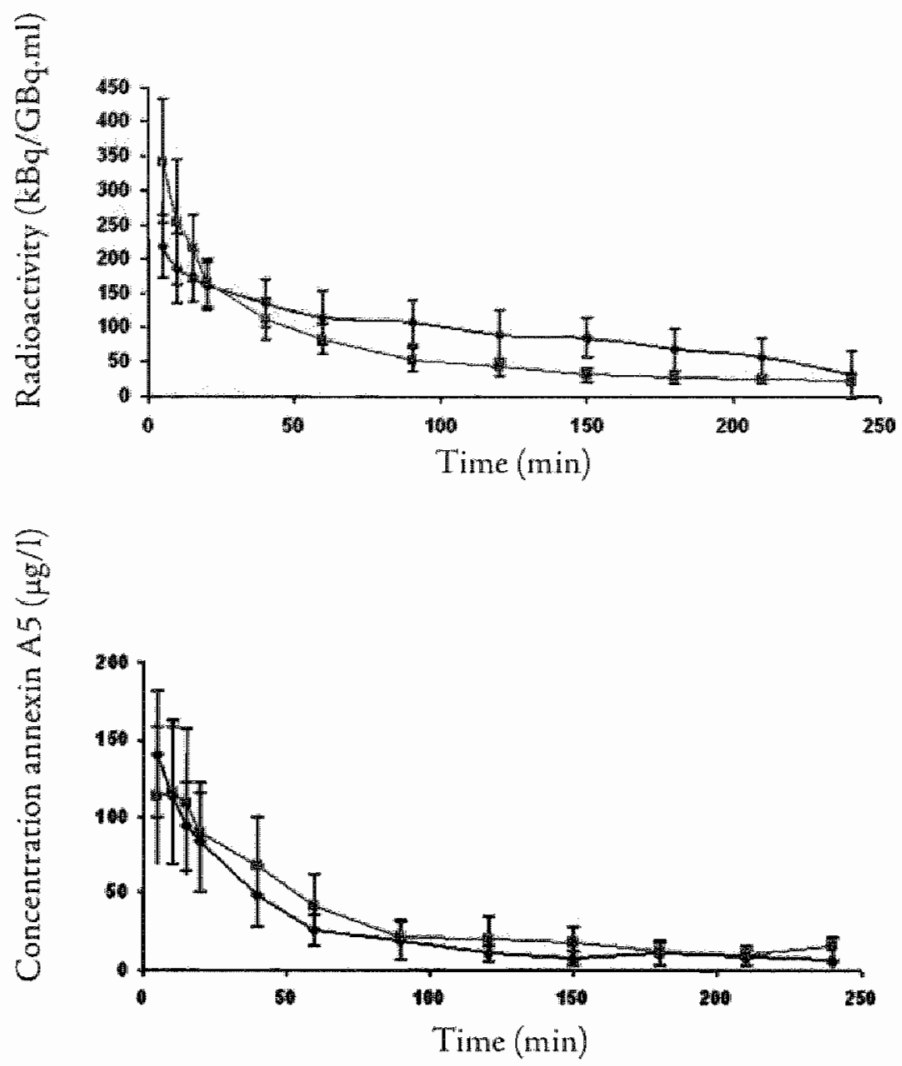

Figure 3.1. Concentration-time curves of T-annexin A5 ( ) and BTAP-annexin A5 ( 99 Tc radioactivity in plasma (upper panel) and annexin A5 concentration in plasma (lower panel). For Imino-annexin A5, data for 8 individuals are presented, whereas for BTAP. annexin $A 5$ values for 10 (radioactivity) and 12 (plasma concentration annexin A5) patients are given. Radioactivity data were nomalised with the administered activity by dividing the obtained plasma radioactivity levels by the administered dose in $\mathrm{GBq}$. All values are depicted

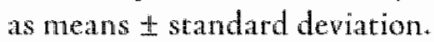

Plasma levels of annexin A5 antigen ranged from 4 to $104 \mu \mathrm{g} / \mathrm{l}(\mathrm{1}$ h after administration) in patients injected with BTAP-annexin A5. Because of the wide range of dara, median and ranges are given for the half-life and the volume of distribution of the radiopharmaceuticals in the elimination phase. In the control group of patients who did not receive the radiopharmaceutical, plasma levels of annexin A5 ranged from 3-68 $\mu \mathrm{g} / \mathrm{l}$. In contrast, annexin A5 levels were much lower in healthy volunteers, $0-9 \mu \mathrm{g} / 1$ (W.L. van Heerde, private communication, 2001) (Table 3.2, see next page). 


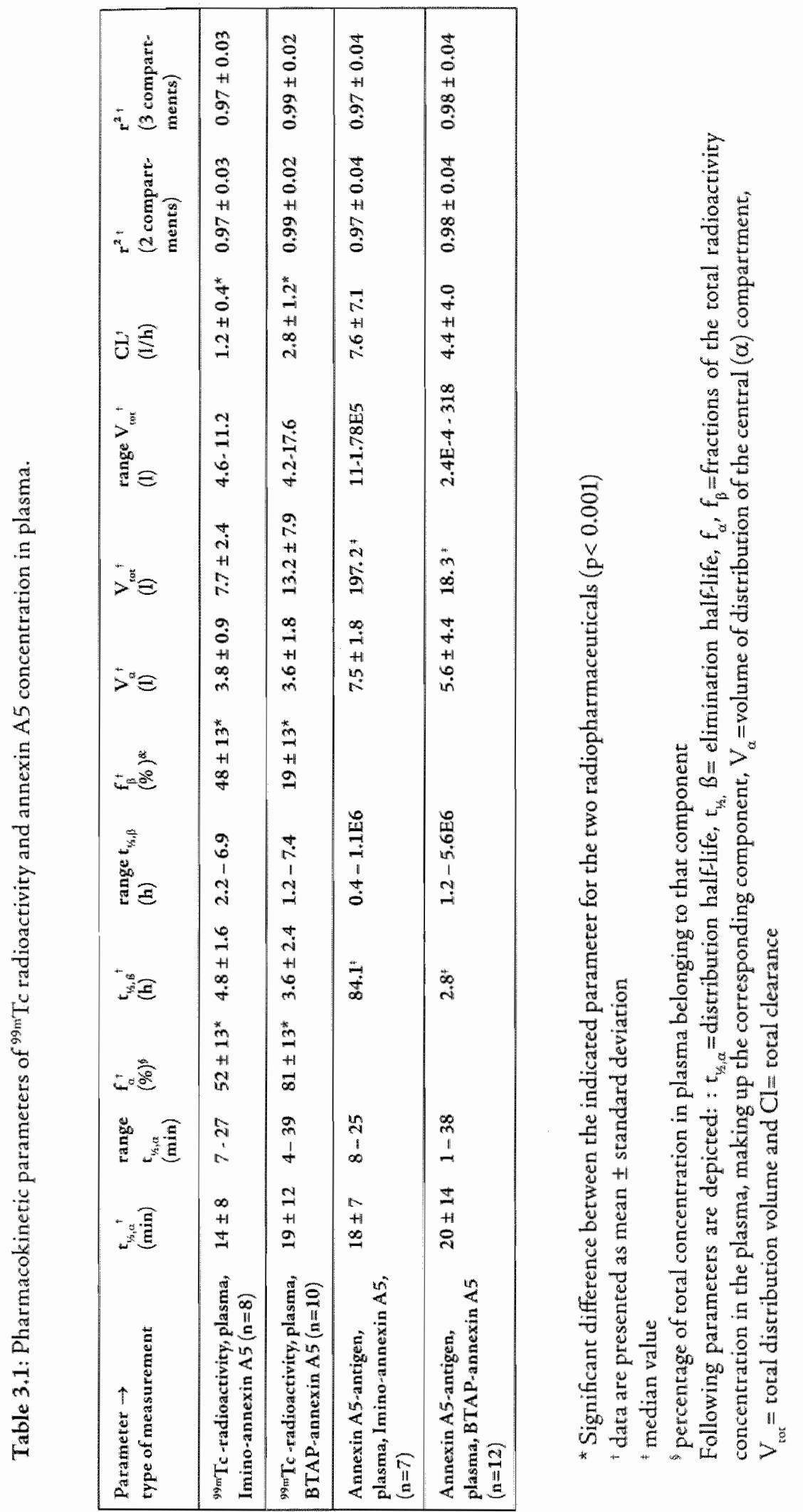


More than $75 \%$ of BTAP-annexin A5 present in plasma remained chemically stable for at least 20 h after administration of the radiopharmaceutical (Table 3.3).

Table 3.2: Annexin A5 levels in patients with myocardial disease and in healthy volunteers

\begin{tabular}{lll}
\hline Type of individual & $\begin{array}{l}\text { Range (mean) of } \\
\text { annexin A5-concentration } \\
(\mu \mathrm{g} / \mathrm{L})\end{array}$ & $\begin{array}{l}\text { Number of } \\
\text { individuals }\end{array}$ \\
\hline $\begin{array}{l}\text { Myocardial disease, Imino-annexin A5 or } \\
\text { BTAP-annexin A5, measured 1-24 h after } \\
\text { administration. }\end{array}$ & $4-104(\mathbf{1 0})$ & $\mathrm{N}=\mathbf{1 7}$ \\
$\begin{array}{l}\text { Myocardial infarction, no radiolabeled } \\
\text { annexin A5, measured until 8 h after } \\
\text { diagnosis }\end{array}$ & $3-68(10)$ & $\mathrm{N}=29$ \\
\begin{tabular}{l} 
Healthy volunteers \\
\hline
\end{tabular}
\end{tabular}

"W.L. van Heerde, University of Maastricht, private communication, 2001.

Representative whole-body scintigrams, obtained after administration of Iminoannexin A5 and BTAP-annexin A5, are given in Figure 3.2 to illustrate the biodistribution of the two tadiopharmaceuticals. There was a high uptake of radioactivity in the kidneys and the liver. The faster excretion of BTAP-annexin A5 radioactivity is shown by the earlier presence of radioactivity in the gut in the images to the right in Figure 3.2. Cardiac uptake of radiolabeled annexin A5 was seen in patients with acute myocardial infarction (Figure 3.4, see page 101) and in a patient with an intracardial tumor ${ }^{67}$. Cardiac tracer uptake was assessed visually, because it was not possible to quantify the cardiac accumulation of tadiolabeled annexin A5 due to its low radiation intensity compared to the radiation in adjacent organs such as liver and kidneys.

Figure 3.3 depicts the clearance of Imino-annexin A5 (upper panel, $n=6$ ) and I-BTAP. annexin $A 5$ (lower panel, $n=5$ ) from the body. BTAP-annexin $A 5$ was excreted faster than Imino-annexin A5: the biological half-life of BTAP-annexin A5 was $17 \pm 8 \mathrm{~h}$ and that of Imino-annexin $A 5$ was $62 \pm 13 \mathrm{~h}(\mathrm{p}<0.001)$. Data from five patients showed that $26.0 \pm 9.3 \%$ of injected BTAP-annexin A5 was excreted in urine up to $4 \mathrm{~h}$ and $57 \pm 11 \%$ up to $20 \mathrm{~h}$. The amount of radioactivity excreted in feces in $20 \mathrm{~h}$ was estimated to be about $25 \%$ for both radiopharmaceuticals. 
Table 3.3: Percentage intact radiolabel for BTAP-annexin A5 in plasma of patients

\begin{tabular}{lll}
\hline Time post injection & $\begin{array}{l}\text { Intact } \\
\text { radiolabel }(\%)\end{array}$ & Number of patients \\
\hline $1 \mathrm{~h}$ & $88.3 \pm 3.7$ & $\mathrm{~N}=9$ \\
$2 \mathrm{~h}$ & $82.5 \pm 5.5$ & $\mathrm{~N}=6$ \\
$4 \mathrm{~h}$ & $78.3 \pm 2.8$ & $\mathrm{~N}=4$ \\
$20 \mathrm{~h}$ & $90.1 \pm 1.5$ & $\mathrm{~N}=4$ \\
\hline
\end{tabular}

"Even in the case of the same group size, part of the patients may be different

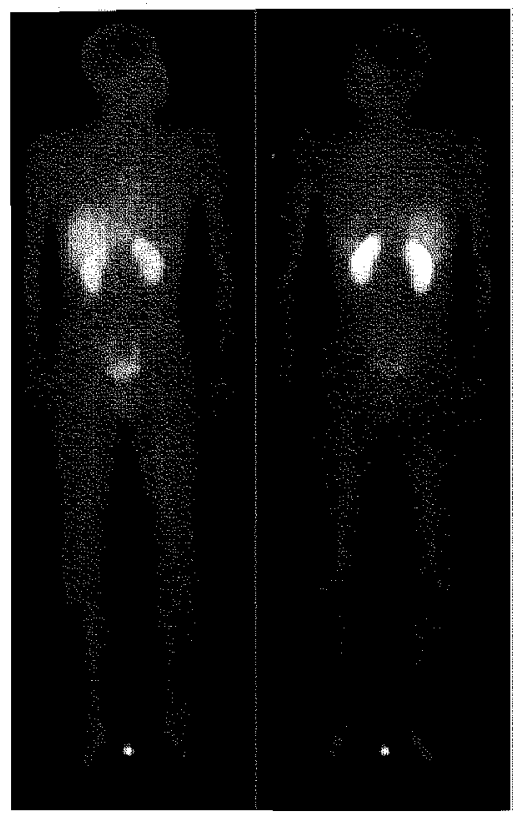

A

P

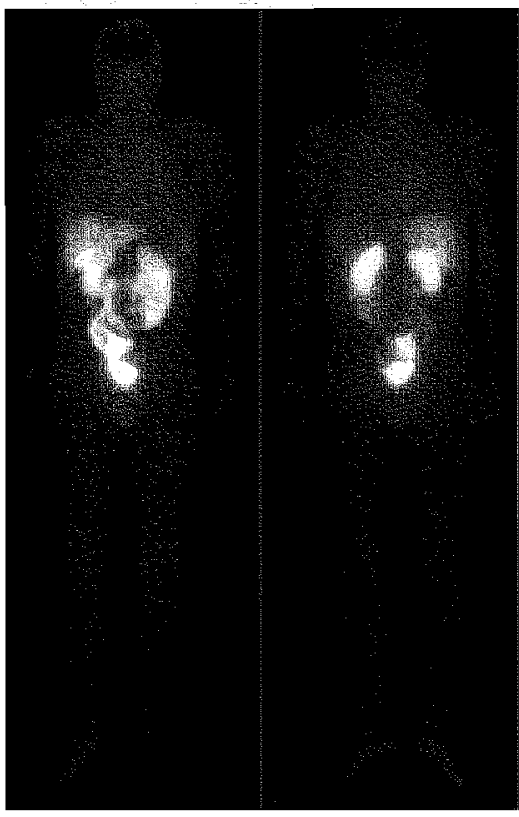

A

$\mathbf{P}$

Figure 3.2: Views of whole-body scan of a male volunteer ( 35 years, left) and a male patient ( 53 years, right), taken between 4 and 5 hours after administration of $440 \mathrm{MBq}$ Imino-annexin A5 and $566 \mathrm{MBq}$ BTAP-annexin A5, respectively. A: anterior view" P: posterior view. 

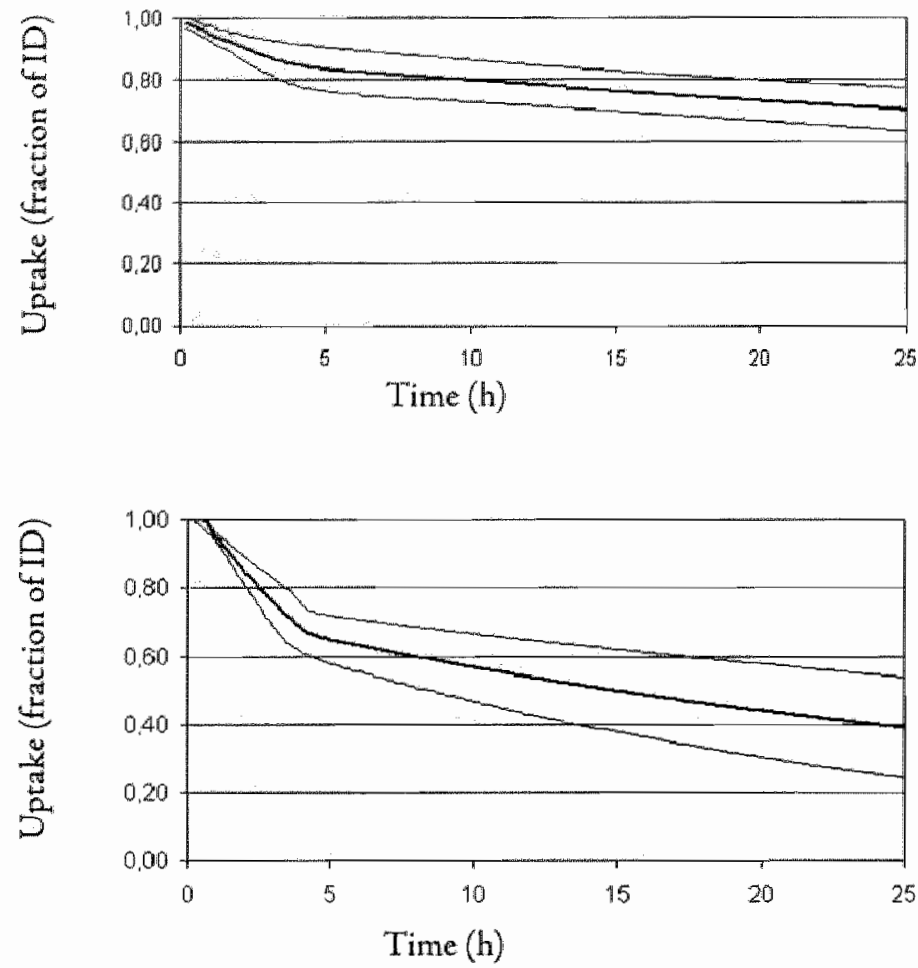

Figure 3.3: Decay corrected ${ }^{99 \mathrm{~m}^{\top}} \mathrm{T} \mathrm{c}$-uptake in the total body after injection of Imino-annexin A5 (upper panel) and BTAP-annexin A5 (lower panel), obtained with the gamma-camera. Curves with mearn and [mean $\pm 1 \mathrm{SD}]$ are shown. $\mathrm{ID}=$ injected dose.

In vivo apoptosis detection

\section{Acute myocardial infarction}

Figure 3.4 shows an example of Imino-annexin A5 uptake in the anteroseptal area of the patient with an acute anterior wall myocardial infarction at $22 \mathrm{~h}$ after injection ${ }^{66}$.

Sarcoma of the leg

Planar images at 16 hours after injection of BTAP-annexin A5 clearly show increased activity in the right upper leg of the patient with sarcoma, indicating PS expression in this region. The latter is best seen in the anterior image (Figure 3.5). The central region without activity corresponds to an area of necrosis on a CT scan (Posterior image, Figure 3.5).

Breast carcinoma

Figure 3.6 (see page 102) shows several SPECT images, obtained $5 \mathrm{~h}$ after injection, 
of BTAP-annexin A5 from the patient with mastitis carcinomatosa of the breast. All generated SPECT views show clearly uptake of BTAP-annexin A5 in the anterior part of the left breast, indicative for PS-expression in this tumor (indicated by the arrows).

Non-Hodgkin Lymphoma.

As an example a localisation of a Non-Hodgkin Lymphoma in the neck is shown in Figure 3.7. The picture indicates that BTAP-annexin A5 is taken up by the lymphoma, 8 h after intravenous injection of the radiopharmaceutical. 


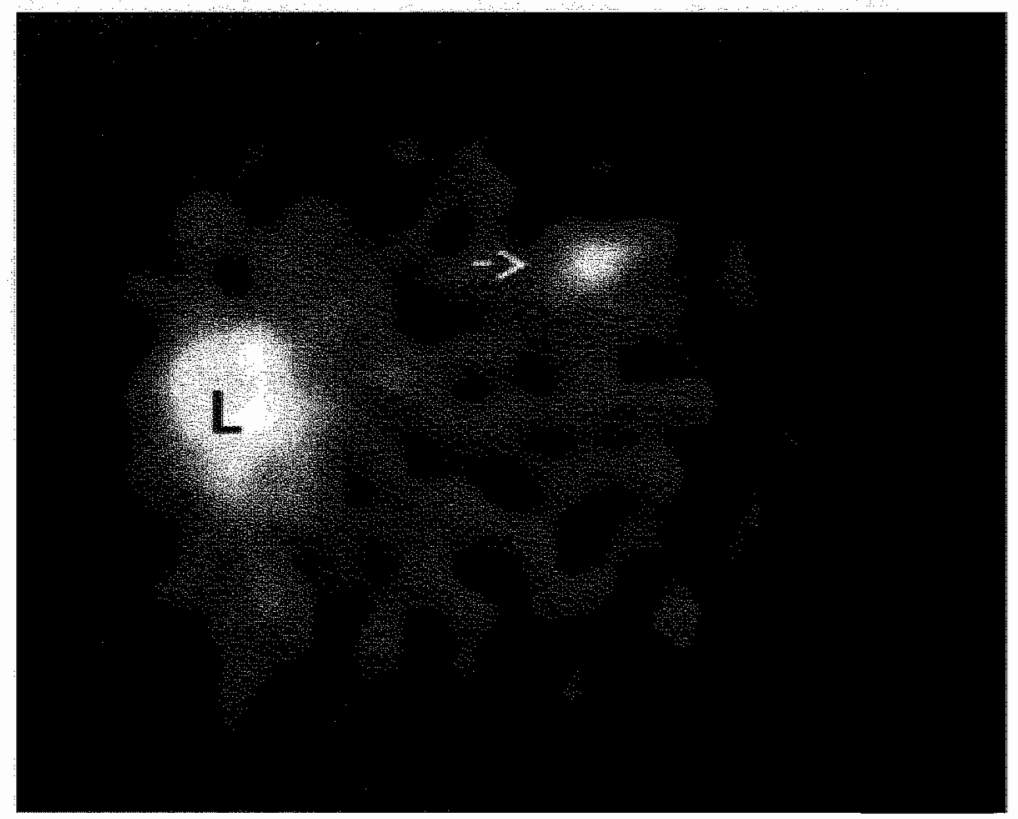

Figure 3.4: Transverse tomographic image obtained with Imino-annexin A5 in a patient suffering from acute anteroseptal myocardial infarction. The arrow indicates increased Iannexin $A 5$ uptake in the anteroseptal tegion $22 \mathrm{~h}$ after reperfusion
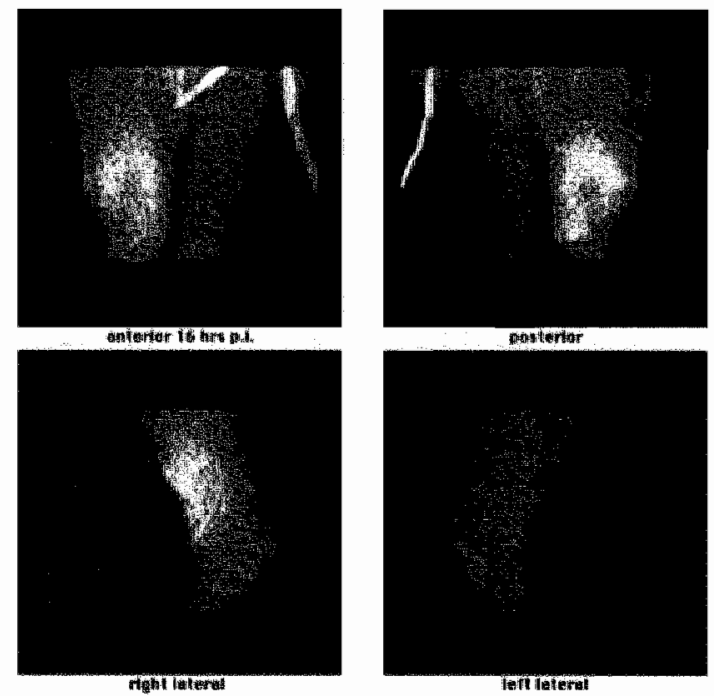

Figure 3.5: Planar images of a patient with leg sarcoma, 16 h after the injection of $B$-annexin A5. There is increased uptake of BTAP-annexin A5 in a majot part of the upper right leg, with a central zone of decreased activity. Almost no uptake was seen in the left leg. 


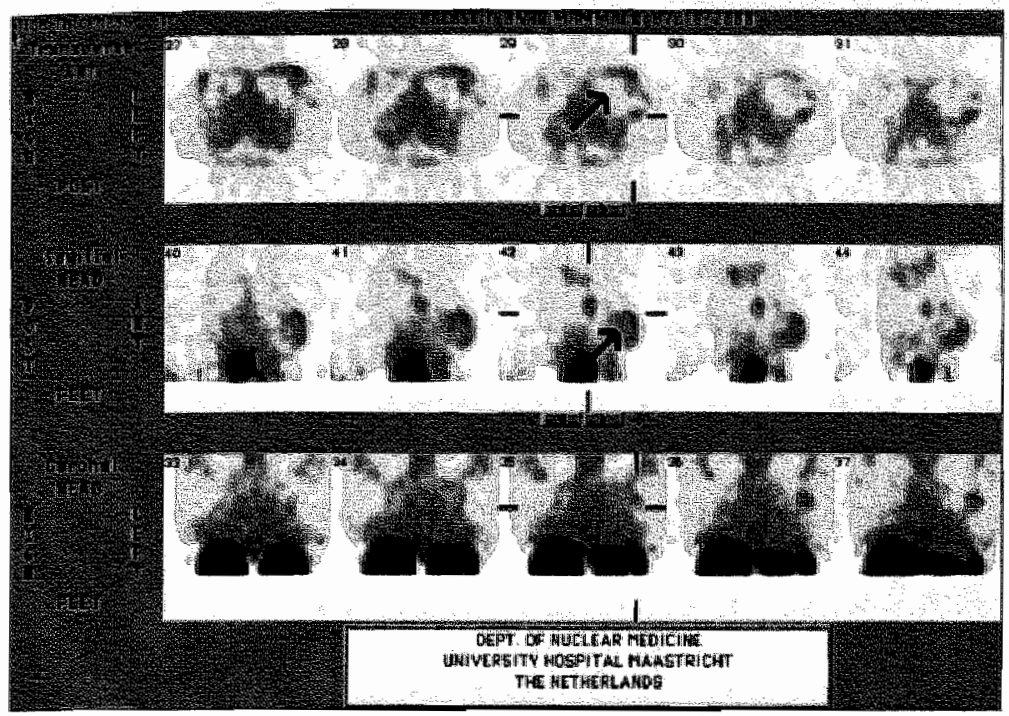

Figure 3.6: SPECT (5 h post injection of BTAP-annexin A5) images of a patient with mastiris carcinomatosa. The increased uptake, of BTAP-annexin A5, indicated by arrows, can be seen in the anterior part of the left mamma.

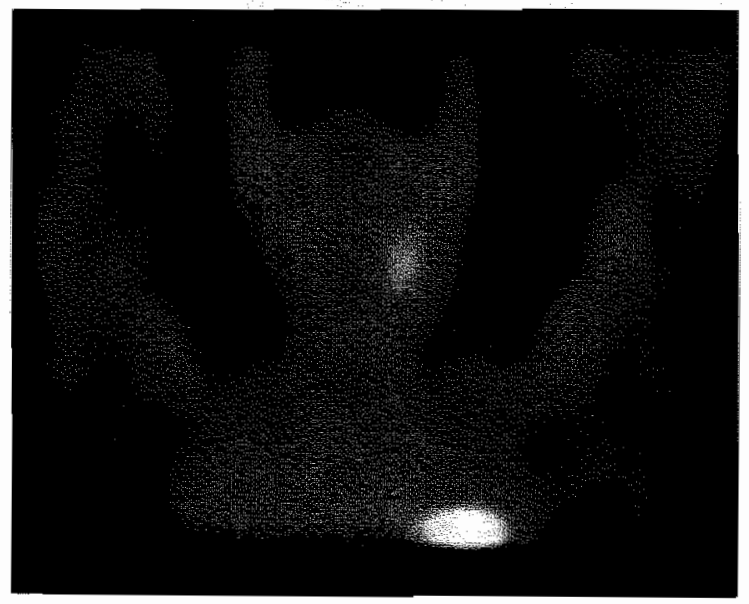

Figure 3.7: Coronal SPECT image of a patient with Non-Hodgkin Lymphoma. Increased BTAP-annexin A5 accumulation is seen in the neck region, corresponding to a latge mass on a C I-imaging. 


\subsection{Discussion}

This paper describes characteristics of two different preparations of radiolabeled Annexin A5. The procedure yielding Imino-annexin A5 is much easier to perform than the procedure giving BTAP-annexin A5. Both radiopharmaceuticals showed different radiochemical and pharmacokinetic characteristics. First, Imino-annexin A5 was less pure than BTAP-annexin A5. The rather low radiochemical purity of Imino-annexin $A 5$, which decreased from $83 \%$ to $76 \%$ over 6 months, is probably due to the decrease in the amount of $\mathrm{Sn}^{2+}$ in the kit with time.

The radiochemical purity of BTAP-annexin A5 remained stable for the prescribed shelf-life of 12 weeks. The low radiochemical purity of Imino-annexin A5 might possibly affect the radiation dose administered, pharmacokinetics and imaging results. Assuming that the free ${ }^{99 m} \mathrm{Tc}$ in Imino-annexin $\mathrm{A} 5$ is present in the form of pertechnetate, the effect on the patient effective dose is small ( $<10 \%$ increase $)^{87}$. Also, the effect of radiochemical impurities on the pharmacokinetics of Imino-annexin A5 is small. After correction of the plasma data for free ${ }^{99 m} \mathrm{Tc}$ pertechnetate, by subtraction of the weighted pharmacokinetic curve for pertechnetate ${ }^{135}$, the difference in amplitude of the $\alpha$-and $\beta$-components between the two radiopharmaceuticals was still present. "This suggests that the radiochemical instability of Imino-annexin A5 did not affect its biodistribution and imaging properties.

Owing to the radiochemical problems with Imino-annexin A5, the stability of BTAPannexin A5 was investigated more extensively. It was stable in vivo, for at least $20 \mathrm{~h}$ after injection (Table 3.3).

The clearance of the two radiopharmaceuticals from plasma was also different. Although for the kinetics in both cases similar half-lives were found for the corresponding components, the relative magnitude of the components differed significantly berween Imino-annexin A5 and BTAP-annexin A5 $(p<0.001)$. The large amplitude of the $\alpha$-component for BTAP-annexin A5 corresponds to the large initial decline in Figure 3.1 (top). The greater amplitude of the $\beta$-component for Imino-annexin A5 is reflected in Figure 3.2, where the large blood vessels are still visible at $4-5 \mathrm{~h}$ after injection of Imino-annexin A5, but not after injection of BTAP-annexin A5. Kemerink et al. reported a somewhat larger $t_{\mathrm{k}, \alpha}(26 \pm 5 \mathrm{~min})$ and $t_{i, \beta}(6.9 \pm 1.4 \mathrm{~h}$ ) for BTAP-annexin A5 in the blood of five patients (See Chapter 4) ${ }^{105}$. This difference is mainly due to the use of different measurement times in the two studies. While Kemerink et al. measured radioactivity in the blood up to $20 \mathrm{~h}$, we measured samples up to $4 \mathrm{~h}$ after administration. This finding suggests that the pharmacokinetic model must be more complicated than a simple two-compartment model. Nevertheless, a two-component model gave a satisfactory fit for data for all time points, whereas a three-component model did not provide a better fit, as judged from $r^{2}$ (Table 3.1). The pharmacokinetics of ${ }^{123} \mathrm{I}$-annexin $\mathrm{A} 5$ in pigs were also described best by a two-compartment model $\left(\mathrm{c}_{\mathrm{s}, \mathrm{\alpha}}=6.4 \mathrm{~min}, \mathrm{t}_{\mathrm{s}, \mathrm{k}, \mathrm{R}}=71 \mathrm{~min}\right)^{93}$. In our 
study, both radiopharmaceuticals showed similar pharmacokinetics for annexin A5antigen (Table 3.1). This is probably because most of the administered annexin A5 $(>99 \%)$ was not labelled with ${ }^{99} \mathrm{~m} \mathrm{~T}$, and thus the unlabeled annexin A5 should thave the same biochemical properties for both preparations. Although the labeling with ${ }^{99 m}$ Tc clearly affected the kinetics of the antigen, this could not be observed for the whole pool of annexin A5 because $99 \%$ of annexin A5 was nor labeled.

The kinetics of annexin A5-antigen were difficult to describe $>1$ h after radiopharmaceutical administration. Because this could be caused by fuctuarions in endogenous annexin A5 levels, we decided to compare plasma annexin A5 concentrations in patients receiving $\mathrm{BTAP}$-annexin $\mathrm{A} 5$ and in patients with a myocardial infarction not receiving the radiopharmaceutical (Table 3.2). In both groups annexin $\mathrm{A} 5$ levels showed a broad variation. Corresponding values were much lower in a group of healthy volunteers in whom annexin $A 5$ antigen levels had been determined before (Table 3.2). Thus measurement of the concentration of radiolabeled annexin $\mathrm{A} 5$ antigen seems to be hampered by fluctuations in endogenous annexin A5 levels. This Auctuation in the concentration of annexin A5 in patients with myocardial infarction has been described befor ${ }^{56}$. At present, it is still not clear what causes these fuctuations.

The biological half-life in the body was $62 \pm 13 \mathrm{~h}$ for Imino-annexin A5 and $16 \pm 7$ $\mathrm{h}$ for BTAP-annexin A5, which is not oprimal for imaging purposes. The excretory pathway for both radiopharmaceuticals was identical. Extrapolation of data obtained in the first 20 h showed that about $25 \%$ of the radiopharmaceutical is excreted in feces and the temainder in urine. Recently, the biodistribution of annexin A5 derived mutants containing endogenous chelation sites labelled with ${ }^{99} \mathrm{~m} T \mathrm{C}$ was described in mice. Compared with Imino-annexin A5 and BTAP-annexin A5, these mutants show considerably lower uptake in the liver and a similar to lower uptake in the kidney measured in the same time frame after administration. No pharmacokinetic data were shown".

The difference in pharmacokinetics between Imino-annexin A5 and BTAP-annexin A5 must be due to the different ligands used to attach ${ }^{99 \mathrm{~m}} \mathrm{Tc}$ to the annexin A5 molecule. In Imino-annexin A5 an n-1-imino-4-mercaptobutyl group is used, which is chemically unrelated to the 4,5-bis(thioacetamido)pentanoyl group used for incorporating ${ }^{99_{m}} \mathrm{Tc}$ in BTAP-annexin A5. Changes in biodistribution and pharmacokinetics caused by differences in conjugation methods have been described before for radioimmunoconjugates ${ }^{136}$.

Furthermore, the high uptake of the two annexin A5 tadiopharmaceuticals in the liver and kidneys, and in the case of BTAP-annexin A5 the relatively fast appearance of radioactivity in the gut, hampers imaging of the abdomen. "The study of the thorax and the head/neck region appears feasible ${ }^{105}$. Here, BTAP-annexin A5 has the advantage of its faster clearance, whereas Imino-annexin $A 5$ would be more favorable for imaging of the abdomen. 
We were able to show that ${ }^{99 m} T c$-annexin $A 5$ is suitable for imaging several targets: acute myocardial infarction and malignant tumors. The imaged tumors were confirmed with CT imaging and histology, indicating that the observed PSexpression is related ro the tumor cell death programme. As can be judged from the additional information present in this applied scintigraphy, the optimal imaging time generally depends on the target to be imaged. A SPECT study revealed the best time for imaging of myocardial infarction to be between 15 and $20 \mathrm{~h}$ after administration of the radiopharmaceutical ${ }^{66}$. The imaging studies of tumors in the extremities and in the female breast suggest that this kind of scintigraphy can be performed best 5 $15 \mathrm{~h}$ after administration of BTAP-annexin A5, dependent on the tumor type. For imaging of cell death, an agent with no apparently non-specific uptake in organs like liver, kidney and gut would be favorable. This goal seems hard to achieve but remains an essential target for further research. 


\subsection{Conclusion}

This chapter describes the pharmacokinetic and imaging properties of Imino-annexin A5 and BTAP-annexin A5, two radiopharmaceuticals suitable for the detection of apoptosis by in vivo imaging of the heart, extremities, breast and head-neck region. Firstly, it should be stressed that the behavior of the ${ }^{99 m}$ Tc labelled annexin A5 protein in the human body is affected by the labeling method used. Secondly, BTAPannexin $\mathrm{A} 5$ is the most stable radiopharmaceutical with good and reproducible labeling characteristics. Labeling of pertechetate with Imino-annexin A5 however was less reliable and the obtained radiochemical purity lower. BTAP-annexin A5 has the advantage of a somewhat faster clearance from the body. Imaging of apoptosis in the abdomen will be difficult with both radiopharmaceuticals, and especially with BTAP-annexin A5 because of its faster appearance in the gut.

This study was supported in part by grants from the Dutch Heart Foundation (NHS 98.125 and NHS D96.025). 


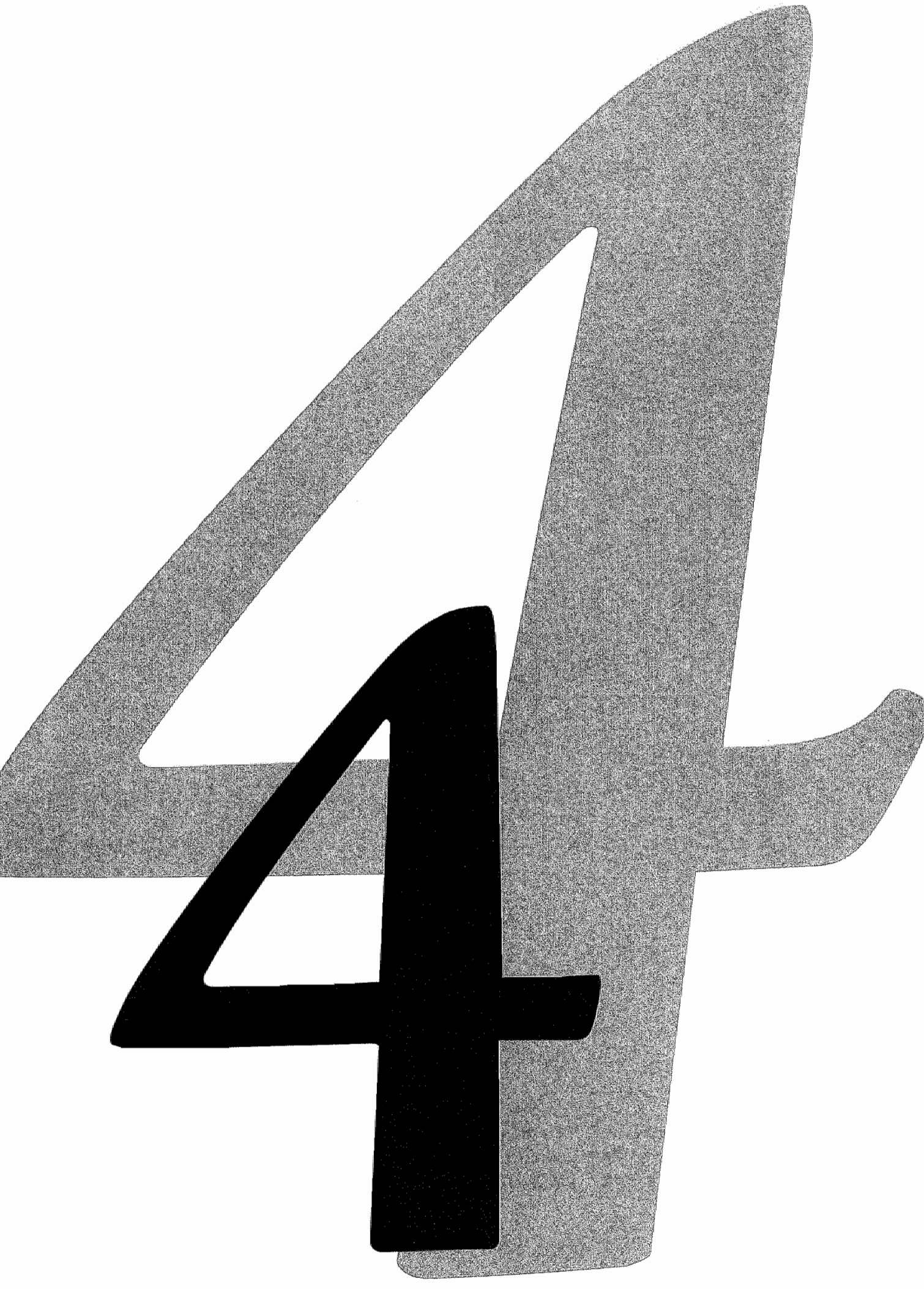





\section{Biodistribution and dosimetry of ${ }^{99_{\mathrm{m}}} \mathrm{Tc}$-BTAP- annexin $A 5$ in humans}

Adapted from: G.J. Kemerink, H.H. Boersma, P.W.L. Thimister, L. Hofstra, I.H. Liem, M-Th. Pakbiers, D.Janssen, C.P.M. Reutelingsperger, G.A.K. Heidendal. Biodistribution and dosinetry of 99 TC-BTAP-annexin $V$ in bumans. Eur J Nud Med 2001:28:1373-8. 


\subsection{Introduction}

Programmed cell death (PCD), is involved in homeostasis as well as in a number of diseases. It plays a key role in embryogenesis, carcinogenesis, viral disease, ischemia and autoimmune disorders, and the beneficial or detrimental effect of several drugs can be attributed to their action on the apoptotic process. Changes in the rate and extent at which apoptosis occurs could therefore be important medical information. For this reason considerable effort is spent in developing and validating methods to image PCD in vivo.

One important potential application of monitoring apoptosis is showing the success or failure of chemo- and radiation therapy in cancer treatment ${ }^{132}$. Several other potential applications exist, however, including the identification of hypoxic-ischemic brain or myocardial injury, screening for neurodegenerative diseases, the study of arherosclerotic plaques, the study of the efficacy of cell protecting pharmaceuticals in ischemia, monitoring allografts and following the effect of anti-tejection drugs ${ }^{132}$.

Annexin A5 is a protein with a high affinity for negatively charged phospholipids ${ }^{37}$. Actions due to its affinity for phospholipids include binding to phosphatidyl serine on the outer leaflet of cell membranes during apoptosis ${ }^{81}$. The externalisation of phosphatidyl serine is an early and persisting process in apoptotic cells ${ }^{59}$. The use of annexin A5 as a marker of cell death using radiolabeled and fluorescent annexin A5 has been developed in vitro and in vivo in animal models ${ }^{65,100}$. Recently, we successfully applied ${ }^{99 m} \mathrm{Tc}$-(n-1-imino-4-mercaptobutyl)- annexin A5 (Iminoannexin A5, Mallinckrodt, Petten, The Netherlands) in imaging of cell death in patients with an acute myocardial infarction ${ }^{66}$. Unfortunately, the production of this compound has been stopped. This forced us to switch to an alternative preparation, 99in'Tc-4,5-bis(thioacetamido) pentanoyl-annexin-V (Apomate, ${ }^{49}$ 'Tc-BTAP-annexin A5, BTAP-annexin A5; Theseus Imaging Corporation, Cambridge, MA), which is available for investigational purposes. Due to the different ligand used for the binding of the radioisotope, BTAP-annexin A5 behaves differently from Imino-annexin A5. Here we report the biodistribution and dosimetry of BTAP-annexin A5; similar information for Imino-annexin A5 has been reported previously ${ }^{87}$. 


\subsection{Materials and Methods}

Subjects

Eight patients participated in this study, including two females. The average age was $53 \pm 15$ years (range 37-85). Two patients had non-Hodgkin lymphoma, two suffered from subacute myocardial infarction, three from decompensatio cordis, and one had Hodgkin's disease. Only patients with normal liver and kidney function were included in this study. The study was approved by the local medical ethics committee and all patients gave informed consent.

\section{Radioactive tracer}

The tracer ${ }^{99 m}$ Tc-4,5-bis(thioacetamido)pentanoyl-annexin A5 (BTAP-annexin A5; Theseus Imaging Corporation, Cambridge, MA) has been approved by the FDA for investigational purposes. For details concerning its preparation and quality control we refer to the protocols provided as part of the kit. Briefly, the radioactive tracer is prepared in a number of steps. The ${ }^{99 m} \mathrm{Tc}-4,5-b i s$ (thioacetamido) pentanoylannexin A5 ester was synthesised by first teducing $4 \mathrm{GBq}^{99 \mathrm{~m}}$ Tc-pertechnetate in the presence of the weak chelating agent stannous gluconate, followed by incubation with the phenthioate reagent at $70 \pm 5^{\circ} \mathrm{C}$ during 15 minutes. Afrer cooling, $1 \mathrm{mg}$ of human recombinant annexin $\mathrm{A} 5$ was added, and the mixture allowed to react at room temperature during 20 minutes. The reaction product was divided over two Sephadex G-25 columns for purification, and after elution using $8 \mathrm{ml}$ of phosphate buffered saline as eluant, forced through a sterile filter with $0.2 \mu \mathrm{m}$ pores. Integrity of the filter was checked by subjecting it to a bubble point test (pressure minimum > 3 bar). Quality control of the tracer was performed by measuring the $\mathrm{pH}$ of the filtrate (limits 6-8), and by assessing radiochemical purity by thin layer chromatography using silica impregnated paper (ITLC, Gelman Sciences, Ann Arbor, MI) and 12 $\%$ trichloroacetic acid (Merck, Darmstadt, Germany) in water as eluant. The tracer BTAP-annexin A5 stays at the origin ( $\mathrm{R}=0)$, most tednetium containing impurities are at the solvent front $(R f=1)$, and collodial technetium is found somewhere halfway ( $R f \approx 0.5$ ). Finally, BTAP-annexin A5, containing about $800 \mu \mathrm{g}$ of annexin $\mathrm{A} 5$ and

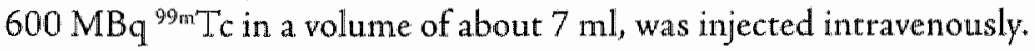

\section{Data acquisition}

Although imaging and data analysis have been described before, the more important aspects will be summarized here for the convenience of the reader. A number of adaptations that were introduced are presented as well. Imaging was performed with a dual head gamma camera, yielding conjugate anterior and posterior views. Immediately after injection of BTAP-annexin $\mathrm{A} 5$ a dynamic study of 30 frames of 1 minute each was started. Subsequently, three whole body scans were performed using a scan speed of $10 \mathrm{~cm} / \mathrm{min}$. Typically these were acquired at $1 \mathrm{~h}, 4 \mathrm{~h}$ and $20 \mathrm{~h}$ post injection (p.i). 
Patients were not allowed to void before the first whole body scan. A transmission scan with a ${ }^{57} \mathrm{Co}$ flood source, with a strength decreasing from about $140 \mathrm{MBq}$ to $60 \mathrm{MBq}$ during the period of the dosimetric project, was obtained before the start of the BTAP. annexin $A 5$ study. This scan was used to estimate patient thickness that was needed for correction for body background activity and attenuation. Blood samples were taken just before administration of the activity, afterwards every 10 minutes during the first hour, and at about $4 \mathrm{~h}$ and $20 \mathrm{~h}$ p.i. In this study all feces and urine were collected during the first $20 \mathrm{~h}$. Activity in blood and urine was measured using a gamma counter (1282 Compugamma CS, LKB-Wallack, Turku, Finland), whereas the feces were measured with a gamma camera in the presence of a calibration source.

\section{Data analysis}

Regions of interest were drawn around all organs that showed noticeable uptake of ${ }^{99} \mathrm{~m}$ 'Tc activity, around the bladder, and around the calibration source that was used to check system stability. These same regions were used in all images, i.e. in the dynamic as well as the whole body scans.

Correction for body background activity was performed according to Buijs et al1 ${ }^{137}$. Finite organ thickness is taken into account in this approach. Organ thickness was derived from the organ volume as used in the MIRD methodology ${ }^{138}$ and the area of the region of interest. Absolute ${ }^{99 \mathrm{~m}} \mathrm{~T}$ 'c organ activity was estimated by correcting measured counts for scatter and attenuation according to a method published by Siegel et al ${ }^{139}$. Total body activity was determined using the "no excretion" approach, i.e. the ratio of the total number of counts in the first whole body scan, acquired before excretion, and the injected dose is taken as a counts to fraction of injected dose conversion factor ${ }^{140}$. Organ uptake was calculated as the activity in the organ corrected for physical decay, and is presented as a percentage of the injected dose (ID).

The absorbed dose in the red bone marrow was estimated from the activity found in the iliac crests, assuming that about $4.4 \%$ of the total amount of red marrow is present in those locations ${ }^{141}$.

In the images some overlap of activity in different organs frequently occurred, for instance, between activity in the right kidney and the liver. We tried to correct for this overlap by setting the activity in the right kidney equal to that in the left, unless the apparent activity in the right kidney was less than in the left kidney. The liver activicy, determined in a region that excluded the overlap with the right kidney, was augmented with the difference berween the right and left kidney, but only if the difference was positive. In most of the images acquired at $20 \mathrm{~h}$ p.i. there was also overlap between activity in the colon on the one hand, and activity in the liver, kidneys, and spleen on the other hand. The activity in liver, kidneys and spleen at this late time was calculated by extrapolating the activity in the overlap-free part of these organs to the total organ. The colon activity was taken as the activity present in the total colon region minus the activity (found by extrapolation as described above) in 
the overlapping parts of liver, kidneys and spleen.

The fraction of the tracer to be excreted in the feces can be obtained in the following way. Calling the decay corrected total activity that has left the body at the time of the last whole body scan fID ${ }_{\text {our" }}$, the activity in the feces $\mathrm{ID}_{\text {feces, }}$ and the activity still present in the colon $\mathrm{IID}_{\text {colon }}$, it is given by $\left(\mathrm{FD}_{\text {fees }}+\mathrm{fID}_{\text {colon }}\right) /\left(\mathrm{fID}_{\text {out }}+\mathrm{fID}\right.$ colon $)$. It is assumed that no re-uptake from the colon takes place. The callculated ratio only holds for the time interval during which excretion was monitored, since metabolisation of the tracer can result in a change in the relative contribution to the clearance by the liver and kidneys. Note that the simpler ratio $\mathrm{fDD}_{\text {feces }} / \mathrm{fID}_{\text {our }}$ is likely to be much more variable because people may or may not defecate within a $20 \mathrm{~h}$ interval.

Organ residence time was obtained by integrating the organ activity curve that was normalized with the injected activiry, but not corrected for physical decay. For the liver, kidneys and spleen the activity curves were constructed from both the dynamic and whole body scans. For all other organs only the whole body data could be used, because the dynamic study covered only a part of the trunk. In the integration the available data points were connected pair wise with an exponential function. The first and last functions were extrapolated to time zero and infinity, respectively.

Quantification of organ activity, residence time and biological half-life was performed with own software developed on the Siemens Icon computer platform (Siemens Gammasonics, Hoffman Estate, IL, USA). Organ doses and effective dose were calculated with the Mirdose 3.1 program ${ }^{138}$, applying the dynamic bladder model ${ }^{142}$, with a voiding interval of $4 \mathrm{~h}$ and the individually measured biological half-life, 


\subsection{Results}

The injected dose of BTAP-annexin A5 was on average $579 \pm 89 \mathrm{MBq}$; the radiochemical purity was $96 \pm 4 \%$.

Figure 4.1 shows a typical example of the activity distribution at $1 \mathrm{~h}, 4 \mathrm{~h}$ and $20 \mathrm{~h}$ p.i. Uprake in target regions, i.e. regions where apoptosis might have been increased due to disease, was negligible from a dosimetric point of view. Since all patients had normal kidney and liver function, it was assumed that all data could be pooled and considered as representative for normal humans.

In Figure 4.2 the average uptake and uptake \pm the standard deviation are shown as a function of time for a few source organs, including the left kidney, liver and spleen. A curve giving the activity in the total body is shown also. To enable comparison between BTAP-annexin A5 and the previously used Imino-annexin A5, the curve for the activity in the total body for the latter tracer agent has been included.

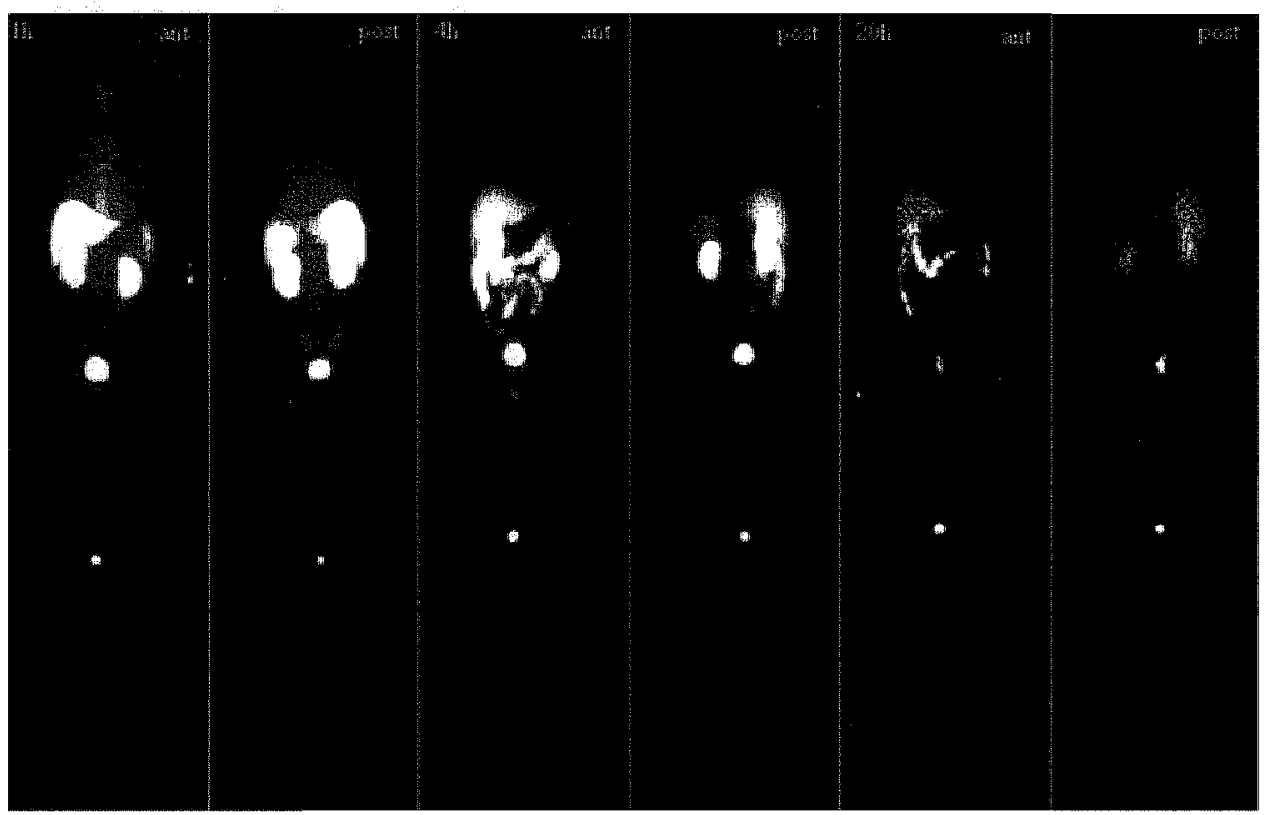

Figure 4.1 Conjugate views from whole body scans of a male ( $53 \mathrm{y})$, acquired at about $1 \mathrm{~h}, 4 \mathrm{~h}$ and $20 \mathrm{~h}$ after intravenous injection of $566 \mathrm{MBq}$ of BTAP-annexin A5. In each pair at the left the anterior and at the right the posterior view. 

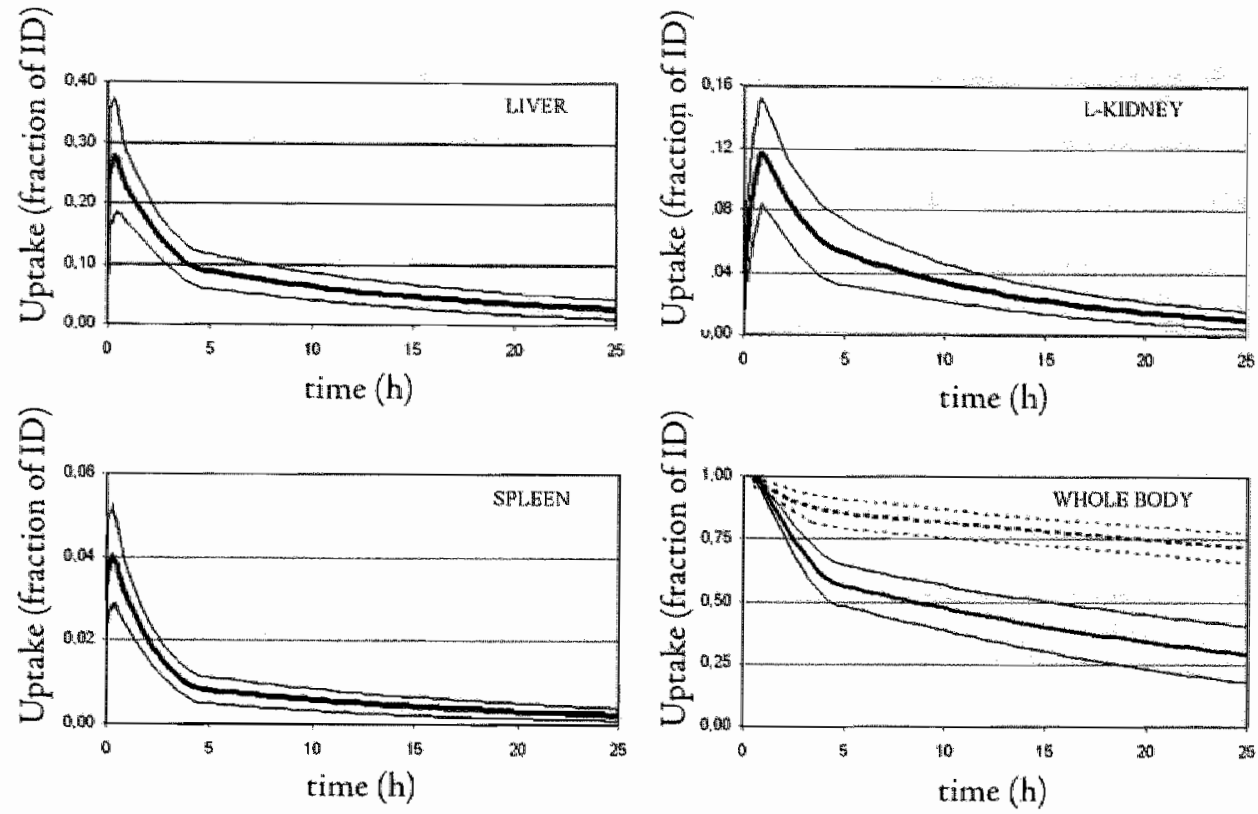

Figure 4.2 Decay corrected time - $9 \%$ wc uptake curves for liver, left kidney, spleen and total body after intravenous injection of BTAP-annexin A5. Curves with mean and [mean \pm 1 standard deviation] are shown. Fot comparison, the totall body curves for Imino-annexin A5 are shown also (dashed lines).

The effective biological half-life in the total body is $16 \pm 7 \mathrm{~h} \mathrm{BTAP-annexin} \mathrm{A5}$, and $62 \pm 13 \mathrm{~h}$ for Imino-annexin A5. It is noted that this biological half-life has been estimated by fitting a single exponential function to the (three) data points obtained from the whole body scans. This will yield some effective value, because the clearance is initially faster than at later times, as can be seen from Figure 4.2 (after the wash-in period). Pharmacokinetic parameters of decay corrected $\% \mathrm{mc}$ activity in blood are given in 'Table 4.1 .

Table 4.1: Pharmacokinetic parameters of decay cortected "mo activity in blood during the first 20 hours after injection in patients $(n=8)$

\begin{tabular}{lllll}
\hline Component & $\alpha$ & & \multicolumn{3}{c}{$B$} & \\
\hline Measurement & $t^{1 / 2}(\min )$ & Fraction & $t^{1 / 2}(\mathrm{~h})$ & Fraction \\
& $26 \pm 5$ & $0.87 \pm 0.04$ & $6.9 \pm 1.4$ & $0.13 \pm 0.04$ \\
\hline
\end{tabular}


The data could reasonably well be fitted with a two-component model. It should be noted, however, that the resulting parameters depend on the time interval used in the fit (here $0-20 \mathrm{~h}$ ). This shows that a two-component model is not really appropriate, but that more components or a distribution of components must exist.

Table 4.2 presents the average uptake for all source organs at $70 \mathrm{~min}$ after intravenous injection. We grouped organ uptake values in three categories, (non-) Hodgkin $(n=3)$, decompensatio cordis $(n=3)$ and subacute infarction $(n=2)$ and compared these groups using a one-way ANOVA model. Applying a significance level of 0.05 with a Bonferroni-Holmes correction, the inter-group differences were found to be statistically not significant. Effective half-life, tesidence time and absorbed dose for a number of organs have been included also in Table 4.2. For comparison the corresponding absorbed doses for the previously studied Imino-annexin A5 are shown as well ${ }^{87}$. It is seen that with both formulations the kidneys receive the highest absorbed dose $(63 \mu \mathrm{G} y / \mathrm{MBq}$ for BTAP-annexin A5, compared to $93 \mu \mathrm{Gy} / \mathrm{MBq}$ for Imino-annexin A5). Other organ absorbed doses are at least a factor 3 lower. The effective dose for BTAP-annexin $A 5(7.6 \pm 0.5 \mu \mathrm{Sv} / \mathrm{MBq})$ is lower than that for Imino-annexin $\mathrm{A} 5(9.7 \pm 1.0 \mu \mathrm{Sv} / \mathrm{MBq})$.

At $20 \mathrm{~h}$ p.i. the total fraction excreted was $65 \pm 11 \%$ of the ID, while the fraction found in the feces was $7 \pm 8 \%$ of the ID. The activity excreted in the urine up to $20 \mathrm{~h}$ p.i. can be estimated from measurements on the collected urine, which yields $57 \pm$ $12 \%$ of the ID, but also from the difference between the total activity that has left the body and that present in the feces, which yields $58 \pm 9 \%$. The fraction of the injected dose to be excreted in the feces, estimated from measurements up to $20 \mathrm{~h}$ p.i., was 27 $\pm 6 \%$; the rest will be excreted in the urine. 
Table 4.2: Dosimetric data (mean \pm SD) for intravenously administered BTAP-annexin A5 $(n=8)$

\begin{tabular}{|c|c|c|c|c|c|}
\hline & $\begin{array}{l}\text { uptake at } \\
70 \text { min p.i." }\end{array}$ & $\begin{array}{l}\text { effective } \\
\text { half } \\
\text { life" }\end{array}$ & $\begin{array}{l}\text { residence } \\
\text { time }\end{array}$ & $\begin{array}{l}\text { absorbed } \\
\text { dose }\end{array}$ & $\begin{array}{l}\text { absorbed dose } \\
\text { Tc-1 AnxA5 }\end{array}$ \\
\hline Organ & {$[\% \mathrm{LD}]$} & {$[\mathrm{h}]$} & {$[\mathrm{h}]$} & {$[\mu \mathrm{Gy} / \mathrm{MBq}]$} & {$[\mu \mathrm{G} \gamma / \mathrm{MBq}]$} \\
\hline Kidneys & $27.7 \pm 8.0^{\circ}$ & $3.2 \pm 0.5$ & $1.20 \pm 0.45$ & $63 \pm 22$ & $93 \pm 24$ \\
\hline Liver & $20.2 \pm 4.4$ & $3.5 \pm 0.5$ & $0.85 \pm 0.18$ & $13 \pm 3$ & $17 \pm 2$ \\
\hline Red bone marrow & $4.6 \pm 2.2$ & $3.8 \pm 0.7$ & $0.22 \pm 0.10$ & $4.0 \pm 0.7$ & $5.5 \pm 0.8$ \\
\hline Spleen & $2.8 \pm 0.8$ & $3.4 \pm 0.4$ & $0.12 \pm 0.03$ & $15 \pm 3$ & $22 \pm 6$ \\
\hline Testes $(n=6)$ & $0.24 \pm 0.08$ & $3.7 \pm 0.7$ & $0.015 \pm 0.005$ & $6.3 \pm 1.6$ & $15 \pm 3$ \\
\hline Thyroid & $0.28 \pm 0.12$ & $3.2 \pm 0.4$ & $0.010 \pm 0.005$ & $5.6 \pm 3.6$ & $10 \pm 6$ \\
\hline Urinary bladder & - & - & $0.39 \pm 0.09$ & $20 \pm 6$ & $7.5 \pm 2.6$ \\
\hline Remaindler & - & - & $2.50 \pm 0.69$ & - & - \\
\hline Total body & $96.7 \pm 1.9$ & $4.2 \pm 0.4$ & $5.65 \pm 0.49$ & $7.6 \pm 0.5^{a}$ & $9.7 \pm 1.0^{30}$ \\
\hline
\end{tabular}

\section{Notes:}

"Uptake values are corrected for physical decay.

${ }^{t}$ Results are from a fit with a single exponential.

* From reference ${ }^{87}$

'This is two times left kidney uptake; see rext.

Dynamic bladder model ${ }^{42}$; voiding interval $4 \mathrm{~h}$, biological half-life of activity in total body from individual measurements (average $16 \pm 7 \mathrm{~h}$ ), fraction of activity to urine $=0.7$.

\& Effective dose (ICRP 60) in $\mu \mathrm{S} v / \mathrm{MBq}$. 


\subsection{Discussion}

Application of BTAP-annexin A5 in patients causes an effective dose of $7.6 \pm 0.5$ $\mu \mathrm{Sv} / \mathrm{MBq}$, corresponding to a total effective dose of $4.6 \pm 0.3 \mathrm{mSv}$ for a typical patient dose of $600 \mathrm{MBq}$. These values are slightly lower $(22 \%)$ than those for the previously studied compound Imino-annexin $\mathrm{A} 5^{87}$. Although this reflects that most organs received a lower absorbed dose, some otgan doses are increased. For instance, the absorbed dose to the urinary bladder and the upper and lower large intestine wall are approximately a factor of $2-3$ higher. The effective biological half-life in the total body is $16 \pm 7 \mathrm{~h}$ for BTAP-annexin A5 versus $62 \pm 13 \mathrm{~h}$ for Imino-annexin A5. Therefore, the differences in organ and effective doses are probably the result of the faster clearance of BTAP-annexin A5, as this is beneficial for most organs, except the bladder and the gut.

Our inclusion of patients with various diseases in this study may need some discussion. Clearance of the tracer is mainly effected by the kidneys and the liver, and the function of these organs was normal in all patients. The patients differed from normal persons only in some focal disease, which could cause a local increase in apoptosis. The uptake due to this apoptosis is but a very small fraction of the injected dose, and dosimetrically hardly relevant. On the other hand, there was a considerable variability in uptake and effective half-life of activity, e,g. in the liver and the kidneys, as may be seen from the standard deviations in Table 4.2. Because we studied a rather limited number of patients, the finding that our data did not support the hypothesis that this variation is associated with the disease may not yet be conclusive. However, even if the variability is disease related, the impact of these variations on the effective dose will be small, as the effective dose is rather insensitive to the distribution of the acrivity. "This can be seen from its small relative standard deviarion of less than $7 \%$, even when organ uptakes vary by as much as $30 \%$.

In the previous study the high uptake of Imino-anmexin A5 in the kidneys and the liver was expected not to allow the study of apoptosis in these organs and their immediate neighbourhood. The uptake of BTAP-annexin A5 in the kidneys and liver is even slightly higher, and, in addicion, activity is excreted considerably faster to the gut. The fractions excreted in the urine and in the feces (including those still in the colon) were similar, however: $0.27 \pm 0.06$ for BTAP-annexin A5 versus $\approx 0.25$ for Imino-annexin $A 5^{87}$, where both figures were calculated over an interval of about $20 \mathrm{~h}$ The faster excretion of BTAP-annexin A5 may well preclude its use in the whole abdomen. On the other hand, the study of the thorax and the head/neck region seems well feasible. It is to be noted that annexin A5 will not cross the intact blood brain barrier, while the behavior of annexin $A 5$ in brain tumors and brain infarctions inparients still has to be elucidated.

The presently investigated compound BTAP-annexin A5 has the advantage of a higher radiochemical purity $(96 \pm 4 \%)$ than Imino-annexin A5 $(82 \pm 12 \%)$, but 
its elaborate preparation is not very suitable for common clinical application. New conjugation methods to obtain 99 "Tc-annexin A5 with better inaging properties, like HYNIC-annexin A5, are currently under investigation ${ }^{65}$.

The best time for imaging may depend upon the application. In our experience, when studying patients with myocardial infarction, the best results were obtained somewhere between 4 and 12 hours after administration. From an imaging point of view, the long biological half-life of BTAP-annexin A5 $(16 \mathrm{~h})$, more than twice the physical half-life of ${ }^{99 m} T c(6 \mathrm{~h})$, is certainly still not optimal.

As a kind of check on our red bone martow dosimetry we made also estimates from activity in the blood, assuming no binding of activity in the marrow, according to a method published by Shen et al ${ }^{143}$. Using their approach one finds an estimated absorbed dose of $1.4 \pm 0.3 \mathrm{mG}$ for the average injected activity of $580 \mathrm{MBq}$, while the presently used method, which is based on regions a round the iliac crests ${ }^{141}$, gives $2.3 \pm 0.4 \mathrm{mGy}$. The difference can probably be explained by some binding in the martow, as suggested before ${ }^{87}$. 


\subsection{Conclusion}

The biodistribution and dosimetry of BTAP-annexin A5, a radioactive tracer agent intended for the in vivo study of apoptosis, has been investigated. The main excretion routes are via the kidneys and the liver. The compound has a biological half-life of about $16 \mathrm{~h}$, which is long compared to the physical half-life of ${ }^{99 \mathrm{~m}} \mathrm{Tc}(6 \mathrm{~h})$.

The strong accumulation of this compound in the kidneys, liver and gut may cause serious problems in studying apoptosis in the abdomen. The effective dose of $7.6 \pm 0.5$ $\mu \mathrm{Sv} / \mathrm{MBq}$ associated with the application of BTAP-annexin A5 is in the lower range of values found for commonly used ${ }^{99 m}{ }^{9} \mathrm{Tc}$ compounds ${ }^{144}$. From a dosimetric point of view this makes BTAP-annexin A5 even better suited for studies of apoprosis in patients than the formerly used Imino-annexin A5. This is certainly of value when changes in apoptosis are to be monitored in repeated studies in the same patient. 



$$
5
$$




\section{Minocycline inhibits cell death in vivo in myocardial ischemia and subsequent reperfusion}

Adapted from: H.H. Boerstma, A.D. Petrov, S. L. Wolters, N. Bitsch, M. Sarai, S. Isobe, A. Bennaghmonch, N. Haider, B.L. Kietselaer, L.M. Stolk, G.JJ. Teule, G.A.K. Heidendal, L.Hofstra, C.P.M. Reutelingsperger,J.Narula. Minocycline inbibits cell death in viwo in myocardial ischemia and subsequent reperfusion. Submitted. 


\subsection{Introduction}

Myocardial infarction is a commonly occurring disease caused by acute thrombotic occlusion of coronary arteries in the heart. The latrer starts initially with ischemia, resulting in myocardial oxygen and glucose depletion. The lack of oxygen results in acute myocardial metabolic acidosis, lowering the $\mathrm{pH}$ of the heart from 7.2 to 6.5. When accurate medical trearment is applied, thrombus dissolution is obtained, which results in reperfusion of the ischemic myocardium. Myocardial reperfusion has been shown to be a strong trigger for programmed cell death ${ }^{100.145-149}$. It causes oxidative stress by generation of reactive oxygen species (ROS), which are believed to trigger apoptotic stimuli. ROS alter the mitochondria, which predominantly enable the release of cytochrome $C$ and AIF, respectively. The latter events are important triggers for the induction of apoptosis ${ }^{150}$. Other key players in the cell death program include caspases ${ }^{151.153}$, and members of the $\mathrm{Bcl}-2$ family ${ }^{153-155}$ and constitute attractive targets for interventional therapies. Caspase 3 is considered to be the executioner caspase, the activation of which marks the point of no return ${ }^{79}$. Caspase 3 activation triggers PS expression at the cell membrane surface ${ }^{80,81}$. This process is one of the early events of apoptosis and persists until the cell is destroyed ${ }^{59}$. The pivotal role played by caspases in the propagation of the apoptotic process make the individual family members an attractive rarget for pharmacological intervention ${ }^{156}$.

Caspases can be targeted directly with caspase specific inhibitors or indirectly by interfering with the mechanisms resulting in the activation of caspases ${ }^{156}$.

Minocycline is a tetracycline with antibiotic and anti-inflammatory properties, which is used clinically for the treatment of diseases such as urethritis and rheumatoid arthritis ${ }^{157,158}$. Experimentally, minocycline has also been shown to be neuroprotective in animal models of cerebral ischemia and to delay progression and improve survival in mouse models of neurodegenerative diseases ${ }^{159-161}$. Furthermore, in vitro, it has been shown that minocycline exerts cardioprotective properties in myocardial ischemia and reperfusion models. These anti-apoptotic effects were subscribed in part to the caspase inhibiting capacities of minocycline $e^{156,162}$.

Here we investigated the effect of minocycline on cardiomyocytic apoptosis using two animal models utilizing 30-40 minutes of myocardial ischemia followed by 1 or 3 hours of reperfusion, using $99 \mathrm{~m}$ Tc-annexin A5 and Alexa568-annexin A5 to measure apoptosis. ${ }^{99} \mathrm{~T} \mathrm{~T} c$-annexin $\mathrm{A} 5$ was tested as a novel method for quantification of myocardial infarction, whereas the fuorescent Alexa568-annexin A5 was used as a standard method for apoprosis of evaluation ${ }^{62}$. The animal experiments were approved by the respective animal experiment ethics committees of the Maastricht Univetsity (mouse experiments) or the University of California, Irvine (rabbit experiments). 


\subsection{Materials and Methods}

\section{Radiolabeling of HYNIC-annexin A5}

Recombinant human annexin A5 was used to prepare the used radiopharmaceutical. ${ }^{99}$ Tc-HYNIC-annexin A5 (Theseus Imaging Corp., Cambridge MA) was used to perform $\mathrm{I} / \mathrm{R}$ experiments in rabbits ${ }^{65}$. For each experiment, a vial containing 100 $\mu \mathrm{g}$ of human recombinant annexin A5 conjugated with $\mathrm{HYNIC}^{65}$ was thawed after storage (at $-20^{\circ} \mathrm{C}$ ) and mixed with ca. $740 \mathrm{MBq}$ pertechnetate $(1 \mathrm{ml}$ ). Subsequently, $0.1 \mathrm{ml}$ stannous tricine buffer was added to the vial for pertechnetate reduction. This mixture was allowed to incubate ( $30 \mathrm{~min}, \mathrm{r.t.}$.).

The $\mathrm{pH}$ was measured (limits $\mathrm{pH}$ 5-7) and RCP was assessed by TLC on silica impregnated paper (Gelman Sciences, Ann Arbor, MI) using ACD buffer as the eluen $t^{89}$. HYNIC-annexin $A 5$ remains at the position of application ( $\left.R f=0\right)$, whereas most radioactive impurities are at the solvent front $(\mathrm{R} f=1)^{89}$.

\section{$I / R$ of the murine beart in vivo}

Two month old Swiss mice (Charles River) were anesthezised with pentobarbital (100 $\mathrm{mg} / \mathrm{kg}$ i.p.), and the trachea was intubated perorally with a stainless steel tube. The animals were mechanically ventilated with room air. After left thoracotomy and exposure of the heart the left anterior descending coronary attery (LAD) was ligated with 6-0 polypropylene just proximal to its main branching point. The surture was tied over a $1-\mathrm{mm}$ polyethylene tube (PE-10) that was left in place during the 30 min period of ischemia. Blood flow was then reestablished by removal of the tube. The occurrence of reperfusion could be assessed by the observation of blood flow in epicardial coronary arteries through the operation microscope. After this procedure, the animals were available for imaging using fluorescently labelled Alexa568-annexin A5. A subgroup $(\mathrm{n}=3)$ received a single $30 \mathrm{mg} / \mathrm{kg}$ intravenous bolus of minocycline (Sigma St. Louis, MO). Minocycline was dissolved in saline with the aid of DMSO prior to injection into the animals. The controls $(n=3)$ remained untreated.

In vivo imaging with Alexa568-annexin A5 in mice

Surgery and imaging was performed using a stereomicroscope (Leica MZ FL III, Leica, Switzerland). During surgery and the imaging procedure, the mouse body temperature was maintained at $36.5^{\circ} \mathrm{C}$ and electrocardiogram (ECG) was monitored. Selective excitation of Alexa-568 was produced through a $568-\mathrm{nm}$ band-pass filter. Emitted fluorescence was collected through a band-pass filter of $600 / 50 \mathrm{~nm}$ on a Hamamatsu C4742-95 cooled charge-coupled-device camera (CCD) (Hamamatsu Photonics Systems, Hamamatsu City, Japan), which was triggered by the ECG in diastole. Images were processed and analysed with the use of Imagejb software 
and Microsoft PhotoDraw. An arterial line (polyethylene, PE-10) was introduced into the left carotid artery for injection. Minocycline was injected upon myocardial reperfusion. Alexa568-annexin A5 $(2.5 \mathrm{mg} / \mathrm{kg}$. Nexins Research, Kattendijke, The Netherlands) was injected $2 \mathrm{~min}$ after the onset of reperfusion. The heart was exposed through thoracotomy. During the experimental procedure, the exposed area of the heart was protected from temperature changes and drying with a controlled moisture system. This imaging protocol allowed capturing images up to $1 \mathrm{~h}$ after reperfusion.

\section{$I / R$ of the rabbit beart in vivo}

New Zealand White Male Rabbirs (2.5 to $3.0 \mathrm{~kg}$, Western Oregon; Philomath, OR) were anesthezised with $2.0-3.0 \%$ isoflurane. Surgical tracheostomy was performed, and ventilation was maintained with a Harvard rodent positive-pressure respirator. The heart was exposed through parasternal thoracotomy, and the pericardium was removed. The region of the LAD was identified between the aortic root and the left auricle, and a monofilament suture was placed at the site. The LAD was occluded by tightening the snare created by passing the suture through a polyethylene tube. The snare was removed from the LAD after 40 minutes of occlusion ${ }^{163}$. The occurrence of reperfusion could be assessed by the observation of blood flow in epicardial coronary arteries. After 30 min of reperfusion, HYNIC-annexin A5 (370 $29 \mathrm{MBq})$, was administered. A subgroup of 3 animals received then a single $30 \mathrm{mg} / \mathrm{kg}$ intravenous bolus of minocycline. Minocycline was dissolved in saline prior to injection into the animals. The other 3 animals were untreated. Animals remained under anesthesia until $3,5 \mathrm{~h}$ after reperfusion and were then sacrificed using an overdose of sodium pentobarbital $(120 \mathrm{mg} / \mathrm{kg})$.

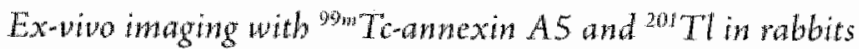

For apoptosis imaging, "Mm Tc-annexin A5 was injected intravenously through the marginal ear vein. Blood samples were withdrawn from the femoral artery at 1,5 , $15,30,60,120$ and 180 minutes after intravenous administration of the radioisotope in control animals, for blood clearance studies. The pharmacokinetic parameters were derived using the KINFIT module of the MW/PHARM computer program package (Version 3.30, MediWare, Groningen, The Netherlands) ${ }^{134}$.

To assess the area at risk, 3 min before the sacrifice of each animal ${ }^{201} \mathrm{Tl}(8.9 \pm$ $2.5 \mathrm{MBq}$ ) was administered intravenously. The heart was carefully dissected. Therafter, perfusion imaging and imaging of cell death was done simultaneously using a dual isotope protocol. Radionuclide imaging was performed with a twohead micro-SPEC'T gamma camera with micro-CT (X-SPECT, Gamma Medica, Inc; Northridge, CA). SPECT images of the heart were acquired in $64 \times 64$ scaffold, 32 steps at 120 seconds per step on $140 \mathrm{keV}$ photopeak of ${ }^{99 \mathrm{~m}} \mathrm{Tc}$ with $15 \%$ 
window using low energy parallel-hole collimator for ${ }^{9 \%}$ Tc images, combined and reconstructed. Images were captured for $0.5 \mathrm{~s}$ per view for 256 views in $360^{\circ}$ rotation. After SPECT acquisition, a micro-CT image was acquired. The micro-CT used an $X$-ray tube operating at $50 \mathrm{kV}$ and $0.8 \mathrm{~mA}$. Images were captured for $0.5 \mathrm{~s}$ per view for 256 views in $360^{\circ}$ rotation. The micro-SPECT images were converted to $256 \times 256$ scaffold and micro-CT tomographic studies were fused using the X-SPECT fusion computer programme (X-SPECT, Gamma Medica, Inc; Northridge, CA), allowing the achievement of simultaneous scintigraphic and anatomical information in all tomographic scans in thee different spatial axes. Furthermore, planar images of the ex-vivo heart were obtained for 15 min in a $128 \times 128$ matrix using a 1 mn low energy pin-hole collimator. Thereafter the rabbit beart was cut into 4 slices (Figure 5.4). For quantitarive uptake data, each slice was further divided into 8 pieces. Radioactivity in the pieces of the heart was determined with gamma scintillation counting ( 1480 Wizard $3 "$; Wallac Co.). In the case of the administration of "99m-annexin A5, an automatic spill correction program for ${ }^{201} \mathrm{Tl}$ contamination was applied as well as by serial assessment of $99 \mathrm{~m}$ T was performed. For the quantitative assessment of annexin uptake, the uptake (percent injected dose per" gram) pieces from the infarct territory and uptake pieces from the remote myocardial region were picked for the assessment of distribution of HYNIC-annexin A5 activity within each animal heart. A value of $\% \mathrm{ID} / \mathrm{g}>0.3$ was considered as high uptake within the infarcted area of each myocardium.

To assess biodistribution of HYNIC-annexin A5 in the tabbit, organ samples of lung, liver, spleen, cortex, medulla, small intestine, large intestine, stomach, muscle, bladder, testis, urine and bone martow were excised, weighed and counted in the same way as decribed for the heart.

\section{Stakistical evaluation}

Dara were statistically evaluated using Student's t-test. A probability value $<0.05$ was considered significant. Results are presented as means $t$ standard deviation, unless otherwise specified. 


\subsection{Results}

In vivo imaging in mice with Alexa568-annexin A5: the elfect of minocycline Annexin A5 imaging is a powerful tool to assess reperfusion damage in the heart after an ischemic episode. Using Alexa568-annexin A5, cell death was detected in mice undergoing $30 \mathrm{~min}$ ischemia and subsequently $1 \mathrm{~h}$ of reperfusion. Figure 5.1 (upper panel) shows an example of the ex-vivo uptake in the untreated heart, Figure 5.1. (lower panel) shows the uptake in the minocycline $(30 \mathrm{mg} / \mathrm{kg})$ treated heart. In vivo uptake area values after 60 min were determined to be $7.66 \times 10^{4} \pm 2.03 \times 10^{4}$ for minocycline treated animals and $2.04 \times 10^{5} \pm 1.87 \times 10^{4}$ for the untreated controls. After $1 \mathrm{~h}$ of reperfusion, the uptake in the treated hearts was significantly lower (ca. $60 \%)$ than in the untreated controls $(p<0.005)$. An impression of the time-course of Alexa568-annexin A5 kinetics can be observed in Figure 5.2 After the initial bloodpool-phase, Alexa568-annexin A5 is either taken up by apoptotic myocardial cells or washed our from the tissue by the regular circulation.

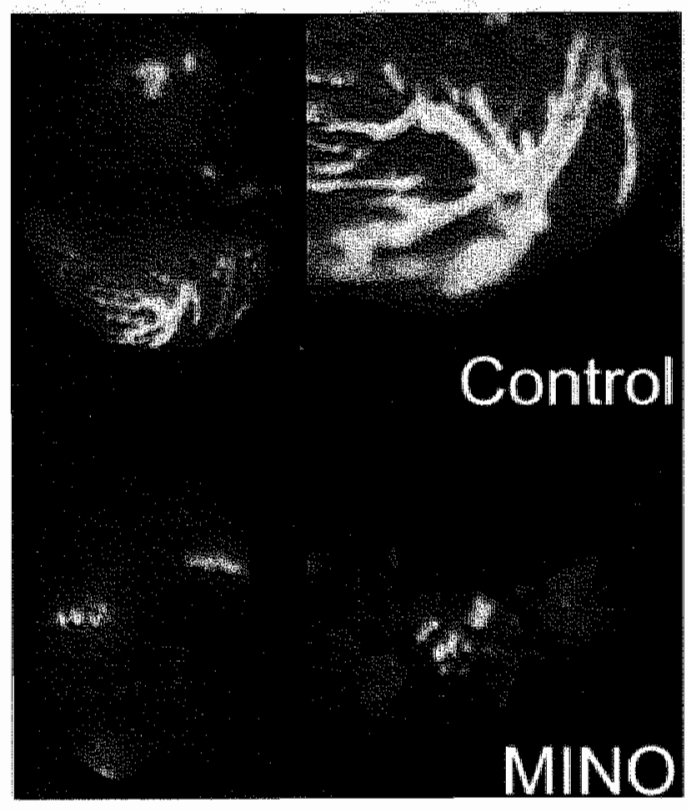

Figure 5.1: Ex-vivo images of Alexa568-annexin A5 uptake in examples of a mouse heart after $I / R$ and $1 \mathrm{~h}$ of reperfusion. The left side of the figure shows overviews on the whole heart (magnitude: 2.0 ). The righe side gives a detailed view of uptake in the hearts at the cellular level (magnitude 10.0). The upper panel depicts the untreated Control, whereas the lower panel (MINO) shows the results of treatment upon reperfusion with minocycline ( $30 \mathrm{mg} / \mathrm{kg}$ ), respectively. The lower annexin A5 uptake in the treated hearts is indicative of inhibition of cell death under these treatment circumstances. 


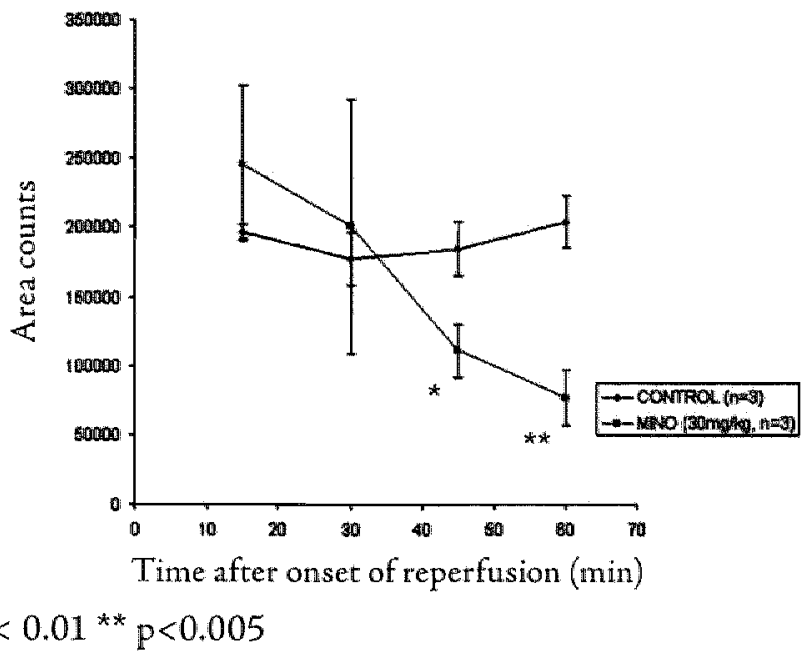

Figure 5.2* Time course of Alexa 568-annexin A5 uptake (expressed as area counts) after onset of reperfusion in the mouse heart. Uptake area counts are depicted versus time (min). The initial high uptake is due to the appearance of the injected Alexa568-annexin $A 5$ in the blood. After clearance of Alexa568-annexin A5 from blood, it is bound to the cardionyocytes. After 45 and 60 min of reperfusion, Alexa568-annexin A5 binding to minocycline (MINO) treated animals is significantly lower compared to untreated CONTROL mice.

\section{Annexin A5 imaging in rabbits: treatment with minocydine}

In order to be able to perform more accurate quantifications after cell death assessment we shifted to an $I / R$ model using tabbits instead of mice. Moreover, this was done because we wanted to walidate a nuclear imaging method. After 40 min of ischemia and $3 \mathrm{~h}$ of subsequent reperfusion, the rabbit hearts were subjected to exvivo imaging. Enhanced localization of $99 \mathrm{mc}$-annexin $\mathrm{A} 5$ was observed in the infarct area (see Figure 5.3). Comparison of the images of minocycline treated animals with those of the untreated controls already shows a clearly lower $99 \mathrm{~m}$ Tcannexin A5 uptake in minocycline treated rabbits (Figure 5.3).

The specific uptake within the infarcted area in \%ID/g was evaluated for minocycline treated rabbits as well as for the untreated animals. High uptake was defined as $\% \mathrm{ID} / \mathrm{g}>0.3$. The results are shown in Table 5.1. Minocycline treated rabbits showed a significant $(p<0.02)$ reduction of $42 \%$ in uptake $(>0.3 \% \mathrm{ID} / \mathrm{g})$ of ${ }^{99 \mathrm{~m}} \mathrm{~T}$ c-annexin $\mathrm{A} 5$ in the area at risk of the heart as compared to control animals. The myocardial area at risk as assessed by ${ }^{201}$ Tl tended to be larger in the control groups, but was not significantly different from the minocycline treated group $(\mathrm{p}=\mathrm{NS})$. To get a semi-quantitative view and to improve the evaluability of the results, the $\% \mathrm{ID} / \mathrm{g}$ was calculated for $\% 9 \mathrm{~m} T \mathrm{c}$ and ${ }^{201} \mathrm{Tl}$. The ${ }^{99 \mathrm{~m}} \mathrm{Tc} /{ }^{201} \mathrm{Tl}$ ratio was plotted in a diagram (Figure 5.4). 
Table 5.1: The effect of minocycline on myocardial cell death during $40 \mathrm{~min}$ of ischemia and subsequent reperfusion, determined by ${ }^{9 m}$ TCHYNIC-annexin A5.

\begin{tabular}{|llll|}
\hline & No treatment & Minocycline-treatment & P-value \\
\hline $\begin{array}{l}\text { High uptake }(>0.3 \% \mathrm{ID} / \mathrm{g} ; \\
\mathrm{n}=3) \text { average } \% \mathrm{ID} / \mathrm{g}\end{array}$ & $0.65 \pm 0.10 \%$ & $0.38 \pm 0.07 \%$ & $\mathrm{p}_{<0.02}$ \\
\hline
\end{tabular}

Each ring in the diagram represents a slice in the heart. The inner circle depicts the apex, and the other rings represent slices lying above the apex. The diagrams represent the result of a representative minocycline treated animal as well as an untreated rabbit. These results sustain the other presentation forms of the data. The untreated animal predominantly shows higher ${ }^{99} \mathrm{rm} /{ }^{201} \mathrm{Tl}$ ratio's than minocycline treated animals (Figure 5.4).

\section{A: No treatment}
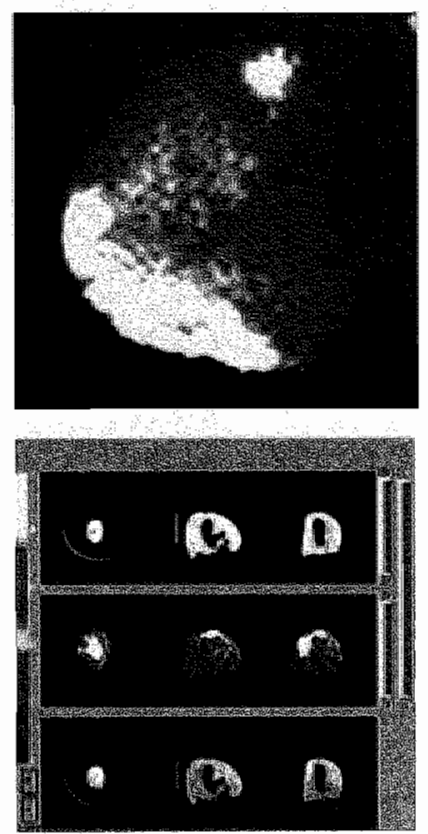

\section{B: Minocycline-treated}
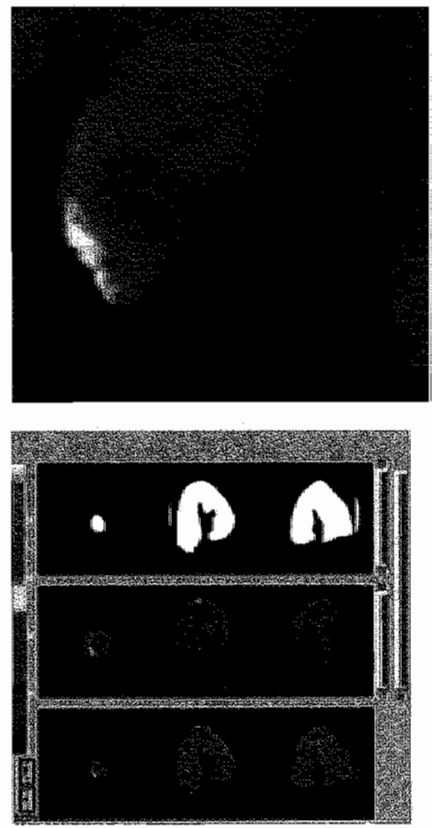

Figure 5.3 (upper panel) A: HYNIC-annexin A5 uptake in a representative example of a rabbit heart without minocycline adninistration upon reperfusion. B: HYNIC-annexin A5 uptake after treatment with minocycline $(30 \mathrm{mg} / \mathrm{kg}$ ). (lower panel) Ex vivo annexin A5 SPEC I-CT fusion images, which show in detail the anatomical location of the annexin A5 uptake in the rabbit myocardium. It is evident that both infarctions were apical. Image A shows the control animal, whereas Image $B$ depicts the minocycline treated animal. 


\section{A: No treatment}

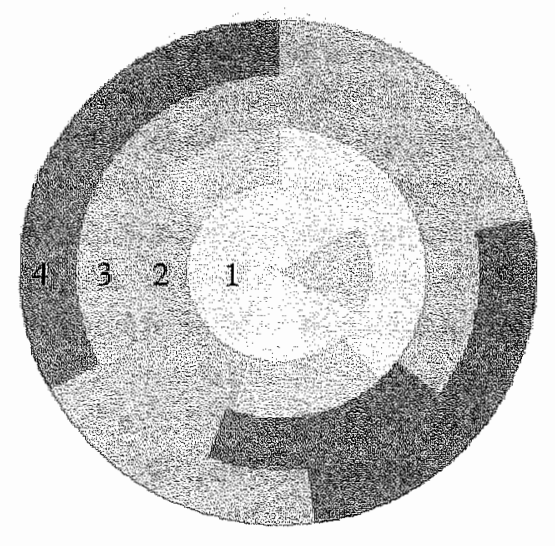

\section{C: Model of the rabbit heart}

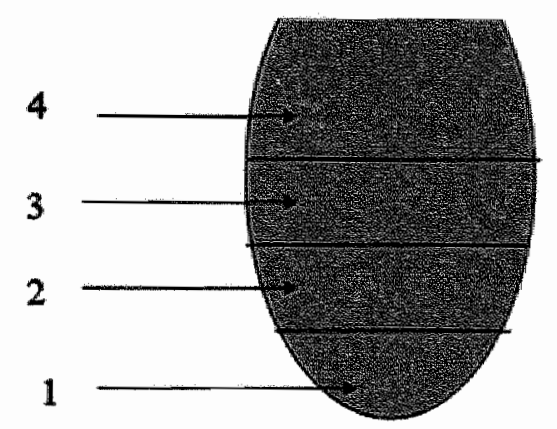

Legend: ${ }^{99 n^{2}}$ Tc/ ${ }^{20 !}$ TI uptake rario.

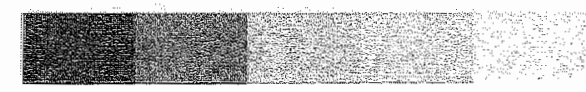

\section{$\begin{array}{llllll}0-1 & 1-2.5 & 2.5-5 & 5-10 & 10-20 & >20\end{array}$}

Figure 5.4: Upper: Circle diagrams display the uptake ratio of HYNIC-annexin A5/ X TI in two individual rabbits. The observed lower ${ }^{99 \mathrm{~m}} \mathrm{Tc} /{ }^{201} \mathrm{Tl}$ ratios minocycline-treated animals clearly represent the reduction of cell death within the area at risk of these hearts.

Lower: Model of the rabbit heart showing the slices, which were cut to quantify uptake of radioactivity in the heart. The numbers in the circle diagrams correspond to the numbers in the heart model. Furthermore, the analyzed pieces were arranged in an anatomically correct way. 
Pharmacokinetics/Biodistribution of ${ }^{99 n}$ TC-HYNIC-annexin A5 in rabbits Radiolabeling of HYNIC-annexin A5 yielded a radiochemical purity of $91.3 \pm 4.3$ $\%$. No side effects were observed after the administration of the radiopharmaceuticals or treatment agents to the animals. The kinetics of HYNIC-annexin A5 could satisfactorily be described by a two-compartment model. Figure 5.5 shows the biodistribution to the main organs of HYNIC-annexin $A 5$ in rabbits after $I / R$, with and without minocycline. Minocycline treated animals showed a statistically significant $(p<0.05)$ reduction in the uptake of ${ }^{99}$ Tc-annexin A5 by the lung and kidney medulla. Table 5.2 shows the main pharmacokinetic parameters obtained for the blood clearance of ${ }^{99 \mathrm{~m}}$ Tc-annexin A5.

Table 5.2: Pharmacokinetic paramerers of HYNIC-annexin A5. As the values of untreated and minocycline treated animals were comparable, these were taken together.

\begin{tabular}{|c|c|c|c|c|}
\hline Parameter $\rightarrow$ & $\mathbf{t}_{s, i x}$ & $t_{5,8}($ range, median $)$ & $V_{\text {rot }}$ & $\mathrm{Cl}$ \\
\hline & $15.5 \pm 9.3 \mathrm{~min}$ & $2.0-187 \mathrm{~h}(11.0 \mathrm{~h})$ & $886 \pm 5341$ & $93 \pm 821 / h$ \\
\hline
\end{tabular}

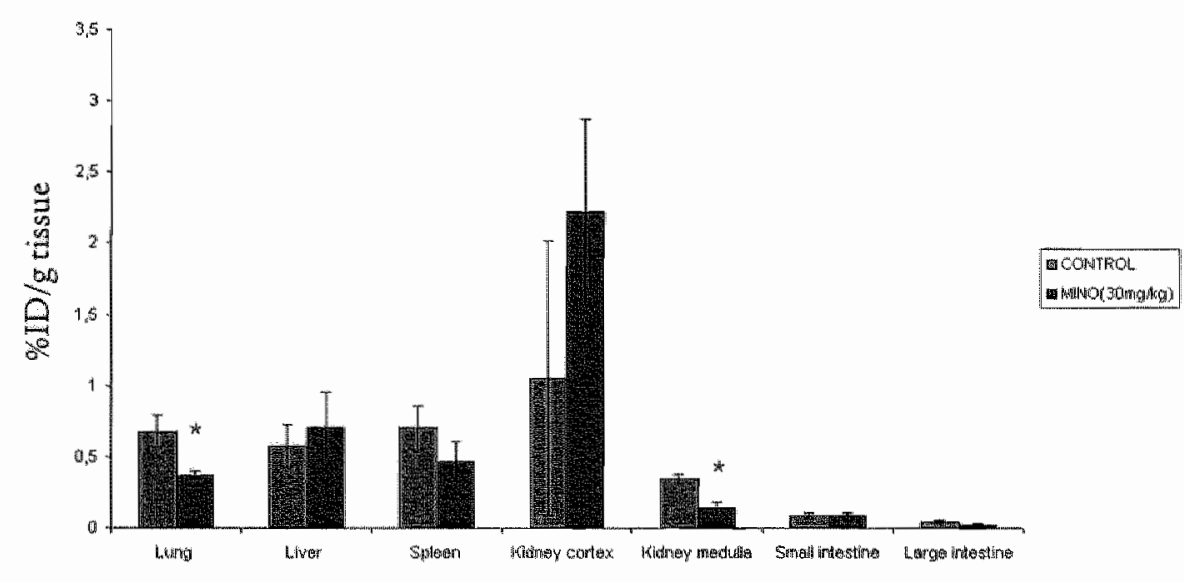

Figure 5.5: Uptake of ${ }^{99} \mathrm{Tc}$-Annexin A5-radioactivity (\%ID/g tissue) in N-Z White male rabbits after $40 \mathrm{~min}$ of ischemia and $3 \mathrm{~h}$ of reperfusion. Statistically significant differences $(p<0.05)$ between the organs of control rabbits and minocycline treated animals are indicated with an asterisk $\left({ }^{*}\right)$. 


\subsection{Discussion}

Cell death in ischemia and reperfasion

Over the past 15 years there has been a continuing and lively debate about the nature of cell death during myocardial injury ${ }^{100.164,165}$. Up until the nineties, it was believed that necrosis is the predominant cause of cell death during myocardial ischemia and subsequent reperfusion.

In 1994, however, Gottlieb and coworkers showed that rabbit cardionyocytes in vitro undergo an apoptosis-like form of cell death after exposure to I/R ${ }^{166}$. After these findings, more groups gathered evidence on apoptosis in myocardial injury, both in animal models ${ }^{165}$ as well as in post mortem cardiac tissue, collected from patients who died after a tecent myocardial infarction ${ }^{167,168}$.

\section{Measurement of cell deatb imbibition with annexin AS imaging}

During the past five years, we and others have gathered substantial evidence showing. that annexin A5 is feasible to detect cell death in the hearts of animals and patients undergoing ischemia and reperfusion ${ }^{62,66,68,100,101}$. It has been demonstrated that expression of PS is an important symptom, indicative for various forms of cell death ${ }^{74}$. Furthermore, it was revealed that caspase 3 is a driving force for PS-expression in cells during apoptosis ${ }^{80,81}$. Hence, imaging of PS-expression with the annexin A5 imaging protocol can be employed to measure the effect of cell death inhibitors on apoptosis and other possible forms of cell death in myocardial I/R injury.

As annexin $A 5$ binds specifically to PS, it is thus able to detect areas with high PSexpression using radiolabeling, fuorescent and immunohistological methods. The question remains whether annexin $\mathrm{A} 5$ is feasible to quantitatively detect the effect of cell death inhibition in animals and even in patients.

Dumont et al ${ }^{100}$ have alteady shown that the sodium hydrogen exchange inhibitor eniporide is able to cause a statistically significant decrease in the amount of apoptotic cells in the heart. In this study, we were able to measure PS-expression in mice and rabbit myocardial I/R quantitatively. By this means, it was possible to assess the pharmacological effect of minocycline on myocardial cell death after $I / R$ injury. It was shown that PS-expression could be inhibited significantly for at least $42 \%$ by 30 $\mathrm{mg} / \mathrm{kg}$ minocycline in both animal models.

Recently, Scarabelli et $a^{162}$ have described cell death inhibition by minocycline in vitro using an $I / R$ model with isolated cardiomyocytes as well as $I / R$ in perfused Langendorf-hearts, respectively. A beneficial effect of minocycline in these $I / R$ models was demonstrated.

The results from the studies described here provide new evidence for our hypothesis that annexin A5 imaging is able to quanrify cell death for comparison between treated and untreated animals. The decrease in PS-expresion caused by minocycline in these 
I/R models is likely to be due to a cell death inhibitory effect. Although the effect of minocycline was demonstrated in these two animal models at two different time points, the long-term beneficial effect of minocycline on I/R cell damage remains to be elucidated.

Scarabelli et al used the hearts of minocycline-pretreated rats and cell cultures to perform their experiments ${ }^{162}$. In our studies, however, we were able to mimic the clinical situation by administration of $30 \mathrm{mg} / \mathrm{kg}$ minocycline upon reperfusion.

The underlying mechanism of cell death inhibition by minocycline was demonstrated by Scarabelli et a $\left.\right|^{162}$. Interestingly, the amounts of both apoptotic and necrotic cells were found to be decreased, respectively. Moreover, they showed that the amount of TUNEL-positive cells as well as the caspase activation of caspase $-3,-7,-8$, and -9 was decreased upon minocycline treatment. Furthermore, an increase in the cytoplasmic ratio of XIAP to Smac/Diablo was observed concomitant with the inhibition of cytochtome $c$ release from mitochondria ${ }^{162}$. The latter suggests that minocycline is able to inhibit even non-caspase dependent programmed cell death forms ${ }^{74,169}$.

Minocycline is a relatively non-toxic compound and licensed for antibiotic indications. Although it was administrered at a high single dose, we expect that it will exert efficacy when it is given to patients during the first days after an acute myocardial infarction. Therefore, its use in patients suffering from myocardial infarction is an interesting target for novel research. Moreover, the protective effect of minocycline against cell death in inflammation was already shown in patients with rheumatoid arthritis over a long term treatment interval (1-2 years) ${ }^{170}$.

\section{Pbarmacokinetics/ Biodistribution of ${ }^{99}$ "To-HYNIC-arnexin A5 in rabbits}

To be sure that imaging is not affected by any treatment modality, it is important to evaluate biodistribution and pharmacokinetic data of treatment and control animals. This in order to ensure thar there are no artificial effects which may have affected the results obtained.

In this study, the clearance and biodistribution HYNIC-annexin A5 of minocycline treated rabbits was comparable to the same data obtained in control rabbits. Although uptake in two organs (lung, kidney medulla) was shown to be significantly different between the treatment group and the controls, we assume that these differences do not affect the results. It is still not clear what causes these biodistribution changes. Moreover, in another I/R study performed in Swiss mice, we showed that eniporide is able to cause a change in the biodistribution of BTAP-annexin ${ }^{171}$. These results triggered us to investigate the effect of other treatments regimens in I/R experiments on annexin innaging and biodistribution more carefully. 


\subsection{Conclusion}

By performing this study, we have shown that minocycline $(30 \mathrm{mg} / \mathrm{kg})$ is able to inhibit cell death during myocardial ischemia and reperfusion in both rabbits and mice. This effect was already postulated in vitro, but here we were able to quantify the effect of minocycline in $\mathrm{I} / \mathrm{R}$ using annexin $\mathrm{A} 5$ imaging procedures under mimicked clinical circumstances. Furthermore, we have observed that minocycline treatment only marginally affects ${ }^{99 m}$ Tc-HYNIC-annexin A5 biodistribution in rabbits. New studies are in progress to investigate the mechanisms of cell death inhibition by minocycline in vivo and to validate the observed cell. salvaging effect of minocycline in patients with myocardial infarction. 



General Discussion 


\subsection{Introduction}

Programmed cell death has a crucial role in physiological homeostasis as well as in pathology. Many drugs, such as cytostatics, affect the cell death process ${ }^{132}$. Thus, the detection and quantification of PCD in vivo can provide insight into the disease status and/ or treatment efficacy of a patient.

The externalisation of phosphatidyl serine (PS) at the cell membrane is one of the first events at the cell membrane of a dying cell and it also persists until the cell is destroyed ${ }^{59}$. Annexin A5, binds with high affinity to PS on dying cells. This quality seemed to be hardly affected by labeling with fluorescent or nuclear probes. Fortunately, it was possible to develop fuorescent and radiolabeled annexin A5, suitable as a molecular imaging tool. Fluorescent and radiolabeled annexin A5 were tested in animal models ${ }^{65,100}$.

Interestingly, the acrual development of annexin A5 as a cell death detecting agent occurred by chance. Having been discovered as a protein with anticoalguant properties, annexin $\mathrm{A} 5$ was already known as a PS-binding ligand ${ }^{14}$. This is because of the PS-expression on blood platelets, which is a target for annexin A5. It was revealed by Fadok and coworkers that apoptotic cells also have PS on their cell membranes ${ }^{59}$. 'This mechanism serves as an 'eat me-signal' to enhance phagocytosis ${ }^{114}$. Hereafrer it was demonstrated that annexin A5 binds to externalized PS on apoptotic cells with high affinity and specificity ${ }^{60,81}$.

Technetium labeled annexin A5 was performed for the first time in patient imaging at the Maastricht University Hospital. Using this methodology, imaging in patients with myocardial ischemia and subsequent reperfusion using technetium-labeled annexin $\mathrm{A} 5$ could be described ${ }^{66,68}$. It was also shown that the available technetium labeled probes were not suitable for imaging the abdomen ${ }^{55.87,105}$. This hampered for example the imaging of tumors in the colon, liver and kidney. Furthermore, the quality of annexin A5 preparations could not be evaluated accurately.

The purpose of this thesis was to gain insight and to develop methods to improve the annexin A5 nuclear imaging protocol. Because of the development and availability of new labeling methods in time, there was a need to use three different conjugation types of annexin A5 to ${ }^{99} \mathrm{Tc}$. We used HYNIC-annexin A5 for the research described in Chapters 2 and 5, research with the BTAP-annexin A5 conjugarion method was demonstrated in Chapters 3 and 4, whereas Imino-annexin A5 appeared only in Chapter 3.

In this thesis, the first part of the research described is about quality control of the biological functionaliry of annexin A5 preparations (Chapter 2). The second part of rhis thesis (Chapters 3 and 4) describes the imaging challenges and biodistribution pirfalls of the early technerium labelled annexin probes ${ }^{55,105}$. Chapter 5 shows how ${ }^{9} \%_{m}$ Tc-annexin A5 can be used as a treatment evaluation tool. Similarly, the first paragraphs in this discussion will be about the quality control of annexin A5. The 
second part of this discussion will be about biodistribution, imaging properties and their improvement options. The third part will be about the option to use annexin A5 imaging for treatment evaluation. It has become clear that annexin A5 based imaging is feasible for evaluation of (anti-) cell death treatment. But what is its clinical impact and future perspective? 


\subsection{Biofunctionality assessment of annexin A5 and its importance for imaging}

To assure the correctness of an imaging procedure, accurate quality control of the tracer is required. This is illustrated by the paper from Subbaryan et al. ${ }^{172}$. They demonstrated that annexin $A 5$ can easily be labeled with ${ }^{99}{ }^{\prime} \mathrm{Tc}$ using a hydrazide method in the presence of tricine, PDTA and stanous chloride. They achieved a high labeling efficiency using ITLC. However, a major pitfall in this study is the question whether the labeled annexin A5 is still bioactive. This is because the protein was heated to $90{ }^{\circ} \mathrm{C}$ for $10 \mathrm{~min}$. It has been demonstrated, however, that annexin $\mathrm{A5}$ denaturation already occurs at $60^{\circ} \mathrm{C}$ and $15 \mathrm{~min}$ of heating (Chapter 2$)^{20}$. So, it would be inaccurate to use ITLC alone in order to measure the biofunctionality of the radiopharmaceutical ${ }^{173}$.

The radiolabeling procedure of annexin A5 prior to injection into the patient is a step that should take a short time. This is because of the short half-life time of most radioisotopes, such as ${ }^{99 \mathrm{~m} T c}\left(\mathrm{t}_{1 / 2}= \pm 6 \mathrm{~h}\right),{ }^{18} \mathrm{~F}\left(\mathrm{t}_{1 / 2}= \pm 1.8 \mathrm{~h}\right)$ and ${ }^{68} \mathrm{Ga}\left(\mathrm{t}_{1 / 2}=68 \mathrm{~min}\right)$. The results of a physical analysis, such as TLC do not provide any information about the biological functionality, and consequently, the PCD imaging capacity of the radiolabeled annexin A5. Hence, there is a great need for a rapid and simple method to determine the biological activity of radiolabeled annexin $A 5$, which is prepared for clinical use. The development and initial validation of a rapid and simple method to measure the percentage of biologically active ${ }^{99 \mathrm{~m}} \mathrm{Tc}$-annexin $\mathrm{A} 5$ within the short time-window between preparation and injection of the radiopharmaceutical has been described (Chapter 2). It was demonstrated that this assay measures the biological activity of Annexin A5 that underlies its apoptotic cell. binding property. Furthermore, it has been shown that this assay is robust, repeatable and. reproducible. Its speed makes it suitable for inclusion in a clinical setting. The assay was named the ApoCorrect ${ }^{\text {an }}$ assay.

Another point that has been adressed using this biofunctionality assay, was the measurement of neutralizing antibodies. Repeated administration of ${ }^{99 \mathrm{~m}} \mathrm{Tc}$ annexin $\mathrm{A} 5$ to a patient may cause the generation of antibodies neutralizing the biological activity of annexin A5. As this had already occurred with some preparations containing erythropoeitin, regulatory authorities such as the FDA and EMEA sripulate availability of analytical procedures that measure neutralizing antibodies of compounds that are to be administered to patients parenterally. The ApoCorrect ${ }^{\text {tw }}$-assay was therefore adapted to determine the presence of antibodies neutralizing annexin A5. The assay was validated using a monoclonal antibody and polyclonal antibodlies against annexin A5 (see Chapter 1). Plasma samples of healthy, 99: Tc-annexin A5-naive volunteers were used as negative controls. It was demonstrated that the method was able to accurately detect the presence of neutralizing antibodies against annexin A5. So, annexin A5 biofunctionality assessment can be used in various ways for both quality control and patient autoantibody determination. 


\subsection{Biodistribution of radiolabeled annexin A5: how a pitfall remains a challenge}

Chapters 3 and 4 gave more insight into pharmacokinetics, imaging properties and biodistribution of Imino-annexin A5 and BTAP-annexin A5, respectively. The main. disadvantage of Imino-annexin A5 was the overall high uptake of the tracer in liver, kidney, spleen and gut combined with a long biological half life. The predominant disadvantage of BTAP-annexin A5 was its high and fast radioactivity uptake in the gut. The latter combined with high radioactivity uptake in the same organs as for Imino-annexin A5 made the compound suitable only for imaging in the thorax, head and neck region, as well as the extremities (Chapter 3) 55.105 . Furthermore, it was demonstrated that the biodistribution of radiolabelled annexin A5 is markedly influenced by the conjugation method used (Chapter 3$)^{55}$.

The poor biodistribution properties of both Imino-annexin A5 and BTAP-annexin A5 triggered us to try to find a way to improve biodistribution. By then, it appeared. that there were rwo ways to improve annexin A5"s biodistriburion: eirher by changing the protein or by changing the labeling method.

A change in the protein has been attempted. Unfortunately, it was not possible to include the results obtained in this thesis. An in vitro study showed the structureactivity relationship of inactivated domain I $\mathrm{Ca}^{2+}$ type II binding sites of Annexin A5 and its inhibitory activity on cellular processes ${ }^{32}$. No data were available yet on the biodistribution of these annexin A5 derivatives. Therefore, the purpose of the study was to investigate the biodistribution of BTAP-annexin A5 derived proteins using annexin A5-mutants in which one or more of the the four different type II high affinity binding sites are disabled. We were however not able to perform reproducible labeling of mutants using the BTAP method. Moreover, most mutants showed even higher uptake in the liver and spleen of Swiss mice than wild type BTAP annexin A5 and were therefore not suitable candidates for improved imaging with annexin A5 (H.H. Boersma, E.A. Dumont, et al, unpublished observations). Although we still intended to perform new experiments using HYNIC labelled mutants of annexin A5, the publication of Tait et al about the biodistribution of annexin A5 mutants with disabled $\mathrm{Ca}^{2+}$ type II binding sites prevented further studies ${ }^{174}$. "They showed that the blood clearance of annexin A5 was not affected by its overall net charge and affinity of binding to PS. On the basis of their results they postulate that the clearance by the kidneys is a PS-independent process, whereas uptake in normal liver and spleen decreased with the PS-affinity of the changed annexin A5 moleculle. The same pattern was obserwed in animals treated with cycloheximide to induce apoptosis. Control experiments with charge mutants showed that the effects seen with the affinity mutants were not due to the concomitant change in molecular charge that occurs in these mutants. They conclude that all four domains of arnexin. A5 are required for optimal uptake in apoptotic tissues. Annexin A5-derivatives with 
only one or two PS-binding sites are unlikely to be suitable for imaging of cell death in vivo. They suggest that uptake in normal liver and spleen is specific whereas renal uptake is nonspecific ${ }^{174,175}$.

At least, one change in the labeling method turned out to be more fruitful. The advent of HYNIC-annexin A5 for use in patients as well as in animals gave us an annexin A5 radiotracer with improved biodistribution ${ }^{65,90,121}$. This evolution shifted the focus of this thesis to other topics such as treatment evaluation and quality control of the radiopharmaceutical.

The currently used HYNIC-annexin A5 radiopharmaceutical has a good biodistribution profile and is suitable for regular clinical use, but still some needs are unmet. HYNIC-annexin A5, even more than Imino-annexin A5 and BTAP. annexin $\mathrm{A} 5$, shows very high uptake in the kidney, thus hampering imaging of cell death within this organ.

${ }^{123}$ I-annexin A5 and the recently developed DTPA/DOTA-annexin A5 are other examples that show less uptake in at least the kidney. The rather complex labeling method used for ${ }^{123} \mathrm{I}$-annexin A5 is expected to hamper further clinical development ${ }^{96}$. However, for DTPA-annexin A5 labeled with ${ }^{111} \mathrm{In}$ and ${ }^{68} \mathrm{G}$ a promising results were obtained in animal studies with SPECT $\left({ }^{111} \mathrm{I} n\right){ }^{176}$ and PET $\left({ }^{68} \mathrm{Ga}\right)$ imaging (S.L. Woleers et al, unpublished observations). The paper by Sarai et al demonstrates that the kidney uptake $3 \mathrm{~h}$ after injection of ${ }^{111} \mathrm{In}$-DTPA-annexin $\mathrm{A} 5$ in rabbits is about half of the \%ID/g tissue measured for HYNIC-annexin A5 at the same timepoint ${ }^{176}$. Using ${ }^{68} \mathrm{Ga}$-DTPA-annexin A5 we did not observe evident kidney uptake after administration of the tracer in a rat after an I/R experiment (S.L. Wolters et al, unpublished observations).

Hence it is clear that the development of conjugates with a better biodistribution profile should be continued. The research group of Tair, who used endogenous chelation sites for ${ }^{9 \%} \mathrm{~m} T \mathrm{C}$-labeling of annexin A5, shows promise as well in this respect. It leads to better target/ background ratios and less uptake in the liver, kidney and spleen ${ }^{174}$. They showed that the biodistribution of annexin A5 is influenced by the labeling method. For example, the annexin A5 mutant 128 with endogenous ${ }^{99 \mathrm{~m}} \mathrm{TC}$ chelation sites is taken up by the apoptotic cell to a similar or even better extent than HY"NIC-annexin A5. Furthermore they demonstrated that mutant 128 has a much lower renal uptake at $60 \mathrm{~min}$ after injection. The latter was suggested to be due to a more rapid urinary excretion of radioactivity in the case of annexin A5-mutant $128^{174,175}$. It is evident that the progress, described above, in the development of annexin A5 imaging tracers will sustain its use and clinical application in the future. 


\subsection{From imaging feasibility to treatment evaluation, and beyond.......}

Biodistribution bothered us in the first years of radiolabelled annexin A5 utilisation. Because of the high uptake in the liver, kidney and spleen, a long time interval was required between administration of the tracer and, for example Acute Myocardial Infarction (AMI) imaging (15-20 h). Still, little is known about the necessary time frame between administration of radiolabelled annexin $A 5$ and subsequent imaging. It is assumed that this is caused by a coincidence of biodistribution features of the used radiopharmaceutical with the targeted cell death process. For instance, the obtained imaging results with BTAP-annexin A5 show that it is able to vizualize a breast tumor $4 \mathrm{~h}$ after adminustration of BTAPannexin A5. This is in marked contrast with experiences in AMI imaging using HYNIC annexin $A 5^{55}$. Using HYNIC-annexin A5, however, AMI can be imaged best $4.6 \mathrm{~h}$ after administration of the compound (L. Hofstra, personal communication) $)^{175}$.

Another very interesting question remains about what is actually assessed by PSexpression imaging. Is this cell death or more? And what is cell death? New and renewed insights on this topic are in a fast developmental mode at this moment. Recently, an intriguing review appeared on the natures of cell death ${ }^{169}$. Furthermore, based on the former review, a related review on the disguises of cell death and the role of PS-expression in the appearance of several cell death forms will be published soin ${ }^{17}$.

To be able to perform therapy evaluation using annexin A5, more information must be gathered in order to validate the use of annexin A5 for (anti-)apoptotic treatment of disease in humans. The later would be of great interest for the prediction of treatment outcome in patients. For instance, the use of annexin $\mathrm{A} 5$ to deremine the efficacy of chemotherapy or radiation therapy in cancer in order to be able to predict patient survival would be of great value.

Another interesting approach is the evaluation of cell death inhibition, as has been demonstrated in Chapter 5 for minocycline treated myocardial $\mathbb{I} / \mathbb{R}$ and subsequent reperfusion in mice and rabbits. It is evident that imaging with annexin. $\mathrm{A} 5$ probes can be developed as an important tool to evaluate drugs regarding their cell death inhibitory or PCD inducing properties. Large clinical trials should be conducted to prove the feasibility of these methods in patients.

Simultaneous measturements of cell proliferation, cell death and cellular oxygen consumption using radiopharmaceuticals labeled with different radionuclides, is the ultimate goal of radiotherapists in particular, in order to individualize patient treatment. Nuclear Medicine could play an important role in this fascinating $\operatorname{approach}^{175}$. 


\subsection{Future Perspectives}

Based on the research described in this thesis, new developments can be made in the near future. Apart from the issues already mentioned in this discussion, the following items are of interest:

- A method with high potential in patient management is e.g. the determination of tumor malignancy in particular tumor types. There is some evidence that highly malignant tumors show high PS-expression. More clinical evidence is needed to warrant the feasibility of this method ${ }^{175}$.

- The development of annexin A5 containing pharmaceuticals for the targeting of drug treatment in diseases like cancer would enhance the ability to treat diseases more specifically. Targeted therapy presumably increases treatment efficacy and similarly decreases the probability of adverse reactions in patients. The property of annexin A5 to internalize within a cell is certainly of interest regarding treatment options. The ability of the annexin A5 molecule to serve as a carrier for therapeutics is the subject of further research ${ }^{175}$. 



$$
7
$$



At last... 


\subsection{References}

1. Gerke V, Moss SE. Annexins: from structure to function. Pbysiol Rev. 2002;82:331-71.

2. Bohn H, Kraus W. [Isolation and characterization of a new placenta specific protein (PP10) (author's rransl)]. Arch Gynecol. 1979;227:125-34.

3. Reutelingsperger CP, Hornstra $G$, Hemker HC. Isolation and partial purification of a novel anticoagulant from arteries of human umbilical cord. Eur J Biochem. 1985;151:625-9.

4. Crumpton MJ. Dedman JR. Protein terminology tangle. Nature. 1990;345:212.

5. Kobayashi R, Tashima Y. Identification and partial separation of three distinct 32 $\mathrm{kDa}$ calcium/phospholipidregulated proteins from bovine spleen. Biochem Biopbys Res Commun. 1989:162:15-23.

6. Reurelingsperger $C$, van Heerde W, Hauptmann $R$, Maassen $C$, wan Gool RG, de Leeuw $P$, Tiebosch A. Differential tissue expression of Annexin VIII in human. FEBS Lett. 1994;349:120 4

7. Kaetzel MA, Hazarika P, Dedman JR. Differential tissue expression of three 35-kDa annexin calcium-dependent phospholipid-binding proteins. J Biol Chem. 1989;264:14463-70.

8. Maurer-Fogy 1, Reutelingsperger CP, Pieters J, Bodo G, Stratowa C, Hauptmann R. Cloning and expression of CDNA for human vascular anticoagulant, a $\mathrm{Ca} 2+$-dependent phospholipid-binding protein. Eur J Biochem. 1988;174:585-92.

9. Kaplan R, Jaye M, Burgess WH, Schlaepfer DD, Haigler HT. Cloning and expression of cDNA for human endonexin II, a Ca2+ and phospholipid binding protein. J Biol Chem. $1988 ; 263: 8037-43$.

10. Iwasaki A, Suda M, Nakao H, Nagoya T, Saino Y, Arai K, Mizoguchi T, Sato F, Yoshizaki $H$, Hirata $M$, et al. Structure and expression of $c D N A$ for an inhibitor of blood coagulation isolated from human placenta: a new lipocortin-like protein. J Biochem (Tokyo). 1987;102:1261-73.

11. Cookson BT, Engelhardr S. Smith C, Bamford HA, Prochazka M, Tair JF. Organizarion of the human annexin V (ANX5) gene. Genomics. 1994;20:463-7.

12. Hauptmann R, Reutelingsperger, CPM. Molecular biology and biochemistry of annexins $V$ and VIII. In: Moss S. (ed.) The Annexims Chapel-Hill. 1993:139-152.

13. Geisow MJ, Fritsche U, Hexham JM, Dash B, Johnson T. A consensus aminomacid sequence repear in Torpedo and mammalian $\mathrm{Ca} 2+$-dependent membrane-binding proteins. Nature. 1986;320:636-8.

14. wan Heerde WL, de Groot PG, Reurelingsperger CP. The complexity of the phospholipid binding protein Annexin V. Thromb Hoemost. 1995;73:172-9.

15. Seaton BA. In: (Seaton, B.A., ed) Annexins: Molecular structure to cellular function, R.G. Landes Company, Austin, TX. 1996:15-29.

16. Pearson DA, Lister J, MaBride WJ, Wilson DM, Martel LJ, Civirello ER, Dean RT. Thrombus inaging using technetium-99m-labeled high-potency GPIIb/IIIa receptor antagonists. Chemistry and initial biological studies. J Med Chem. 1996;39:1372-82.

17. Yang DJ, Azhdarinia A, Wu P, Yu DF, Tansey W, Kalini SK, Kim EE, Podoloff DA. In vivo and in vitco measurement of apoptosis in breast cancer cells using $99 \mathrm{mTc}$.EC-annexin V. Cancer Biather Radiopbarm. 2001;16:73-83.

18. Lienanm $S_{y}$ Lewit-Bentley $A$. Annexins: a novel family of calcitm- and membrane-binding proteins in seard of a function. Structure. 1995;3:233-7.

19. Kohlet G, Hering U, Zschomig O, Annold K. Annexin V interaction with phosphatidylserinecontaining vesicles at low and neutral $\mathrm{pH}$. Biochemistry. 1997;36:8189-94.

20. Boersma HH, Srolk LM, Kenis H, Deckers NM, Vanderheyden JL, Hofstra L, Heidendal GA, Reurelingsperger $\mathrm{CP}$. The A poCorrect assay: a novel, rapid method to determine 
the biological functionaliry of radiolabeled and fuorescent Annexin A5. Anal Biochem. 2004:327:126-34.

21. Brisson $A$, Mosser $G$, Huber $R$. Structure of soluble and menbrane-bound human annexin V.J Mol Biol. 1991;220;199-203.

22. Lewir-Bentley A, Doublie S, Fourme R, Bodo G. Crystallization and preliminary X-ray studies of human vascular anticoagulant protein.J Mol Biol. 1989;210:875-6.

23. Concha NO, Head JF, Kaetzel MA, Dedman JR, Seaton BA. Rat annexin V crystal structure: $\mathrm{Ca}(2+)$-induced conformational changes. Sciente. 1993;261:1321-4.

24. Burger A, Voges D, Demange P, Perez CR, Huber R, Berendes R. Structural and elecrophysiological analysis of annexin $V$ mutants. Mutagenesis of human annexin $V$, an in witro voltage-gated calcium channel, provides information about the structural features of the ion partway, the voltage sensor and the ion selectivity filter. I Mol Biol. 1994;237:479-99.

25. Weng X, Luecke H, Song IS, Kang DS, Kim SH, Huber R. Crystal structure of human annexin I at 2.5 A resolution. Protein Sci. 1993:2:448-58.

26. Andree HA, Reutelingsperger CP, Hauptmann R, Hemker HC, Hermens WT, Willems GM. Binding of vascular anticongulant alpha (VAC alpha) to planar phosphollipid bilayers. J Biol Cherm. 1990;265:4923-8.

27. Swairjo MA, Concha NO, Kaetzel MA, Dedman JR, Seaton BA. Ca(2+)-bridging mechanism and phospholipid head group recognition in the membrane-binding protein annexin V. Nat Stract Biol. 1995;2:968-74.

28. Oling F, Bergsma-Schutter W, Brisson A. Trimers, dimers of trimers, and trimers of trimers are common building blocks of annexin a 5 two-dimensional crystals. I Struct Biol. $2001 ; 133: 55-63$.

29. Isas JM, Cartailler JP; Sokolov Y, Patel DR, Langen R, Luecke H, Hall JE, Haigler HT. Annexins $\mathrm{V}$ and $\mathrm{XII}$ insert into bilayers at mildly acidic $\mathrm{pH}$ and form ion channels. Biochemistry. 2000:39:3015-22,

30. Stuart MC, Reutelingsperger CP, Frederik. PM. Binding of annexin V to bilayers with various phospholipid compositions using glass beads in a flow cytometer. Cytometry. $1998 ; 33: 414-9$.

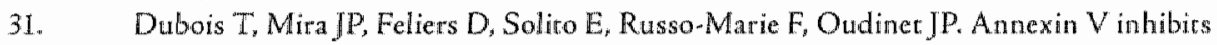
protein kinase $C$ activity via a mechanism of phospholipid sequestration. Biochem J. $1998 ; 330(\operatorname{Pr} 3): 1277-82$

32. Mira JP, Dubois T, Oudinet JP, Lukowski S, Russo-Marie F, Geny B. Inhibition of cytosolic phospholipase A2 by annexin $V$ in differentiated permeabilized HL-60 cells. Evidence of crucial importance of domain I type II Ca $2+$-binding site in the mechanism of inhibition.J Bial Chem. 1997;272:10474-82.

33. Montaville P, Neumann JM, Russo-Marie F, Ochsenbein F, Sanson A. A new consensus sequence for phosphatidylserine recognirion by annexins. J Biol Chem. 2002;277:24684-93.

34. Jin M, Snith C, Hsieh HY, Gibson DF, Tait JF. Essential Role of B-helix Calctum Binding Sites in Annexin V-Membrane Binding.J Biol Cbem. 2004;279:40351.7.

35. Fabisiak JP, Tyurina YY, Tyurin VA, Lazo JS, Kagan VE. Random versus selective membrane phospholipid oxidation in apoptosis: role of phosphatidylserine. Biochemistry. $1998 ; 37: 13781-90$.

36. Kagan VE, Borisenko GG, Serinkan BF, Tyurina YY, Tyurin VA, Jang ], Liu SX, Shvedova AA, Fabisiak JP, Uthaisang W, Fadeel B. Appetizing rancidity of apoptotic cells for macrophages: oxidation, externalization, and recognition of phosphatidylserine. Am J Physiol Liang Cell Mol Physiol. 2003;285:L1-17.

37. Reurelingsperger CP, Kop JM, Hornstra $G$, Hemker HC. Purification and characterization of a novel protein from bovine aorta that inhibits coagulation. Inhibition of the phospholipid-dependent factor-Xa-catalyzed prothrombin activation, through 
a high-affinity binding of the anticoagulant to the phospholipids. Eur I Biochem. 1988:173:171.8.

38. Raynal P, Pollard HB. Annexins: the problem of assessing the biological role for a gene family of multifunctional calcium- and phospholipid-binding proteins. Biochim Biopbys Acta. 1994;1197:63-93.

39. Dawidson FF, Dennis EA, Powell M, Glenney JR, Jr. Inhibition of phospholipase A2 by "lipocortins" and calpactins. An effect of binding to substrate phospholipids. J Biol Chem. 1987:262:1698-705.

40. Comera $\mathrm{C}$, Rothlihut $\mathrm{B}$, Russo-Marie F. Identification and characterization of phospholipase A2 inhibitory proteins in human mononuclear cells. Eur J Biochem. 1990:188:139-46.

41. Homaidan FR $C_{*} I_{*}$ Haidar HA, El-Sabban ME. Protein regulators of eicosanoid synthesis; role in inflammation. Curr Protein Pept Sir. 2002;3:467.484.

42. Aarsman Al, Mymbeek G, wanden Bosch $H$, Roththut B, Prieur B, Comera C, Jordan L, Russo-Marie F. Lipocortin inhibition of extracellular and intracellular phospholipases A2 is substrate concentration dependent. FEBS Lett. 1987;219:176-80.

43. Musashi M, Ora, S, Shiroshita N. The role of protein kinase C isoforms in cell proliferation and apoptosis. Int J Hematol. 2000;72:12-19.

44. Russo-Marie E. Annexin V and phospholipid metabolism. Clim Chem Lab Med. $1999,37: 287-91$.

45. Hynes R, Zhao Q. The evolution of cell adhesion. J Cell Biol. 2000;150: F189-F196.

46. Andersen $\mathrm{MH}_{3}$, Berglund L, Petersen TE, Rasmussen JT. Annexin-V binds to the intracellular patt of the beta(5) integrin receptor subunit. Biocbem Biopbys Res Commun. $2002 ; 292: 550-7$.

47. Hoffmann P, deCathelineau AM, Ogden CA, Leverrier Y, Bratton DL, Daleke DL, Ridley A], Fadok VA, Henson PM. Phosphatidylserine (PS) induces PS receptor-mediated macropinocytosis and promotes clearance of apoptotic cells. I Cell Biol. 2001;155:649-659.

48. Kenis $H$, van Genderen $H$, Bennaghmouch A, Rinia HA, Frederik P, Narula J, Hofsera L, Reurelingsperger CP. Cell Surface-expressed Phosphatidylserine and Annexin A5 Open a Novel Portal of Cell Entry. J Biol Chem. 2004;279:52623-9.

49. van den Eijnde SM, van den Hoff MJ, Reutelingsperger CP, van Heerde WL, Henfling ME, Vermej-Keers C, Schurte B, Borgers M, Ramaekers FC. Transient expression of phosphatidylserine at cell-cell contact areas is required for nyotube formation. I Cell Sci. 2001;114:3631-42.

50. Kubista H, Hawkins TE, Patel DR, Haigler HT, Moss SE. Annexin 5 mediates a peroxide-induced $\mathrm{Ca}(2+)$ influx in B cells, Curr Biol, 1999;9:1403-6.

51. Kirsch T, Nah HD, Demurh DR, Harrison G, Golub EE, Adams SL, Pacifici M. Annexin $V$ mediaced calcium fux across membranes is dependent on the lipid composition: implications for cat tilage mineralization. Biochemistry. 1997;36:3359-67.

52. Arispe N, Rojas E, Genge BR, Wu LN, Wuthier RE. Similarity in calcium channel activity of annexin $V$ and matrix vesicles in planar lipid bilayers. Bioplyys.J. 1996;71:1764-75.

53. Hachida M, Lti H, Kaneko N, Horikawa Y, Ohkado A, Gu H, Zhang XL, Hoshi H, Nonoyama M, Nakanishi T, Koyanagi H. Protective effecr of JTV519 (K201), a new 1,4-benzothiazepine derivative, on prolonged myocardial preservation. Transplant Proc. 1999:31:996-1000.

54. Brachvogel B, Dikschas J, Moch H, Welzel H, von der Mark K., Hofmann C, Poschl E. Annexin A5 is not essential for skeleral development. Mol Cell Biol. 2003;23:2907-13.

55. Boersma HH, Liem IH, Kemerink G], Thimister PW, Hofstra Ls Stolk LM, van Heerde WL, Pakbiers MT, Janssen D, Beysens AJ, Reutelingsperger CP, Heidendal GA. Comparison between human pharmacokinerics and inaging properties of two conjugation methods for 99mTc-annexin A5. BrJ Radiol. 2003;76:553-60. 
56. Kaneko N, Marsuda R, Hosoda S, Kajita T, Ohta Y. Measurement of plasma annexin $V$ by ELISA in the early detection of acute myocardial infarction. Clin Chim Acta. 1996;251:65-80.

57. Song G, Campos B, Wagoner LE, Dedman JR, Walsh RA. Altered cardiac annexin mRNA and protein levels in the left ventricle of patients with end-stage heart failure. JMol Cell Cardiol. 1998;30:443-51.

58. Benevolensky D. Belikova X, Mohammadzadeh R. Trouve P Marorte F, Russo-Marie F, Samuel JL, Charlemagne D. Expression and localization of the annexins II, V, and. VI in myocardium from patients with end-stage heart failure. Lab Iswest. 2000;80:123-33.

59. Fadok V, Voelker DR, Campbell PA, Cohen JJ, Bratton DL, Henson PM. Exposure of phosphatidylserine on the surface of apoptotic lymphocytes triggers specific recognition and removall by macrophages. J Immunol. 1992;148:2207-2216.

60. Koopman G, Reutelingsperger CP, Kuijten GA, Keehnen RM, Pals ST, wan Oers MH. Annexin $V$ for flow cytometric detection of phosphatidylserine expression on $B$ cells. undergoing apoptosis. Blood. 1994;84:1415-20.

61. Kenis H, van Genderen H, Bennaghmouch A, Rinia HA, Frederik P, Narula J, Hofstra L, Reutelingsperger CP. Cell Surface-expressed Phosphatidylserine and Annexin A5 Open a Novel Portal of Cell Entry.J Biol Chem. 2004;279:52623-9.

62. Dumont EA, Reutellingsperger CP, Smits JF, Daemen MJ, Doevendans PA, Wellens H], Hofstra L. Real-time imaging of apoptotic cell-membrane changes at the single-cell level in the beating murine heart. Nat Med, 2001;7:1352-5.

63. Glaser M, Collingridge DR, Aboagye EO, Bouchier-Hayes L, Hutchinson OC, Martin S], Price P, Brady F, Luthra SK. Iodine-124 labelled annexin-V as a potential radiotracer to study apoptosis using positron emission tomography. Appl Radiat Isot, 2003;58:55-62.

64. Murakami Y, Takamatsu $H$, Taki J, Tatsumi M, Noda A, Ichise R, Tait JF, Nishimura S. 18 F-labelled annexin V; a PET tracer for apoptosis imaging. Eur J Nud Med Mol Inaging. 2004;31:469-74.

65. Blankenberg FG, Katsikis PD, Tait JF, Davis RE, Naumovski L, Ohtsuki K, Kopiwoda S, Abrams MI, Darkes M, Robbins RC, Maecker HT, Strauss HW. In vivo detection and. imaging of phosphatidylserine expression during programmed cell death. Proc Natl Acad Sci U S A. 1998:95:6349-54.

66. Hofstra L, Liem IH, Dumont EA, Boersma HH, van Heerde WL, Doevendans PA, De Muinck E, Wellens HJ, Kemerink GJ, Reutelingsperger CP, Heidendal GA. Visualistation of cell death in vivo in patients with acute myocardial infarction. Lawcet. 2000;356:209-12.

67. Hofstra L, Dumont EA Thimister PW, Heidendal GA, DeBruine AP, Elenbaas TW, Boersma $\mathrm{HH}$, wan Heerde WL, Reutelingsperger $\mathrm{CP}$. In vivo detection of apoptosis in an intracardiac rumor. Jama. 2001;285:1841-2.

68. Thimister PW, Hofstra L, Liem IH, Boersma HH, Kemerink G, Reutelingsperger $\mathrm{CP}$, Heidendal GA. In vivo detection of cell death in the atea at risk in acute myocardial infarcuion. J Nucl Med. 2003;44:391-6.

69. Haas RL, de Jong D, Valdes Olmos RA, Hoefnagel CA, van den Heuvell, Zerp SF, Barrelink $\mathrm{H}$, Verheij $\mathrm{M}$. In vivo imaging of radiation-induced apoptosis in follicular lymphoma patients. Int J Radiat Oncol Biol Phys. 2004,59:782-7.

70. van de Wiele C, Lahorte C, Vermeersch H, Loose D, Mervillie K, Steinmetz ND, Vanderheyden JL, Cuvelier CA, Slegers G, Dierck RA. Quantitative tumor apoptosis imaging using technetium-99m-HYNIC annexin V single photon emission computed tomography.J Clin Oncol. 2003;21:3483-7.

71. Kietselaer B, Thimister PWL, Reutelingsperger CPM, Heidendal GAK, Dunont EAWJ,

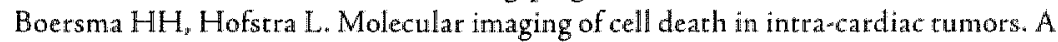
new approach to differential diagnosis in cardiac tumors. Netherlands Heart Jow mal. 2002;10:312-316. 
72. Narula Ji Acio ER, Narula N, Samuels LE, Fyfe B, Wood D, Fitzparrick JM, Raghumach PN, Tomaszewski JE, Kelly C, Steinmetz N, Green A, Tait JF, Leppo J, Blankenberg FG, Jain D, Strauss HW. Annexin- $V$ imaging for noninvasive detection of cardiac allograft rejection. Nat Med. 2001;7:1347-52.

73. Kietselaer BL, Hofstra L, Dumont EA, Reutelingsperger CP, Heidendal GA. The role of labeled Annexin A5 in imaging of programmed cell death. From animal to clinical imaging: Q N Nad Med. 2003;47:349-61.

74. Leist $M$, Jaattela $M$. Four dearhs and a funeral: from caspases to alternative mechanisms. Nat Rev Mol Cell Biol. 2001;2:589-598.

75. Blankenberg FG, Strauss HW. Will imaging of apoptosis play a role in clinical care? A tale of mice and men. A poptosis. 2001:6:11723.

76. Wyllie A. Glucocorticoid-induced thymocyte apoptosis is associated with endogenous endonuclease activation. Nature. 1980;284:555-556.

77. Wyllie AH, Kerr JF, Curtie AR. Cell deatt: the significance of apoptosis. Int Rev Cytol. $1980 ; 68: 251-306$.

78. Wyllie A, Kerr JE, Currie AR. Apoptosis: a basic biological phenomenon with wideranging inulications in tissue kinetics. Br J Cancer. 1972;26:237-257.

79. Hengartner M. The biochemistry of apoptosis. Nature. 2000;407:770.776.

80. Nusbaum P, Laine C, Seveau S, Lesavre P, Halbwachs-Mecarelli L. Early membrane events in polymorphonuclear cell (PMN) apoptosis: membrane blebbing and vesicle release, CD43 and CD16 down-regulation and phosphatidylserine externalization. Biochem Soc Trans. 2004;32:477-9.

81. Martin SJ, Reutelingsperger CP, McGahon AJ, Rader JA, van Schie RC, LaFace DM, Green DR. Eatly redistribution of plasma nembrane phosphatidylserine is a general. feature of apoptosis regardless of the initiaring stimulus: in hibition by overexpression of Bcl-2 and Abl.J Exp Med. 1995;182:1545-56.

82. Reutelingsperger CP. Annexins: key regularors of haemostasis, thrombosis, and apoptosis. Thromb Haemost. 2001; 86:413-9.

83. Dillon SR, Mancini M, Rosen A, Schlissel MS. Annexin V binds to viable B cells and colocalizes with a marker of lipid rafts upon B cell recepror activation. J Tmmunol. 2000;164:1322-32.

84. Verbruggen A. Bifunctional chelators for technetium-99m. In: Mother Sy (ed). Current directions in radiophamacentical research and development. Kluwer Academic Publishers. Dordrecht, Boston, London, 1996:31-46.

85. Lahorte CM, Vanderheyden JL, Steinmetz $\mathbb{N}$, Van de Wiele C, Dierckx RA, Slegers G. Apoptosis detecting radioligands: current state of the att and future perspectives. Eur J Nad Med Mol Imaging. 2004:31:887-919.

86. Goedemans W, Panek KJ, Ensing GJ, de Jong MTM. A new simple merhod for labeling of proceins with "9yurTc by derivatization wich 1-imino-4-mercaptoburyl groups. In. Nicolini $M$, Bandolini $G$, Mazzi $U$, (eds.) Technetium and rbenium in chemistry and nuclear medicine 3: Verona, Cortina International and New York: Raven Press. 1990:595-604.

87. Kemeeink GJ, Liem IH, Hofstra L, Boersma HH, Buijs WC, Reutelingsperger CP, Heidendal GA. Patient dosimetry of intravenously administered $99 \mathrm{~m} \mathrm{Tc}$ annexin $\mathrm{V}$.J Nud Med. 2001;42:382-7.

88. Kasina S, Rao TN, Srinivasan A, Sanderson JA, Fitzner IN, Reno JM, Beaumier PL, Fritzberg AR. Development and biologic evaluation of a kir for preformed chelate technetium-99m radiolabeling of an antibody Fab fragment using a diamide dimercaptide thelating agent. I Nud Med. 1991:32:1445-51.

89. Verbeke K, Kieffer D, Vanderheyten JL, Reurelingsperger C, Steinmetz N, Green A, Verbruggen A. Optimization of the prepararion of 99 mic-labeled Hynic-derivatized Annexin $V$ for human use. Nud Med Brol. 2003;30:771-8. 
90. Kemerink GJ, Liu X, Kieffer D, Ceyssens S, Mortelmans L, Verbruggen AM, Steinmetz ND, Vanderheyden JL, Green AM, Verbeke K. Safety, biodistribution, and dosimetry of 99 m Tc-HYNIC-annexin $V$, a novel human recombinant annexin $V$ for human application. J Nud Med. 2003;44:947-52.

91. TaitJF, Brown DS, Gibson DF, Blankenberg FG, Strauss HW. Development and characterization of annexin $V$ mutants wich endogenous chelation sites for $(99 \mathrm{~m}) \mathrm{Tc}$. Bioconing Cbem. 2000;11:918-25.

92. Tait JF, Smith C, Gibson DF. Development of annexin V mutants suitable for labeling with Tc(i)-carbonyl complex. Bioconitug Chem. 2002;13:1119-23.

93. Tair JF, Cerqueirat MD, Dewhurst TA, Fujikawa K, Ritchie JL, Stratton JR. Evaluation of annexin $\mathrm{V}$ as a platelet-directed thrombus targeting agent. Thromb Res. 1994;75:491-501.

94. Russell J, O'Donoghue JA, Finn R, Koziorowski J, Ruan S, Humm JL, Ling CC. lodination of annexin $V$ for imaging apoptosis. I Nud Med. 2002;43:671-7.

95. Lahorte C. Slegers G. Philippe J, Van de Wiele C, Dierckx RA. Synthesis and in vitro evaluation of 123I-labelled human recombinant annexin V. Biomol Eng. 2001;17:51-3.

96. Lahorte $C M$, van de Wiele $C$, Bacher $K$, wan den Bossche B, Thierens $H_{\text {, van }}$ Belle $\mathrm{S}$, Slegers $G$, Dierckx RA. Biodistribution and dosimetry study of 1231 -rh-annexin $V$ in mice and humans. Nucl Med Commun. 2003;24:871-80.

97. Zijlstra S, Gunawan I, Burchert W. Synthesis and evaluation of a 18F-labelled recombinant annexin- $V$ derivative, for identification and quantification of apoptotic cells with PET. Appl Radiat Isot. 2003;58:201-7.

98. Wen X, Wu QP, Ke S, Wallace S, Charnsangavej C, Huarng P, Liang D, Chow D, Li C. Improved radiolabeling of PEGylated protein. PEGylated annexin $V$ for noninvasive. imaging of tumor apoptosis. Cancer Brother Radiopharm, 2003;18:819-27.

99. van den Eijnde SM, Luijsterburg AJ, Boshart L, De Zeeuw $\mathrm{CI}_{n}$ van Dierendonck $\mathrm{JH}$, Reutelingsperger CP, Vermeij-Keers $\mathrm{C}$. In situ detection of apoptosis during embeyogenesis with annexin $V$ : from whole mount to ultrastructure. Cytometry. $1997 ; 29: 313-20$.

100. Dumont EA, Hofstra L, van Heerde WL, van den Eijnde S, Doevendans PA, DeMuinck E, Daemen MA, Smits JF, Frederik P, Wellens HJ, Daemen MJ, Reutelingsperger CP. Cardiomyocyte death induced by myocardial ischemia and reperfusion: measurement with recombinant human annexin- $V$ in a mouse model. Circulation. 2000;102:1564-8"

101. Taki J, Higuchi T, Kawashima A, Tait JF, Kinuyad S, Muramori A, Matsunaril, Nakajina K, Tonami N, Strauss HW, Detection of cardiomyocyte death in a model of ischenia and reperfusion using 99mTc-labeled annexin V.J Nicl Med. 2004;45:1536-41.

102. Mochizuki T, Kuge Y, Zhao S, Tsukamoto E, Hosokawa M, Strauss HW, Blankenberg FG, Tait JF, Tamaki N. Detection of apoptotic tumor response in vivo after a single dose of chemotherapy with 99 niTc-annexin V.J Nud Med. 2003;44:92-7.

103. Kuge Y, Sato M, Zhao S, Takei T, Nakada K, Seki KI, Strauss HW, Blankenberg FG, Tait JF, Tamaki N. Feasibility of $99 \mathrm{mTC}$-amexin $\mathrm{V}$ for repetitive detection of apoptotic tumor response to chemotherapy: an experimental study using a rat cumor model. J Nad Med. 2004:45:309-12.

104. Ke S, Wen X, Wu QP, Wallace S, Charnsangavej C, Stachowiak AM, Srephens CL, Abbruzzese JL, Podoloff DA, Li C. Imaging taxane-induced tumor apoptosis using PEGylated, 111.ln-labeled annexin V.J Nud Med. 2004;45:108-15.

105. Kenerink GJ, Boersma HH, Thimister PW, Hofstra L, Liem \H, Pakbiers MT, Janssen D, Reurelingsperger CP, Heidendal GA. Biodistribution and dosimetry of $99 \mathrm{~m}$ T $\mathrm{C}-\mathrm{BTAP}$ amnexin-V in humans. Eur J Nad Med. 2001;28:1373.8.

106. Petrovsky A, Schellenberger E, Josephsorn L, Weissleder R, Bogdlanov A, Jn. Near-infrared Auroscent imaging of tumor apoptosis. Cancer Res. 2003;63:1936 42 . 
107. Blankenberg FG, Robbins RC, Stoot JH, Vriens PW, Berry GJ, Tait JF, Strauss HW. Radionuclide imaging of acute lung transplant rejection with annexin V. Chest. 2000;1 17:834-40.

108. Ogura Y, Krams SM, Martinez OM, Kopiwoda S, Higgins JP, Esquivel CO, Strauss HW, Tat JF, Blankenberg FG. Radiolabeled annexin V imaging: diagnosis of allograft rejection in an experimental rodent model of liver transplantation. Radiology. 2000:214:795-800.

109. D'Arceuil H, Rhine W, de Crespigny A, Yenari M, Tait JF, Strauss WH, Engellhorn T, Kastrup A, Moseley M. Blankenberg FG. $99 \mathrm{mT}$ cannexin V imaging of neonatal hypoxic brain injury, Stroke. 2000;31:2692-700.

110. Post AM, Katsikis PD, Tait JF, Geaghan SM, Strauss HW, Blankenberg FG. Imaging cell death with radiolabeled annexin $V$ in an experimental model of theumatoid arthriris. $J$ Nud Med. 2002;43:1359-65.

111. Tokita N, Hasegawa S, Maruyama K, Izumi T, Blankenberg FG, Tair JF, Strauss HW, Nishimura T. 99 m Te-Hynic-annexin $V$ imaging to evaluate inflammation and apoptosis in rats with autoimmune nyocarditis. Eur J Nud Med Mol Imaging. 2003;30:232-8.

112. Peker C, Sarda-Mantel L, Loisean P, Rouzet F, Nazneen L, Marter G. Vrigneaud JM, Meulemans A, Samon G, Michel JB, Le Guludec D. Imaging apoptosis with (99m)To. annexin- $W$ in experimental subacute myocarditis. J Nud Med. 2004;45:1081-6.

113. Narula J, Zaret BL. Noninwasive detection of cell death: from rracking epitaphs to counting coffins. J Nud Cardiol, 2002;9:554-60.

114. Reutelingsperger CP, Dumont E. Thimister $\mathrm{PW}$, wan Genderen $H$, Kenis $H$, van de Eijnde, S, Heidendal G, Hofstra L. Visuallization of cell death in wiwo with the annexin A5 imaging prococol. I Inmwol Metbods, 2002;265:123-32.

115. Kown MH, Strauss HW, Blankenberg FG, Berry G], Stafford-Cecil S, Tait JF, Goris ML, Robbins RC. In wiwo imaging of acute cardiac rejection in human patients using. (99m) technetium labeled annexin V. Am J Transplayt. 2001;1:270-7.

116. Zhatng J, McDonald KM. Bioenergetic consequences of left ventricular remodeling. Circulation. 1995;92:1011-9.

117. Gomez AM, Valdivia HH, Cheng H, Lederer MR, Santana LF, Cannell MB, McCune SA, Alschuld RA, Lederer WJ. Defective excitation-contraction coupling in experimental cardiac hypertophy and heart failure. Science. 1997;276:800-6.

118. Kietselaer BL, Narula J, Heidendal GA, Boersma HH, Wolters SL, Liem IH, Hofstra L. Noninvasive detection of apoptosis during worsening of human dilated cardionyopathy using $99 \mathrm{~m}$ Technerium-labelled annexin A5 [abstract]. Circularion: 2004;110:436.

119. Kolodgie FD, Narula J, Burke AP, Haider N, Farb A, Hui-Liang Y, Smialek J, Virmani R. Localization of apoptotic mactophages at the site of plaque tupture in sudden coronary death. Am J Patbol. 2000:157:1259-68.

120. Kolodgie FD, Perrov A, Virmani R, Narula N, Verjans JW, Weber DK, Harrung D, Steinmetz N, Vanderheyden JL, Vannan MA, Gold HK, Reurelingsperger CP, Hofstra L. Narula J. Targeting of apoptotic macrophages and experimental atheroma with radiolabeled annexin $V$ : a technique with potential for noninvasive imaging of vulnerable plaque. Circulation. 2003;108:3134.9.

121. Kierselaer BL, Reutelingsperger CP, Heidendal GA, Daemen MJ, Mess WH, Hofstra L, Narnala J. Noninvasive detection of plaque instability with use of radiolabeled annexin $A 5$ in parients with carotidartery atherosclerosis. N Engl J Med. 2004;350:1472-3.

122. Reynen K. Frequency of primary tumors of the heart. Am J Cardiol. 1996:77:107.

123. Blondenu P. Primary cardiac tumors- French studies of 533 cases. Thorac Cardiovasc Surg. $1990 ; 38$ Suppl 2:192-5.

124. McAllister HA. Tumors of the cardiovascular system. Hartman WH, ed. Atas of tumor pathology. Second series ed. Wasbington DC: Armed Forces institute of pathology, 1978. 


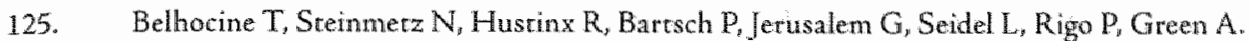
Increased uptake of the apoptosis-imaging agent ( $99 \mathrm{~m}$ ) Tc recombinant human Annexin $V$ in human tumors after one course of chemotherapy as a predictor of tumor response and parient prognosis. Clin Comcer Res. 2002;8:2766-74.

126. Kartachowa M. Haas RL, Olmos RA, Hoebers FJ, wan Zandwijk N, Verhej M. In vivo imaging of apoptosis by $99 \mathrm{~m} T \mathrm{~T}-\mathrm{A}$ nnexin $\mathrm{V}$ scintigraphy: visual analysis in relation to treatment response. Radiother Oncol. 2004;72:333-9.

127. MeLaughlin R, Kelly CJ, Kay E. Bouchier-Hayes D. The role of apoptoric cell death in cardiowascular disease. Ir J Med Sci. 2001;170:132-40.

128. Mattson MP, Duan W, Pedersen WA, Culmsee C. Neurodegenerative disorders and ischennic brain diseases. Apoptosis. 2001;6:69-81.

129. Kerr JF, Winterford CM, Harmon BV. Apoptosis. Its significance in cancer and cancer therapy. Cancer. 1994;73:2013-26.

130. Tait JF, Gibson. D, Fujikawa K. Phospholipid binding properties of human placenral anticoagulant protein-1, a member of the lipocortin family.J Biol Chew. 1989;264:7944-9.

131. Blackwood RA, Ernst JD. Characterization of $\mathrm{Ca} 2(+)$-dependent phospholipid binding, vesicle aggregation and membrane fusion by annexins. Biochem J. 1990;266:195-200.

132. Blankemberg FG, Tait JF, Strauss HW. Apoprotic cell dearh: its implications for imaging in the next millennium. EarJ Nad Med. 2000;27:359-67.

133. van den Eijnde SM, Lips J, Boshart L, Vermeij-Keers C, Marani E, Reutelingsperger CP, De Zeeuw CI. Spatiotemporal distribution of dying neurons during early nouse development. Eur J Newrosci. 1999;11:712-24.

134. Proost JI- Meijer. DK. MW/Pharm, an integrated software package for drug dosage regimen calculation and therapeuric drug monitoring. Comput Biol Med* 1992;22:155-63.

135. Prince JR, Bancroft S, Dukstein WG. Pharmacokinetics of pertechnetate administered after pretreatment with $400 \mathrm{mg}$ of potassium perchlorate: concise communication. J Nwd Med. 1980;21:763-6.

136. Kukis DL, DeNardo GL, DeNardo SJ, Mirick GR, Miers LA, Greiner DP, Meares CF. Effect of the extent of chelare substiturion on the immunoreactivicy and biodistribution of 2IT-BAT-Lym-1 immunoconjugares. Cancer Res. 1995;55:878-84.

137. Buijs WC, Siegel JA, Boerman OC, Corstens FH. Absolute organ acrivity estimated by five different methods of background correction. I Nud Med. 1998;39:2167.72.

138. Stabin MG. MIRDOSE: personal computer sofware for internal dose assesment in nuclear medicine. J Nud Med. 1996;37:538-46.

139. Siegel JA. The effect of source size on the buildup factor ealculation of absolute volume.J Nwal Med. 1985;26:1319-22.

140. Sicgel JA, Thomas SR, Stubls JB, Stabin MG, Hays MT, Konal KF, Robertson JS, Howell RW, Wessels BW, Fisher DR, Weber DA, Brill AB. MIRD panphler no. 16 : Techniques for quantitative radiopharmaceurical biodistribution data acquisition and analysis for use in human radiation dose estimates. I Nut Med. 1999;40:37S-61S.

141. Buijs WC, Oyen WJ, Dams ET, Boerman OC, Siegel JA, Chaessens RA, warn der Meer JW, Corstens. FH. Dynamic distribution and dosimetric evaluation of human non-specific immunoglobulin G labelled with 111In or 99Tem. Nucl Med Commun. 1998;19:743-51.

142. Clontier RJ, Smith SA, Watson EE, Snyder WS, Warner GG. Dose to the fetus from radionuclides in the bladder. Hewlth Phys. 1973;25:147-61.

143. Shen S, DeNardo GL, Sgouros G, O'Donnell RT, DeNardo SJ. Practical deremination of patient-specific marrow dose using radioactiwity concentration in blood and body. I Nud Med. 1999;40:2102-6.

144. Anonymous. ICRP Publication 80. Radiation dose to patients frow radiopharmatceuticals. Addendum to ICRP publication 53: Oxford: Perganon Press; 1998. 
145. Chen M, Won Df, Krajewski S, Gottlieb RA. Calpain and mitochondria in ischemial reperfusion injury. J Biol Chem. 2002;277:29181-6.

146. Chien KR. Genomic circuits and the integrative biology of cardiac diseases. Nature. 2000;407:227-32.

147. Narula J, Pandey P, Arbustini E, Haider N, Narula N, Kolodgie FD, Dal Bello B, Semigran MJ, Bielsa-Masdeu A, Dec GW, Israels $S$, Ballester M, Virmani R, Saxena

$S_{\text {, }}$ Kharbanda $S$. A poptosis in heart failure: release of cy tochrome $c$ from mitochondria and activation of caspase-3 in human cardiomyopathy. Proc Natl Arad Sci US A. 1999;96:8144-9.

148. Kang PM, Izumo S. A poptosis and heart failure: A critical review of the literature. Circ

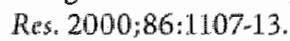

149. Bialik S, Geenen DL, Sasson IE, Cheng R, Horner JW, Evans SM, Lord EM, Koch CJ, Kitsis RN, Myocyte apoptosis during acure myocardial infarction in the mouse localizes to hy poxic regions but occurs independently of p53.J Clin Invest. 1997;100:1363-72.

150. Kumar D, Jugdut BI. Apoptosis and oxidants in the heart. J Lab Clin Med. 2003;142:288-97.

151. Holly TA, Drincic A, Byun Y, Nakamura S, Harris K. Klocke FJ, Cryns VL. Caspase inhibition reduces myocyte cell death induced by myocardial ischemia and reperfusion in vivo. J Mal Cell Cardiol. 1999;31:1709-15.

152. Huang Q , Radinowic S, Rezaiefar P, Black SC. In wiwo myocardial infarct size reduction by a caspase inhibitor administered after the onset of ischemia. Eur Phamacol. $2000 ; 402 \div 139-42$.

153. Condorelli G, Roncarati R, Ross J, Jr., Pisani A, Stassi G, Todaro M, Trocha S, Drusco A, Gu Y, Russo MA, Frati G, Jones SP, Lefer DJ, Napoli C, Croce CM. Heart-targeted overexpression of caspase 3 in mice increases infarce size and depresses cardiac function. Proc Natl Acad Sci U S A. 2001;98:9977-82.

154. Chen Z, Chua CC, Ho YS, Hamdy RC, Chua BH. Overexpression of Bci-2 attenuates apoptosis and protects against myocardial I/R injury in transgenic mice. $A m J P b y s i o l$ Heart Cinc Physiol. 2001;280:H2313-20.

155. Chen M, He H, Zhan S, Krajewski S, Reed JC, Gottlieb RA. Bid is cleaved by calpain to an active fragment in vitro and during myocardial ischemia/treperfusion. J Biol Chem. 2001;276:30724-8.

156. Kreuter M, Langer C, Kerklhoff C, Reddanna P, Kania AL, Maddika S, Chlichlia K, Bui TN, Los M. Stroke, mocardial infarction, acute and chronic inflammatory diseases: caspases and other apoptotic molecules as targets for drug development. Arch Innmunol Ther Exp (Warsz). 2004:52:141-55.

157. Langevizz P. Livnet A, Bank I, Pras M. Benefits and risks of minocycline in theumatoid arthritis. Drug Suf 2000;22:405-14.

158. Pisersky DS, Se Clair EW. Progress in the treatment of rheumatoid arthritis. Jama. 2001; 286:2787-90.

159. Arvin KL, Han BH, Du Y, Lin SZ, Paul SM, Holtzman DM. Minocycline markedly prorects the neonatal brain against hypoxic-ischemic injury. Am Neurol. 2002;52:54-61.

160. Bantubungi K, Jacquard C, Greco A, Pincor A, Chtarto A, Tai K, Galas MC, Tenenbaum I., Deglon N, Popoli P, Minghetri L, Brouillet E, Brotchi J, Levivier M. Schiffmann SN, Blum D. Minocycline in phenotypic models of Huntington's disease. Neurobid Dis. $2005: 18: 206-17$

161. Du Y, Ma Z, Lin S, Dodel RC, Gao F, Bales KR. Triathou LC, Chernet E, Perry KW, Nelson DL, Luecke S, Phebus LA, Bymaster FP, Puul SM. Minocycline prevents nigrostriatal dopaminergic neurodegenetation in the MPTP model of Parkinson's disease. Proc Natl Acad Sci US A. 2001;98:14669-74.

162. Scarabelli TM, Stephanou A, Pasini E, GirtiG, Townsend P, Lawrence K, Chen- 


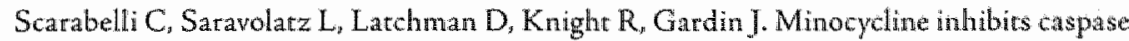
actiwation and reactivation, increases the ratio of XIAP to smac/DIABLO, and reduces the micochondrial leakage of cytochrome $\mathrm{C}$ and snac/DIABLO.I Am Call Cardial. $2004: 43: 865-74$.

163. Narula ], Petrov A, Pak KY, Lister BC, Khaw BA. Very early noninvasive detection of acute experimental nonteperfused myocardial infarction with $99 \mathrm{mi}$ Tc-labeled glucarate. Circulation. 1997;95:1577-84.

164. Freude B, Masters TN, Robicsek F, Fokin A, Kostin S, Zimmermann R, Ulmann $C_{\text {, }}$ Lorenz-Meyer S, Schaper J. Apoptosis is initiated by myocardial ischenia and execured during reperfusion.J Mol Cell Cardiol. 2000;32:197-208.

165. Fliss $\mathrm{H}$, Gatringer D. Apoptosis in ischemic and reperfused rat myocardium, Circ Res. 1996;79:949-56.

166. Gortlieb RA, Burleson. KO, Kloner RA, Babior BM, Engler RL. Reperfusion injury induces apoptosis in rabbit cardiompocytes. J Clin Invest, 1994:94:1621-8.

167. Sarasre A, Pulkki K, Kallajoki M, Henriksen K, Parvinen M, Voipio.Pulkki LM. Apoptosis in human acute myocardial infarction. Circulation. 1997;95:320-3.

168. Itoh $G$. Tamura J, Suzuki M, Suzuki $Y$, Ikeda H, Koike M, Nomura M, Jie T, Ito K. DNA fragmentation of human infareted myocardial cells demonstrated by the nick end labeling method and DNA agarose gel ellectrophoresis. Am J Patbol. 1995;146:1325-31.

169. Brown JM, Attardi LD. The role of apoptosis in cancer development and treatment response. Nat Rev Cancer. 2005;5:23117.

170. O'Dell JR, Blakely KW, Mallek JA, Eckhoff PJ, Leff RD, Wees SJ, Sems KM, Fernandez AM, Palmer WR, Klassen LW, Paulsen GA, Haire CE, Moore GF. Treatment of early seropositive theumatoid arthritis: a two-year, double-blind comparison of minocycline and hydroxpchloroquine. Artbritis Rheum. 2001;44:2235-41.

171. Boersma HH, Dumont EA, Hofstra L, Bennaghmouch A, Stolk LM, Beysens AJ, Kemerink GJ, Heidendal GA, Reutelingsperger CP. Effect of eniporide on the biodistribution of $99 \mathrm{mTC}$-Annexin $\mathrm{A} 5$ after ischemia/ reperfusion of the myocardium.J Nud Med. 2003;44:5 Suppl. S.

172. Subbarayan M, Hafeli UO, Feyes DK, Unnithan J, Emancipator SN, Mukhtar H. A simplified mechod for preparation of $99 \mathrm{~m}$ Tc-annexin $\mathrm{V}$ and its biologic evaluation for in viwo imaging of apoptosis afrer photodynamic therapy. $J$ Nud Med. $2003 ; 44: 650 \times 6$.

173. Reutelingsperger C. Hofstra L, Natula J. Cooking annexin V: a simple 1-pot procedure to destroy its phosplatidylserine-bincling activity. J Nud Med. 2004;45:1098; author reply 1098-9.

174. Tait JF, Smich C, Blankenberg FG. Structural Reguirements for In Viwo Detection of Cell Dearth with 99 mic-Annexin V.J Nad Med. 2005;46:807-15.

175. Boersma HH, Kietselaer BL, Srolk LM, Bennaghmouch A, Hofstra L, Narula J, Heidendal GA, Reutelingsperger CPM. Past, present and future of Annexin A5: from protein discovery to dinical applications. I Nud Med, 2005;46:2035-50.

176. Sarai M, Isobe S, Petrov AD, Yim CB, Boersma HH, Kemerink G], Dirksen A, Hackeng T, Vanderheyden J.L, Hofstra L, Reutelingsperger CPM, Narula J. Noninvasive detection of apoptosis in experimental myocardial infarction with $111 \mathrm{ln}$-labeled annexin A5: possible clinical applications. Submitted.

177. Corsten MF, Hofstra L, Narula J, Reurelingsperger CPM. Counting Heads in the War Against Cancer, Defining the Role of Annexin A5 Inaging in Cancer Treatment and Surveillance. Cancer Res 2006; 66: 1-6. 


\subsection{Summary}

PS-expression on the outer cell membrane is one of the "eat me" signals at the surface of the dying cell. PS-affinity of annexin A.5 forms the basis for its development as a diagnostic tool.

Over the last ten years, annexin A5 has been developed for both in vitro and in wivo use to detect cell death. Annexin A5 was labeled with fluorescent groups, biorine or a radioisotope to visualize its binding to expressed PS on dying cells in vitro and in vivo. The research described in this thesis is based on the cell death detecting properties of annexin $\mathrm{A} 5$. The main goal was to improve molecular imaging with annexin A5. We showed that it is possible to improve quality control of radiolabeled annexin A5, we described its biodistribution and dosimetry and finally showed that the annexin A5 imaging protocol can be used as a tool to evaluate drug treatment in animals during I/R experiments.

Chapter 1 gives an introduction into annexin A5 and shows its properties and challenges. We discussed annexin A5's biological activity as the radiopharmaceutical, pharmacological and biodistribution aspects of annexin A5. Furthermore, clinical relevance, limitations and furure perspectives of this approach of visualizing cell death are reviewed and discussed. Radiolabeled annexin A5 looks promising in becoming a clinically applied radiopharmaceutical with potential applications in cardiology and oncology. Visualization of cell death is important in pathologies such as myocardial infarcrion, atherosclerosis and cancer. Also, it may be developed towards a tool for monitoring cell death inducing or cell death preventing therapies. In addition, the experiences with radiolabeled annexin $A 5$ open novel avenues for drug targering with annexin $\mathrm{A} 5$ as a biological "cruise missile".

The first target to achieve this was to develop a quality control method that made it possible to quickly and accurately assess the biological functionality of radiolabeled annexin A5 for example. We described such a method in Chapter 2. Because of the short half life of the commonly used radioisotope labels it is important to limit the time span between preparation of radiolabeled annexin $A 5$ and its administration in the patient. Therefore methods of quality control that determine the biologically active fraction in the radiolabeled annexin A5 should not only be accurate and precise but also rapid. We reported the development and validation of a rapid, simple assay measuring the biological active fraction of radiolabeled annexin A5. The assay is based on a solid phase of paramagnetic beads that are coated with phospholipids. Annexin A5 binds to these beads with high affinity if PS is present within the phospholipid coar. Furthermore the binding depends on $\mathrm{Ca}^{2+}$-ions and funcrional $\mathrm{Ca}^{2+} /$ phospholipid binding sites of Annexin A5. The bead assay is specific, stability indicating, repeatable and reproducible. Ir allows for determination of the biological active fraction of a radiolabeled annexin A5 preparation, within 25 minutes. We dubbed this assay the ApoCorrect ${ }^{\text {rat }}$ assay. 
In Chapters 3 and 4, a thorough description and analysis of annexin A5's labeling methods, biodistribution, pharmacokinetics and imaging properties was given.

In Chapter 3 two radioisotope conjugation methods have been compared with respect to their biodistribution and imaging properties. To evaluate the potential of radiolabeled annexin A5 we studied the pharmacokinetics and imaging properties of two investigational radiopharmaceuticals, Imino-annexin A5 and BTAP-annexin A5. Plasma concentrarions of ${ }^{99 \mathrm{~m}} \mathrm{TC}$-radioactivity and the annexin $\mathrm{A} 5$ antigen were quantified up to $4 \mathrm{~h}$ after administration. Furthermore, for BTAP-annexin A5 the stability in plasma was assessed. Endogenous annexin A5 levels were also measured in a control group of patients with myocardial infarction. These data were compared to the annexin A5 levels found in the treated subjects. Pharmacokinetic parameters were derived by non-linear regression analysis. The biodistribution of radioactivity was derived from conjugate anterior and posterior views obtained with a dual head gamma camera, applying corrections for attenuation and scatter.

The kinetics of radioactivity in plasma could be described by a two compartment model. Both preparations had similar half-lives $\left(t_{w_{2}, \alpha} \approx 15 \mathrm{~min}, \mathrm{t}_{\mathrm{ts}, \mathrm{B}} \approx 4 \mathrm{~h}\right)$, but showed a different distribution of the radioactivity over both components. The BTAP-annexin A 5 complex was stable in plasma up to at least $20 \mathrm{~h}$ after injection. The endogenous level of annexin A5 in the control group showed considerable fuctuations, as did the total annexin A5 level in the patients receiving technetium labeled annexin A5. Both radiopharmaceuticals accumulated in the kidney and the liver, and later in the gut. BTAP-annexin A5 was excreted significantly faster than Imino-annexin A5. Furthermore, we showed the feasibility of cell death imaging in parients suffering from myocardial infarction as well as tumors of both head and neck region and the extremities. Due to the poor biodistribution of both conjugation methods, however, imaging in liver, kidney, spleen and abdomen is not possible. So, new labeling modalities are required to enable imaging with annexin $A 5$ in these areas.

In Chapter 4 the biodistribution and the associated radiation dose of BTAP. annexin A5 has been studied. Organ uptake was estimated after correction for body background activity, attenuation and scatter. Residence times were calculated from the dynamic and whole-body studies. Furthermore organ doses and effective dose were determined. It was found that the radioactivity accumulated mostly in the kidneys and the liver. Uptake in the target tissues (lymphomas or heart) was negligible from a dosimetric point of view. By extrapolation of data, one finds that approximately one quarter of the injected dose will be excreted in the feces, and all other excretion will take place in the urine. BTAP-annexin A5 has a high uptake in the kidneys and liver, even higher than that found for the previously studied Iminoannexin A5. The biological half-life is shorter, however, but still long compared with the physical half-life of $9 \% \mathrm{~m} T \mathrm{~T}$. The faster appearance of activity in the intestines may preclude imaging of apoptosis in the abdomen. The effective dose is within the lower range of values reported for typical ${ }^{99_{m}} \mathrm{Tc}$ compounds. 
Finally, we showed how a treatment modality can be assessed using radiolabelled annexin $\mathrm{A} 5$. In Chapter 5 , the cell dearh inhibitory effect of minocycline has been evaluated. Minocycline is a commonly used antibiotic that exerts anti-apoptotic activity. It was tested in two models of myocardial I/R injury, using fluorescent Alexa568-annexin A5 as well as HYNIC-annexin A5 to determine the change in the extent of myocardial damage. Firstly, I/R injury was produced in a mouse model. Three of 6 Swiss mice received minocycline treatment at reperfusion. Then, optical imaging using Alexa568-annexin A5 was performed. Furthermore, we tested the hypothesis in a rabbit I/R model. Here, HYNIC-annexin A5 was injected. ${ }^{201}$ TI was injected shortly before sacrifice i.v. to define the myocardium at risk by tightening the ligature again. Ex vivo dual isotope imaging was performed on the heart to define the areas under risk and clamage. Gamma counting of the fractionated heart was performed to assess myocardial damage. Three of the 6 rabbits received minocycline at reperfusion. We performed annexin A5 imaging in both mice and rabbits treated without and with minocycline. Treated mice demonstrated a significant lower uptake of Alexa568-annexin A5. Minocycline treated rabbits showed a significant reduction in \% injected dose per gram uptake of HYNIC-annexin A5 in the area at risk as compated to control animals.

Therefore, we conclude that administration of minocycline at reperfusion rescues cardiomyocytes from apoptoric insult induced by $I / R$. These results should encourage the use of minocycline in clinical $I / R$ studies. 


\section{Final conclusions.}

By performing the research in this thesis, more information was gathered about annexin A5's properties as a radiopharmaceutical and imaging tracer. We developed a biofunctionality assay to achieve a better quality control on the PS-binding properties of the molecule before administration to the patient. Furthermore, we described the pharmacokinetics, biodistribution and imaging properties of two radiotracers containing annexin A5. The latter gave us more information about its imaging and biodistribution pitfalls. This, in order to aid the development of better annexin A5 imaging probes in the future. Finally, it has been shown how the annexin $\mathrm{A} 5 \mathrm{imaging}$ protocol can be used as a tool for treatment evaluation. We assessed minocycline cell dearh inhibition in myocardial I/R injury by annexin A5 imaging. This methology is already being used successfully for treatment evaluation in radiation therapy. Therefore, there is a need for further clinical research to establish therapy evaluation using annexin A5. 


\subsection{List of Publications}

\section{At Annexin A5-related papers}

Boersma HH. "W door SPECI-beeldworming Pharm Weekbl 2001; 136: 106-111

Boersma HIH, Liem IH, Kemerink GJ. Thimister PWL, Hofstra L, Stolk LML, van Heerde WL, Pakbiers M-Th W, Janssen D, Beysens AJ, Reutelingsperger CPM, Heidendal GAK. Comparison between human pharmacokinetics and imaging properties of two conjugation methods for ${ }^{99 n} \mathrm{~T}$-Annexin $\mathrm{A} 5$. Br J Radiol 2003; 76:553-60.

Boetsma HH, Stolk LML, Kenis H, Deckers NM, Vanderheyden J-L, Hofstra L, Heidendal GAK, Reurelingsperger CPM. The A poCorrect assay: a novel, rapid method to determine the biological functionality of radiolabeled and fuorescent Annexin A5. Analytical Biochemistry $2004 ; 327: 126-34$.

Boersma HH, Kietselaer BJLH, Stolk LML, Bennaghmouch A, Hofstra L, Narula J, Heidendal GAK, Reutelingsperger CPM. Past, present and future of Annexin A5: from protein discowery to clinical applications. J Nucl Med 2005; 46: 2035-50

Boersma HH, Petrov AD, Wolters SL, Bitsch N, Sarai M, Isobe S, Bennaghmouch A, Kietselaer BL, Stolk LML, Teule GJJ, Heidendal GAK. Hofstra L, Reutelingsperger CPM, Narula ]. Minocycline inhibits cell death in vivo in myocardial ischemia and subsequent reperfusion. Submitted.

Boersma HH, Dumont EA, Verjans JW, Kietselaer BLJH, Hofstra L, Bennaghmouch A, Kemerink GJ, Stolk LML, Beysens T, Heidendal GAK, Narula J, Reutelingsperger CPM. Effect of eniporide on the biodistribution of ${ }^{9 \% n}$ Tc-annexin A5 in mice undergoing myocardial ischemia and subsequent reperfusion. In preparation.

Kemerink G], Boersma HH, Thimister PWL, Hofstra L, Liem IH, Pakbiers M-Th, Janssen D, Reutelingsperger CPM, Heidendal GAK. Biodistribution and dosimetry of 99mT-BTAP-annexin $V$ in humans. Eur J Nud Med 2001; 28: 1373.8.

Corsten MF, Kietselaer BLJH, Bennaghmouch A, Reutelingsperger CPM, Boersma HH, Hofstra L. A Molecular wiew of apoptosis with annexin A5. A focus on cardiovascular disease. Annexins 2005; 2; 25-34.

Hofstra L, Liem IH, Dumont EA, Boersma HH, van Heerde WL, Doevendans PA, DeMuinck E, Wellens HJJ, Kemerink GJ, Reutelingsperger CPM, Heidendal GA. Visualisation of apoptosis in vivo in patients with an acute myocatdial infarction. Lancet $2000 ; 356 ; 209-12$. 
Hofstra L, Dumont EA, Thimister PWL, Heidendal GAK, de Bruine AP, Elenbaas TWO, Boersma $H H$, van Heerde WL, Reutelingsperger CPM. In vivo detection of apoptosis in an intra-cardiac tumor. JAMA 2001; 285: 1841-2.

Kemerink G], Liem IH, Hofstra L, Boersma HH, Buijs WCAM, Reutelingsperger CPM, Heidendal, GA. Patient dosimetry of intravenously administered ${ }^{99 m}$ TC-labeled annexin V. JNud Med 2001; $42: 382-7$.

Kierselaer BLH], Thimister PWL, Reutelingsperger CPM, Heidendal GAK, Dumont EAWJ, Boersma HH, Hofstra L. Molecular imaging of cell death in intra-cardiac tumors. A new approach to differential diagnosis in cardiac tumors.

The Netherlands Heart Journal 2002; 10; 312-6.

Kietselaer BJLH, Reutelingsperger CPM, Boersma HH, Heidendal GAK, Liem IH, Wellens H.J, Hofstra L, Narula J. Non-inwasive Assessment of Programmed Cell Death in Patients with Recent Worsening of Heart Failure. Submitted.

Sarai M, Isobe $S$, Petrov AD, Yim CB, Boersma HH, Kemerink GJ, Dirksen A, Hackeng T, Vanderheyden J-L, Hofstra L, Reutelingsperger CPM, Narula J.

Noninvasive detection of apoptosis in experimental myocardial infarction with "In-labeled annexin $A 5$ : possible clinical applications. Submitted.

Thimister PWL, Hofstra L, Liem IH, Boersma HH, Kemerink GJ, Reutelingsperger CPM, Heidendal GAK. In wivo detection of cell death in the area at risk in acute myocardial infarction. J Nucl Med. 2003:44:391-6.

Wolters SL. Appleby S, Kietselaer BL, Lutgens E, Boersma HH, Kubaska S, Daemen M, Narula $N$, Tordoir JH Teule J, Fujitani R, Milne N, Reutelingsperger CP, Narula J, Hofstra L. Molecular imaging of unstable atherosclerotic lesions in patients undergoing carotid endareterctomy. Submitted. 


\section{B: Other publications}

Boersma HH, Woerdenbag H.J, Bauer J, Scheirhauer W, Kampinga HH, Konings AWT. Interaction between the cytostatic effects of quercetin and 5-fluorouracil in two human colorectal cancer cell lines. Phytomedicine 1994; 1: 239-44.

Boersma $\mathrm{HH}$, van Hulst W. Zorg op mat door de openbare apotheek: invoeren en protocollering van doseerdoosdistributie. Pharm Weekbl 1996; 131: 1169-73.

Boersma HH, Groothuijsen HJG, Stolk LML, Beysens AJMM. "Goed houdbaar onder normale omstandigheden". Stabiliteitsonderzoek van natrium-p-aminohippuratinjectievloeistoffen. Pharm Weekbl 1999; 134: 1444-8.

Boersma HH, Wensing JW, Kho TL, de Brauw LM, Liem IH, wan Ktoonenburgh MPJG. Transient enhanced uptake of ${ }^{123}$ I-Metaiodobenzylguanidine in the contralateral adrenal region after resection of an adrenal pheochromocytoma. New EngJ Med 2000; 342:1450-1

Boersma HH, Wensing JW, Kho TL, de Brauw LM, Liem IH, van Kroonenburgh MPJG. Compensatory Uptake of ${ }^{123}$ I-MTBG in the Contralateral Adrenal after Removal of a Pheochtomocytoma. Clin Nucl Med 2002; 27:113-6.

Boersma $\mathrm{HH}$, Tromp SC, Hofsrra L, Narula J. Stem Cell Tracking: On reversing the silence of the lambs...+. I Nud Med 2005, 46:200-3.

Co-Author of: Het Palliatief Formularium (Palliative Formulary), van den Beuken-van Everdingen $\mathrm{MJH}$, Janssen-Jongen MLE, Eck MAMF (eds). Bohn, Staffleu \& Van Lochum 2003 ( $1^{\text {st }}$ edition), $2004\left(2^{\text {ad }} \mathrm{ed}\right.$ )

van Merode GG, Schoenmakers M, Groothuis $\mathrm{S}$, Boersma HH. Simulation studies and the alignment of interests. Health Care Management Science 2002; 5: 97-102.

van Merode Fi Schoenmakers M, Boersma HH, Groothuis S. Ontwerp van zorgprocessen. In: A. van Raak, A. Paulus (red.) Onderzoek doen in de zorg. Elsevier gezondheidszorg, Maarssen, 2002: 89-107.

Postema EJ, Boersma HH. Radioimmunotherapy as a Treatment Modality for NonHodgkin's Lymphoma. Drugs of the Future 2004, 29: 95-100.

Stolk LML, Boersma HH."Innemen 2 uur voor of na een ment met spruitjes". Voeding en het metabolisme van geneesmiddelen. Pharm Weekbl 1999; 134: 1254-9.

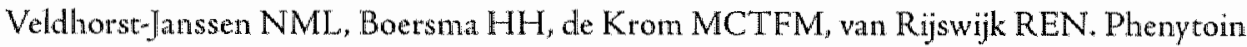
interacts with a regafur/folinic acid chemotherapy regimen. Br J Cancer 2004; 90: 745. 
Weikamp S, Boersma HH, van Suijlekom JA, Stolk LML. Bupivacaïne en baclofen in én injectieoplossing: de stabiliteir van de combinatie onderzocht.

Pharm Weekbl 2002; 137: 1358-60. 


\subsection{List of Abbreviations}

\begin{tabular}{|c|c|c|}
\hline AIF & $=$ & apoptosis inducing protein factor \\
\hline ANOVA & $=$ & Analysis of Variance \\
\hline ATP & $=$ & adenosine triphosphate \\
\hline BTAP-annexin A5 & $=$ & $\begin{array}{l}\text { 4,5-bis(thioacetamido) pentanoyl)-annexin A5, } \\
{ }^{9 \mathrm{~m}} \mathrm{TC}_{\mathrm{C}} \mathrm{B} \text { TAP-annexin A5. }\end{array}$ \\
\hline Caspase & $=$ & Cystein aspartate specific protease \\
\hline ¿FLIP & $=$ & cellular FLICE Inhibitory Protein \\
\hline Chol & $=$ & Cholesterol \\
\hline $\mathrm{CT}$ & $=$ & computer tomography \\
\hline $\mathrm{CV}$ & $=$ & coefficient of variance \\
\hline DTPA & $=$ & DiethyleneTriamine Penta Acetate \\
\hline EC-annexin A5 & $=$ & ethylenedicysteine-annexin A5 \\
\hline EDDA & $=$ & ethylenediamine diacetic acid \\
\hline EDTA & $=$ & erhylenediaminetetraacetic acid \\
\hline EGTA & $=$ & $\begin{array}{l}\text { ethylene glycol bis(2-aminoethyl ether) }-\mathrm{N}, \mathrm{N}, \mathrm{N}^{\prime} \mathrm{N}^{\prime} \text { - } \\
\text { tetraacetic acid }\end{array}$ \\
\hline ELISA & $=$ & Enzyme Linked Immunosorbent Assay \\
\hline FITC & $=$ & Fluorescein Isothiocyanate \\
\hline FPLC & $=$ & Fast Protein Liquid Chromatography \\
\hline GTP & $=$ & guanosine triphosphate \\
\hline $\mathrm{H} \& \mathrm{E}$ & $=$ & Hematoxylin and Eosin \\
\hline HEPES & $=$ & 4-(2-hydroxyethyl)-1-piperazineethanesulfonic acid \\
\hline HYNIC-annexin A5 & $=$ & $\begin{array}{l}\text { hydrazino-nicotinamide-annexin A5, } \\
\text { 99m TC-HYNIC-annexin A5 }\end{array}$ \\
\hline $\mathrm{I} / \mathrm{R}$ & $=$ & ischemia and reperfusion injury \\
\hline Imino-annexin $\mathrm{A} 5$ & $=$ & ${ }^{99 m}$ Tc-(n-1-imino-4-mercaptobutyl)-annexin A5 \\
\hline 1D & $=$ & injected dose \\
\hline $\mathrm{IC}_{50}$ & $=$ & $\begin{array}{l}\text { the concentration at which } 50 \% \text { of the inhibitory } \\
\text { effect is observed }\end{array}$ \\
\hline ITLC, TLC & $=$ & Thin Layer Chromatography \\
\hline LAD & $=$ & Left Anterior Descending coronary artery \\
\hline M1234 & $=$ & the non-PS binding mutant of annexin A5 \\
\hline MIBI & $=$ & Ny \\
\hline MRI & $=$ & magnetic resonance imaging \\
\hline $\mathrm{PC}$ & $=$ & Phosphatidyl choline \\
\hline $\mathrm{PCD}$ & $=$ & programmed cell death \\
\hline $\mathrm{PE}$ & $=$ & phosphatidyl ethanolamine \\
\hline PEG & $=$ & polyethyleneglycol \\
\hline PET & $=$ & Positron Emission Tomography \\
\hline
\end{tabular}


PI

PLA,

PP4

PS

PTCA

QC

RCP

ROS

SPECT

TLC

TNF

TRAIL

TUNEL

$\mathrm{VAC}-\alpha$
$=$ propidium iodide

$=$ Phospholipase $\mathrm{A}_{2}$ enzymes

$=$ placental protein 4

$=$ phosphatidyl serine

$=$ Percutaneous 'Transluminal Coronary Angioplasty

$=$ Quality Control

$=$ Radiochemical purity

$=$ Reactive Oxygen Species

$=$ Single Photon Emission Tomography

$=$ Thin Layer Chromatography

$=$ Tumor Necrosis Factor

$=$ TNF-related apoptosis inducing ligand

$=$ Terminal deoxynUdleotidyl transferase deoxyuridine triphosphate Nick-End Labeling

= vascular anticoagulant protein alpha 


\subsection{Samenvatting}

Als een cel de vetachtige stof PS (fosfatidylserine) naar de buitenkant van de celmembraan brengt, is dit voor de omringende cellen een teken dat de cel gaat sterven. Sommige witte bloedcellen (macrofagen) worden met dit signaal opgeroepen om de betreffende cel op te eten. Het lichaamseigen eiwit annexine A5 kan aan PS binden. Annexine A5 kan gekoppeld worden aan een fluorescerende groep of een radioactief isotoop. Het onderzoek in dit proefschrift is gebaseerd op de hierboven beschreven eigenschap van annexine $\mathrm{A} 5$ om celdood te kunnen opsporen.

Eerder al kon met behulp van dit proces een methode ontwikkeld worden om celdood in gekweekte cellen te meten. De fuorescentie of radioactiviteit wordt hierbij omgezet in een meetbaat signaal. Na wat aanpassingen kon deze methode ook toegepast worden in proefdieren en in de mens. In dit proefschrift is gebruik gemaakt van deze methode.

Het doel van de uitgevoerde experimenten was het verbeteren van bestaande methoden om met radioactief annexine A5 celdood zichtbaar te maken. We hebben allereerst een verbeterde kwaliteitscontrolemethode ontwikkeld. Verder is de verdeling van het eiwit in het lichaam en de radioactieve stralingsbelasting die het radioactieve annexine $\mathrm{A} 5$ tot gevolg heeft, onderzocht. Daarmee werd ook aangetoond dat radioactief annexine in de mens celdoodprocessen bij zowel kanker als hart- en vaatziekten zichtbaar kan maken. Ten slotte hebben we onderzocht hoe annexine A5 gebruikt kan worden voor het beoordelen van de werking van een celdoodremmend geneesmiddel.

Bij de in dit proefschrift beschreven experimenten hebben we zowel gebruik gemaakt van radioactief als fluorescerend annexine A5. Met een gammacamera zijn de radioactieve signalen omgezet in een zichtbaar beeld, terwijl voor de fluorescentieopnamen gebruik gemaakt werd van een fluorescentiemicroscoop om het fluorescente annexine A5 te laten zien.

Hoofdstuk 1 behandelt de eigenschappen van annexine A5, en laat zien welke mogelijkheden het eiwit biedt voor onderzoek. Hierbij worden de biologische eigenschappen, de werking van het eiwit in het lichaam en de verdeling van annexine A5 in het lichaam beschreven. Verder is ingegaan op de manieren waarop annexine A5 in het lichaam zichtbaar gemaakt kan worden, en welke gevolgen het gebruik van de methode heeft voor beslissingen van de arts voor de behandeling van een pariënt. Daarnaast zijn de beperkingen en de relevantie van de methode besproken. We denken dat radioactief annexine A5 geschikt is om als standaardmethode gebruikt te worden voor het meten van celdoodprocessen bij patiënten met bijvoorbeeld kanker of hart- en vaatziekten. De methode kan nier alleen gebruikt worden voor her meten van celdood bij een bepaalde ziekte, maar ook om de behandelingseffecten van geneesniddelen met invloed op de celdood te kunnen bepalen. Verder zou annexine 
A5 een mogelijkheid kunnen bieden om als een soort transportstof te fungeren en een geneesmiddel af te leveren op een plaars waat celdood optreedt. In dat geval kunnen we spreken van een biologische 'kruisraket'.

Hereerste doel in dit proefschrift was het realiseren van een $k$ waliteitscontrolemethode om snel en accuraat de biologische kwaliteir van radioactief of fluorescent annexine A5 te kunnen meten. Dit wordt beschreven in Hoofdstuk 2. Gewoonlijk hebben radioactieve stoffen die in de mens worden toegepast een heel korte halfwaardetijd (dir is de rijd waarin de helft van de radioactiviteit vervalt). Er is hierdoor maat weinig tijd voor de kwaliteitscontrole van het radioactief annexine $\mathrm{A} 5$ omdat het al snel aan een patiënt toegediend moet worden. We hebben aan deze eis voldaan door een simpele, snelle methode te ontwikkelen die nauwkeurig de hoeveelheid biologisch actief annexine $\mathrm{A} 5$ in de oplossing kan vaststellen. Voor de meting werden microscopisch kleine ijzeren bolletjes gebruikt die voorzien zijn van een PS-bevattend laagje. Deze bolletjes werden gemengd met een oplossing met daarin radioactief annexine A5. Het eiwit hechtte zich aan de bolletjes in anwezigheid van calcium. Met een magneet konden de bolletjes na 10 minuten gescheiden worden van de oplossing. Door de oplossing te scheiden van de bolletjes kon de hoeveelheid radioactiviteit in de bolletjes worden bepaald en daarmee de anwezigheid van annexine A5 erop. Eventuele onzuiverheden (bijvoorbeeld radioactiviteit die niet aan annexine A5 gebonden is) kon worden opgespoord door de van de bolletjes gescheiden oplossing te meten. We hebben deze methode uitgebreid getest. Daarbij werd aangetoond dat $z e$ in staat is onzuiverheden te registreren, en dat de bepaling nauwkeurig te herhalen is. Verder was het mogelijk iets te zeggen over het PS-bindend vermogen van het gemeten annexine A5. Wanneer we bijvoorbeeld het eiwit kapot maakten bij $60^{\circ} \mathrm{C}$ of in zuur, dan konden we vaststellen, dat de binding aan de bolletjes minder werd. Met de bolletjesmethode is het mogelijk om in 25 minuten het bindend vermogen van annexine $\mathrm{A} 5$ aan $\mathrm{PS}$ te bepalen.

In de Hoofdstukken 3 en 4 worde een uitgebreide beschrijving gegeven van de verdeling van radioactief annexine $A 5$ in het lichaam van patiënten en proefpersonen. Radioactief annexine A5 wordt met een injectie in een bloedwat aan de patiënt toegediend. Ook wordt een vergelijking gemaakt tussen twee methoden die gebruikt zijn om annexine A5 radioactief te maken. Ten slotte wordt een indruk gegeven van de mogelijkheden die het eiwit biedt om beelden te maken van een harrinfarct en verschillende vormen van kanker.

In Hoofdstuk 3 worden twee methoden die aanvankelijk gebruikt zijn om annexine A5 radioactief te maken met elkaar vergeleken. We hebben hierbij de verdeling in het lichaam van patiënten en een proefpersoon onderzocht. Daarnaast wordt een indruk gegeven van de verschillende mogelijkheden van beide methoden om opnamen van celdoodgebieden bij patiënten te maken. Om het gedrag van annexine $\mathrm{A} 5$ in het lichaam te kunnen beschrijven hebben we het verloop in de verdeling van de radioactiviteit in het bloed en de organen van patiënten gemeten. Naast de radioactiviteit is 
ook de concentratie van het eiwit annexine $\mathrm{A} 5$ in bloed gemeten. Daarbij zijn de proefpersonen tot minimal 4 uur na het inspuiten van het annexine A5 gevolgd. De eerste methode (het z.g. Imino-annexine A5) om annexine A5 radioactief te maken leverde een onvoldoende stevige binding van de radioactiviteit aan het annexine A5 op. Hierop werd besloten om voor de tweede onderzochte methode om annexine A5 radioactief te maken (het BTAP-annexine A5), de stabiliteir van her madioactieve annexine A5 te onderzoeken. Verder zijn de annexine A5 concentraties gemeten in een aparte groep met patiënten die net een hartinfarct hadden doorgemaakt. Deze gegevens zijn vergeleken met de patiëntengroep die in dit onderzoek werden behandeld met radioactief annexine $\mathrm{A} 5$. Voor het meten van de verdeling van de radioactiviteit in de organen is gebruik gemaakt van opnamen met een gammacamera. We hebben de verkregen gegevens geanalyseerd met verschillende computermodellen om de verdeling van radioactief annexine $A 5$ in de mens te beschrijven.

De verdeling van radioactief annexine $A 5$ in het bloed kon beschreven worden met een model, waarbij voor beide types annexine A5 de verdwijning uit het bloed plaatsvindt met een verloop in twee fasen. De eerste fase heeft een halveringstijd van de concentratie in het bloed van ongeveer 15 minuten, terwijl de tweede fase een halveringstijd heeft van ca, 4 uur. Het verschil tussen Imino-annexine A5 en BTAP-annexine A5 bestaat vooral hieruit, dat de aan BTAP-annexine A5 gebonden radioactiviteit veel sneller door het lichaam uitgescheiden wordt dan bij Iminoannexine A5 het geval is. Hoewel de halveringstijden ongeveer gelijk zijn, is de hoeveelheid radioactiviteit die bij de verdeling in het bloed voor BTAP-annexine A5 wordt omgezet groter dan voor Imino-annexine A5.

We hebben kunnen aantonen, dat BTAP-annexine A5 stabiel is in her bloed van patiënten tot 20 uur na injectie. De gemeten annexine $A 5$ concentraties in her bloed van patiënten vertoonden veel variatie. De gevonden waarden $k$ wamen ongeveer overeen met die van de controlegroep van patiënten die net een hartinfarct hadden doorgemaakt. We weten niet goed wat de oorzaak van deze schommelingen is. Het gevolg is wel, dat het hierdoor moeilijk is om een goede beschrijving te geven van de verdeling van het annexine $\mathrm{A} 5$ zelf in her lichaam, in tegenstelling tot de verdelling van de radioactiviteit. Verder konden we aantonen dat de radioactiviteit voor zowel Imino-annexine A5 als BTAP-annexine A5 zich sterk ophoopt in lever en nieren. Dit laatste heeft niets te maken met celdood in deze organen, maar is waarschijnlijk het gevolg van de stof wisseling die annexine $\mathrm{A} 5$ zelf of de an annexine $\mathrm{A} 5$ gebonden radioacriviteit ondergaar. BTAP-annexine $A 5$ wordt bovendien heel snel in de darmen zichtbaar. Met beide types annexine A5 was het mogelijk celdood op te sporen in patiënten die net een hartinfarct hebben doorgemaakt, in patiënten met vormen van kanker in hoofd en nek, in de ledematen en bij borstkanker. Door de grote hoeveelheid tadioactiviteit die we in lever, nieren en darmen hebben aangetroffen, is het niet mogelijk celdood in deze organen op te sporen. Daarom is het nodig verder te zoeken naar nieuwe methoden om annexine $\mathrm{A} 5$ radioactief te maken of zelfs naar 
heel nieuwe methoden om celdood zichtbaar te maken.

In Hoofdstuk 4 hebben we alleen het BTAP-annexine A5 onderzocht. Opnieuw is de verdeling van de stof in het lichaam onderzocht, maar op een andere manier. De opname van annexine A5 in de afzonderlijke organen is gemeten en verder is ook de stralingsbelasting per orgaan (bijvoorbeeld lever, nieren, darmen) gemeten in de tijd. Daarbij hebben we de in hoofdstuk 3 gevonden uitkomst dat de opname wan de radioactiviteit in lever en nieren erg hoog is nog beter kunnen bewijzen. Hiermee is bijwoorbeeld aangetoond, dat de opname van radioactiviteit in lever en nieren vrij lang duurt (ongeveer 16 uur), en dat hierdoor de belasting met radioactiviteit nog verder toeneemt. Opvallend is, dat de opname van radioactiviteit op de platsen waar celdood gemeten werd heel laag is. Er zijn dus relatief weinig annexine A5 deeltjes nodig on celdood zichtbaar te kunnen maken. Verder hebben we door dit onderzoek een inschatting kunnen maken van het uitscheidingspatroon van de tadioactiviteit.

Ongeveer $75 \%$ van de annexine A5 radioactiviteit wordt via de urine uitgescheiden, terwijl ca. $25 \%$ via de ontlasting het licham verlaat. Hoewel de verblijfstijd wan de radioactiviteit relatief lang is, is de uiteindelijke stralingsbelasting ongeveer net zo groot als voor andere radioactieve stoffen die we dagelijks in onderzoek met patiënten gebruiken.

Tenslotte hebben we een onderzoek gedaan waarbij gekeken is of een behandelingsmethode die celdood remt gemeten kan worden met zowel radioactief als Auorescent gemaakt annexine A5.

In Hoofdstuk 5 hebben we de celdoodremmende eigenschappen van minocycline onderzocht. Dit is een veelgebruikt antibioticum waarvan eigenlijk pas de laatste jaren bekend is, dat het celdood kan remmen. Om het effect van minocycline te meten hebben we twee proefdierstudies uitgevoerd. In beide studies hebben we bij muizen en konijnen een afsluiting van de kransslagader aangebracht, waa rdoor een situatie wordt nagebootst die op een hartinfarct lijkt. Door de afsluiting na 30 of 40 minuten weer op te heffen wordt het openmaken van her bloedvat geimiteerd, zoals dit bij patiènten met een hartinfarct valk wordt uitgevoerd. De celdood die tijdens dit proces optreedt, wordt veroorzaakt door zuurstofgebrek in de hartspietcellen, waarna bij opheffing van de afsluiting juist zuurstofdeeltjes en andere reactieve stoffen ontstaan die de cel zichzelf laten beschadigen. In de eerste studie hebben we bij muizen een afsluiting van de kransslagader veroorzaakt. Na 30 minuten is de afsluiting opgeheven. Vervolgens is eerst met een injectie minocycline aan de muizen gegeven en daarna Huorescent annexine A5. De controlegroep ontving geen minocycline, maar een injectie met een zoutoplossing. Daana is onder een fluorescentiemicroscoop de ophoping van fluorescent annexine A5 in her hart van de muizen bekeken. Hiervan zijn digitale foto's genomen ${ }_{\Perp}$ die met een computerprogramma geanalyseerd zijn.

Op een vergelijkbare manier hebben we het afsluiten en openen van een kransslagader bij konijnen onderzocht. Hierbij is radioactief annexine A5 ingespoten. Kort voot het doodmaken van de konijnen is de kransslagader nog eens dichtgemaakt en een andere 
radioacrieve stof, ${ }^{201} \mathrm{Tl}$, bij de konijnen ingespoten om de doorbloeding van het hart te meten. Dit laatste is gedaan om de grootte van het infarctgebied te kunnen bepalen. We hebben her hart uit ieder konijn genomen om de gebieden waarin het hartinfarct is opgetreden zichtbaar te maken met een gammacamera. Verder is de radioactiviteit in de konijnenharten nauwkeurig gemeten, zodat van het gehele hatt een kaart gemaakt kon worden met daarop de verdeling van radioactiviteit. De radioactiviteitsverdeling in het hart van de konijnen heeft een directe relatie met de hoeveelheid celdood die in de verschillende gebieden van het hart is opgetreden. Voor de experimenten in muizen en konijnen is de helft van de dieren ( 3 stuks) mer minocycline behandeld. We hebben bij beide onderzoeken kunnen aantonen, dat de opname van fuorescent of radioactief annexine A5 lager is na toediening van minocycline aan de dieren. Dit betekent dat minocycline een remmend effect heeft op het optreden van celdood in een op een hartinfarct lijkende situatie bij proefdieren. Minocycline zou bij patiënten met een hartinfarct een beschermend effect kunnen hebben. Dit is een belangrijk onderwerp voor onderzoek in de toekomst. 


\section{Conclusies}

Door het uitwoeren van het in dit proefschrift beschreven onderzoek is meer informatie verzameld over de mogelijkheden om met annexine A5 celdood op te sporen. We hebben een methode ontwikkeld om het PS-bindend vermogen van radioactief of fluorescent annexine A5 te kunnen vaststellen. Hierdoor is een snelle kwaliteitscontrole mogelijk voordat annexine A5 bij een patiënt geïnjecteerd wordt. Verder is de verdeling van radioacrief annexine $A 5$ in patiënten onderzocht. Dit heeft informatie opgeleverd over de hoge stapeling van radioactiviteit in met name lever en nieren. De problemen die dit oplevert bij het maken van opnamen in patiënten kunnen we in de toekomst gebruiken om betere manieren te ontwikkelen om annexine A5 radioactief te maken. Tenslotte hebben we kunnen aantonen, dat annexine A5 geschikt gemaakt kan worden om te gebruiken als middel bij het bepalen van de effectiviteit van een behandeling. We hebben dit getest in proefdierexperimenten waarbij een hartinfarct werd nagebootst. In met minocycline behandelde dieren werd remming van celdood aangetoond. Overigens is een vergelijkbare methode al gebruikt om het effect van bestraling bij patiënten met kanker te kunnen vaststellen. Het uitvoeren van meer onderzoek met annexine A5 in pariënten met bijvoorbeeld kanker of harten vaatziekten is erg belangrijk. Hiermee kunnen we wellicht aantonen dat annexine A5 gebruikt kan worden als standaard methode om het effect op celdood te kunnen bepalen. 


\subsection{Dankwoord/ Acknowledgments}

Het in dit proefschrift beschreven onderzoek is allerminst een eenmansprestatie. Natuurlijk heb ik uiteindelijk zelf de resultaten verzameld en het meeste schrijfwerk verricht. Maar bijhet verkrijgen van de hiergepresenteerde resultaten is sprakegeweest van uitgebreid teamwerk. Er zijn zelfs meerdere teams bij betrokken geweest!

Allereerst het Annexineteam, want zo kunnen we dit netwerk van zeer gedreven wetenschappers wel noemen. Rond Chris Reutelingsperger (co-promotor) en Leo Hofstra (had ook co-promotor kunnen zijn) is een zeer divers gezelschap actief van biochemici, medische promovendi, nucleair geneeskundigen en analisten. Gehypnotiseerd door Chris's zeer aanstekelijke verhalen over annexine A5 en Leo Hof stra's creatieve geest die overal wel een nieuw soott onderzoek lijkt te vinden, raakt menig ( $\mathrm{PhD}$-)student hopeloos verstrikt in het annexine-netwerk. En met succes! Ewald Dumont, Abdelkader Bennaghmouch, Heidi Kenis, Hugo van Genderen, Bas Kietselaer (paranimf), Johan Verjans, Reinier Zandbergen, Maarten Corsten en Sander Wolters zijn voorlopig aan annexine A5 vastgeklonken, en ikzelf ook.

Alle mensen binmen het Annexineteam ben ik heel dankbaar voor de door hun geboden teamgeest en hulp bij het verwezenlijken van dit proefschrift.

Zonder de afdeling Nucleaire Geneeskunde in het azM en haar inbreng binnen het Annexineteam had mijn promotieonderzoek niet kunnen plaatsvinden. Veel dank ben ik verschuldigd aan mijn promotor Prof. dr. Guido Heidendal. Je hebt samen met Leo en Chris de basis gelegd voor het eerste onderzoek met radioactief annexine A5 in patiënten. Ik denk dat we daar heel erg trots op mogen zijn. Je bent voor mij een heel plezierige promotor geweest. Je wist een goede balans te vinden tussen het geven van ruimte enerzijds en tegelijk probeerde je anderzijds op jouw wijze sturing te geven aan het onderzoek. Bovendien ben je iets begonnen waar we mee verder kunnen en willen. Nu Prof. dr. Jaap Teule hoofd van de afdeling Nucleaire Geneeskunde is, zijn er wel andere wegen ingeslagen, mar we doen nog steeds onderzoek met annexine A5.

Over Chris Reutelingsperger (co-promotor) kan ik heel kort zijn. $\mathrm{Na}$ al onze gesprekken over dat ene citaat van George B. Shaw ("Ik heb je een lange brief gestuurd omdat ik geen tijd had voor een korte.") weet ik nu ongeveer hoe ik voor een Engelstalig artikel allerlei zinsconstructies in elkaar moet passen en hiervan een farsoenlijk geheel moet maken. Ik heb veel var je geleerd: op onderzoeksgebied nog meer dan qua taalkundig inzicht. Veel dank voor alle tijd die je had om me op weg te helpen met dit proefschrift.

Als dit proefschrift een gerecht is, dan is collega ziekenhuisapotheker Leo Stolk (copromotor) de kok geweest die klaarstond met de farmaceutische kruiden. Je hebt het onderzoek in dit proefschriftgetoetst aanje farmaceutische smaak. Mijn dank hiervoor is groot. Je inzichten op het gebied van de klinische farmacologie en de farmaceurische analyse hebben een belangrijke bijdrage geleverd aan het manuscript. 
Mijn opleider Ton Beysens heb ik heel veel dank verschuldigd voor het bieden van de mogelijkheid en de daarbij behorende tijd om aan dit proefschrift te kunnen werken. Je hebt tijdens mijn opleidingstijd samen met Guido Heidendal richting gegeven aan radioactief annexine $\mathrm{A} 5$ als onderzoeksonderwerp voor de registratielezing, en in de tijd daarna hebben we dit kunnen uitbouwen tot een volwaardig proefschrift. Hoewel je de laatste twee jaar minder betrokken was bij het onderzoek op zich, kijk ik zeker terug op een plezierige samenwerking.

Dr. Gerrit Kemerink was vooral in de eerste jaren een zeer trouw lid van het Annexineteam. Jouw artikelen over de biodistributie en dosimetrie van radioactief annexine A5 in proefpersonen zijn onovertroffen. Ik ben je veel dank schuldig voor alle geboden hulp bij hoofdstuk 3 en 4 van het proefschrift. Ik voorzie dat we in het kader van allerlei PET-projecten de komende tijd nog veel met elkaar te maken krijgen, en ook dat lijkt me een heel goed vooruitzicht.

Leo Hofstra is een belangrijke factor geweest bij het totstandkomen van het proefschrift. Je bent niet alleen enorm creatief als onderzoeker, maar ook, samen met Chris Reutelingsperger, in het aanboren van allerlei financiële bronnen. Ik ben in de loop van de jaren een dankbare gebruiker van beide eigenschappen geweest en hoop in de toekomst het nodige terug te kunnen doen.

De arts-assistenten Nucleaire Geneeskunde Ing Han Liem en Willy Thimister hebben vooral gedurende de beginperiode een grote bijdrage geleverd aan het klinische werk, dat in hoofdstuk 3 en 4 beschreven is. Ik heb hiervoor veel waardering en vond het ook leuk met jullie samen te werken. Waander van Heerde bedank ik voor de vele discussies over annexine A5 kinetiek en -bloedspiegels. Dankzij Peter Moonen en de met hem gevoerde discussies over hoofdstuk 2 is het daarin gepresenteerde onderzoek nog scherper geformuleerd.

Bij het onderzoek waren diverse verpleegafdelingen en vakgroepen betrokken. Hen bedank ik voor alle hulp, en de patiënten en proefpersonen voor het deelnemen aan de kinetiek-en biodistributiestudies.

Prof Dr. Jagat Narula and his staff at the University of California in Irvine are greatly acknowledged for their contribution to the chapters 1 and 5 of this dissertation. Dear Jagat, your inspiring view on clinically oriented research stimulated me very much. Thanks also for being a member of the Thesis-evaluation committee. Many thanks are expressed to Dr. Artiom Petrov, Dr. Masayoshi Sarai, and Dr. Satoshi Isobe for doing many experiments within a narrow time frame. All other members at the Department of Cardiology were great hosts. It was a privilege to work with you.

De beoordelingscommissie bestaande uit Prof. dr. G.J.J. Teule, Mw. Prof. dr. C. Bruggeman, Prof. dr. J. Narula, Dr. C. Neef en Prof. dr. A. Verbruggen dank ik hartelijk voor het beoordelen van het manuscript.

Zeer belangrijke ondersteunende mensen bij het uitvoeren van de experimenten waren te vinden binnen de afdeling Nucleaire Geneeskunde, en de afdelingen Biochemie en Cardiologie van de UM. Marie-Thérèse Pakbiers (paranimf) en Denise Janssen 
hebben zich in de eerste tijd dar het annexine-onderzoek van start ging opgeworpen als onze "hoofdlabelaars", die ook op minder gunstige tijdstippen klaarstonden als er weer eens cen patiënt met acuut myocardinfarct was aangemeld. Natuurlijk hebben ook de andere medewerkers van de afdeling zich onder leiding van Servé Halders ingezet om dit proefschrift te kunnen laten slagen. Mijn dank hiervoor is groot.

Nicole Bitsch heeft een grote rol gespeeld bij het uitvoeren van het meeste proefdieronderzoek met muizen. Dank zij jouw waardige handen ligt er nu een boekje. Ik vond het heel leuk met je samen te werken. Eigenlijk moet ik ook de proefdieren bedanken, want zonder hen was hoofdstuk 5 nier geschreven. Ik dank in samenhang met al het proefdierwerk de collega's van de afdeling Farmacologie voor het mogen gebruiken (onveilig maken?) van hun gang en werkruimten.

Niko Deckers heeft veel hulp geboden bij het uitvoeren van allerlei experimenten, met name voor het onderzoek beschreven in hoofdstuk 2. Ook als er op een onverwacht tijdstip weer eens iets geregeld moest worden, stond je voor me klaar. Op vergelijkbare wijze heeft Perra Lux de nodige annexine ELISA's gedaan voor het onderzoek in hoofdstuk 3. De andere mensen bij Biochemie dank ik voor de gezellige sfeer op het lab.

Een onmisbare inbreng bij het kunnen schrijven van dit proefschrift is de bijdrage van mijn collega ziekenhuisapothekers (al dan niet in opleiding) en andere medewerkers van de apotheek. Zonder alle compensatie vanuit dit Apotheekteam was dit proefschrift niet tot stand gekomen. En het was bepaald niet alleen wetenschappelijk onderzoek dat ons bezighield! We zijn ook op andere fronten (reorganisatie, elektronisch voorschrijven, nieuw computersysteem) nogal druk geweest.

Ik bedank Eugène Hardy voor alle dagen dat hij de administratie van de cytostaticabereidingen van mij heeft overgenomen, zodat ik weer wat experimenten kon uitvoeren. Verder ben ik Nicole Veldhorst, en ook Hein van Onzenoort en Afke van der Plas zeer dankbaar voor alle hulp bij het oppakken van mijn nieuwe taak als logistieke apotheker. Hugo van der Kuy heeft over een groot aantal zaken in relatie met mijn werkzaamheden in de afgelopen jaren meegedacht, veel dank daarvoor. Met Jo Vankan en Sam Stijns hoop ik snel de bedrijfswoering in het magazijn/ logistiek van de apotheek in orde te krijgen. Veel dank voor jullie begrip en geduld bij het totstandkomen van dit proefschrift. Kees Neef bedank ik voor het feit dat hij het nieuwe hoofd van de apotheek is geworden. Ik spreek de hoop uit, dat we samen de aporheek nog beter kunnen maken dan hij al is.

Her Familieteam heeft in de afgelopen jaren veel bijgedragen aan de randvoorwaarden die nodig zijn voor her schrijven van bijvoorbeeld een proefschrift. Mijn ouders ben ik heel veel dank verschuldigd. Jullie steun bij al mijn genoten onderwijs \& opvoeding was al geweldig. Deze keer dank ik jullie voor alle belangstelling, oppasdagen, tuin-en andere praktische klussen. En bovendien ook nog voor de bijdrage aan de Nederlandstalige samenvatring van her proefschrift. Ner zo onmisbaar zijn mijn schoonouders. Het gemak warmee jullie vieren de afstand Friesland c.q. Groningen- 
Limburg overbruggen spreekt boekdelen.

Verdere familie (met name Roel, Bas, Paulien en Oma Rigtje) dank ik hartelijk voor hun warme belangstelling en steun. We hebben de afgelopen tijd elkaar misschien niet vaak gezien, maar we wisten elkaar wel te vinden wanneer dat nodig was.

Onze vrienden dank ik voot hun vriendschap en belangstelling. Ik zie er nu al natr uit om ook met jullie het promotiefeest te kunnen vieren.

Het Thuisteam heeft een bijzonder grote inbreng gehad in het laten slagen van de missie die promoveren heet. Waar ben ik zonder Anoesjka? We zijn nu ongeveer twaalf jaar samen, maar volgens mij heb ik nog steeds niet helemaal door hoe goed het leven met jou is. Dank voor alles. Ook voor je soms zeer kritische inbreng bij alle hier beschreven experimenten.

Ben en Minke zijn gewoon de leukste kinderen die we ons hadden kunnen wensen.

Hendrikus Boersma

Januari 2006 


\subsection{Curriculum Vitae}

Hendrikus Boersma werd op 28 juni 1968 geboren in Leeuwarden. Hij behaalde aan het Lienward College in deze plaats zowel het HAVO als het VWO diploma. In 1988 ging hij Farmacie studeren aan de Rijksuniversiteit Groningen. Tijdens zijn afstudeerproject binnen de specialisatie fytofarmacologie werd de interactie tussen de cytostatische effecten van 5-fluorouracil en quercetine onderzocht. In 1994 werd het doctoraal examen Farmacie afgelegd, waarna in 1996 het apothekersdiploma werd behaald.

Vervolgens werden respectievelijk functies als kwaliteitsfunctionaris (Tramarko B.V. Almere) en projectapotheker (Slotervaart ziekenhuis, Amsterdam) vervuld.

In januari 1998 werd gestart met de opleiding tot ziekenhuisapotheker in het academisch ziekenhuis Maastricht (opleider: drs. A.J.M.M. Beijsens). Het bijbehorende registratieonderzoek was getiteld ${ }^{49 m} \mathrm{~T} c$-Annexine $V$ in patiënten meteen hartziekte. Detectie van apoptose door SPECT-beeldvorming. Dit onderzoeksproject vormde de aanzet voor het hier voltooide proefschrift. De inschrijving in het specialistenregister voor ziekenhuisapothekers vond plaats per november 2000 . In de periode 2000-2004 heeft hij zich als ziekenhuisapotheker in het azM met name bezig gehouden met de bereiding van injectie-en infuuspreparaten (o.a. cytostatica), en arbeidsomstandigheden $\mathrm{m}$.b.t. geneesmiddelenverwerking.

Hendrikus Boersma volgde van 2001-2003 de opleiding tot klinisch farmacoloog, waarvoor de bijbehorende antekening op 11 april 2003 werd verkregen (opleider: dr. L.M.L. Stolk).

Sinds maart 2005 is hij farmaceutisch eindverantwoordelijk voor de sectie Distributie en Opslag binnen de afdeling Klinische Farmacie. Tevens is hij als toezichthouder verantwoordelijk voor de bereiding van radiofarmaca binnen de afdeling Nucleaire Geneeskunde.

Van 1999-2005 is voor dit proefschrift onderzoek verricht in samenwerking met de afdelingen Nucleaire Geneeskunde en Cardiologie van het azM, de afdeling Biochemie van de Universiteit Maastricht, en de afdeling Cardiologie van de Universiteit van Californië in Irvine (USA). Begeleiders waren Prof, dr. G.A.K. Heidendal, dr. C.P.M. Reutelingsperger, dr. L. Hofstra, dr. L.M.L. Stolk en Prof. dr. J. Narula. Hendrikus is gehuwd met Anoesjka van den Broek. Zij hebben een zoon, Ben en een dochter, Minke. 


\subsection{About the author}

Hendrikus H. Boersma was born in Leeuwarden, the Nerherlands on June 28 , 1968. He obtained his secondary education degree at Lienward College in the same town in 1988. In the same year, he started his Pharmacy studies at Groningen State University in Groningen, the Netherlands. During this education, he specialized in phytopharmacology. His PharmD-dissertation was entitled 'Interaction berween the cyrostatic effects of quercetin and 5-Auorouracil in two human colorectal cancer cell lines'.

He obtained his PharmD degree in 1996. Subsequently, he started to work as a quality assurance officer (Tramarko BV, Almere, the Netherlands), followed by a position as pharmacist at the Slotervaart Hospital in Amsterdam. In 1998, he started his training in Clinical Pharmacy at the Maastricht University Hospital in Maastricht, the Netherlands (Supervisor: A.J.M.M. Beysens). During this training he started to study radiolabeled annexin $\mathrm{A} 5$, which led to the $\mathrm{PhD}$-thesis presented here. He was registered as a Clinical Pharmacist in 2000.

From 2000 up until now he has been working in the Maastrichr University hospital as a staff pharmacist. From 2000-2004 he was responsible for the preparation of e.g. cytostaric drugs. As well, he managed several projects concerning exposure hazard of drugs.

From 2001-2003, Hendrikus was a trainee in Clinical Pharmacology (Supervisor: Dr. L.M.L. Stolk). He was certifed as a Clinical Pharmacologist in the same year. From March 2005, he has been responsible pharmacist for the division of Medication Logistics and Clinical trials. Furthermore, he is radiopharmacist at the Department of Nuclear Medicine.

From 1999-2005, he was performing the research which is presented in this thesis. These research projects were done in collaboration with the Departments of Nuclear: Medicine and Cardiology at the Maastricht University Hospital, the Department of Biochemistry at Maastricht University, and the Department of Cardiology at the University of Califonia, Irwine, Irvine, USA. Supervisors of his PhD-thesis were: Prof. G.A.K. Heidendal, Dr. C.P.M. Reutelingsperger, Dr. L. Hofstra, Dr. L.M.L. Stolk, and Prof. J. Narula.

Hendrikus is married to Anoesjka van den Broek. They have a son, Ben, and a daughter, Minke. 